\title{
Article
}

\section{Safety and efficacy of fluoxetine on functional outcome after acute stroke (AFFINITY): a randomised, double-blind, placebo-controlled trial}

Hankey, Graeme J., Hackett, Maree, Almeida, Osvaldo P., Flicker, Leon, Mead, Gillian E., Dennisq, Martin S., Etherton-Beer, Christopher, Ford, Andrew H., Billot, Laurent and Et, Al

Available at http://clok.uclan.ac.uk/35750/

Hankey, Graeme J., Hackett, Maree ORCID: 0000-0003-1211-9087, Almeida, Osvaldo P., Flicker, Leon, Mead, Gillian E., Dennisq, Martin S., Etherton-Beer, Christopher, Ford, Andrew H., Billot, Laurent et al (2020) Safety and efficacy of fluoxetine on functional outcome after acute stroke (AFFINITY): a randomised, double-blind, placebo-controlled trial. The Lancet Neurology, 19 (8). pp. 651-660. ISSN 1474-4422

It is advisable to refer to the publisher's version if you intend to cite from the work. http://dx.doi.org/10.1016/S1474-4422(20)30207-6

For more information about UCLan's research in this area go to http://www.uclan.ac.uk/researchgroups/ and search for <name of research Group>.

For information about Research generally at UCLan please go to http://www.uclan.ac.uk/research/

All outputs in CLoK are protected by Intellectual Property Rights law, including Copyright law. Copyright, IPR and Moral Rights for the works on this site are retained by the individual authors and/or other copyright owners. Terms and conditions for use of this material are defined in the policies page. 


\section{The Lancet Neurology}

\section{Safety and efficacy of fluoxetine on functional outcome after acute stroke (AFFINITY): a randomised, double-blind, placebo-controlled trial.

\author{
--Manuscript Draft--
}

\begin{tabular}{|c|c|}
\hline Manuscript Number: & THELANCETNEUROLOGY-D-20-00293R2 \\
\hline Article Type: & Article (Clinical Trials) \\
\hline Keywords: & Stroke; fluoxetine; SSRI; randomised trial; recovery; functional outcome \\
\hline Corresponding Author: & $\begin{array}{l}\text { Graeme J Hankey } \\
\text { Perth, AUSTRALIA }\end{array}$ \\
\hline \multirow[t]{10}{*}{ Order of Authors: } & Graeme J Hankey \\
\hline & Maree L Hackett \\
\hline & Osvaldo P. Almeida \\
\hline & Christopher Etherton-Beer \\
\hline & Andrew $\mathrm{H}$. Ford \\
\hline & Laurent Billot \\
\hline & Stephen Jan \\
\hline & Erik Lundström \\
\hline & Craig S. Anderson \\
\hline & Huy Thang-Nguyen \\
\hline Abstract: & $\begin{array}{l}\text { Background } \\
\text { Trials of fluoxetine for recovery after stroke report conflicting results. The Assessment } \\
\text { oF FluoxetINe In sTroke recoverY (AFFINITY) trial aimed to determine if daily } \\
\text { fluoxetine for } 6 \text { months after stroke improves functional outcome in Australasian and } \\
\text { Vietnamese patients. } \\
\text { Methods } \\
\text { AFFINITY was a randomised, parallel-group, double-blind, placebo-controlled trial } \\
\text { conducted in } 43 \text { hospital stroke units in Australia (n=29), New Zealand (4), and } \\
\text { Vietnam (10). Eligible patients were adults with a clinical diagnosis of stroke in the } \\
\text { previous } 2-15 \text { days and a persisting neurological deficit. Patients were randomised via } \\
\text { a web-based system using a minimisation algorithm to once daily, oral fluoxetine } 20 \mathrm{mg} \\
\text { or matching placebo for } 6 \text { months. Patients, investigators and outcome assessors were } \\
\text { masked to the treatment allocation. The primary outcome was functional outcome, } \\
\text { measured by the modified Rankin scale (mRS), at } 6 \text { months. The primary analysis was } \\
\text { an ordinal logistic regression of the mRS at } 6 \text { months, adjusted for minimisation } \\
\text { variables. Analyses were according to the patient's treatment allocation. The trial is } \\
\text { registered with the ACTRN registry, number } 12611000774921 \text {. } \\
\text { Findings }\end{array}$ \\
\hline
\end{tabular}


1280 patients were recruited in Australia $(n=532)$, New Zealand $(n=42)$ and Vietnam $(n=706)$ between 11 January 2013 and 30 June 2019; 642 were allocated fluoxetine and 638 placebo. Adherence to trial medication (mean 167 [SD 48] days) was similar between groups. At 6 months, mRS data were available in $624(97.2 \%)$ patients allocated fluoxetine and $632(99.1 \%)$ placebo. The distribution of $\mathrm{mRS}$ categories at 6 months was similar in the fluoxetine and placebo groups (adjusted common odds ratio 0.936, 95\% Cl 0.762-1.150; $p=0.53$ ), and consistent among all pre-defined subgroups. Compared to placebo, patients allocated fluoxetine had more falls (20 [3.12\%] vs 7 [1.10\%]; $p=0.02)$, bone fractures $(19$ [2.96\%] vs 6 [0.94\%]; $p=0.01)$ and epileptic seizures $(10[1.56 \%]$ vs $2[0.31 \%] ; p=0.04)$ at 6 months.

Interpretation

Fluoxetine 20mg daily for 6 months after acute stroke did not improve functional outcome and increased the risk of falls, bone fractures, and seizures. These results do not support the use of fluoxetine to improve outcome after stroke.

Funding

National Health and Medical Research Council of Australia (Project Grant 1059094). 
May 1, 2020

Dr. Melanie Wulff

Senior Editor, The Lancet Neurology,

The Lancet, Hackerbrücke 6, Munich 80335, GERMANY

m.wulff@elsevier.com

Manuscript reference number: THELANCETNEUROLOGY-D-20-00293R1

Title: Effects of fluoxetine on functional recovery after acute stroke (AFFINITY): a randomised, double-blind, placebo-controlled trial.

Dear Melanie,

Thank you for submitting your email dated April 27, 2020 requesting a response to the reviewers' and editorial comments.

Editorial points to be addressed:

- Study title: To fit Lancet style, l'd suggest the following title "Safety and efficacy of fluoxetine on functional recovery after acute stroke (AFFINITY): a randomised, double-blind, placebo-controlled trial." What do you think?

The title has been changed as proposed to "Safety and efficacy of fluoxetine on functional outcome after acute stroke (AFFINITY): a randomised, double-blind, placebo-controlled trial"

The only change to your suggestion is that we have accepted the recommendation of the reviewer \#3 to replace "recovery" with "outcome" as much as possible. If you disagree, and feel we should keep recovery in the title, that is also fine with us.

- Could we cut down a couple of words here and there so have max $\mathbf{4 6 0 0}$ words (our limit is normally $\mathbf{4 5 0 0}$ words).

The word count has been reduced to 4597 words

- Appendix: for the Kaplan Meier survival curve- Please add $95 \% \mathrm{Cl}$ to all time-to-event data and other data derived from Kaplan-Meier analyses and please include number at risk (and, if available, number censored) in each group for each time.

The Supplementary figure 3, Kaplan Meier curves has been modified with $95 \% \mathrm{Cl}$ of time to event data, and the number at risk in each group for each time.

- Our journal office is currently checking if we have all forms (ie, signatures and ICMJE forms for the writing group members). 
To enable readers to better appreciate research findings and to encourage full and transparent reporting of outcomes, The Lancet family journals offer to publish a web address in accepted paper that links to the study's protocol on the author's institutional website (see Lancet 2009; 373: 992). This is particularly encouraged for randomised controlled trials, but is welcome for all types of research.

Thank you

Public access to the AFFINITY trial full protocol is available from the AFFFINITY trial website:

https://www.affinitytrial.org/

(accessed April 30, 2020)

The direct web address for the protocol is:

https://www.affinitytrial.org/download/researcher\%27s documents/Case Report Forms v2/Affinit y-Protocol-Version-5-clean-copy-18112015.pdf

(accessed April 30, 2020)

When you submit the revised paper, please provide one "clean" copy and one copy where your changes are highlighted. In addition, please provide a separate document listing the editorial and referee comments and your replies, point by point. These files must be submitted as MS Word files.

One clean copy and one copy with highlighted changes are submitted as MS word files

Reviewers' comments:

Reviewer \#3: Discussion: The additional information regarding the FLAME trial is useful, though reporting of the baseline data to demonstrate imbalance is not needed, given that the effects of fluoxetine remained significant when analyses were adjusted for baseline values. Instead, the authors could add data demonstrating the difference in baseline severity between FLAME and AFFINITY. This is more relevant.

The unbalanced baseline data have been deleted, but the difference in baseline severity between FLAME and AFFINITY has been added, as stated:

On page 16 , para 1 , it now reads

"Patients enrolled in FLAME had more severe strokes (mean baseline NIHSS 13 vs median NIHSS 6 in AFFINITY)."

Thank you for pointing out that the percentage of women in the sample is almost identical to FOCUS. Please add this to the manuscript. 
It is now stated, page 15, para 2:

"The AFFINITY trial was smaller than the FOCUS trial9 but both trials recruited patients of similar sex (women 38\%), stroke severity (median NIHSS=6) and at a similar time (one week, mean) after stroke onset."

Methods, Study design and participants, second paragraph, second sentence: "Patients were excluded if there was any definite indication for fluoxetine (e.g. depression)...". This creates uncertainly because 32 patients with depression were recruited (Table 1). It seems possible that fluoxetine may have been indicated but was not taken. This was raised in previous comments and remains unclear.

Patients could have depression but not require treatment with an SSRI such as fluoxetine (e.g. nonpharmacological treatment with clinical psychology, or pharmacological treatment with a non-SSRI.), and therefore be eligible for inclusion in the trial.

We have removed "(e.g. depression)" to avoid confusion, and because fluoxetine could be definitely indicated for other conditions (e.g. anxiety).

Please be more precise in your use of the term "recovery", throughout the manuscript. The mRS at 6 months is a measure of outcome, not recovery. Recovery is improvement over time, so measuring recovery requires at least two measures to be made at two time points, to quantify change over time (as per FLAME, for example). A single measure at the end of the trial reflects the outcome, not recovery. Therefore, most instances of "recovery" in the manuscript need to be replaced with "outcome".

Most instances of "recovery" in the manuscript have been replaced with "outcome".

\section{Reviewer \#4: MINOR COMMENSTS}

I have only $\mathbf{2}$ minor issues to consider for revision:

1. Abstract: "mood and emotional control" at 6 months are presented as a major finding in their first paragraph of their discussion, but no where else:

I quote: "Other major findings were that fluoxetine improved mood and emotional control but increased falls, fractures, and epileptic seizures at 6 months. Could we rephrase in the discussion?

"improved mood and emotional control" has been removed from the first paragraph in the discussion

The opening paragraph of the discussion now just reads:

"The main finding of the AFFINITY trial was that adding fluoxetine 20mg daily for 6 months after acute stroke to interdisciplinary stroke unit care did not improve functional outcome at 6 months in an ethnically diverse population. Other major findings were that fluoxetine increased falls, fractures, and epileptic seizures at 6 months." 


\section{Discussion, first paragraph of page 14: The lack of significant interaction effects of fluoxetine with motor recovery in the AFFINITY trial may also due to lower dosing of therapy in the first weeks post stroke when compared to the FLAME trial. However, this argument is not mentioned in the discussion. I suggest to consider this hypothetical factor as well in the text.}

The FLAME trial does not report a significant interaction between dose of fluoxetine (20 vs $40 \mathrm{mg}$ daily) and outcome.

The FLAME Trial manuscript states:

1. Use of functional MRI in other studies showed that single doses of fluoxetine and paroxetine overactivated motor cortices compared with placebo in both healthy individuals and patients with stroke

2. In Table 1, the only reported prospective randomised placebo-controlled clinical trials of selective serotonin-reuptake inhibitors in motor recovery after ischaemic stroke with fluoxetine was Pariente et al13 Fluoxetine $20 \mathrm{mg}$ (single dose).

3. Patients were randomly allocated to fluoxetine ( $20 \mathrm{mg}$ once per day, orally) or placebo for 90 days.

4. When depression occurred (according to clinical assessment) during the 3 months of treatment, clinicians were instructed to continue the study treatment (fluoxetine or placebo), to avoid use of any other antidepressant drugs and, if necessary, to give open-label fluoxetine (20 mg once a day) so that the patient received either $20 \mathrm{mg}$ (placebo group) or 40 mg per day (fluoxetine group). If a patient was given another antidepressant drug, the study treatment was stopped.

5. The frequency of depression was significantly higher in the placebo group (17 [29\%] patients) than in the fluoxetine group (four [7\%] patients; $p=0.002$ ).

So, only four patients assigned fluoxetine developed depression. It is not stated how many of them were treated with open label fluoxetine (to increase the daily dose to $40 \mathrm{mg}$ ) and when after randomisation, nor the effect of the higher dose on the primary outcome or mRS.

6. After adjustment of our analysis for clinical depression diagnosed before day 90, we noted that FMMS change between day 0 and day 90 was still significantly greater in the fluoxetine group (adjusted mean $34 \cdot 2$ points [95\% Cl 29.7-38.6]) than in the placebo group (24.2 [19.628.7]; $p=0.004)$.

a. i.e. after adjusting for those who may have had a higher dose of fluoxetine, there is no difference in the outcome, indeed the mean difference between groups is slightly higher at 10.0 (vs 9.7), suggesting that any higher dose did not have any incremental benefit.

We believe it is speculative, and probably incorrect, to suggest that there is a dose-response association between fluoxetine dose and outcome in FLAME.

So, we are not adding to this speculation in the discussion; we have already acknowledged that a higher dose of fluoxetine was not studied, in the limitations section of the discussion, page 14, as: 
"The dose of fluoxetine was $20 \mathrm{mg}$ once daily because this was the dose reported to be effective in the FLAME trial 5 and used in other fluoxetine trials for stroke recovery, 6 and is less likely to cause adverse effects than higher doses. However, we did not test higher doses of fluoxetine."

Reviewer \#5: Undoubtly, the paper has improved. The discussion is better and the way FLAME study is discussed much more appropriate. My only point is that in the abstract interpretation paragraph:

"Fluoxetine 20mg daily for 6 months after acute stroke did not improve functional recovery and increased the risk of falls, bone fractures, and seizures. These results do not support the use, or further trials, of fluoxetine to improve recovery after stroke."

the words "or further trials" should be supressed as there is no need to prevent people to do other clinical developments in severe stroke patients population which has not been addressed specifically.

As recommended, we have removed "further trials"; ... However, that was only included in the first revision as a response to comply with the recommendation of another reviewer.

Thank you for re-considering our revised version of the manuscript for publication in The Lancet Neurology.

Regards

Graeme Hankey, on behalf of the AFFINITY trial collaborative group. 
Items to include when reporting a randomized trial in a journal or conference abstract

\begin{tabular}{|c|c|c|}
\hline Item & Description & $\begin{array}{l}\text { Reported on } \\
\text { line number }\end{array}$ \\
\hline Title & Identification of the study as randomized & Line 2, page 1 \\
\hline Authors * & Contact details for the corresponding author & Bottom, page 1 \\
\hline Trial design & $\begin{array}{l}\text { Description of the trial design (e.g. parallel, cluster, non- } \\
\text { inferiority) }\end{array}$ & Line 6 , page 2 \\
\hline \multicolumn{3}{|l|}{ Methods } \\
\hline Participants & $\begin{array}{l}\text { Eligibility criteria for participants and the settings where } \\
\text { the data were collected }\end{array}$ & Lines 7-9 \\
\hline Interventions & Interventions intended for each group & 10 \\
\hline Objective & Specific objective or hypothesis & $3-4$ \\
\hline Outcome & Clearly defined primary outcome for this report & $11-12$ \\
\hline Randomization & How participants were allocated to interventions & 9 \\
\hline $\begin{array}{l}\text { Blinding } \\
\text { (masking) }\end{array}$ & $\begin{array}{l}\text { Whether or not participants, care givers, and those } \\
\text { assessing the outcomes were blinded to group } \\
\text { assignment }\end{array}$ & $10-11$ \\
\hline \multicolumn{3}{|l|}{ Results } \\
\hline $\begin{array}{l}\text { Numbers } \\
\text { randomized }\end{array}$ & Number of participants randomized to each group & 18 \\
\hline Recruitment & Trial status & $17-18$ \\
\hline $\begin{array}{l}\text { Numbers } \\
\text { analysed }\end{array}$ & Number of participants analysed in each group & $19-20$ \\
\hline Outcome & $\begin{array}{l}\text { For the primary outcome, a result for each group and the } \\
\text { estimated effect size and its precision }\end{array}$ & 22 \\
\hline Harms & Important adverse events or side effects & $23-25$ \\
\hline Conclusions & General interpretation of the results & $26-28$ \\
\hline Trial registration & Registration number and name of trial register & 15 \\
\hline Funding & Source of funding & 30 \\
\hline
\end{tabular}

${ }^{*}$ this item is specific to conference abstracts 


\section{CONSORT 2010 checklist of information to include when reporting a randomised trial*}

\begin{tabular}{|c|c|c|c|}
\hline Section/Topic & $\begin{array}{c}\text { Item } \\
\text { No }\end{array}$ & Checklist item & $\begin{array}{l}\text { Reported } \\
\text { on page No }\end{array}$ \\
\hline \multicolumn{4}{|l|}{ Title and abstract } \\
\hline & $1 \mathrm{a}$ & Identification as a randomised trial in the title & 1 \\
\hline & $1 \mathrm{~b}$ & Structured summary of trial design, methods, results, and conclusions (for specific guidance see CONSORT for abstracts) & 2 \\
\hline \multicolumn{4}{|l|}{ Introduction } \\
\hline Background and & $2 a$ & Scientific background and explanation of rationale & 5 \\
\hline objectives & $2 b$ & Specific objectives or hypotheses & 5 \\
\hline \multicolumn{4}{|l|}{ Methods } \\
\hline \multirow[t]{2}{*}{ Trial design } & $3 a$ & Description of trial design (such as parallel, factorial) including allocation ratio & 6 \\
\hline & $3 b$ & Important changes to methods after trial commencement (such as eligibility criteria), with reasons & $\mathrm{N} / \mathrm{A}$ \\
\hline \multirow[t]{2}{*}{ Participants } & $4 a$ & Eligibility criteria for participants & 6 \\
\hline & $4 b$ & Settings and locations where the data were collected & 6 \\
\hline Interventions & 5 & $\begin{array}{l}\text { The interventions for each group with sufficient details to allow replication, including how and when they were } \\
\text { actually administered }\end{array}$ & 7 \\
\hline \multirow[t]{2}{*}{ Outcomes } & $6 a$ & $\begin{array}{l}\text { Completely defined pre-specified primary and secondary outcome measures, including how and when they } \\
\text { were assessed }\end{array}$ & 9 \\
\hline & $6 b$ & Any changes to trial outcomes after the trial commenced, with reasons & $\mathrm{N} / \mathrm{A}$ \\
\hline \multirow[t]{2}{*}{ Sample size } & $7 a$ & How sample size was determined & 9,10 \\
\hline & $7 \mathrm{~b}$ & When applicable, explanation of any interim analyses and stopping guidelines & $\mathrm{N} / \mathrm{A}$ \\
\hline \multicolumn{4}{|c|}{ - } \\
\hline \multirow{2}{*}{$\begin{array}{l}\text { Sequence } \\
\quad \text { generation }\end{array}$} & $8 a$ & Method used to generate the random allocation sequence & 6 \\
\hline & $8 b$ & Type of randomisation; details of any restriction (such as blocking and block size) & 6 \\
\hline $\begin{array}{l}\text { Allocation } \\
\text { concealment } \\
\text { mechanism }\end{array}$ & 9 & $\begin{array}{l}\text { Mechanism used to implement the random allocation sequence (such as sequentially numbered containers), } \\
\text { describing any steps taken to conceal the sequence until interventions were assigned }\end{array}$ & 6 \\
\hline Implementation & 10 & $\begin{array}{l}\text { Who generated the random allocation sequence, who enrolled participants, and who assigned participants to } \\
\text { interventions }\end{array}$ & 6 \\
\hline Blinding & $11 \mathrm{a}$ & If done, who was blinded after assignment to interventions (for example, participants, care providers, those & 7 \\
\hline
\end{tabular}


assessing outcomes) and how

$11 \mathrm{~b}$ If relevant, description of the similarity of interventions

Statistical methods

$12 \mathrm{a}$

$12 b$

Statistical methods used to compare groups for primary and secondary outcomes

Methods for additional analyses, such as subgroup analyses and adjusted analyses

\section{Results}

Participant flow (a diagram is strongly recommended)

Recruitment

Baseline data

Numbers analysed

Outcomes and

estimation

Ancillary analyses

Harms

\section{Discussion}

\section{Limitations}

Generalisability

Interpretation

\section{Other information}

Registration

Protocol

Funding
$13 a$

For each group, the numbers of participants who were randomly assigned, received intended treatment, and were analysed for the primary outcome

$13 \mathrm{~b}$ For each group, losses and exclusions after randomisation, together with reasons

14a Dates defining the periods of recruitment and follow-up

14b Why the trial ended or was stopped

15 A table showing baseline demographic and clinical characteristics for each group

16 For each group, number of participants (denominator) included in each analysis and whether the analysis was by original assigned groups

17a For each primary and secondary outcome, results for each group, and the estimated effect size and its precision (such as 95\% confidence interval)

17b For binary outcomes, presentation of both absolute and relative effect sizes is recommended

18 Results of any other analyses performed, including subgroup analyses and adjusted analyses, distinguishing pre-specified from exploratory

19 All important harms or unintended effects in each group (for specific guidance see CONSORT for harms)

20 Trial limitations, addressing sources of potential bias, imprecision, and, if relevant, multiplicity of analyses

21 Generalisability (external validity, applicability) of the trial findings

22 Interpretation consistent with results, balancing benefits and harms, and considering other relevant evidence

23 Registration number and name of trial registry

24 Where the full trial protocol can be accessed, if available

25 Sources of funding and other support (such as supply of drugs), role of funders

\section{7}

10,11

11

12

12

11

12

1,2 tables

10,12

12,13

\section{3}

13

13

14,15

14

$15,16,17$

11

6 $3,11,26$

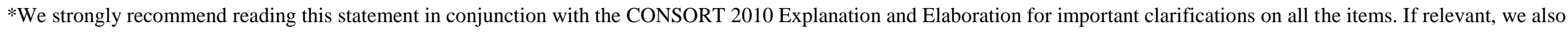

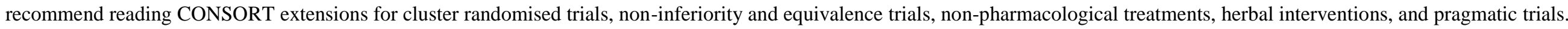
Additional extensions are forthcoming: for those and for up to date references relevant to this checklist, see www.consort-statement.org. 
Manuscript reference number: THELANCETNEUROLOGY-D-20-00293

Safety and efficacy of fluoxetine on functional outcome after acute stroke (AFFINITY): a randomised, double-blind, placebo-controlled trial.

\section{AFFINITY Trial Collaboration*}

*Members of the writing group are listed at the end of the Article;

all members of the AFFINITY trial Collaboration are listed in the appendix.

Summary: $\quad 345$ words

Research in context: 390 words

Text: $\quad 4597$ words

References: $\quad 30$

Tables: $\quad 3$

Figures: $\quad 2$

Supplementary appendix:

Tables: $\quad 5$

Figures: $\quad 3$

Correspondence to:

Professor Graeme J. Hankey

Professor of Neurology, Medical School, Faculty of Health and Medical Sciences,

The University of Western Australia

Room 222, Harry Perkins Institute of Medical Research

QQ Building, QEII Medical Centre,

6 Verdun Street, Nedlands, Perth, Western Australia,

Australia 6009

Email: graeme.hankey@uwa.edu.au

Phone: +61861510828 


\section{Summary}

\section{Background}

Trials of fluoxetine for recovery after stroke report conflicting results. The Assessment oF FluoxetINe In sTroke recoverY (AFFINITY) trial aimed to determine if daily fluoxetine for 6 months after stroke improves functional outcome in Australasian and Vietnamese patients.

\section{Methods}

AFFINITY was a randomised, parallel-group, double-blind, placebo-controlled trial conducted in 43 hospital stroke units in Australia ( $n=29)$, New Zealand (4), and Vietnam (10). Eligible patients were adults with a clinical diagnosis of stroke in the previous 2-15 days and a persisting neurological deficit. Patients were randomised via a web-based system using a minimisation algorithm to once daily, oral fluoxetine $20 \mathrm{mg}$ or matching placebo for 6 months. Patients, investigators and outcome assessors were masked to the treatment allocation. The primary outcome was functional outcome, measured by the modified Rankin scale (mRS), at 6 months. The primary analysis was an ordinal logistic regression of the mRS at 6 months, adjusted for minimisation variables. Analyses were according to the patient's treatment allocation. The trial is registered with the ACTRN registry, number 12611000774921.

\section{Findings}

1280 patients were recruited in Australia $(n=532)$, New Zealand $(n=42)$ and Vietnam $(n=706)$ between 11 January 2013 and 30 June 2019; 642 were allocated fluoxetine and 638 placebo. Adherence to trial medication (mean 167 [SD 48] days) was similar between groups. At 6 months, mRS data were available in 624 (97.2\%) patients allocated fluoxetine and $632(99.1 \%)$ placebo. The distribution of mRS categories at 6 months was similar in the fluoxetine and placebo groups (adjusted common odds ratio 0.936, 95\% CI 0.762-1.150; $\mathrm{p}=0.53$ ), and consistent among all pre-defined subgroups. Compared to placebo, patients allocated fluoxetine had more falls $(20$ [3.12\%] vs 7 [1.10\%]; $p=0.02)$, bone fractures $(19$ [2.96\%] vs 6 [0.94\%]; $\mathrm{p}=0.01)$ and epileptic seizures $(10[1.56 \%]$ vs $2[0.31 \%] ; \mathrm{p}=0.04)$ at 6 months.

\section{Interpretation}

Fluoxetine 20mg daily for 6 months after acute stroke did not improve functional outcome and increased the risk of falls, bone fractures, and seizures. These results do not support the use of fluoxetine to improve outcome after stroke. 


\section{Funding}

National Health and Medical Research Council of Australia (Project Grant 1059094). 


\section{RESEARCH IN CONTEXT}

\section{Evidence before this trial}

We undertook a Cochrane systematic review and searched Cochrane and clinical trial registers; MEDLINE, Embase, PubMed, and other biomedical databases; from their inception to 16 July 2018; for randomised controlled trials (RCTs) that recruited stroke patients who had survived up to one year, and randomised them to a selective serotonin reuptake inhibitor (SSRI), at any dose, for any period, and any indication; or to usual care or placebo. We identified 63 RCTs that compared any SSRI with control in 9168 stroke survivors. About half of the trials required patients to have depression. Potential improvements in disability with fluoxetine were only present in trials at high risk of bias. A meta-analysis of the three trials at low risk of bias $(\mathrm{n}=3356$ patients) found no effect of any SSRI compared to control on functional independence (risk ratio [RR] 1.00, 95\% CI 0.91 to $1.09 ; \mathrm{p}=0.99$ ) or disability score (standardised mean difference [SMD] $-0.01,95 \% \mathrm{CI}-0.09$ to $0.06 ; \mathrm{p}=0.75$ ). The evidence before this trial suggests that SSRIs do not improve functional outcome after stroke, but doubt remains because this meta-analysis was dominated by one large trial, the FOCUS (Fluoxetine Or Control under Supervision) trial $(\mathrm{n}=3127)$, in UK patients.

\section{Added value of this trial}

The Assessment oF FluoxetINe In sTroke recoverY (AFFINITY) trial externally validates the FOCUS trial and Cochrane systematic review of RCTs of SSRIs for stroke recovery in an independent population of Australasian and Vietnamese stroke patients, reinforcing the conclusion that fluoxetine does not improve functional outcome after stroke. The AFFINITY trial also adds further data regarding the potential hazards of treating acute stroke patients with fluoxetine $20 \mathrm{mg}$ daily for 6 months, including increased risks of falls, fractures, and seizures.

\section{Implications of all the available evidence}

For clinicians, SSRIs should not be prescribed routinely to improve functional outcome after stroke because they are ineffective and increase serious adverse events. For researchers, a pooled analysis of individual patient data from completed RCTs of SSRIs for stroke recovery is needed to examine the effects of SSRIs in specific patient subgroups, such as those with hemiparesis, severe stroke, and cognitive impairment; and on specific outcomes, such as the mRS, motor domains of the Stroke Impact Scale, falls, fractures and seizures. Until these results are available, further trials of fluoxetine for stroke recovery are not recommended. 


\section{Introduction}

Stroke is the second leading cause of disability-adjusted life years globally. ${ }^{1,2}$ Fluoxetine, a selective serotonin re-uptake inhibitor (SSRI), may improve neurological recovery and reduce disability after stroke. Fluoxetine exerts neuro-protective and neuro-regenerative effects in preclinical models of acute brain ischaemia. ${ }^{3,4}$ The FLuoxetine for motor recovery After acute ischaeMic strokE (FLAME) trial found that, in 118 patients with moderate to severe motor deficits, fluoxetine $20 \mathrm{mg}$ once daily significantly improved motor recovery after 3 months. ${ }^{5}$ A Cochrane systematic review of 52 randomised controlled trials (RCTs) of SSRIs for stroke recovery in 4059 patients concluded that SSRIs may improve disability but, given methodological limitations and heterogeneity of the studies, more definitive trials were required. ${ }^{6}$

Hence, our international collaboration undertook three trials of fluoxetine for recovery after stroke in the United Kingdom (Fluoxetine Or Control Under Supervision [FOCUS]), Sweden (Efficacy of Fluoxetine-A Randomised Controlled Trial in Stroke [EFFECTS]) and Australia, New Zealand and Vietnam (Assessment oF FluoxetINe In sTroke recoverY [AFFINITY]). ${ }^{7,8}$ The FOCUS trial $(n=3127)$ reported that fluoxetine $20 \mathrm{mg}$ daily for 6 months after stroke did not improve functional outcome as measured by the modified Rankin scale (mRS), but reduced depression and increased bone fractures. ${ }^{9}$ The latter results were consistent with the reported effectiveness of fluoxetine as an antidepressant, ${ }^{10}$ and increased risk of fractures in older people taking SSRIs. ${ }^{11,12}$ However, as only two thirds of FOCUS patients adhered to trial medication for at least 150 of the prescribed 180 days, a modest, but important, effect of fluoxetine on functional outcome may have been missed. ${ }^{9}$ Moreover, as $96 \%$ of patients in FOCUS were white, the results may not be generalisable outside the UK. ${ }^{9}$ Hence, the AFFINITY and EFFECTS trials continued recruitment until 30 June, 2019.

Herein, we report the results of the AFFINITY trial, which aimed to evaluate whether a 6month course of fluoxetine is safe and effective, compared to placebo, for improving functional outcome after recent stroke in an ethnically diverse population. The EFFECTS trial results are reported in a parallel publication. ${ }^{13}$

\section{Methods}

Study design and participants 
AFFINITY was a randomised, parallel group, double-blind, placebo-controlled clinical trial conducted in 43 hospital stroke units in Australia ( $n=29)$, New Zealand (4), and Vietnam (10). The trial protocol (appendix) was approved by the Royal Perth Hospital Ethics Committee on 24 February, 2012 (approval number EC2011/131), and subsequent amendments to the protocol were also approved. All participating sites received approval from their ethics committee and institutional review board. The trial protocol ${ }^{7}$ and statistical analysis plan ${ }^{8}$ were published before recruitment stopped.

Eligible patients were adults (aged $\geq 18$ years) with a clinical diagnosis of acute stroke within the previous 2-15 days, brain imaging consistent with ischaemic or haemorrhagic stroke, and a persisting neurological deficit that produced a mRS score $\geq 1$. Patients were excluded if there was any definite indication for fluoxetine, or contraindication to fluoxetine (e.g. history of epilepsy, bipolar disorder, drug overdose, fluoxetine allergy, or recent medication that could interact with fluoxetine; or biochemical evidence of hepatic impairment [serum alanine aminotransferase > $120 \mathrm{U} / \mathrm{l}$ ], renal impairment [creatinine > $180 \mu \mathrm{mol} / \mathrm{l}$ or estimated glomerular filtration rate $(\mathrm{eGFR})<30 \mathrm{ml} / \mathrm{min} / 1.73 \mathrm{~m}^{2}$ ], or hyponatremia [sodium < $125 \mathrm{mmol} / \mathrm{L}]$ ); if patients were unlikely to be available for follow-up during the subsequent 12 months; if patients had another life-threatening illness that would make 12-month survival unlikely [e.g. terminal malignancy]; if women were pregnant, breast-feeding or of childbearing age and not using contraception; or if patients were enrolled in another clinical trial of an investigational medicinal product (IMP) or device.

Written informed consent was obtained from each patient or, if the patients were unable to provide consent, from their legally approved surrogate.

\section{Randomisation and masking}

The patient's clinician entered the patient's baseline data (table 1) into a secure, passwordprotected, centralised, web-based randomisation system which checked the data for completeness and consistency and generated a unique study identification number and treatment pack number corresponding to fluoxetine or placebo in a 1:1 ratio. A minimisation algorithm ${ }^{14}$ was used to achieve balance between the treatment groups in four predictors of the primary outcome (mRS): time after stroke onset (2-8 vs 9-15 days), presence of a motor deficit (National Institutes of Health Stroke Scale [NIHSS] questions 5 and 6), presence of aphasia 
(NIHSS question 9), and probability of survival free of dependency (mRS 0-2) at 6 months $(0.00$ to $\leq 0 \cdot 15$ vs $>0 \cdot 15-1.00)$ calculated using a validated prognostic model comprising six baseline variables (age, living alone before the stroke, independent in activities of daily living before the stroke, and able to talk, lift both arms off the bed, and walk unassisted at randomisation). ${ }^{15}$

All patients, carers, investigators, and outcome assessors were masked to the allocated treatment by use of placebo capsules that were visually identical to the fluoxetine capsules even when broken open.

\section{Procedures}

Fluoxetine $20 \mathrm{mg}$ capsules or matching placebo capsules were administered orally, once daily, for 6 months. If patients were unable to swallow, the capsules were broken open and contents administered via an enteral feeding tube.

Siegfried Malta Ltd, Hal Far, Malta, manufactured the capsules containing fluoxetine 20mg according to Good Manufacturing Practice (certificate MT/008HM/2017). Arena Pharmaceuticals $\mathrm{GmbH}$, Zofingen, Switzerland, packed the capsules for Amneal Pharmaceuticals Pty Ltd, South Yarra, Australia, which was the Therapeutic Goods Administration (TGA) licence holder (sponsor) for the finished product in Australia. Pharmaceutical packaging professionals (PPP) Pty Ltd, Port Melbourne, Australia purchased the fluoxetine capsules and manufactured the matching placebo capsules. PPP packaged the trial medication in patient kits, labelled the bottles with trial-specific treatment codes (fluoxetine or placebo), and packaged, stored and distributed the medication. The patient kits in Australian and New Zealand comprised two bottles, each containing 110 capsules, which were dispensed at randomisation and day 90. An extra 20 capsules were a reserve in the event of any delay in attending the day 90 follow-up, or any loss or spillage of capsules. For patients in Vietnam, the kits comprised 6 bottles of trial medication, each containing 35 capsules. One bottle was dispensed at randomisation, two bottles at day 28 , and three bottles at day 90 . The TGA of the Australian government's Department of Health approved the export of trial medication to New Zealand and Vietnam (approval Ref No: EX17/336513).

All patients received organised, interdisciplinary care and rehabilitation in stroke units. 
Patients recruited in Australia and New Zealand were assessed by site investigators at 28 days (1 month) and 90 days ( 3 months) post-randomisation in the hospital ward or outpatient clinic or via telephone or email; or, failing that, by a study nurse at the patient's residence. Followup at 180 days ( 6 months) was by postal questionnaire or telephone by trained staff in the trial coordinating centre in Perth, Australia. Patients recruited in Vietnam were assessed by the site investigator at 28,90 and 180 days post-randomisation in the hospital ward or outpatient clinic, or via telephone or email; or, failing that, at the patient's residence. If the patient was unable to complete the assessments, assistance was sought from their proxy (next of kin, close family member or carer). Each assessment recorded the primary outcome (mRS), secondary outcomes (table 2), safety and adverse events (table 3), all current medications, and adherence to trial medication. Serum sodium, eGFR, and liver function were measured at the 28 day follow-up visit if clinically appropriate. Adherence to trial medication was assessed by asking: 'On average, since the last follow-up, how many times per week was the trial medication taken? ' 0 , 1-2, 3-4, 5-6 or 7 times per week'; and by pill counts and collection of returned trial bottles. Bottle and pill counts were conducted by hospital trial pharmacists, and entered on a drug accountability form. Any interruption to trial medication was recorded as temporary or permanent, together with the dates and reasons for stopping and re-starting.

If patients developed new depression requiring treatment during the trial treatment period, the protocol recommended continuation of trial medication and consideration of nonpharmacological (e.g. psychological) interventions. If antidepressant medication was necessary, referral to a psychiatrist was recommended for consideration of potential interactions of any new medication with fluoxetine and risks of serotonin toxicity.

There were 57 protocol violations in 56 patients (4.4\%): 20 (1.6\%) patients were prescribed open-label fluoxetine; $8(0.6 \%)$ were prescribed another SSRI; $16(1.3 \%)$ lost their trial medication or did not take it as prescribed; $4(0.3 \%)$ took medications that could interact with fluoxetine (e.g. antipsychotic, tramadol); 7 (0.5\%) patients were $>14$ days late for scheduled follow-up; one patient enrolled in another trial was ineligible, and four patients had a final diagnosis other than stroke (table 1). Emergency unblinding was not necessary for any patient. 


\section{Outcomes}

The primary outcome was functional status, as measured by the mRS, at 6 months after randomization. ${ }^{16}$ The mRS is an ordinal scale which assigns patients to seven ordered, but not equally spaced, levels of functional ability, ranging from 0 (symptom free) to 6 (dead).

Secondary outcomes at 6 months were survival, depression (PHQ-9 score $\geq 15^{17}$ ), cognition (Telephone Interview for Cognitive Status [TICSm $]^{18}$ ), communication, motor function, overall health status (Stroke Impact Scale [SIS] version 3.0 ${ }^{19}$ ), fatigue (vitality subscale of the SF-36 ${ }^{20,21}$ ) and health-related quality of life (HRQoL) using the EQ-5D-5L. ${ }^{22}$ A new diagnosis of depression requiring treatment with antidepressants was assessed at 1, 3 and 6 months by asking patients if they had been diagnosed with depression since their previous assessments, and verifying the diagnosis and treatment with their clinician.

Serious adverse events during follow-up included recurrent stroke (ischaemic or haemorrhagic), acute coronary syndromes, upper gastrointestinal bleeding requiring blood transfusion and/or endoscopy, other major bleeding (subdural, extradural, ocular, lower gastrointestinal) requiring blood transfusion or procedural intervention, falls with injury, new bone fractures, epileptic seizures, symptomatic hypoglycaemia (blood glucose $<3 \mathrm{mmol} / \mathrm{l}$ ), symptomatic hyperglycaemia (blood glucose $>22 \mathrm{mmol} / \mathrm{l}$ ), new hyponatraemia (blood sodium $<125 \mathrm{mmol} / \mathrm{l})$, attempted suicide or self-harm, and death.

\section{Statistical analysis}

A detailed statistical analysis plan was formulated and published before recruitment was completed and without awareness of any unblinded data. ${ }^{8}$

We estimated from other concurrent studies of patients with acute stroke that $42.2 \%$ of patients assigned placebo would be functionally independent (mRS 0-2) at 6 months after randomisation. ${ }^{23,24}$ We calculated the odds ratio (OR) of functional independence (mRS 0-2) with fluoxetine vs placebo in the FLAME study ${ }^{5}$ to be 3.57 (95\%CI: 1.2 to 10.6). We considered a conservative estimate of the effect of fluoxetine may be toward the lower $95 \% \mathrm{CI}$ of the OR estimate reported in FLAME (e.g. OR 1.34). If fluoxetine increased the proportion of patients who were functionally independent at 6 months by an OR of 1.34, from $42.2 \%$ 
(placebo) to $49.4 \%$ (fluoxetine), this would be clinically important and consistent with our Cochrane review. ${ }^{6}$ Assuming a common OR of 1.34 for each cut-point across the mRS (e.g. 0 vs $1-6,0-1$ vs $2-6$, etc) in the proportional odds logistic model, we estimated that the trial would require 1600 patients to have $90 \%$ power, if up to $10 \%$ of patients dropped out before final follow-up (i.e. 1440 patients with primary outcome data). ${ }^{8,25}$

All analyses, including primary and secondary outcomes and adverse events, were by intentionto-treat, according to the treatment allocation. A secondary safety analysis was undertaken according to the treatment patients received rather than what they were randomly allocated.

The primary analysis was an ordinal analysis of the mRS scores at 6 months in each treatment group using ordinal logistic regression and after adjusting for the baseline factors included in the minimisation algorithm. ${ }^{8}$ The ordinal analysis of $\mathrm{mRS}$ was undertaken by treatment allocation, under the assumption of proportional odds in the model. The result was expressed as a common OR (less than 1.0 favoured placebo) and its $95 \%$ confidence interval (CI). We also performed 6 binary unadjusted logistic regressions, each corresponding to the 6 possible dichotomisations of scores on the mRS.

Secondary analyses compared the following outcomes at 6 months in each treatment group: survival, depression (changes in PHQ-9 scores and proportion with PHQ-9 $\geq 15$ ), ${ }^{17}$ cognition (TICSm scores), ${ }^{18}$ communication (SIS), ${ }^{19}$ motor function (SIS), ${ }^{19}$ overall health status (SIS), ${ }^{19}$ HRQoL (EQ-5D-5L), ${ }^{22}$ new diagnosis of depression requiring treatment with antidepressants, fatigue (vitality domain of the SF-36) ${ }^{20,212}$, trial medication adherence and cessation, and serious adverse events. The frequencies of categorical outcome events in each group were compared using Fisher's exact test. For continuous outcomes, the mean or median in each group, depending on the distribution, were calculated with measures of dispersion (standard deviation $[\mathrm{SD}]$ or inter-quartile range [IQR]). The probability that outcomes in the fluoxetine group were significantly different from the placebo group were calculated as p-values.

Pre-specified subgroup analyses of the effect of fluoxetine vs placebo on the primary outcome were undertaken for country of randomisation (Australia/New Zealand vs Vietnam), age ( $\leq 70$ vs >70 years), time from stroke onset to randomisation (2-8 vs 9-15 days), stroke pathology (ischaemic vs haemorrhagic), stroke severity (NIHSS scores $<$ median [0-5] vs > median [>5]), 
motor deficit (present vs absent), aphasia (present vs absent), probability of survival free of dependency ( 0.00 to $\leq 0.15$ vs $>0.15$ to 1.00 ), self-reported depression at baseline, and source of informed consent. ${ }^{8}$

We also undertook pre-specified per-protocol analyses, which sequentially excluded subgroups of patients who did not meet our eligibility criteria or had incomplete adherence to the trial medication.

Post-hoc sensitivity analyses of the primary outcome were undertaken to evaluate the possible effect of including patients who were lost to follow-up. We tested the robustness of the results by assuming two extreme imputation scenarios: one favouring fluoxetine where all patients with missing $\mathrm{mRS}$ data were imputed a score of 0 in the fluoxetine arm and a score of 6 in the placebo arm, and another scenario favouring placebo where the imputation was reversed.

Statistical analyses were undertaken with SAS, version 9.4

An independent data monitoring committee (DMC) oversaw the study. The unmasked trial statistician (Q Yi) prepared analyses of the accumulating data, which the DMC reviewed in confidence at least annually.

The trial is registered with the Australian New Zealand Clinical Trials Registry, number ACTRN12611000774921.

\section{Role of the funding source}

The funder of the study had no role in study design, data collection, data analysis, data interpretation, or writing of the report. The corresponding author had full access to all the data in the study and had final responsibility for the decision to submit for publication.

\section{Results}

A total of 1280 patients consented and were randomised at 43 sites in Australia, New Zealand and Vietnam between 11 January, 2013 and 30 June, 2019. Recruitment was terminated before the target of 1600 patients was reached because funding expired on 31 December 2019. 
642 patients were randomly allocated to fluoxetine and 638 to placebo. One patient did not meet our eligibility criteria after discovery of participation in another trial of an IMP. In four patients, the diagnosis was later revised to be non-stroke (table 1).

Baseline characteristics in the two groups were balanced (table 1)

By 6 months, $22(1.7 \%)$ patients had withdrawn consent for follow-up and $2(0.2 \%)$ were lost to follow-up (fig 1). There was no difference in the methods of follow-up between groups (appendix table 1, p8). Trial medication was started in 1273 patients, and temporarily and permanently stopped in 158 and 208 patients respectively (appendix, table 2, p9). There was no significant difference between groups in temporary and permanent discontinuation of trial medication (appendix table 2, p9) or time to permanent discontinuation ( $\mathrm{p}=0.75$; appendix figure 1, p14). There were also no differences between groups in compliance with trial medication (appendix table 3, p10). The mean duration of trial treatment was 167 days (SD 48.1) days.

The mRS at 6 months was assessed and analysed in $624(97.2 \%)$ patients allocated fluoxetine and $632(99.1 \%)$ placebo. An ordinal comparison of the distribution of patients across $\mathrm{mRS}$ categories at 6 months, adjusted for variables in the minimisation algorithm, was similar in both groups (common OR 0.936, 95\% CI 0.762-1.150; $\mathrm{p}=0.53$; figure 2). A common OR $<1.0$ favours placebo. The unadjusted analysis produced similar results (common OR 0.966, 95\%CI $0.790-1.181 ; \mathrm{p}=0.74$; appendix table $4, \mathrm{p} 11)$. The assumption of proportional odds in the model of $\mathrm{mRS}$ by treatment was upheld in the score test for proportional odds assumption $(\mathrm{p}=0.44$ unadjusted). Dichotomised mRS scores were also not significantly different between groups for mRS 0-2 vs 3-6 (unadjusted OR 0.855, 95\%CI 0.670-1.091; $\mathrm{p}=0.21$; post-hoc adjusted $\mathrm{OR}=0.823,0.628-1.077 ; \mathrm{p}=0.16$ ), or other dichotomies of the mRS (appendix table 4, p11).

Analysis of the primary outcome showed no significant interactions or modification of the effect of fluoxetine across several pre-specified subgroups (appendix figure 2, p15).

Secondary efficacy outcomes at 6 months are shown in table 2. Patients allocated fluoxetine had higher scores in the SIS domain of mood and emotional control compared to placebo ( $\mathrm{p}=0.003$ ), but there were no significant differences between treatment groups in the other 10 domains of the SIS (including measures of motor function [strength, hand ability, mobility] and daily activities), other assessment scales, or death. There was a reduction in new diagnoses 
of post-stroke depression which was not statistically significant (33 [5.14\%] fluoxetine vs 46 [7.21\%] placebo; absolute risk difference $2.07 \%$; $95 \%$ CI $-0.57 \%$ to $4.41 \%]$ ).

Adverse events at 6 months are shown in table 3. Compared to patients allocated placebo, those allocated fluoxetine had more falls causing injury (20 [3.12\%] vs 7 [1.10\%]; difference $2.02 \%$ [95CI: 0.45-3.59]; $\mathrm{p}=0.02)$, bone fractures (19 [2.96\%] vs 6 [0.94\%]; difference $2.02 \%$ [0.51$3.53] ; \mathrm{p}=0 \cdot 01)$ and epileptic seizures (10 [1.56\%] vs 2 [0.31\%]; difference $1.24 \%$ [0.19-2.30]; $\mathrm{p}=0.04)$. There were no significant differences between groups in other events, including survival ( $\mathrm{p}=0.71$, appendix, figure $3, \mathrm{p} 16)$. Trial medication was stopped by 68 patients (27 allocated fluoxetine, 41 placebo) due to a suspected adverse reaction to the medication. No patients required a reduction in dose of trial medication (e.g. alternate daily) and there were no treatment-related deaths.

The primary results were not altered by sensitivity analyses confined to patients who adhered to the trial protocol and allocated treatment (appendix table 5, p12).

A post-hoc analysis, imputing missing $\mathrm{mRS}$ data under two extreme scenarios, also produced non-significant results for the most extreme scenarios in favour of fluoxetine (unadjusted OR 1.082, 95\%CI 0.887-1.320; $\mathrm{p}=0.44$; adjusted OR 1.054, 0.861-1.291; $\mathrm{p}=0.61$ ), and in favour of placebo (unadjusted OR 0.860, 0.705-1.050; p=0.14; adjusted OR 0.833, 0.680-1.020; p=0.09).

\section{Discussion}

The main finding of the AFFINITY trial was that adding fluoxetine $20 \mathrm{mg}$ daily for 6 months after acute stroke to interdisciplinary stroke unit care did not improve functional outcome at 6 months in an ethnically diverse population. Other major findings were that fluoxetine increased falls, fractures, and epileptic seizures at 6 months.

Key strengths of the trial are that it was undertaken in stroke units throughout Australia, New Zealand and Vietnam where the AFFINITY trial medication was added to best-practice, comprehensive interdisciplinary stroke care and rehabilitation. Several potential sources of systematic error (bias) in the assessment of fluoxetine vs placebo were minimised. Systematic pre-treatment differences in comparator groups (selection bias) were minimised by concealed, central, web-based randomisation. Adherence to trial medication was high and similar between treatment groups (performance bias). Systematic differences between groups in other care 
provided (performance bias) and reporting and assessment of outcome events (observer detection bias) were minimised by masking of patients, investigators and adjudicators to the allocated treatment. Follow up for the primary outcome was high and there was no difference between groups in withdrawals from treatment (attrition bias). Random error was reduced to some extent by almost complete follow-up of a large number of patients $(n=1256,98 \%)$, which was a higher proportion than planned in our sample size calculations (90\% of $1600 ; n=1440)$. The inclusion of an international mix of ethnic groups managed in different health care systems, and a comprehensive array of secondary outcome measures, including cognition, mood and motor scales, support the external validity (generalisability) of the trial results.

Potential limitations of the trial include our failure to recruit the target sample size of 1600 patients due to funding constraints (1280 patients recruited; 1256 with primary outcome data vs 1440 planned to have primary outcome data). We also failed to recruit a larger number of patients with severe, disabling stroke. Hence, the proportion of patients assigned placebo who recovered functional independence (mRS 0-2) at 6 months was higher $(n=458,72 \%)$ than estimated in our sample size calculations (42\%). The dose of fluoxetine was $20 \mathrm{mg}$ once daily because this was the dose reported to be effective in the FLAME trial ${ }^{5}$ and used in other fluoxetine trials for stroke recovery, ${ }^{6}$ and is less likely to cause adverse effects than higher doses. However, we did not test higher doses of fluoxetine. Our measures of adherence to trial medication by self-report and capsule-counting were prone to error (e.g. the absence of tablets in the bottles returned to investigators may not necessarily mean adherence to taking the tablets) and therefore, our estimates of adherence and compliance may be inflated. However, there was no difference between groups in reported adherence to, and discontinuation of, trial medication. The nature and degree of adjunctive rehabilitation was not documented because that would have added complexity and potential measurement error to this pragmatic trial. However, all patients were admitted to stroke units where organised interdisciplinary assessment, and rehabilitation as required, was provided as standard practice. The nature and intensity of all rehabilitation interventions were likely to be balanced between the treatment groups, in the same way that all baseline variables were balanced between groups, due to the randomisation process and double-blind trial treatment allocation. There was a slight difference in ascertainment of mRS status at 6 months between groups (fluoxetine $n=624,97.2 \%$ vs placebo $n=632,99.0 \%$ ) but sensitivity analyses using imputations led to consistent conclusions. Our primary measure of efficacy was a broad measure of functional outcome which may not 
be sensitive to changes in measures of specific neurological functions, However, we also measured 11 domains of the SIS, including measures of motor function (strength, hand ability, mobility), physical function, and daily activities, and found no effect of fluoxetine on any of these measures. The mRS may be less sensitive to change in patients with less severe stroke but there was no evidence of an effect of fluoxetine on the $\mathrm{mRS}$ in patients with more severe stroke (NIHSS > 5; appendix figure 2, p15), and no effect of fluoxetine on any secondary outcome except mood and emotional control.

The AFFINITY trial was smaller than the FOCUS trial ${ }^{9}$ but both trials recruited patients of similar sex (women 38\%), stroke severity (median NIHSS=6) and at a similar time (one week, mean) after stroke onset. The AFFINITY trial population was a unique mix of Vietnamese $(\mathrm{n}=727,57 \%)$ and Australasians $(\mathrm{n}=553,43 \%)$, whereas the FOCUS population was predominantly Caucasian ( $\mathrm{n}=2988 ; 96 \%$ ). Patients in AFFINITY were also younger (mean age 64 years AFFINITY, vs 71 years in FOCUS), and more likely to be married ( $\mathrm{n}=926,72 \%$ vs $\mathrm{n}=1725,55 \%)$, living with someone else $(\mathrm{n}=1120,87 \%$ vs $\mathrm{n}=2091,67 \%)$, employed $(\mathrm{n}=531$, $41 \%$ vs $n=691,22 \%)$, and independent before their stroke ( $n=1264,99 \%$ vs $n=2866,92 \%)$ compared to FOCUS. Adherence to trial medication was higher in AFFINITY than FOCUS; $34(5.4 \%)$ patients assigned fluoxetine and $30(4.8 \%)$ placebo stopped trial medication within the first 90 days, whereas in FOCUS, 143 (9\%) patients assigned fluoxetine and $122(8 \%)$ placebo stopped trial medication within the first 90 days. Despite these differences, the results of the AFFINITY trial almost replicate those of the FOCUS trial, ${ }^{9}$ supporting the internal and external validity of both trials. Furthermore, the EFFECTS trial of fluoxetine vs placebo in 1500 stroke patients in Sweden also reports similar results to FOCUS and AFFINITY. ${ }^{13}$ Moreover, the results of the FOCUS, AFFINITY and EFFECTS trials are all consistent with the totality of evidence from all RCTs of SSRIs for stroke recovery, ${ }^{26}$ and all RCTs specifically testing fluoxetine. ${ }^{27}$ Collectively, these trials provided compelling evidence that fluoxetine does not improve functional outcome after stroke.

The outstanding inconsistency among all the RCT evidence is the FLAME trial, which did report a significant benefit of fluoxetine on motor recovery after stroke. ${ }^{5}$ The FLAME trial differed from AFFINITY, FOCUS and EFFECTS in that it was a phase II trial of fluoxetine 20 $\mathrm{mg}$ daily vs placebo in a select population of 118 patients with recent (5-10 days) ischaemic stroke and a moderate to severe hemiparesis or hemiplegia, defined by a Fugl-Meyer motor 
scale (FMMS) score $\leq 55 .{ }^{5}$ The FMMS ranges from 0 (hemiplegia) to 100 points (normal motor performance), with 66 points for the upper extremity and 34 points for the lower extremity. Patients enrolled in FLAME had more severe strokes (mean baseline NIHSS 13 vs median NIHSS 6 in AFFINITY). ${ }^{5}$ Somatosensory and other neurological deficits that may influence recovery were not reported. The primary outcome, the mean change in FMMS scores between randomisation and day 90 , was greater with fluoxetine than placebo (34.0 points fluoxetine vs 24.3 points placebo; difference 9.8 points, $95 \%$ CI: 3.4 to $16.1, \mathrm{p}=0.003)$. The proportion of functionally independent patients (mRS scores 0-2) at day 90 was also higher with fluoxetine than placebo $(n=15,26 \%$ vs $n=5,9 \% ; p=0.02)$, but there were no differences between fluoxetine and placebo for other mRS categories. The FLAME trial result may be a falsepositive due to random error (chance), as only 57 patients were treated with fluoxetine and followed-up to 90 days, ${ }^{5}$ and there is large variation in spontaneous motor recovery after acute stroke. ${ }^{28}$ Alternatively, the FLAME trial result may a true-positive, and fluoxetine may indeed improve motor recovery in patients with severe motor impairment. The AFFINITY trial did not include a large number patients with severe hemiparesis and did not measure motor recovery by the FMMS, but did measure motor functions as domains within the SIS and found no effect of fluoxetine. Our planned individual patient data meta-analysis of the FOCUS, AFFINITY and EFFECTS trials ${ }^{8}$ will constitute a larger number of stroke patients with severe motor impairments and promises to enable a more reliable analysis of the effect of fluoxetine, vs placebo, on the mRS and motor domains of the SIS at 6 months in this subgroup.

The AFFINITY trial also confirms the FOCUS trial finding that long-term fluoxetine in stroke patients has hazards, increasing the risk of bone fractures. ${ }^{9}$ We also found that fluoxetine significantly increased the risk of falls with injury and epileptic seizures in stroke patients. The FOCUS trial reported similar, but not significant, increases in falls with injury and seizures in patients allocated fluoxetine. ${ }^{9}$ The AFFINITY and FOCUS trials collectively provide robust evidence about the effect of an SSRI on the incidence of falls causing injury, and fractures, increasing the absolute risk of each by about $2 \%$ over 6 months among patients with recent stroke.

Although fluoxetine is more effective than placebo in treating major depressive disorders, ${ }^{10}$ and reduced the rate of new depression in the FOCUS, ${ }^{9}$ and other trials, ${ }^{27,29}$ we observed only improved mood and emotional control, as measured by the SIS, at 6 months with fluoxetine; 
the numerically lower rate of post-stroke depression with fluoxetine vs placebo was not statistically significant. We believe we lacked statistical power to show a significant effect of fluoxetine on post-stroke depression because the absolute rates of depression in both groups in AFFINITY were substantially lower (less than half) than in FOCUS, possibly from underreporting, particularly in Vietnam where the reporting of changes in mood may be affected by the cultural setting. ${ }^{30}$

In summary, the AFFINITY trial reinforces the conclusion of a recent Cochrane review that SSRIs are not effective at improving functional outcome after stroke. It also confirms that fluoxetine may improve mood but have important adverse effects, particularly bone fractures. A planned individual patient data meta-analysis of the AFFINITY, FOCUS and EFFECTS trials will produce greater precision in the estimates of the effects of fluoxetine on functional outcome in important patient subgroups. ${ }^{8}$ 


\section{References}

1. Hankey GJ. Stroke. Lancet. 2017; 389: 641-654.

2. GBD 2016 Stroke Collaborators. Global, regional, and national burden of stroke, 1990-2016: a systematic analysis for the Global Burden of Disease Study 2016. Lancet Neurol. 2019; 18: 439-458.

3. Lim CM, Kim SW, Park JY, Kim C, Yoon SH, Lee JK. Fluoxetine affords robust neuroprotection in the post-ischemic brain via its anti-inflammatory effect. J Neurosci Res. 2009; 87: 1037-45.

4. Wang JW, David DJ, Monckton JE, Battaglia F, Hen R. Chronic fluoxetine stimulates maturation and synaptic plasticity of adult-born hippocampal granule cells. J Neurosci. 2008; 28:1374-84.

5. Chollet F, Tardy J, Albucher JF, et al. Fluoxetine for motor recovery after acute ischaemic stroke (FLAME): a randomised placebo-controlled trial. Lancet Neurol 2011; 10:123-30.

6. Mead GE, Hsieh CF, Lee R, et al. Selective serotonin reuptake inhibitors (SSRIs) for stroke recovery. Cochrane Database Syst Rev 2012; 11: CD009286.

7. Mead G, Hackett ML, Lundstrom E, Murray V, Hankey GJ, Dennis M. The FOCUS, AFFINITY and EFFECTS trials studying the effect(s) of fluoxetine in patients with a recent stroke: a study protocol for three multicentre randomised controlled trials. Trials 2015; 16: 369.

8. Graham C, Lewis S, Forbes J, et al. The FOCUS, AFFINITY and EFFECTS trials studying the effect(s) of fluoxetine in patients with a recent stroke: statistical and health economic analysis plan for the trials and for the individual patient data metaanalysis. Trials 2017; 18: 627.

9. FOCUS Trial Collaboration. Effects of fluoxetine on functional outcomes after acute stroke (FOCUS): a pragmatic, double-blind, randomised, controlled trial. Lancet. 2019; 393: 265-274.

10. Cipriani A, Furukawa TA, Salanti G, et al. Comparative efficacy and acceptability of 21 antidepressant drugs for the acute treatment of adults with major depressive disorder: a systematic review and network meta-analysis. Lancet. 2018; 39:13571366. 
11. Coupland C, Dhiman P, Morriss R, Arthur A, Barton G, Hippisley-Cox J. Antidepressant use and risk of adverse outcomes in older people: population based cohort study. BMJ. 2011; 343: d4551.

12. Brännström J, Lövheim H, Gustafson Y, Nordström P. Association between antidepressant drug use and hip fracture in older people before and after treatment initiation. JAMA Psychiatry. 2019; 76: 172-179.

13. Lundström E, Isaksson E, Näsman P, et al., Efficacy of fluoxetine - a randomised controlled trial in stroke (EFFECTS). The Lancet Neurology (?in press THELANCETNEUROLOGY-D-20-00292)

14. Altman DG, Bland JM. Treatment allocation by minimisation. BMJ 2005; 330: 843.

15. Counsell C, Dennis M, McDowall M, Warlow C. Predicting outcome after acute and subacute stroke: development and validation of new prognostic models. Stroke. 2002; 33: $1041-1047$

16. Bruno A, Shah N, Lin C, et al. Improving modified Rankin scale assessment with a simplified questionnaire. Stroke. 2010; 41: 1048-1050.

17. Williams LS, Brizendine EJ, Plue L, Bakas T, Tu W, Hendrie H, Kroenke K. Performance of the PHQ-9 as a screening tool for depression after stroke. Stroke. 2005; 36: 635-638.

18. de Jager CA, Budge MM, Clarke R. Utility of TICS-M for the assessment of cognitive function in older adults. International Journal of Geriatric Psychiatry. 2003; 18: $318-324$.

19. Duncan PW, Bode RK, Min Lai S, Perera S; Glycine Antagonist in Neuroprotection Americans Investigators. Rasch analysis of a new stroke-specific outcome scale: the Stroke Impact Scale. Arch Phys Med Rehabil. 2003; 84: 950-63.

20. Mead GE, Lynch J, Greig CA, Young A, Lewis S, Sharpe M. Evaluation of fatigue scales in stroke patients. Stroke. 2007; 38: 2090-2095.

21. Ware JE, Snow KK, Kosinski M, Gandek B. SF-36 health survey: manual and interpretation guide. Boston, MA: New England Medical Center, Health Institute; 1993.

22. Norman R, Cronin P, Viney R. A pilot discrete choice experiment to explore preferences for EQ-5D-5L health states. Appl Health Econ Health Policy. 2013; 11: 287-98. 
23. CLOTS Trials Collaboration. Thigh-length versus below-knee stockings for DVT prophylaxis after stroke: a randomized trial. Ann Int Med 2010; 153: 553-562.

24. IST-3 collaborative group. The benefits and harms of intravenous thrombolysis with recombinant tissue plasminogen activator within $6 \mathrm{~h}$ of acute ischaemic stroke (the third international stroke trial [IST-3]): a randomised controlled trial. Lancet 2012; 379: 2352-63.

25. Howard G, Waller JL, Voeks JH, et al.. A simple, assumption-free, and clinically interpretable approach for analysis of modified Rankin outcomes. Stroke. 2012; 43: 664-9.

26. Legg LA, Tilney R, Hsieh CF, et al. Selective serotonin reuptake inhibitors (SSRIs) for stroke recovery. Cochrane Database Syst Rev. 2019; 11: CD009286.pub3.

27. Mead GE, Legg L, Tilney R, et al. Fluoxetine for stroke recovery: Meta-analysis of randomized controlled trials. Int J Stroke. 2019 Oct 17. doi:

10.1177/1747493019879655. [Online ahead of print] PMID: 31619137 (accessed April 16, 2020).

28. van der Vliet R, Selles RW, Andrinopoulou ER, et al. Predicting Upper Limb Motor Impairment Recovery after Stroke: A Mixture Model. Ann Neurol. 2020; 87: 383393.

29. Kraglund KL, Mortensen JK, Damsbo AG, et al. Neuroregeneration and Vascular Protection by Citalopram in Acute Ischemic Stroke (TALOS). Stroke. 2018; 49: 2568-2576.

30. Steel Z, Silove D, Giao N, et al., International and indigenous diagnoses of mental disorder among Vietnamese living in Vietnam and Australia. Br J Psychiatry, 2009; 194: 326-333. 


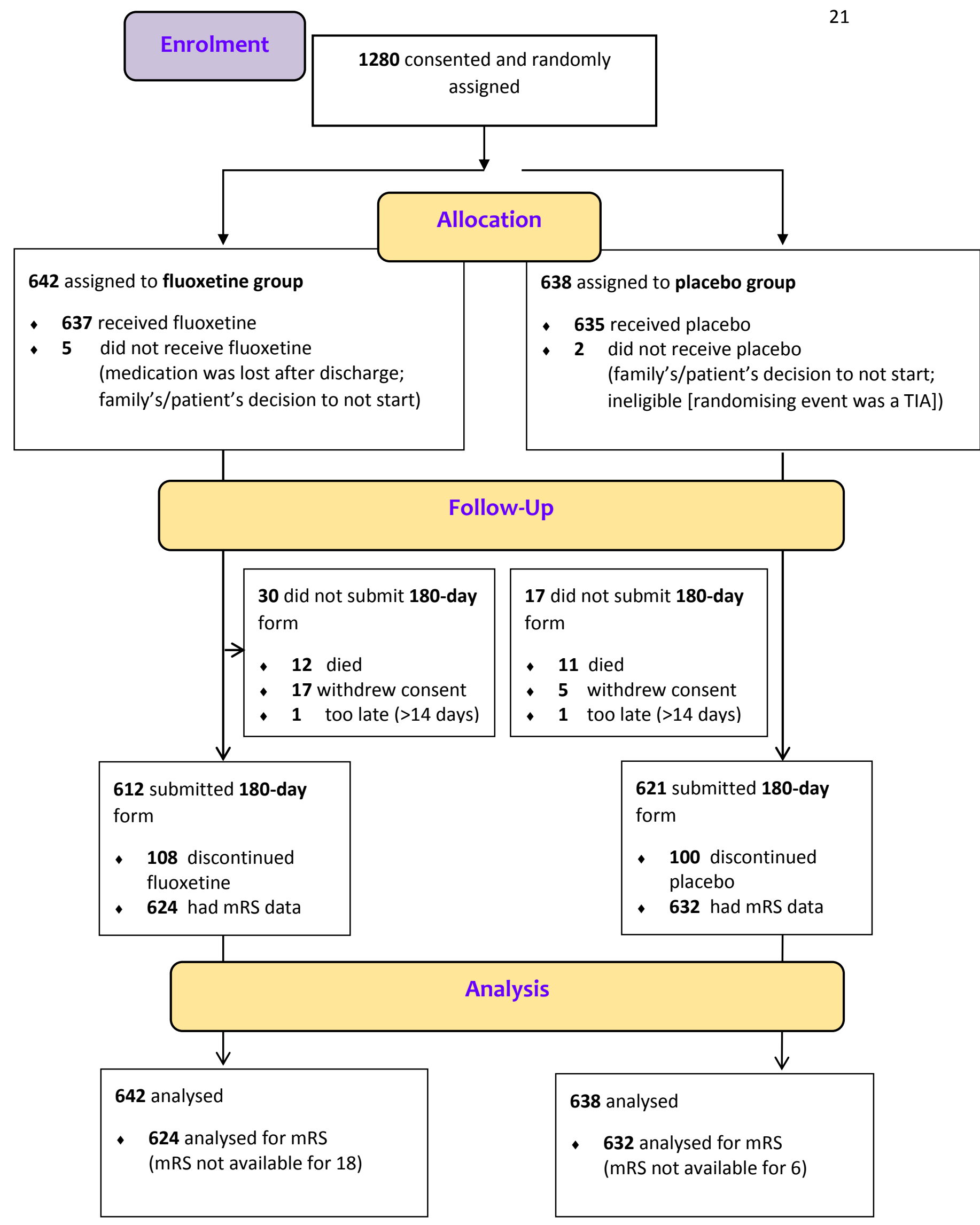

Figure 1: AFFINITY trial profile $m R S=$ modified Rankin Scale 


\section{Figure 2.}

\section{Primary outcome of the distribution of the modified Rankin Scale (mRS) scores at 6 months by treatment group.}

The primary outcome was an assessment of scores across all seven categories of the mRS (ranging from 0 [no symptoms] to 6 [death]), using a shift analysis of the ordinal data. The odds ratio and p-values were calculated with ordinal logistic regression, adjusted for the baseline variables included in the minimisation algorithm (delay between stroke onset and randomization, probability of being alive and independent at 6 months, presence of a motor deficit, presence of aphasia). mRS data at 6 months were available for 624 (97.2\%) patients allocated fluoxetine and $632(99.1 \%)$ allocated placebo. The common odds ratio was 0.936 (95\%CI: 0.762 to 1.150 ), $\mathrm{p}=0.53$; adjusted for baseline minimization variables. A common OR less than 1.0 favoured placebo.

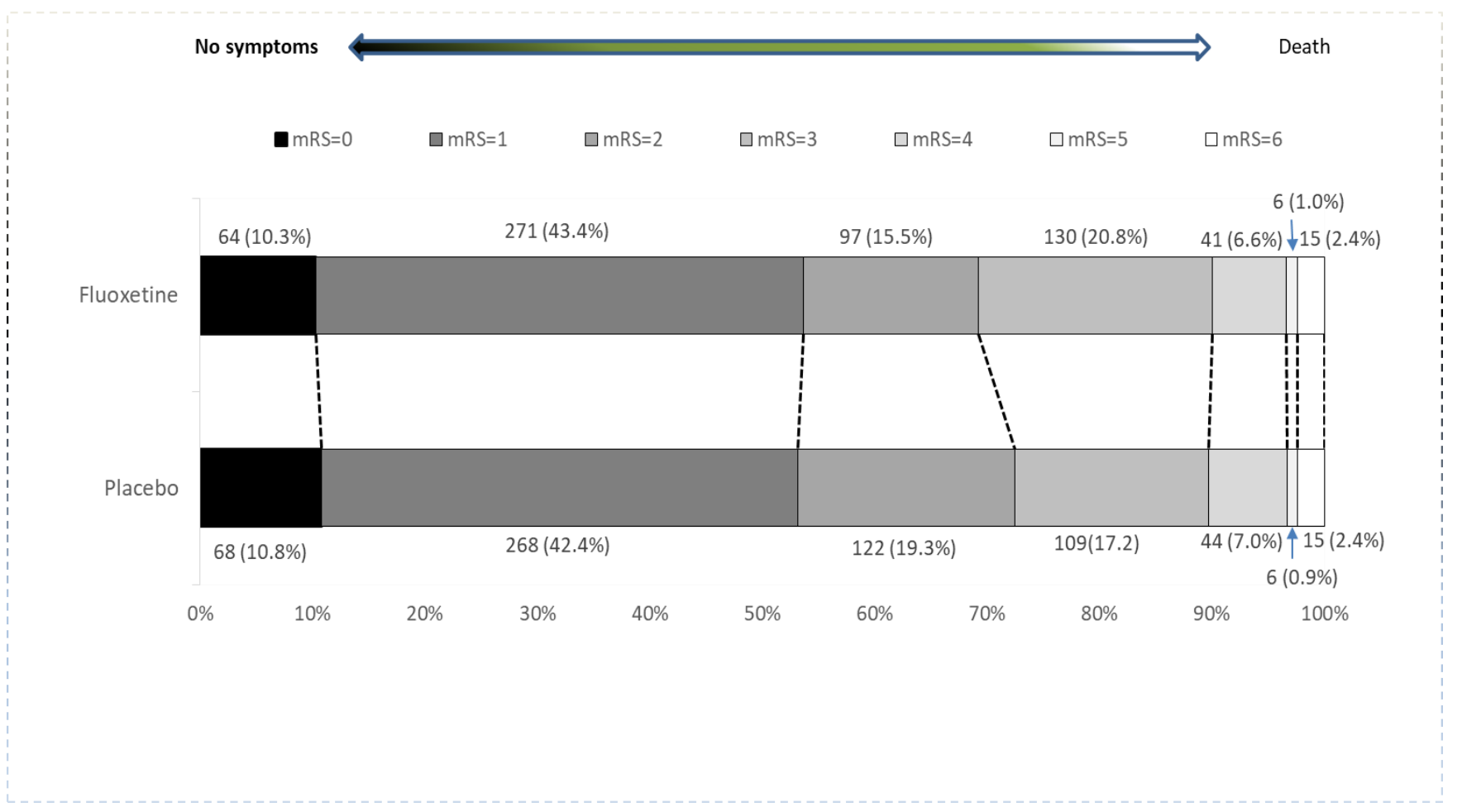




\section{Writing group of the AFFINITY trial Collaboration}

Graeme J. Hankey (Chair)

Professor of Neurology, Medical School, Faculty of Health and Medical Sciences, The University of Western Australia, Perth, Western Australia;

Neurologist, Department of Neurology Sir Charles Gairdner Hospital, Perth, Australia Maree L. Hackett

Professor, The George Institute for Global Health, Faculty of Medicine,

University of New South Wales Sydney;

Professor of Epidemiology, The University of Central Lancashire, United Kingdom.

Osvaldo P. Almeida

Professor of Geriatric Psychiatry, Medical School, Faculty of Health and Medical

Sciences, The University of Western Australia, Perth, Western Australia

Leon Flicker

Professor of Geriatric Medicine, Medical School, Faculty of Health and Medical Sciences, The University of Western Australia, Perth, Western Australia

Geriatrician, Royal Perth Hospital, Perth, Australia

Gillian E. Mead

Professor of Stroke and Elderly Care Medicine, University of Edinburgh, Royal Infirmary, Edinburgh

Martin S. Dennis

Professor of Stroke Medicine, University of Edinburgh, Royal Infirmary, Edinburgh Christopher Etherton-Beer

Associate Professor, The University of Western Australia, Perth, Western Australia Andrew H. Ford

Associate Professor, The University of Western Australia, Perth, Western Australia Laurent Billot

Director, Statistics Division, The George Institute for Global Health, New South Wales, Australia

Associate Professor, Faculty of Medicine, UNSW, Sydney

Stephen Jan

Professor of Health Economics, The George Institute for Global Health, Faculty of Medicine, University of New South Wales, Sydney, Australia

Thomas Lung

The George Institute for Global Health, Sydney

Erik Lundström

Associate Professor, Department of Neuroscience, Neurology, Uppsala University, Uppsala, Sweden. 
Craig S. Anderson

Professor of Neurology and Epidemiology, Faculty of Medicine, UNSW Sydney.

Neurologist, Neurology Department, Royal Prince Alfred Hospital.

The George Institute for Global Health at Peking University Health Science Center, Beijing, P.R.China.

The George Institute for Global Health, Sydney, Australia.

Huy Thang-Nguyen

Neurologist, The People's Hospital 115, Neurology, Ho Chi Min City, Vietnam John Gommans

Physician, Hawke's Bay Hospital, Hastings, New Zealand

Qilong Yi

Associate Professor, Canadian Blood Services and University of Toronto, Canada

\section{Contributors}

GJH was Chief Investigator A of the NHMRC Project Grant 1059094, Co-Chair of the steering committee, Chair of the trial coordinating committee, involved in the design of the trial, recruited and followed-up patients enrolled at his hospital site, adjudicated all adverse and serious adverse events in the trial (blind to treatment allocation), and wrote the first and final versions of the manuscript.

MLH was Chief Investigator B of the NHMRC Project Grant 1059094, Co-Chair of the steering committee, involved in the trial design, and advised on management of the trial.

OPA was Chief Investigator C of the NHMRC Project Grant 1059094, participated in the steering committee, involved in the trial design, and advised on the management of depression within the trial.

LF was Chief Investigator D of the NHMRC Project Grant 1059094, participated in the steering committee, and was involved in the trial design.

GEM was Chief Investigator E of the NHMRC Project Grant 1059094, participated in the steering committee as co-CI of the FOCUS trial, and was involved in the trial design.

MSD was Chief Investigator F of the NHMRC Project Grant 1059094, participated in the steering committee as co-CI of the FOCUS trial, was involved in the trial design, and advised regularly about trial management.

C E-B was Chief Investigator G of the NHMRC Project Grant 1059094 and participated in the steering committee.

AHF was Chief Investigator H of the NHMRC Project Grant 1059094, participated in the steering committee, and advised on the management of depression in the trial. 
LB was Chief Investigator I of the NHMRC Project Grant 1059094, participated in the steering committee, was involved in the trial design, advised on the statistical analysis plan and independently analysed the trial data and verified the final analysis.

SJ was Chief Investigator E of the NHMRC Project Grant 1059094 from 2014-2018, and participated in the steering committee.

TL was Chief Investigator E of the NHMRC Project Grant 1059094 in 2019.

VM (deceased December 2014) was an Associate Investigator of the NHMRC Project Grant 1059094, participated in the steering committee as CI of the EFFECTS trial, and was involved in the trial design.

EL participated in the steering committee as CI of the EFFECTS trial, and was involved in the trial design.

CSA was an Associate Investigator of the NHMRC Project Grant 1059094, participated in the steering committee, was involved in the trial design, and provided trial strategic advice.

JG was national coordinator for New Zealand, the Principal Investigator responsible for recruitment and follow-up of patients enrolled at his hospital site, and commented on the draft manuscript.

H T-N was national coordinator for Vietnam, and the Principal Investigator responsible for recruitment and follow-up of patients enrolled at his hospital site.

QY undertook the statistical analysis of the trial data for the Data Monitoring Committee meetings and for the final results.

The contributions other members of the AFFINITY trial collaboration are listed in the appendix (pp1-7).

\section{Declaration of interests}

Prof. Hankey reports grants from the National Health \& Medical Research Council of Australia, Vetenskapsrådet (The Swedish Research Council), and United Kingdom National Institute for Health Research Technology (NIHR), during the conduct of the study; and personal fees from American Heart Association, outside the submitted work.

Prof. Hackett reports grants from National Health and Medical Research Council during the conduct of the study.

Prof. Etherton-Beer reports grants from National Health and Medical Research Council (NHMRC) of Australia, during the conduct of the study.

Prof. Billot reports grants from NHMRC during the conduct of the study.

Dr. Lung reports grants from Australian National Health and Medical Research Council, during the conduct of the study. 
Prof. Anderson reports grants from National Health and Medical Research Council (NHMRC) of Australia, grants from Takeda, personal fees from Takeda, outside the submitted work.

Profs Almeida, Flicker, Mead, Dennis, Ford, Billot, Jan, Lundström, Thang-Nguyen, Gommans, and Yi report nothing to disclose.

\section{Data sharing}

The trial protocol and statistical analysis plan have been published. ${ }^{7,8}$ A fully anonymised trial dataset with individual patient data and a data dictionary will be available to other researchers after the publication of the full trial results from the final follow-up at 12 months. Written proposals and requests are to be directed to Graeme Hankey (Co-Chief Investigator). Proposals will be assessed by the AFFINITY trial Steering Committee and a data sharing agreement established if, and before, any data are to be shared.

\section{Acknowledgements}

The AFFINITY trial was funded by the Australian National Health and Medical Research Council (NHMRC) Project Grant 1059094. The minimisation algorithm was provided by 'The Stroke Research Group, Division of Clinical Neuroscience, The University of Edinburgh. The manufacture, storage and distribution of the trial medication was undertaken by Pharmaceutical Packaging Professionals Pty Ltd (Trading as PCI Pharma Services), an Australian Therapeutic Goods Administration-licenced Good Manufacturing Practices facility. We are grateful to all patients and their families who participated in AFFINITY, and the nursing staff who assisted at collaborating sites for contributing to this research endeavour. 
Table 1. Patient characteristics at randomization by allocated treatment

\begin{tabular}{|c|c|c|}
\hline & $\begin{array}{c}\text { Fluoxetine } \\
(n=642)\end{array}$ & $\begin{array}{l}\text { Placebo } \\
(n=638)\end{array}$ \\
\hline \multicolumn{3}{|l|}{ Sex } \\
\hline Women & $231(36 \%)$ & $245(38 \%)$ \\
\hline Men & $411(64 \%)$ & $393(62 \%)$ \\
\hline \multicolumn{3}{|l|}{ Age } \\
\hline Age $\leq 70$ years & $450(70 \%)$ & $432(68 \%)$ \\
\hline Age $>70$ years & $192(30 \%)$ & $206(32 \%)$ \\
\hline Mean age, years & $63.5(12.5)$ & $64.6(12.2)$ \\
\hline \multicolumn{3}{|l|}{ Ethnicity } \\
\hline Asian & $356(55 \%)$ & $371(58 \%)$ \\
\hline White & $267(42 \%)$ & $255(40 \%)$ \\
\hline Other & $19(3 \%)$ & $12(2 \%)$ \\
\hline \multicolumn{3}{|l|}{ Marital status } \\
\hline Married & $463(72 \%)$ & $463(73 \%)$ \\
\hline Partner & $29(4 \%)$ & $19(3 \%)$ \\
\hline Divorced or separated & $37(6 \%)$ & $46(7 \%)$ \\
\hline Widowed & $61(9 \%)$ & $70(11 \%)$ \\
\hline Single & $52(8 \%)$ & $38(6 \%)$ \\
\hline Other & $0(0 \%)$ & $2(0 \%)$ \\
\hline \multicolumn{3}{|l|}{ Living arrangements } \\
\hline Living with someone else & $564(88 \%)$ & $556(87 \%)$ \\
\hline Living alone & $78(12 \%)$ & $78(12 \%)$ \\
\hline Living in an institution & $0(0 \%)$ & $2(0 \%)$ \\
\hline Other & $0(0 \%)$ & $2(0 \%)$ \\
\hline \multicolumn{3}{|l|}{ Employment status } \\
\hline Full-time employment & $206(32 \%)$ & $180(28 \%)$ \\
\hline Part-time employment & $77(12 \%)$ & $68(11 \%)$ \\
\hline Retired & $315(49 \%)$ & $363(57 \%)$ \\
\hline Unemployed or disabled & $24(4 \%)$ & $14(2 \%)$ \\
\hline Other & $20(3 \%)$ & $13(2 \%)$ \\
\hline Independent before stroke & $634(99 \%)$ & $630(99 \%)$ \\
\hline \multicolumn{3}{|l|}{ Previous medical history } \\
\hline Coronary Heart Disease & $58(9 \%)$ & $57(9 \%)$ \\
\hline Ischaemic stroke or TIA & $77(12 \%)$ & $84(13 \%)$ \\
\hline Diabetes & $143(23 \%)$ & $147(23 \%)$ \\
\hline Hyponatraemia & $1(0 \%)$ & $3(0 \%)$ \\
\hline Intracranial bleed & $11(2 \%)$ & $8(1 \%)$ \\
\hline Upper gastrointestinal bleed & $11(2 \%)$ & $15(2 \%)$ \\
\hline Bone fractures & $71(11 \%)$ & $74(12 \%)$ \\
\hline Depression & $30(5 \%)$ & $20(3 \%)$ \\
\hline \multicolumn{3}{|l|}{ Stroke diagnosis } \\
\hline Non-stroke (final diagnosis) & $3(0 \%)$ & $1(0 \%)$ \\
\hline Ischaemic stroke & $549(86 \%)$ & $542(85 \%)$ \\
\hline
\end{tabular}




\begin{tabular}{|c|c|c|}
\hline Intracerebral haemorrhage & $90(14 \%)$ & $95(15 \%)$ \\
\hline \multicolumn{3}{|l|}{ OCSP classification of Ischaemic stroke } \\
\hline Total anterior circulation infarct & $47(9 \%)$ & $50(9 \%)$ \\
\hline Partial anterior circulation infarct & $271(49 \%)$ & $283(52 \%)$ \\
\hline Lacunar infarct & $115(21 \%)$ & $105(19 \%)$ \\
\hline Posterior circulation infarct & $114(21 \%)$ & $103(19 \%)$ \\
\hline Uncertain & $2(0 \%)$ & $1(0 \%)$ \\
\hline \multicolumn{3}{|l|}{$\begin{array}{l}\text { Causes of ischaemic stroke (modified } \\
\text { TOAST classification) }\end{array}$} \\
\hline Large artery disease & $123(22 \%)$ & $134(25 \%)$ \\
\hline Small vessel disease & $261(47 \%)$ & $250(46 \%)$ \\
\hline Embolism from the heart & $95(17 \%)$ & $93(17 \%)$ \\
\hline Another cause & $9(2 \%)$ & $8(1 \%)$ \\
\hline Unknown or uncertain cause & $61(11 \%)$ & $57(10 \%)$ \\
\hline \multicolumn{3}{|l|}{ Predictive variables } \\
\hline Able to walk at time of randomisation & $282(44 \%)$ & $279(44 \%)$ \\
\hline Able to lift both arms off bed & $443(69 \%)$ & $431(68 \%)$ \\
\hline Able to talk and not confused & $554(86 \%)$ & $557(87 \%)$ \\
\hline \multicolumn{3}{|l|}{$\begin{array}{l}\text { Predicted 6-month outcome based on } \\
\text { SSV }\end{array}$} \\
\hline $\begin{array}{l}\text { Probability of being alive and } \\
\text { independent }\end{array}$ & $\begin{array}{c}0.57 \\
(0.26-0.87) \\
\end{array}$ & $\begin{array}{c}0.55 \\
(0.24-0.87) \\
\end{array}$ \\
\hline 0.00 to $\leq 0.15$ & $100(15 \%)$ & $103(16 \%)$ \\
\hline 0.15 to 1.00 & $542(84 \%)$ & $535(84 \%)$ \\
\hline \multicolumn{3}{|l|}{ Neurological deficits } \\
\hline NIHSS & $6(3-9)$ & $6(3-9)$ \\
\hline Presence of a motor deficit & $557(87 \%)$ & $548(86 \%)$ \\
\hline Presence of aphasia & $129(20 \%)$ & $121(19 \%)$ \\
\hline \multicolumn{3}{|l|}{ Depression at baseline } \\
\hline $\begin{array}{l}\text { Current diagnosis of depression (patient } \\
\text { or proxy reported) }\end{array}$ & $15(2 \%)$ & $17(3 \%)$ \\
\hline Taking a non-SSRI antidepressant & $5(1 \%)$ & $5(1 \%)$ \\
\hline \multicolumn{3}{|l|}{ Current mood } \\
\hline PHQ-9, median (IQR) & $4(1-7)$ & $4(2-7)$ \\
\hline $0-14$ & $601(98 \%)$ & $596(98 \%)$ \\
\hline$\geq 15$ & $12(2 \%)$ & $11(2 \%)$ \\
\hline \multicolumn{3}{|l|}{$\begin{array}{l}\text { Delay (days) since stroke onset at } \\
\text { randomisation }\end{array}$} \\
\hline Mean delay & $6.1(3 \%)$ & $6.3(3 \%)$ \\
\hline 2-8 days & $486(76 \%)$ & $479(75 \%)$ \\
\hline 9-15 days & $156(24 \%)$ & $159(25 \%)$ \\
\hline \multicolumn{3}{|l|}{ Consent } \\
\hline Patient consented & $345(54 \%)$ & $328(51 \%)$ \\
\hline Person responsible consented & $284(44 \%)$ & $295(46 \%)$ \\
\hline Proxy consented & $3(0 \%)$ & $1(0 \%)$ \\
\hline Waiver acknowledgement & $10(2 \%)$ & $14(2 \%)$ \\
\hline
\end{tabular}


Data are $\mathrm{n}(\%)$, mean (SD [standard deviation]), or median (IQR [interquartile range]) TIA: transient ischaemic attack

OCSP $=$ Oxfordshire Community Stroke Project

TOAST $=$ modified Trial of ORG 10172 in acute stroke treatment criteria

$\mathrm{SSV}=$ Six simple variables the predict functional outcome, as measured by the mRS, after stroke (age, living alone before the stroke, independent in activities of daily living before the stroke, and able to talk, lift both arms off the bed, and walk unassisted at the time of randomisation). ${ }^{15}$

NIHSS = National Institutes of Health Research Stroke Scale

SSRI: selective serotonin reuptake inhibitor

PHQ-9 = Patient Health Questionnaire 9 items (higher scores indicate more depressive symptoms). ${ }^{17}$ 
Table 2. Secondary outcomes at six months by allocated treatment

\begin{tabular}{|l|c|c|c|c|c|}
\hline \multicolumn{2}{|c|}{ Fluoxetine (n=642) } & \multicolumn{2}{|c|}{ Placebo (n=638) } & P value \\
\hline $\begin{array}{l}\text { New depression } \\
\text { N / N (\%) }\end{array}$ & $33(5.1 \%)$ & & $46(7.2 \%)$ & & 0.13 \\
\hline Mood (PHQ-9) & 2.0 & $(1.0-5.0)$ & 2.0 & $(1.0-5.0)$ & 0.42 \\
\hline $\begin{array}{l}\text { PHQ-9 } \geq 15 \\
\text { N / N\% }\end{array}$ & $4(0.7 \%)$ & & $6(1.0 \%)$ & & 0.75 \\
\hline Cognition (TICSm) & 24.0 & $(20.0-27.0)$ & 24.0 & $(19.0-27.0)$ & 0.62 \\
\hline $\begin{array}{l}\text { Stroke Impact Scale } \\
\text { SIS) domains }\end{array}$ & & & & & \\
\hline -Strength & 75.0 & $(56.3-93.8)$ & 75.0 & $(56.3-93.8)$ & 0.26 \\
\hline -Hand ability & 85.0 & $(55.0-100.0)$ & 85.0 & $(55.0-100.0)$ & 0.39 \\
\hline -Mobility & 91.7 & $(69.4-100.0)$ & 88.9 & $(66.7-97.2)$ & 0.08 \\
\hline -Motor† & 83.5 & $(63.5-94.2)$ & 82.4 & $(60.4-93.2)$ & 0.28 \\
\hline -Daily Activities & 90.0 & $(72.5-100.0)$ & 90.0 & $(70.0-97.5)$ & 0.25 \\
\hline -Physical function† & 85.5 & $(66.2-94.9)$ & 83.8 & $(63.4-93.8)$ & 0.24 \\
\hline -Memory & 89.3 & $(78.6-100.0)$ & 89.3 & $(75.0-100.0)$ & 0.28 \\
\hline -Communication & 98.2 & $(89.3-100.0)$ & 96.4 & $(85.7-100.0)$ & 0.61 \\
\hline -Mood/Emotions^ & 80.6 & $(66.7-88.9)$ & 77.8 & $(66.7-86.1)$ & 0.003 \\
\hline -Participation & 81.3 & $(59.4-96.9)$ & 75.0 & $(56.3-96.9)$ & 0.48 \\
\hline -Recovery (VAS) & 80.0 & $(60.0-90.0)$ & 80.0 & $(60.0-90.0)$ & 0.90 \\
\hline Vitality (SF-36) & 70.0 & $(55.0-80.0)$ & 70.0 & $(55.0-80.0)$ & 0.36 \\
\hline EQ5D-5L & 0.81 & $0.63-1.00$ & 0.78 & $0.58-0.93$ & 0.08 \\
\hline
\end{tabular}

Data were only available for those who survived and who completed sufficient questions to derive a score. The number of patients with missing scores were similar in the two treatment groups.

Data are median (IQR).

PHQ-9: Patient Health Questionnaire 9 items (higher score indicates more depressive symptoms)

TICSm: Telephone Interview for Cognitive Status

SIS: Stroke Impact Scale (where higher scores are better).

$\dagger$ Mean of the Strength, Hand ability, and Mobility domains.

$\$$ Mean of the Strength, Hand ability, Mobility, and Daily activities domains.

${ }^{\wedge}$ Mood/Emotions domain of the SIS: Nine questions about "how you feel, changes in your mood, and your ability to control your emotions, since your stroke." (where higher scores are better)

VAS: visual analogue scale.

SF-36: 36 item short form questionnaire (higher scores indicate less disability) 
EQ5D-5L: EuroQoL - 5 Dimensions (Mobility, Personal Care, Usual Activities, Pain/Discomfort, Anxiety/Depression) - 5 Levels (where 1 indicates the best health imaginable, and -0.676 indicates the worst health imaginable). 
Table 3. Adverse events at 6 months by allocated treatment group

\begin{tabular}{|c|c|c|c|c|}
\hline & $\begin{array}{c}\text { Fluoxetine } \\
(n=642)\end{array}$ & $\begin{array}{l}\text { Placebo } \\
(n=638)\end{array}$ & Difference $(95 \% \text { CI })^{*}$ & $\begin{array}{c}P- \\
\text { value }\end{array}$ \\
\hline Death & $15(2.34 \%)$ & $15(2.35 \%)$ & $-0.01 \%(-1.64$ to 1.67$)$ & 1.00 \\
\hline Any stroke & $18(2.80 \%)$ & $26(4.08 \%)$ & $-1.27 \%(-3.27$ to 0.72$)$ & 0.22 \\
\hline \multicolumn{5}{|l|}{ All thrombotic events } \\
\hline Ischaemic stroke & $11(1.71 \%)$ & $21(3.29 \%)$ & $-1.58 \%(-3.29$ to 0.13$)$ & 0.08 \\
\hline Acute coronary events & $1(0.16 \%)$ & $2(0.31 \%)$ & $-0.16 \%(-0.69$ to 0.37$)$ & 0.62 \\
\hline \multicolumn{5}{|l|}{ All bleeding events } \\
\hline Haemorrhagic stroke & $3(0.47 \%)$ & $1(0.16 \%)$ & $0.31 \%(-0.30$ to 0.92$)$ & 0.62 \\
\hline Upper gastrointestinal bleed & $1(0.16 \%)$ & $1(0.16 \%)$ & $0.00 \%(-0.43$ to 0.43$)$ & 1.00 \\
\hline Epileptic seizures & $10(1.56 \%)$ & $2(0.31 \%)$ & $1.24 \%(0.19$ to 2.30$)$ & 0.04 \\
\hline Fall with injury & $20(3.12 \%)$ & $7(1.10 \%)$ & $2.02 \%(0.45$ to 3.59$)$ & 0.02 \\
\hline New bone fracture & $19(2.96 \%)$ & $6(0.94 \%)$ & $2.02 \%(0.51$ to 3.53$)$ & 0.01 \\
\hline Hyponatraemia $<125 \mathrm{mmol} / 1$ & $3(0.47 \%)$ & $2(0.31 \%)$ & $0.15 \%(-0.53$ to 0.84$)$ & 1.00 \\
\hline Hyperglycaemia & $0(0 \%)$ & $0(0 \%)$ & $0 \%$ & \\
\hline Symptomatic hypoglycaemia & $0(0 \%)$ & $0(0 \%)$ & $0 \%$ & \\
\hline New depression & $33(5.14 \%)$ & $46(7.21 \%)$ & $-2.07 \%(-4.71$ to 0.57$)$ & 0.13 \\
\hline New antidepressant & $30(4.67 \%)$ & $43(6.74 \%)$ & $-2.07 \%(-4.61$ to 0.47$)$ & 0.12 \\
\hline Attempted or actual suicide & $0(0 \%)$ & $2(0.31 \%)$ & $-0.16 \%(-0.75$ to 0.12$)$ & 0.25 \\
\hline Other adverse event & $62(9.66 \%)$ & $68(10.66 \%)$ & $-1.00 \%(-4.31$ to 2.31$)$ & 0.56 \\
\hline
\end{tabular}

Data are n (\%), unless otherwise stated

* Risk differences and their 95\%confidence intervals were calculated in SAS by means of the FREQ procedure.

https://documentation.sas.com/?docsetId=procstat\&docsetTarget=procstat_freq_detail $\underline{\text { s54.htm \&docsetVersion }=9.4 \& \text { locale }=\text { en }}$ (accessed March 18, 2020). The confidence intervals around the risk differences are Wald intervals based on the normal approximation. 


\section{Supplementary Appendix}

\section{Contributors to the AFFINITY trial collaboration}

\section{Co-Chief Investigators}

Graeme J. Hankey

Maree L. Hackett

\section{Trial Coordinating Centre, Perth, Western Australia}

Sarah Barrett, Anne Claxton, Julia O’Dea, Michelle Tang, Clare Williams

Administration Support

Shenae Peterson, Christie Drummond

\section{HORUS Vietnam Regional Coordinating Center, Ho Chi Minh City, Vietnam.}

Hong Ha Uyen Pharm., Le Thi My Linh Pharm., Ngo Thi Bich Tram Pharm., Mai Bao Yen, Han Thanh Huyen, Truong Quynh Nhu Pharm., Nguyen Thi Huong, Ngo Thanh Hai Pharm., Nguyen Thi Binh Thuan, Ha Thi Kieu Oanh Pharm., Nguyen Le Huyen Trang Pharm.

\section{Steering Committee}

Graeme J. Hankey, MD, FRACP, FRCP (chair)

Professor of Neurology, Medical School, Faculty of Health and Medical Sciences, The University of Western Australia, Perth, Western Australia;

Neurologist, Department of Neurology Sir Charles Gairdner Hospital, Perth, Australia

Maree L. Hackett, PhD

Professor, The George Institute for Global Health, Faculty of Medicine,

University of New South Wales Sydney;

Professor of Epidemiology, The University of Central Lancashire, United Kingdom.

Osvaldo P. Almeida

Professor of Geriatric Psychiatry, Medical School, Faculty of Health and Medical Sciences, The University of Western Australia, Perth, Western Australia

Leon Flicker

Professor of Geriatric Medicine, Medical School, Faculty of Health and Medical Sciences, The University of Western Australia, Perth, Western Australia

Geriatrician, Royal Perth Hospital, Perth, Australia

Gillian E. Mead

Professor of Stroke and Elderly Care Medicine, University of Edinburgh, Royal Infirmary, Edinburgh

Martin S. Dennis 
Professor of Stroke Medicine, University of Edinburgh, Royal Infirmary, Edinburgh Christopher Etherton-Beer

Associate Professor, The University of Western Australia, Perth, Western Australia Andrew H. Ford

Associate Professor, The University of Western Australia, Perth, Western Australia Laurent Billot

Director, Statistics Division, The George Institute for Global Health, New South Wales, Australia

Associate Professor, Faculty of Medicine, UNSW, Sydney

Stephen Jan

Professor of Health Economics, The George Institute for Global Health, Faculty of Medicine, University of New South Wales, Sydney, Australia

Veronica Murray (deceased December 2014)

Danderyd Hospital, Stockholm, Sweden

Erik Lundström, $\mathrm{PhD}$,

Associate Professor, Department of Neuroscience, Neurology, Uppsala University, Uppsala, Sweden.

Craig S. Anderson MD PhD FRACP

Professor of Neurology and Epidemiology, Faculty of Medicine, UNSW Sydney. Neurologist, Neurology Department, Royal Prince Alfred Hospital. The George Institute for Global Health at Peking University Health Science Center, Beijing, P.R.China.

The George Institute for Global Health, Sydney, Australia.

\section{Independent Data Monitoring Committee}

Rob Herbert (Chair)

Professor, Neuroscience Research Australia, Sydney, Australia

Gregory Carter

Professor, Centre for Translational Neuroscience \& Mental Health, University of Newcastle, New South Wales, Australia

Geoffrey A. Donnan

Professor, Melbourne Brain Centre, Royal Melbourne Hospital, Victoria, Australia 


\section{National coordinators}

Vietnam

Huy Thang-Nguyen, The People's Hospital 115, Neurology, Ho Chi Min City, Vietnam

Australia

Graeme J. Hankey, Medical School, The University of Western Australia

New Zealand

John Gommans, Hawke's Bay Hospital, Hastings, New Zealand

\section{Unblinded Statisticians}

Qilong Yi, Associate Professor, Canadian Blood Services and University of Toronto, Canada

Qiang Li, The George Institute for Global Health, Sydney

Severine Bompoint, The George Institute for Global Health, Sydney

\section{Health Economist}

Thomas Lung, The George Institute for Global Health, Sydney

\section{Human Research Ethics Committee (HREC) Liaison lead investigators}

Australia

Richard I. Lindley (New South Wales, Queensland, Victoria)

Peter New (Victoria: Caulfield Hospital)

Andrew Lee (South Australia)

Graeme J. Hankey (Western Australia)

New Zealand

John Gommans

\section{Minimisation Algorithms}

The Stroke Research Group, Division of Clinical Neuroscience, The University of Edinburgh.

\section{Investigational Medicinal Product}

Manufactured by Siegfried Malta Ltd (fluoxetine, certificate MT/008HM/2017) and Pharmaceutical packaging professionals (PPP) Pty Ltd (placebo) 
Packaged by Amneal Pharmaceuticals Pty Ltd (Australian Therapeutic Goods Administration [TGA] licence holder).

Re-packaged (as patient kits, two bottles, each containing 110 capsules), labelled (with trialspecific treatment codes, fluoxetine or placebo), and distributed by PPP Pty Ltd.

Export to New Zealand and Vietnam approved by the Australian Government Department of Health TGA (Approval Ref No: EX17/336513).

\section{Funding}

National Health and Medical Research Council, Australia

\section{Registration}

Australian New Zealand Clinical Trial Registry number: ACTRN12611000774921

\section{Sponsors}

Royal Perth Hospital and Sir Charles Gairdner Hospital, Perth, Western Australia

\section{Participating centres and number of patients randomised in each treatment group.}

The participating centres are grouped by the highest to lowest recruiting country, followed by highest to lowest recruiting site. If centres have equal recruiting numbers, the sites are listed by alphabetical order. We have listed each centre by site number and name with the total number of patients recruited in [n], followed by names of the local principal investigator(s), and other significant contributors in that centre.

\section{Vietnam [706 patients recruited]}

Nguyen Tri Phuong Hospital [179] ((Tran Trung Thanh (PI), Le Tran Truc Mai Loan, Kieu Le Vu Thuy, Nguyen Van Sang, Nguyen Anh Diem Thuy, Dang Nhat Tam);

The Peoples Hospital 115 [110] (Nguyen Huy Thang (PI), Truong Le Tuan Anh, Dam Thi Cam Linh, Bui Thi Quynh Chau, Ngo Thi Kim Trinh, Pham Nguyen Thanh Thai, Luong Van Dong, Doan Van Tan, Ma Hoa Hung, Pham Nguyen Binh, Phan Dang Loc, Dao Thi Thanh Nha, Nguyen Thi Bich Huong, Le Thi Cam Linh, Do Minh Chi, Huynh Quoc Huy, Nguyen Quoc Trung, Nguyen Thanh Thai An);

Nghe An General Friendship Hospital [100] (Duong Dinh Chinh (PI), Kieu Van Duong, Le Na, Nguyen Ngoc Hoa, Le Van Binh, Nguyen Thanh Long); 
Gia Dinh Peoples Hospital [79] (Vo Van Tan (PI), Bui Ngoc Tram, Hoang Thi To Uyên, Nguyen Thi Bich Hien, Nguyen Thi Thu Ha, Lam Thuy Nga, Le Kim Khanh, Trinh Thanh Phuong);

Thanh Hoa General Hospital [63] (Nguyen Hoanh Sam (PI), Le Hong Ninh, Nguyen Truong Giang, Doan Thi Bich, Pham Phuoc Sung, Luong Huu Duong, Mai Van Ha);

Bach Mai Hospital [61] (Nguyen Van Chi (PI), Nguyen Doan Phuong (PI), Mai Duy Ton, Dao Viet Phuong, Nguyen Tien Dung, Khuong Quoc Dai, Vuong Xuan Trung, Vu Tuong Lan, Ngo Duc Ngoc);

Central Military Hospital 108 [42] (Nguyen Hoang Ngoc (PI), Nguyen Van Tuyen, Le Dinh Toan, Dinh Hai Ha, Pham Van Cuong, Thach Thi Ngoc Khanh, Nguyen Hai Linh, Nguyen Thi Loan);

Cho Ray Hospital [33] (Nguyen Anh Tai (PI), Le Van Tuan, Truong Van Luyen, Bui Chau Tue, Tran Van Nhat, Huynh Xuan Ngọc, Dinh Van Lap, Pham Gia An, Nguyen Tuong Vy);

University Medical Center [22] (Nguyen Ba Thang (PI), Thai Huy, Pham Thi Ngoc Quyen, Dao Duy Khoa, Pham Nguyen Bao Quoc, Dang Thi Huyen Thuong, Dinh Huynh To Huong, Tong Mai Trang, Vu Thi Thuy, Le Tri Si, Tran Ngoc Tai, Tran Hoai Phuong);

National Geriatric Hospital [17] (Nguyen Thanh Binh (PI), Ngo Trong Toan, Le Chung Thuy, Nguyen Anh Dung, Nguyen Thanh Binh, Do Phuong Vinh).

\section{Australia [532 patients recruited]}

Sir Charles Gairdner Hospital, WA, [93] (David Blacker (PI), Graeme Hankey, Anne Claxton, Lindsey Bunce, Ai Ling Tan);

Fiona Stanley Hospital, WA, [80] (Darshan Ghia (PI), Gillian Edmonds, Nicole O’Loughlin, Megan Ewing, Kerri-Ann Whittaker, Lorralee Deane);

Royal Perth Hospital, WA, [78] (Darshan Ghia (PI), Graeme Hankey, Anne Claxton);

Calvary Health Care Bruce, ACT, [34] (Yash Gawarikar (PI), Brett Jones, Maria Lopez, Koushik Nagesh, Emma Siracusa);

Royal Melbourne Hospital, VIC, [30] (Stephen Davis (PI), Amy McDonald, Jess Tsoleridis, Rachael McCoy, David Jackson, Gab Silver);

St John of God Hospital Midland, WA, [25] (Tim Bates (PI), Amanda Boudville, Lynda Southwell);

Liverpool Hospital, NSW, [22] (Dennis Cordato (PI), Alan J McDougall, Cecilia CappelenSmith, Zeljka Calic, Shabeel Askar, Qi Cheng, Raymond Kumar);

Redcliffe Hospital, QLD, [18] (Richard Geraghty (PI), Maree Duroux, Megan Ratcliffe, Samantha Shone, Cassandra McLennan);

Ballarat Base Hospital, VIC, [16] (Ramesh Sahathevan (PI), Casey Hair); 
Campbelltown \& Camden Hospitals, NSW, [13] (Stanley Levy (PI), Beverley Macdonald, Benjamin Nham, Louise Rigney, Dev Nathani, Sumana Gopinath, Vishal Patel, Abul Mamun, Benjamin Trewin, Chun Phua, Ho Choong);

Belmont Hospital, NSW, [11] (Lauren Tarrant (PI) Kerry Boyle, Luisa Hewitt, Monique Hourn, Amanda Masterson, Kim Oakley, Karen Ruddell, Colette Sanctuary, Kimberley Veitch, Jenni White);

Blacktown Hospital, NSW, [11] (Richard Lindley (PI), Camelia Burdusel, Lina Lee, Gary Cheuk);

Shoalhaven District Memorial Hospital, NSW, [11] (Jeremy Christley (PI), Tabitha Hartwell, Craig Davenport, Kate Hickey, Rosanna Robertson, Michelle Carr);

Kingston Hospital, VIC, [10] (Peter New (PI) Sam Akbari, Hannah Coyle, Megan O’Neill, Cameron Redpath, Caroline Roberts, Marjan Tabesh, Toni Withiel);

Osborne Park Hospital, WA, [10] ( Kapila Abeysuriya (PI), Andrew Granger (PI), Angela Abraham, Chermaine Chua, Dung Do Nguyen, Vathani Surendran, Melissa Daines, David Shivlal, Mudassar Latif, Noreen Mughal, Patricia Morgan);

Royal North Shore Hospital, NSW, [10] (Martin Krause (PI), Miriam Priglinger, Ehsan Esmaili Shandiz, Susan Day);

Swan District Hospital, WA, [9] (Tim Bates (PI), Lay Kho);

Rankin Park Rehab Unit, NSW, [8]( Michael Pollack (PI) Judith Dunne, Helen Baines, Merridie Rees, Jenni White, Monique Hourn, Luisa Hewitt, Kimberley Veitch, Aicuratiya Withanage, Colette Sanctuary);

Royal Prince Alfred Hospital, NSW, [8] (Craig Anderson (PI), Candice Delcourt, Cheryl Carcel, Alejandra Malavera, Amy Kunchok, Elizabeth Ray);

Calvary Mater Newcastle, NSW, [6] (Elizabeth Pepper (PI) Emily Duckett, Jenni White, Kimberley Veitch, Luisa Hewitt, Monique Hourn, Kerry Boyle, Sally Ormond, Colette Sanctuary);

Lyell McEwin, SA, [6] (Andrew Moey (PI 2017-2019), Timothy Kleinig (PI 2016-2017), Vanessa Maxwell, Chantal Baldwin, Wilson Vallat, Deborah Field);

St Vincent's Hospital, NSW, [5] (Romesh Markus (PI), Kirsty Page, Danielle Wheelwright, Sam Bolitho, Steven Faux, Fix Sangvatanakul, Alexis Brown, Susan Walker, Jennifer Massey);

John Hunter Hospital, NSW, [4] (Michael Pollack (PI) (Jenni White (PI), Kimberley Veitch, Hillary Hayes, Luisa Hewitt, Monique Hourn, Colette Sanctuary);

Prince of Wales, NSW, [4] (Pesi Katrak (PI) (Annie Winker PI), Alessandro Zagami, Alanah Bailey, Sarah Mccormack, Andrew Murray, Mark Rollason, Christopher Taylor); 
Bankstown Hospital, NSW, [3] (Fintan O’Rourke PI), Ye Min Kuang, Heike Burnet, Yvonne Liu, Qi Cheng, Aileen Wu);

Caulfield Hospital, VIC, [2] (Peter New (PI), Sam Akbari, Hannah Coyle, Megan O’Neill, Diana Ramirez);

Footscray \& The Sunshine Hospitals, VIC, [2] (Tissa Wijeratne (PI), Sherisse Celestino, Essie Low, Cynthia Chen, Jennifer Bergqvist);

Manly Hospital, NSW, [2] (Andrew Evans (PI) Queenie Leung);

Wagga Wagga Base Hospital, NSW, [1] (Martin Jude (PI), Rachael McQueen, Katherine Mohr, Latitia Kernaghan);

Flinders Medical Centre, SA, (Andrew Lee (PI), Paul Stockle, Boon Loong Tan, Sara Laubscher);

Australian participating States Abbreviation Key:

NSW $=$ New South Wales

QLD = Queensland

SA $=$ South Australia

VIC $=$ Victoria

WA $=$ Western Australia

\section{New Zealand [42 patients recruited]}

Hawke’s Bay Hospital [14] (John Gommans (PI), Diana Schmid, Melissa Spooner); Taranaki Base Hospital [14] (Bhavesh Lallu (PI), Bronwen Pepperell, John Chalissery); Rotorua Hospital [8] (Karim Mahawish (PI), Susan DeCaigney, Paula Broughton, Karen Knight, Veronica Duque);

Wellington Hospital [6] (Harry McNaughton (PI), Jeremy Lanford, Vivian Fu, Lai-Kin Wong); 
Supplementary table 1.

Methods of patient follow-up

\begin{tabular}{|c|c|c|c|}
\hline Method of Follow-up & $\begin{array}{c}\text { Fluoxetine } \\
(n=642)\end{array}$ & $\begin{array}{l}\text { Placebo } \\
(n=638)\end{array}$ & $\begin{array}{c}\text { p-values } \\
(\mathrm{A}, \mathrm{B})\end{array}$ \\
\hline \multicolumn{4}{|l|}{ Day 28} \\
\hline Outpatient Clinic & 345 & 356 & \\
\hline Telephone & 195 & 212 & \\
\hline Hospital & 76 & 57 & \\
\hline Other (e.g. email, home visit) & 20 & 11 & \\
\hline Missing & 2 & 1 & \\
\hline Total submitted & 638 & 637 & $0.0928\left(0.1290^{\mathrm{B}}\right)$ \\
\hline \multicolumn{4}{|l|}{ Day 90} \\
\hline Outpatient Clinic & 357 & 360 & \\
\hline Telephone & 233 & 239 & \\
\hline Hospital & 14 & 6 & \\
\hline Other (e.g. email, home visit) & 21 & 26 & \\
\hline Missing & 4 & 0 & \\
\hline Total submitted & 629 & 631 & $0.2848\left(0.1020^{\mathrm{B}}\right)$ \\
\hline \multicolumn{4}{|l|}{ Day 180} \\
\hline Outpatient Clinic & 295 & 321 & \\
\hline Telephone & 300 & 287 & \\
\hline Other (e.g. email, home visit) & 15 & 12 & \\
\hline Missing & 2 & 1 & \\
\hline Total submitted & 612 & 621 & $0.4410\left(0.5879^{\mathrm{B}}\right)$ \\
\hline
\end{tabular}

A. P-values are based on the combined methods of follow-up (in-hospital assessment, outpatient clinic, telephone, home visit, and email).

B. P-values in brackets include missing data as another category. 
Supplementary table 2.

Adherence to trial medication in each treatment group and overall:

temporary and permanent cessation at each follow-up

\begin{tabular}{|c|c|c|c|c|}
\hline & $\begin{array}{c}\text { Fluoxetine } \\
\text { (n=642), } \\
\text { N (\%) }\end{array}$ & $\begin{array}{c}\text { Placebo } \\
\text { (638), } \\
\text { N (\%) } \\
\end{array}$ & $\begin{array}{c}\text { Total } \\
(\mathrm{N}=1280), \\
\mathrm{N}(\%) \\
\end{array}$ & $\begin{array}{c}\text { p-value } \\
\text { (chi-square } \\
\text { test) }\end{array}$ \\
\hline \multicolumn{5}{|l|}{$\begin{array}{l}\text { Temporary } \\
\text { cessation }\end{array}$} \\
\hline 0-1 month & $46 / 638(7.2)$ & $37 / 637(5.8)$ & $83 / 1275(6.5)$ & 0.3104 \\
\hline 1-3 month & $34 / 629(5.4)$ & 28/631(4.4) & $62 / 1260(4.9)$ & 0.4270 \\
\hline 3-6 month & 19/612(3.1) & 14/621(2.3) & $33 / 1233(2.7)$ & 0.3551 \\
\hline Total $^{*}$ & 87 & 71 & 158 & \\
\hline \multicolumn{5}{|l|}{$\begin{array}{l}\text { Permanent } \\
\text { cessation }\end{array}$} \\
\hline $0-1$ month & $47 / 638(7.4)$ & 46/637 (7.2) & 93/1275 (7.3) & 0.9205 \\
\hline 1-3 month & $34 / 629(5.4)$ & $30 / 631(4.8)$ & $64 / 1260(5.2)$ & 0.5987 \\
\hline 3-6 month & $27 / 612(4.4)$ & 24/621(3.9) & 50/1233(4.1) & 0.6296 \\
\hline Total & 108 & 100 & 208 & \\
\hline
\end{tabular}

* Some patients reported temporary cessation of trial drug on multiple occasions. 


\section{Supplementary table 3.}

Compliance with trial medication (actual dosing history compared to the prescribed drug regimen of once daily, 7 times per week) in each treatment group and overall.*

\begin{tabular}{|c|c|c|c|c|}
\hline $\begin{array}{l}\text { Time point } \\
\text { Average number of } \\
\text { times medication taken } \\
\text { weekly }\end{array}$ & $\begin{array}{c}\text { Fluoxetine } \\
\qquad(\mathrm{n}=642)\end{array}$ & $\begin{array}{c}\text { Placebo } \\
\text { (638) }\end{array}$ & $\begin{array}{c}\text { Total } \\
(\mathrm{N}=1280) \\
\mathrm{N}(\%)\end{array}$ & p-values \\
\hline 1 month & & & & 0.5703 \\
\hline 0 times per week & $26 / 630(4.1)$ & $25 / 631(4.0)$ & $51 / 1261(4.0)$ & \\
\hline 1-2 times per week & $16 / 630(2.5)$ & $9 / 631(1.4)$ & $25 / 1261(2.0)$ & \\
\hline 3-4 times per week & $8 / 630(1.3)$ & $10 / 631(1.6)$ & $18 / 1261(1.4)$ & \\
\hline 5-6 times per week & $24 / 630(3.8)$ & $17 / 631(2.7)$ & $41 / 1261(3.3)$ & \\
\hline 7 times per week & $556 / 630(88.3)$ & $570 / 631(90.3)$ & $1126 / 1261(89.3)$ & \\
\hline Missing & 8 & 6 & 14 & \\
\hline 3 months & & & & 0.9398 \\
\hline 0 times per week & $63 / 622(10.1)$ & 62 /625 (9.9) & $125 / 1247$ (10.0) & \\
\hline 1-2 times per week & $6 / 622(1.0)$ & $6 / 625(1.0)$ & $12 / 1247$ (1.0) & \\
\hline 3-4 times per week & $7 / 622(1.1)$ & $6 / 625(1.0)$ & $13 / 1247$ (1.0) & \\
\hline 5-6 times per week & $15 / 622(2.4)$ & $10 / 625(1.6)$ & $25 / 1247(2.0)$ & \\
\hline 7 times per week & $531 / 622(85.4)$ & $541 / 625$ (86.6 ) & $1072 / 1247$ (86.0 ) & \\
\hline Missing & 7 & 6 & 13 & \\
\hline 6 months & & & & 0.9190 \\
\hline 0 times per week & 72 /608 (11.8 ) & 76 /616 (12.3 ) & $148 / 1224$ (12.1) & \\
\hline 1-2 times per week & $5 / 608(0.8)$ & $5 / 616(0.8)$ & $10 / 1224(0.8)$ & \\
\hline 3-4 times per week & $6 / 608(1.0)$ & $8 / 616(1.3)$ & $14 / 1224(1.1)$ & \\
\hline 5-6 times per week & $10 / 608(1.6)$ & $15 / 616(2.4)$ & $25 / 1224(2.0)$ & \\
\hline 7 times per week & 515 /608 (84.7) & 512 /616 (83.1) & $1027 / 1224(83.9)$ & \\
\hline Missing & 4 & 5 & 9 & \\
\hline
\end{tabular}

\footnotetext{
* Proportions were calculated based on non-missing observations.
} 
Supplementary table 4.

Results from the ordinal and binary analyses of the mRS

\begin{tabular}{|l|c|c|}
\hline & Estimate $\mathbf{( 9 5 \% C I )}$ & p-value \\
\hline Unadjusted proportional odds model: & & \\
\hline Common odds ratio (OR) & $0.97(0.79$ to 1.18$)$ & 0.7354 \\
\hline Adjusted proportional odds model* & & \\
\hline Common OR & $0.94(0.76$ to 1.15$)$ & 0.5296 \\
\hline & & \\
\hline Unadjusted binary logistic regressions & & 0.7713 \\
\hline OR: $0 \quad$ vs $1-6$ & $0.95(0.66$ to 1.36$)$ & 0.8531 \\
\hline OR: $0-1$ vs $2-6$ & $1.02(0.82$ to 1.28$)$ & 0.2069 \\
\hline OR: $0-2$ vs $3-6$ & $0.86(0.67$ to 1.09$)$ & 0.8375 \\
\hline OR: $0-3$ vs $4-6$ & $1.04(0.72$ to 1.50$)$ & 0.9665 \\
\hline OR: $0-4$ vs $5-6$ & $0.99(0.53$ to 1.83$)$ & 0.9718 \\
\hline OR: $0-5$ vs 6 & $0.99(0.48$ to 2.04$)$ & \\
\hline
\end{tabular}

*The covariates used for the adjustment were the same as for the primary outcome analysis, that is, the analysis was adjusted for baseline minimization variables including delay since stroke onset computer-generated prediction of 6-month outcome, presence of a motor deficit and aphasia. 


\section{Supplementary table 5.}

Sequential per-protocol analysis of the 1256 patients with mRS data at 6 months.

\begin{tabular}{|c|c|c|c|c|c|c|c|c|}
\hline $\begin{array}{l}\text { Groups } \\
\text { cumulatively } \\
\text { excluded }\end{array}$ & $\begin{array}{l}\text { No. } \\
\text { meeting } \\
\text { each } \\
\text { exclusion } \\
\text { criteria }\end{array}$ & $\begin{array}{l}\text { Cumulative } \\
\text { no. removed } \\
\text { from } \\
\text { analysis }\end{array}$ & $\begin{array}{l}\text { No. } \\
\text { remaining } \\
\text { in } \\
\text { Fluoxetine } \\
\text { group }\end{array}$ & $\begin{array}{l}\text { No } \\
\text { remaining } \\
\text { in Placebo } \\
\text { group }\end{array}$ & $\begin{array}{l}\text { Common } \\
\text { OR for } \\
\text { mRS }\end{array}$ & $95 \% \mathrm{CI}$ & $\begin{array}{l}\text { p- } \\
\text { value }\end{array}$ & $\begin{array}{l}\text { P-value } \\
\text { (adjusted) }\end{array}$ \\
\hline $\begin{array}{l}\text { None -as per } \\
\text { intention to treat } \\
\text { analysis }\end{array}$ & 0 & 0 & 624 & 632 & 0.97 & $0.79-1.18$ & 0.7354 & 0.5296 \\
\hline $\begin{array}{l}\text { 1. Ineligible- } \\
\text { didn't meet all } \\
\text { inclusion criteria }\end{array}$ & 4 & 4 & 621 & 631 & 0.97 & $0.79-1.18$ & 0.7354 & 0.5296 \\
\hline $\begin{array}{l}\text { 2. Received no } \\
\text { trial medication }\end{array}$ & 7 & 11 & 616 & 629 & 0.98 & $0.80-1.20$ & 0.8510 & 0.6361 \\
\hline $\begin{array}{l}\text { 3. Received }<90 \\
\text { days of trial } \\
\text { medication due } \\
\text { to failure to } \\
\text { follow trial } \\
\text { procedures. }\end{array}$ & 4 & 15 & 614 & 627 & 0.98 & $0.80-1.20$ & 0.8232 & 0.5413 \\
\hline $\begin{array}{l}\text { 4. Received }<90 \\
\text { days of trial } \\
\text { medication as } \\
\text { chosen by } \\
\text { patient, relative, } \\
\text { or doctor, but } \\
\text { not for adverse } \\
\text { reactions. }\end{array}$ & 30 & 45 & 598 & 613 & 0.97 & $0.79-1.18$ & 0.7412 & 0.4604 \\
\hline $\begin{array}{l}\text { 5. Received }<90 \\
\text { days of trial } \\
\text { medication due } \\
\text { to suspected } \\
\text { adverse reaction } \\
\text { to the trial } \\
\text { medication. }\end{array}$ & 68 & 113 & 571 & 572 & 0.96 & $0.78-1.17$ & 0.6660 & 0.4068 \\
\hline $\begin{array}{l}\text { 6. Allocated to } \\
\text { placebo but } \\
\text { received an } \\
\text { SSRI for }>10 \\
\text { days within the } \\
\text { first } 90 \text { days }\end{array}$ & 13 & 126 & 571 & 559 & 0.89 & $0.72-1.10$ & 0.2678 & 0.1309 \\
\hline $\begin{array}{l}\text { 7. Allocated to } \\
\text { fluoxetine but } \\
\text { received an } \\
\text { SSRI for }>10 \\
\text { days within the } \\
\text { first } 90 \text { days. }\end{array}$ & 13 & 139 & 558 & 559 & 0.86 & $0.69-1.06$ & 0.1638 & 0.0783 \\
\hline $\begin{array}{l}\text { 8. Patients who } \\
\text { did not complete } \\
\text { at least } 150 \text { days } \\
\text { of treatment }\end{array}$ & 71 & 210 & 520 & 526 & 0.90 & $0.72-1.11$ & 0.3204 & 0.1600 \\
\hline
\end{tabular}


This per-protocol analysis sequentially excluded subgroups of patients who either did not meet our eligibility criteria or had incomplete adherence to the trial medication, and shows the effect of fluoxetine vs placebo on the primary outcome of the mRS at 6 months for each subgroup. 


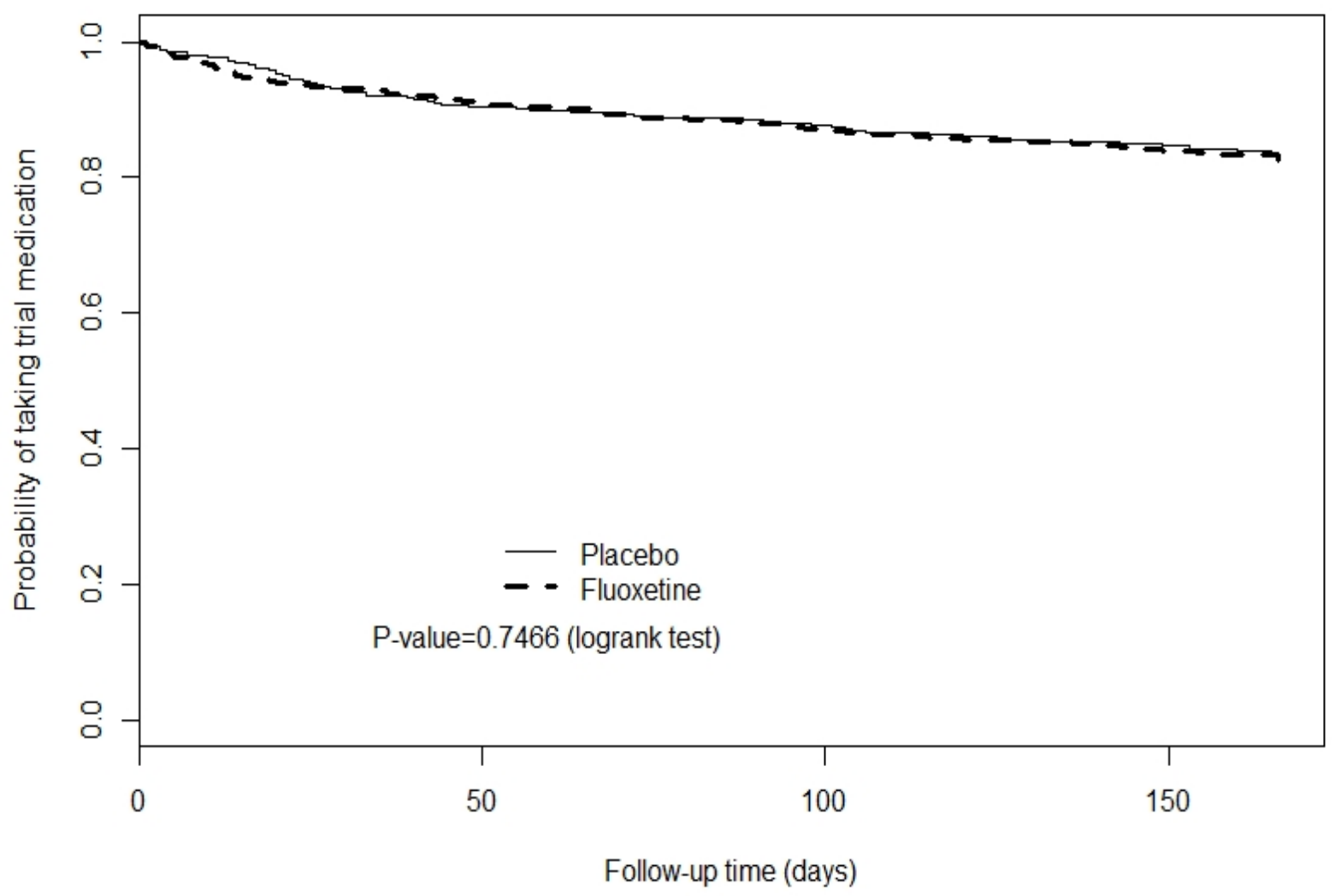

Supplementary figure 1.

Kaplan Meier curve of time to permanent discontinuation of trial medication 


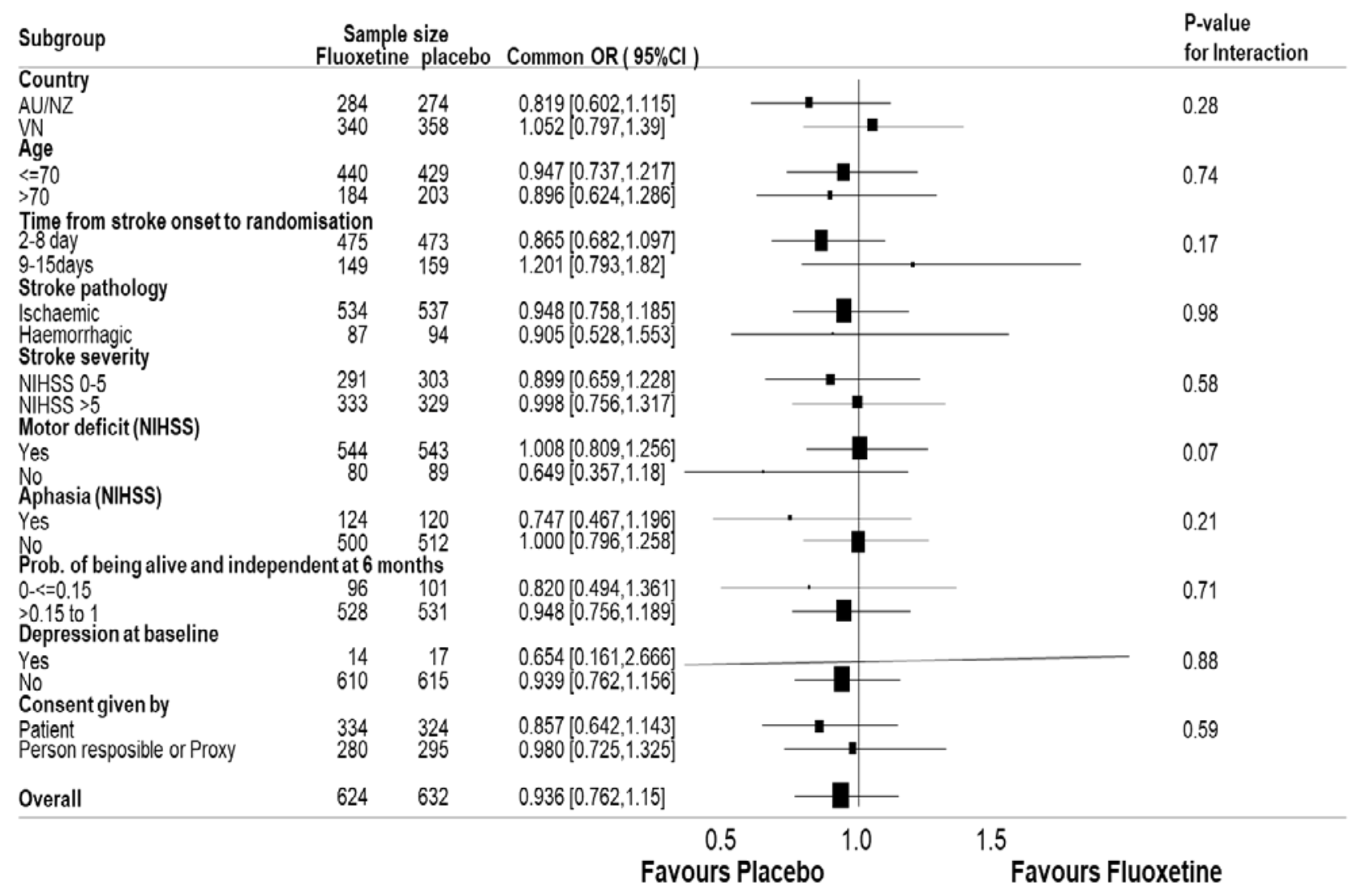

\section{Supplementary figure 2.}

\section{Primary outcome by pre-specified subgroups.}

The primary efficacy outcome was shift in the modified Rankin scale score distribution (range 0 [no symptoms] to 6 [death]) at 6 months (180 days). For subcategories, black squares represent point estimates (with the area of the square proportional to the number of events), and horizontal lines represent 95\% CIs. Scores on the NIHSS range from 0 to 42, with higher scores indicating more severe neurological deficits. Country: Country of randomization. AU/NZ: Australia, New Zealand. VN: Vietnam. NIHSS: National Institutes of Health Stroke Scale 


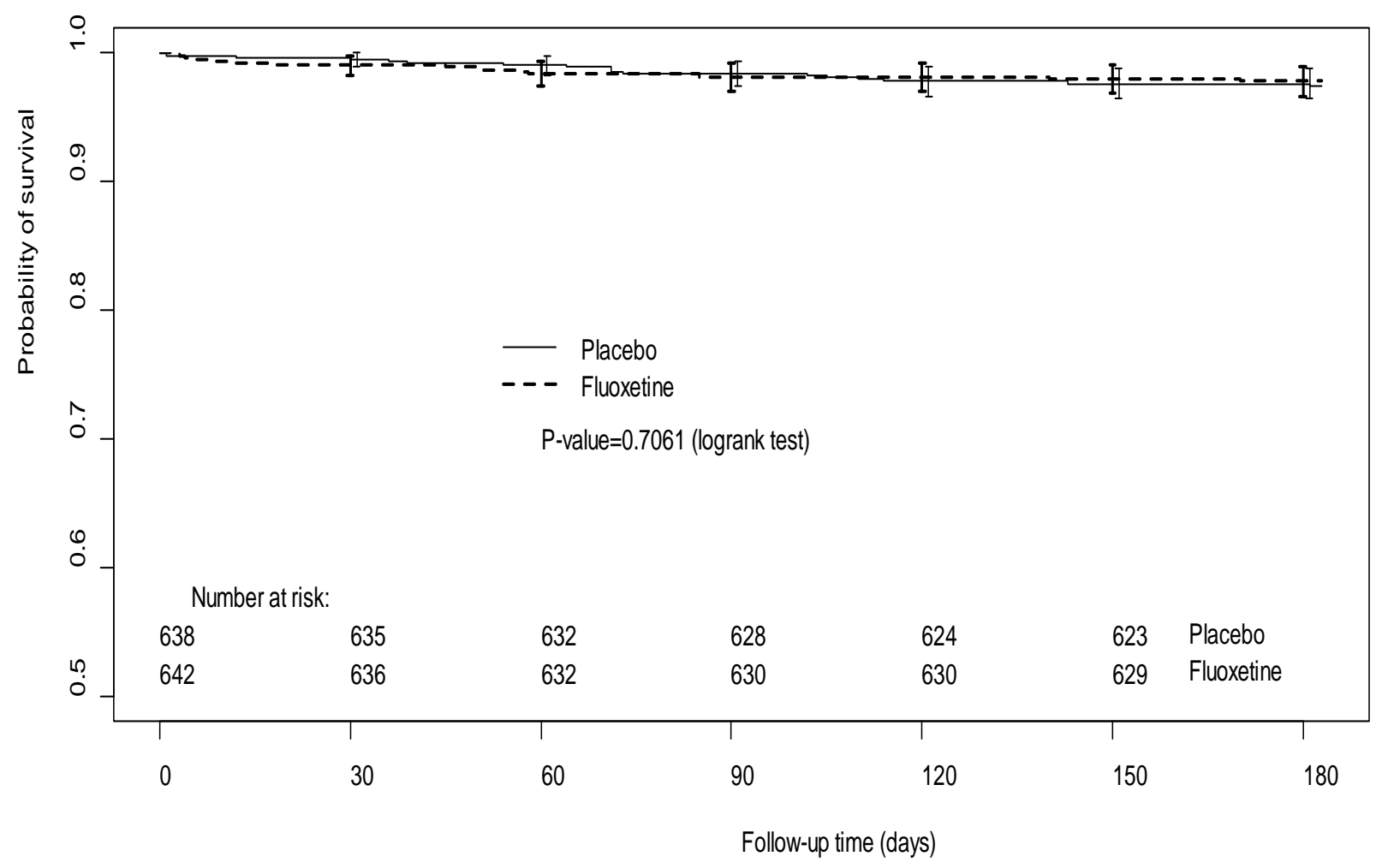

\section{Supplementary figure 3.}

\section{Kaplan Meier survival curve to 6 months follow-up.}

Patients who withdrew consent to be followed-up were censored at the time of withdrawal from the trial.

Estimates, their 95\% confidence intervals, and the number of patients at risk for each treatment group, are indicated for each time point after randomisation 


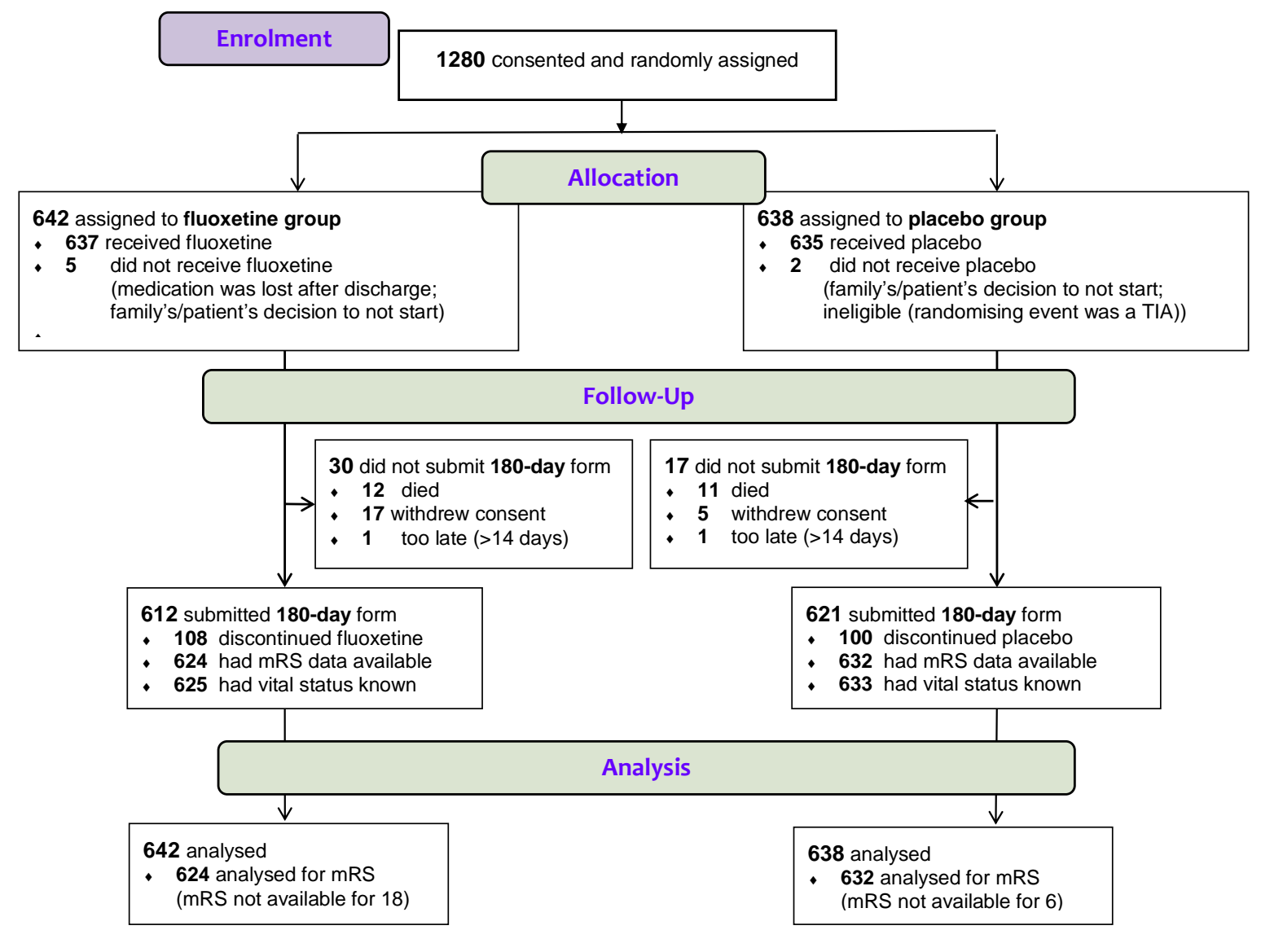

Figure 1: AFFINITY trial profile $\quad m R S=$ modified Rankin Scale 


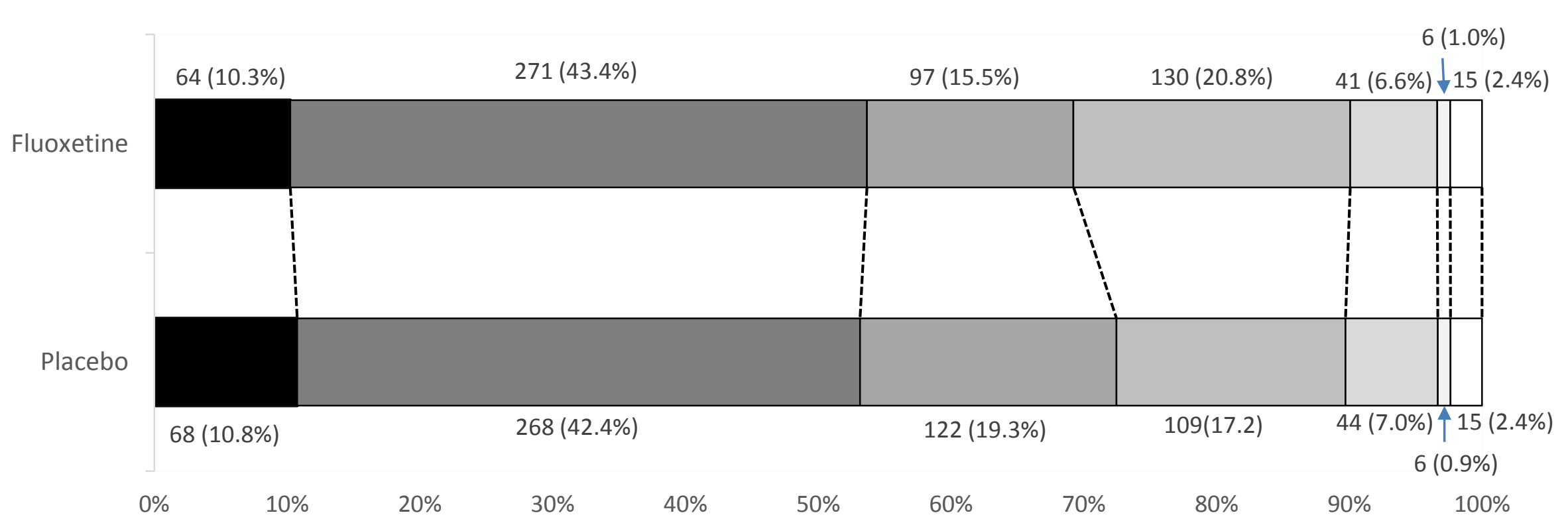

Figure 2. Primary outcome of the distribution of the modified Rankin Scale (mRS) scores at 6 months by treatment group 


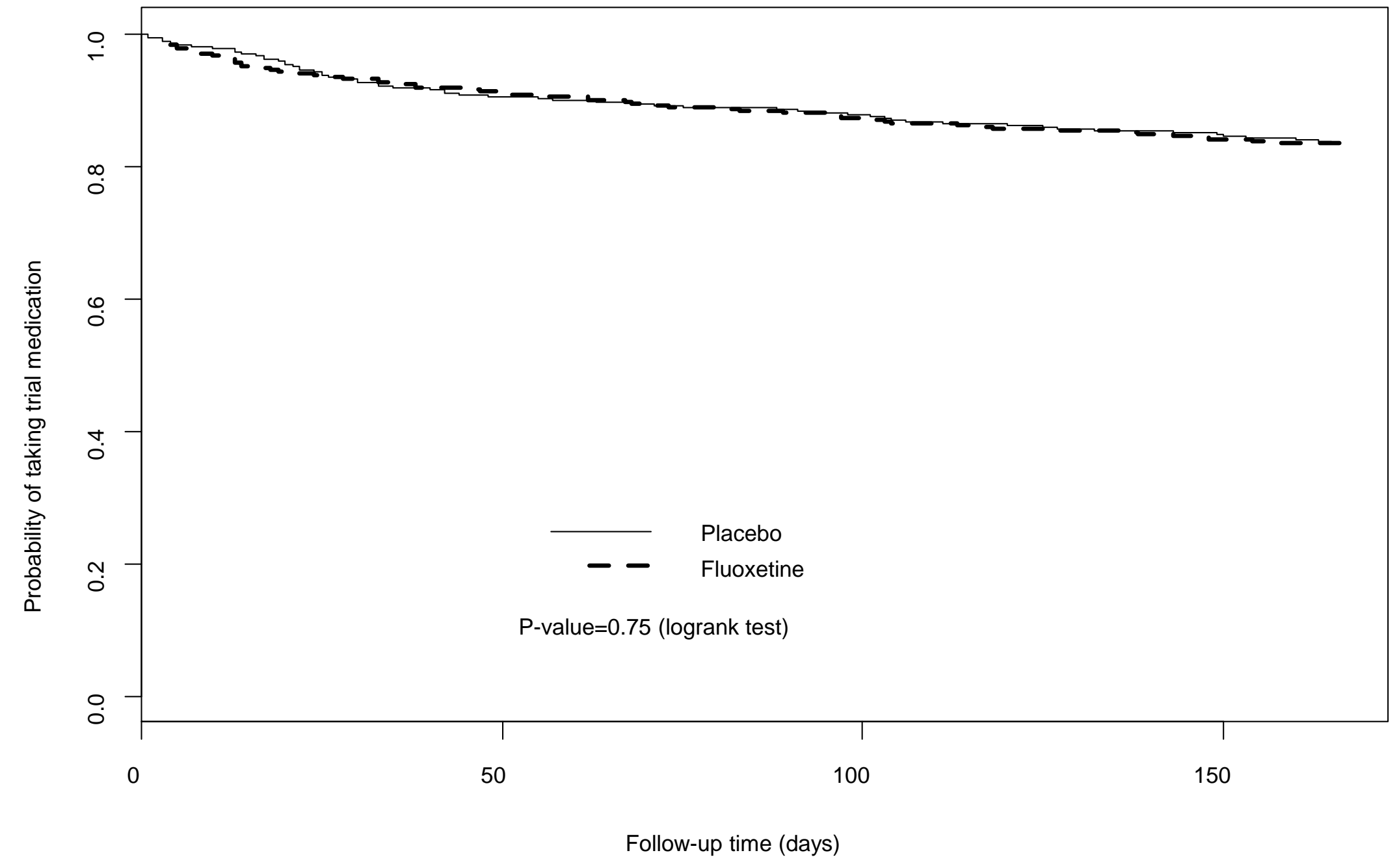

Supplementary figure 1. Kaplan Meier curve of time to permanent discontinuation of trial medication. 
$0.819[0.602,1.115]$
$1.052[0.797,1.39]$

$<=70$

$0.947[0.737,1.217]$

Time from stroke onset to randomisation

$0.896[0.624,1.286]$

9-15days

Stroke pathology

Ischaemic

Haemorrhagic

Stroke severity

NIHSS 0-5

Motor deficit (NIHSS)

$0.865[0.682,1.097]$

$1.201[0.793,1.82]$

$\begin{array}{rr}534 & 537 \\ 87 & 94\end{array}$

$0.948[0.758,1.185]$
$0.905[0.528,1.553]$

$291 \quad 303$

$0.899[0.659,1.228]$

Yes

No Aphasia (NIHSS)

Yes

$0.998[0.756,1.317]$

$1.008[0.809,1.256]$ $0.649[0.357,1.18]$

$124 \quad 120$

No

$\left.\begin{array}{l}0.747[0.467,1.196] \\ 1.000[0.796,1.258\end{array}\right]$

$0-<=0.15$

$>0.15$ to 1

Depression at baseline

Yes

Consent given by

500 months

$0.820[0.494,1.361]$

$0.948[0.756,1.189]$

$528 \quad 531$

$14 \quad 17$

$610 \quad 615$

Patient

$0.654[0.161,2.666$

$0.939[0.762,1.156$

$334 \quad 324$

$0.857[0.642,1.143$

$0.980[0.725,1.325]$

Overall

624

$0.936[0.762,1.15]$

]

0.5

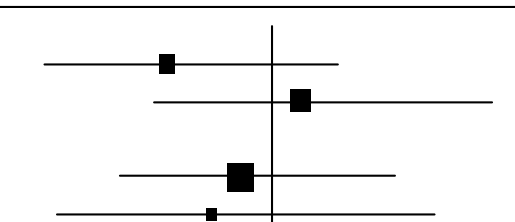

0.28

0.74

0.17

0.98

0.58

0.07

0.21

0.71

0.88

0.59

Favours Placebo

1.0

1.5

Favours Fluoxetine

Supplementary figure 2. Primary outcome by pre-specified subgroups. 


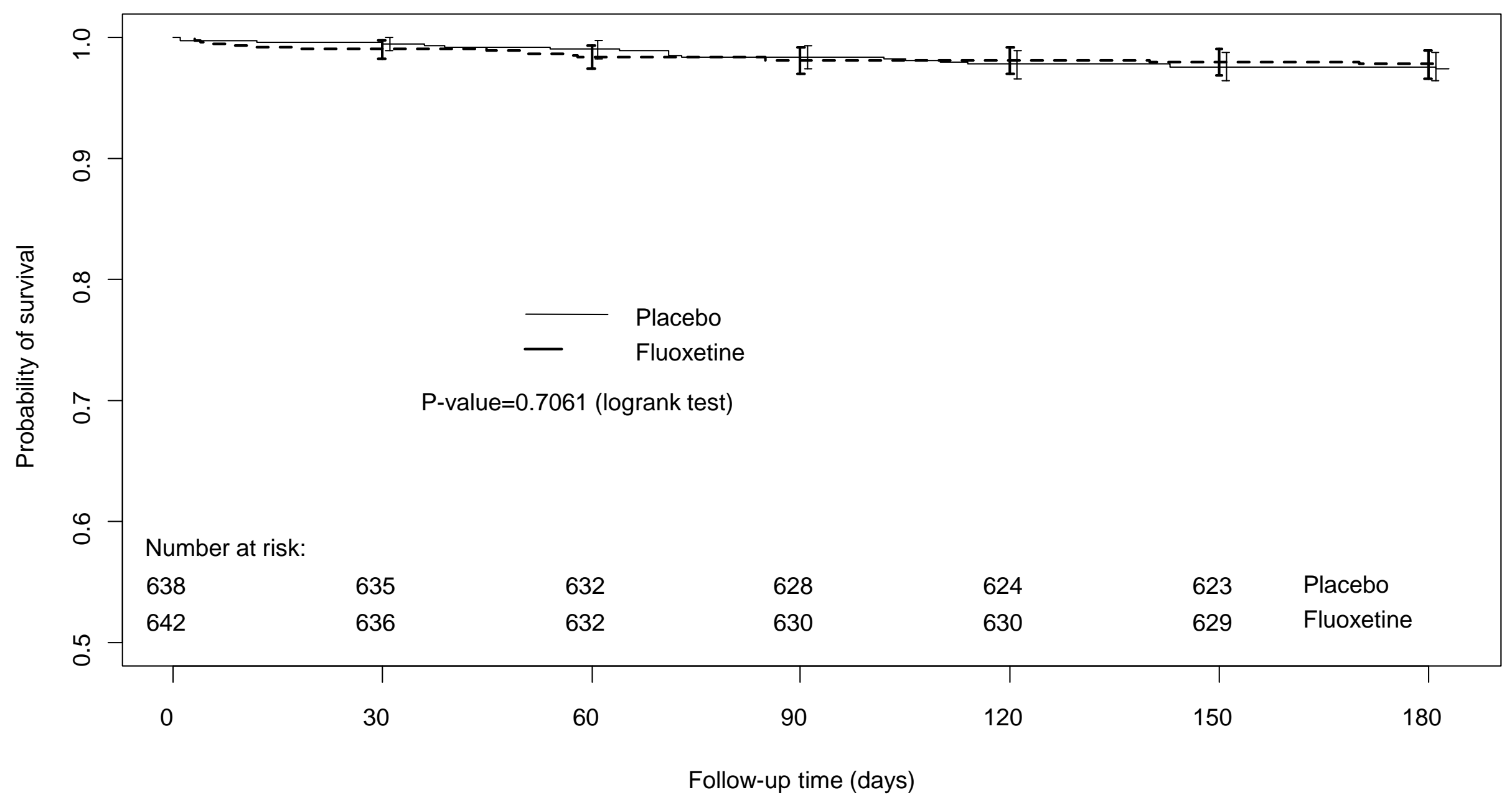

Supplementary figure 3. Kaplan Meier survival curve to 6 months follow-up.

Estimates, their $95 \%$ confidence intervals, and the number of patients at risk for each treatment group, are indicated for each time point after randomisation 
Manuscript reference number: THELANCETNEUROLOGY-D-20-00293

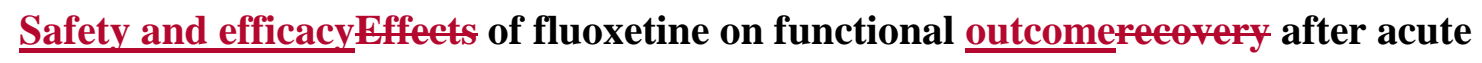
stroke (AFFINITY):

a randomised, double-blind, placebo-controlled trial.

\section{AFFINITY Trial Collaboration*}

*Members of the writing group are listed at the end of the Article;

all members of the AFFINITY trial Collaboration are listed in the appendix.

Summary: $\quad 34 \underline{5} 8$ words

Research in context: $39 \underline{0} 1$ words

Text: $\quad 4 \underline{597828}$ words

References: $\quad 30$

Tables: $\quad 3$

Figures: $\quad 2$

Supplementary appendix:

Tables: $\quad 5$

Figures: $\quad 3$

Correspondence to:

Professor Graeme J. Hankey

Professor of Neurology, Medical School, Faculty of Health and Medical Sciences,

The University of Western Australia

Room 222, Harry Perkins Institute of Medical Research

QQ Building, QEII Medical Centre,

6 Verdun Street, Nedlands, Perth, Western Australia,

Australia 6009

Email: graeme.hankey@uwa.edu.au 
Phone: +61861510828

\section{Summary}

\section{Background}

Trials of fluoxetine for recovery after stroke report conflicting results. The Assessment oF FluoxetINe In sTroke recoverY (AFFINITY) trial aimed to determine if daily fluoxetine for 6 months after stroke improves functional recoveryoutcome in Australasian and Vietnamese patients.

\section{Methods}

AFFINITY was a randomised, parallel-group, double-blind, placebo-controlled trial conducted in 43 hospital stroke units in Australia ( $\mathrm{n}=29)$, New Zealand (4), and Vietnam (10). Eligible patients were adults with a clinical diagnosis of stroke in the previous 2-15 days and a persisting neurological deficit. Patients were randomised via a web-based system using a minimisation algorithm to once daily, oral fluoxetine $20 \mathrm{mg}$ or matching placebo for 6 months. Patients, investigators and outcome assessors were masked to the treatment allocation. The primary outcome was functional recoveryoutcome, measured by the modified Rankin scale (mRS), at 6 months. The primary analysis was an ordinal logistic regression of the mRS at 6 months, adjusted for minimisation variables. Analyses were according to the patient's treatment allocation. The trial is registered with the ACTRN registry, number 12611000774921.

\section{Findings}

1280 patients were recruited in Australia $(n=532)$, New Zealand $(n=42)$ and Vietnam $(n=706)$ between 11 January 2013 and 30 June 2019; 642 were allocated fluoxetine and 638 placebo. Adherence to trial medication (mean 167 [SD 48] days) was similar between groups. At 6 months, mRS data were available in 624 (97.2\%) patients allocated fluoxetine and $632(99.1 \%)$ placebo. The distribution of mRS categories at 6 months was similar in the fluoxetine and placebo groups (adjusted common odds ratio 0.936, 95\% CI 0.762-1.150; p=0.53), and consistent among all pre-defined subgroups. Compared to placebo, patients allocated fluoxetine had more falls (20 [3.12\%] vs 7 [1.10\%]; $p=0.02)$, bone fractures $(19$ [2.96\%] vs 6 [0.94\%]; $\mathrm{p}=0.01)$ and epileptic seizures $(10[1.56 \%]$ vs $2[0.31 \%] ; \mathrm{p}=0.04)$ at 6 months.

\section{Interpretation}


Fluoxetine 20mg daily for 6 months after acute stroke did not improve functional recoveryoutcome and increased the risk of falls, bone fractures, and seizures. These results do not support the use,-or further trials, of fluoxetine to improve recoveryoutcome after stroke.

\section{Funding}

National Health and Medical Research Council of Australia (Project Grant 1059094). 


\section{RESEARCH IN CONTEXT}

\section{Evidence before this trial}

We undertook a Cochrane systematic review and searched Cochrane and clinical trial registers; MEDLINE, Embase, PubMed, and other biomedical databases; from their inception to 16 July 2018; for randomised controlled trials (RCTs) that recruited stroke patients who had survived up to one year, and randomised them to a selective serotonin reuptake inhibitor (SSRI), at any dose, for any period, and any indication; or to usual care or placebo. We identified 63 RCTs that compared any SSRI with control in 9168 stroke survivors. About half of the trials required patients to have depression. Potential improvements in disability with fluoxetine were only present in trials at high risk of bias. A meta-analysis of the three trials at low risk of bias $(\mathrm{n}=3356$ patients) found no effect of any SSRI compared to control on functional independence (risk ratio [RR] 1.00, 95\% CI 0.91 to $1.09 ; \mathrm{p}=0.99$ ) or disability score (standardised mean difference [SMD] $-0.01,95 \% \mathrm{CI}-0.09$ to $0.06 ; \mathrm{p}=0.75$ ). The evidence before this trial suggests that SSRIs do not improve functional recoveryoutcome after stroke, but doubt remains because this meta-analysis was dominated by one large trial, the FOCUS (Fluoxetine Or Control under Supervision) trial $(\mathrm{n}=3127)$, in UK patients.

\section{Added value of this trial}

The Assessment oF FluoxetINe In sTroke recoverY (AFFINITY) trial externally validates the the-FOCUS trial and Cochrane systematic review of RCTs of SSRIs for stroke recovery in an independent population of Australasian and Vietnamese stroke patients, reinforcing the conclusion that fluoxetine does not improve functional recoveryoutcome after stroke. The AFFINITY trial also adds further data regarding the potential hazards of treating acute stroke patients with fluoxetine $20 \mathrm{mg}$ daily for 6 months, including increased risks of falls, fractures, and seizures.

\section{Implications of all the available evidence}

For clinicians, SSRIs should not be prescribed routinely to improve functional recoveryoutcome after stroke because they are ineffective and increase serious adverse events. For researchers, a pooled analysis of individual patient data from completed RCTs of SSRIs for stroke recovery is needed to examine the effects of SSRIs in specific patient subgroups, such as those with hemiparesis, severe stroke, and cognitive impairment; and on specific outcomes, such as the mRS, motor domains of the Stroke Impact Scale, falls, fractures and 
seizures. Until these results are available, further trials of fluoxetine for stroke recovery are not recommended.

\section{Introduction}

Stroke is the second leading cause of disability-adjusted life years globally. ${ }^{1,2}$ Fluoxetine, a selective serotonin re-uptake inhibitor (SSRI), may improve neurological functional-recovery and reduce disability after acute-stroke. Fluoxetine exerts neuro-protective and neuroregenerative effects in pre-clinical models of acute brain ischaemia. ${ }^{3,4} \mathrm{~T}$ th the FLuoxetine for motor recovery After acute ischaeMic strokE (FLAME) trial found that, a double blind, randomised controlled trial (RCT) in in 118 patients with acute ischaemic stroke and-moderate to severe motor deficits, fluoxetine $20 \mathrm{mg}$ once daily significantly improved motor and functional-recovery afterever 3 months. ${ }^{5}$ A subsequent-Cochrane systematic review of 52 randomised controlled trials (RCTs) of SSRIs for stroke recovery in 4059 patients concluded that SSRIs may improve disability but, given methodological limitations and heterogeneity of the available-studies, more definitive,-larger trials were required. ${ }^{6}$

Hence, our international collaboration ve group designed and-undertook three elinicaltrials of fluoxetine for recovery after stroke in the United Kingdom (Fluoxetine Or Control Under Supervision [FOCUS]), Sweden (Efficacy of Fluoxetine-A Randomised Controlled Trial in Stroke [EFFECTS]) and Australia, New Zealand and Vietnam (Assessment oF FluoxetINe In sTroke recoverY [AFFINITY]). ${ }^{7,8}$ The FOCUS trial $(\mathrm{n}=3127)$ reported that fluoxetine $20 \mathrm{mg}$ given-daily for 6 months after stroke did not improve functional outcome as measured by the modified Rankin scale (mRS),s but reduced_the oceurrence of depression and increased the frequency of bone fractures. ${ }^{9}$ The latter results were consistent with the reported s of the effectiveness of fluoxetine as an antidepressant, ${ }^{10}$ and of an-increased risk of fractures in older people taking SSRIs. ${ }^{11,12}$ However, as only two thirds of FOCUS trial-patients adhered totøok the trial medication for at least 150 of the prescribed 180 days, a modest, but important, effect of fluoxetine trial medication-on functional outcome may have been missed. ${ }^{9}$ Moreover, as $96 \%$ of patients in FOCUS were white, the results may not be generalisable outside of the UK. ${ }^{9}$ Hence, the AFFINITY and EFFECTS trials continued to-recruitment patients in parallel-until 30 June, 2019.

Herein, we report the results of the AFFINITY trial, which aimed to evaluate whether a 6month course of fluoxetine is safe and effective, compared to placebo, for improving functional 
recoveryoutcome after recent stroke in an ethnically diverse population. The results of the EFFECTS trial results are reported in a parallel publication. ${ }^{13}$

\section{Methods}

Study design and participants

AFFINITY was a randomised, parallel group, double-blind, placebo-controlled clinical trial conducted in 43 hospital stroke units in Australia ( $n=29)$, New Zealand (4), and Vietnam (10). The trial protocol (appendix) was approved by the Royal Perth Hospital Ethics Committee on 24 February, 2012 (approval number EC2011/131), and subsequent amendments to the protocol to facilitate trial recruitment-were also approved. All participating sites received approval from their ethics committee and institutional review board. The trial protocol $^{7}$ and statistical analysis plan ${ }^{8}$ were published before recruitment stopped-was completed.

Eligible patients were adults (aged $\geq 18$ years) with a clinical diagnosis of acute stroke within the previous 2-15 days, brain imaging consistent with ischaemic or haemorrhagic stroke (including a normal CT brain sean), and a persisting neurological deficit that produced a modified Rankin scale (mRS) score $\geq 1$. Patients were excluded if there was any definite indication for fluoxetine-(e.g. depression), or contraindication to fluoxetine (e.g. history of epilepsy, bipolar disorder, drug overdose, allergy to-fluoxetine_allergy, or any-recent medication that could interact with fluoxetine; or biochemical evidence of hepatic impairment [serum alanine aminotransferase $(\mathrm{ALT})>120 \mathrm{U} / \mathrm{l}$ ], renal impairment [creatinine $>180 \mu \mathrm{mol} / \mathrm{l}$ or estimated glomerular filtration rate $(\mathrm{eGFR})<30 \mathrm{ml} / \mathrm{min} / 1.73 \mathrm{~m}^{2}$ ], or hyponatremia [sodium $<125 \mathrm{mmol} / \mathrm{L}]$ ); if patients were unlikely to be available for follow-up during the subsequent 12 months; if patients had another life-threatening illness that would make 12-month survival unlikely [e.g. terminal malignancy]; if patients were-women wereand pregnant, breast-feeding or of child-bearing age and not without the usinge of contraception; or if patients were enrolled in another clinical trial of an investigational medicinal product (IMP) or device.

Written informed consent was obtained from each patient or, if the patients were unable to provide consent, from their legally approved surrogate.

\section{Randomisation and masking}


The patient's clinician entered the patient's baseline data (table 1) into a secure, passwordprotected, centralised, web-based randomisation system which checked the data for completeness and consistency and then-generated a unique study identification number and treatment pack number which-correspondinged to fluoxetine or placebo in a 1:1 ratio. A minimisation algorithm ${ }^{14}$ was used to achieve balance between the treatment groups in four predictors of the primary outcome (mRS): time after stroke onset (2-8 vs 9-15 days), presence of a motor deficit (National Institutes of Health Stroke Scale [NIHSS] questions 5 and 6), presence of aphasia (NIHSS question 9), and probability of survival free of dependency (mRS $0-2)$ at 6 months $(0.00$ to $\leq 0 \cdot 15$ vs $>0 \cdot 15-1.00)$ as -calculated using a previously-validated prognostic model comprising six baseline prognestic-variables (age, living alone before the stroke, independent in activities of daily living before the stroke, and able to talk, lift both arms off the bed, and walk unassisted at the time of -randomisation). ${ }^{15}$

All patients, carers, investigators, and outcome assessors were masked to the allocated treatment by use of placebo capsules that were visually identical to the fluoxetine capsules even when broken open.

\section{Procedures}

Fluoxetine $20 \mathrm{mg}$ capsules or matching placebo capsules were administered to patients-orally, once daily, for 6 months. If the patients wereas unable to swallow, the capsules werecould be broken open and the-contents administered via an enteral feeding tube.

Siegfried Malta Ltd, Hal Far, Malta, manufactured the capsules containing fluoxetine 20mg according to Good Manufacturing Practice (certificate MT/008HM/2017). Arena Pharmaceuticals $\mathrm{GmbH}$, Zofingen, Switzerland, packed the capsules for Amneal Pharmaceuticals Pty Ltd, South Yarra, Australia, which was the Therapeutic Goods Administration (TGA) licence holder (sponsor) for the finished product in Australia. Pharmaceutical packaging professionals (PPP) Pty Ltd, Port Melbourne, Australia purchased the fluoxetine capsules and manufactured the matching placebo capsules. PPP packaged the trial medication in patient kits, labelled the bottles with trial-specific treatment codes (fluoxetine or placebo), and packaged, stored and distributed the medication. The patient kits in Australian and New Zealand comprised two bottles, each containing 110 capsules, which 
were dispensed at randomisation and day 90. An extra 20 capsules were a reserve in the event of any delay in attending the day 90 follow-up, or any loss or spillage of capsules. For patients in Vietnam, the kits comprised 6 bottles of trial medication, each containing 35 capsules. One bottle was dispensed at randomisation, two bottles at day 28 , and three bottles at day 90 . The TGA of the Australian government's Department of Health approved the export of trial medication to New Zealand and Vietnam (approval Ref No: EX17/336513).

All patients received organised, interdisciplinary care and rehabilitation in stroke units.

Patients recruited in Australia and New Zealand were assessed by site investigators at 28 days (1 month) and 90 days ( 3 months) post-randomisation in the hospital ward or outpatient clinic or via telephone or email; or, failing that, by a study nurse at the patient's residence. Followup at 180 days ( 6 months) was by postal questionnaire or telephone by trained staff in the trial coordinating centre in Perth, Australia. Patients recruited in Vietnam were assessed by the site investigator at 28, 90 and 180 days post-randomisation in the hospital ward or outpatient clinic, or via telephone or email; or, failing that, at the patient's residence. If the patient was unable to complete the assessments, assistance was sought from their proxy (next of kin, close family member or carer). Each assessment recorded the primary outcome (mRS), secondary outcomes (table 2), safety and adverse events (table 3), all current medications, and adherence to trial medication. Serum sodium, eGFR, and liver function were measured at the first (28 day) follow-up visit if clinically appropriate. Adherence to trial medication was assessed by asking: 'On average, since the last follow-up, how many times per week was the trial medication taken? '0, 1-2, 3-4, 5-6 or 7 times per week'; and by pill counts and collection of returned trial bottles. Bottle and pill counts were conducted by hospital trial pharmacists, and entered on a drug accountability form. Any interruption to trial medication was recorded as temporary or permanent, together with the dates and reasons for stopping and re-starting.

If patients developed new depression requiring treatment during the trial treatment period, the protocol recommended continuation of trial medication and consideration of nonpharmacological (e.g. psychological) interventions. If antidepressant medication was eonsiderednecessary, referral to a psychiatrist was recommended for consideration of potential interactions of any new medication with fluoxetine and risks of serotonin toxicity. 
There were 57 protocol violations in 56 patients (4.4\%): $20(1.6 \%)$ patients were prescribed open-label fluoxetine; $8(0.6 \%)$ were prescribed another SSRI; $16(1.3 \%)$ lost their trial medication or did not take it the trial medication-as prescribed; $4(0.3 \%)$ took medications that could interact with fluoxetine (e.g. antipsychotic, tramadol); 7 (0.5\%) patients were $\geq$ more than 14 days late for a-scheduled follow-up; one patient enrolled in another trial was deemed ineligible, and four patients had a final diagnosis other than stroke (table 1). Emergency unblinding was not necessary for any patient.

\section{Outcomes}

The primary outcome was functional status, as measured by the mRS, at 6 months after randomization. ${ }^{16}$ The mRS is an ordinal scale which assigns patients to seven ordered, but not equally spaced, levels of functional ability, ranging from 0 (symptom free) to 6 (dead).

Secondary outcomes at 6 months were survival, depression (PHQ-9 score $\geq 15^{17}$ ), cognition (Telephone Interview for Cognitive Status [TICSm] ${ }^{18}$ ), communication, motor function, overall health status (Stroke Impact Scale [SIS] version 3.0 ${ }^{19}$ ), fatigue (vitality subscale of the SF-36 ${ }^{20,21}$ ) and health-related quality of life (HRQoL) using the EQ-5D-5L. ${ }^{22}$ A new diagnosis of depression requiring treatment with antidepressants was assessed at 1, 3 and 6 months by asking patients if they had been diagnosed with depression since each of their previous assessments, and verifying the diagnosis and treatment plan-with their clinician.

Serious adverse events at any time-during follow-up included recurrent stroke (ischaemic or haemorrhagic), acute coronary syndromes, upper gastrointestinal bleeding requiring blood transfusion and/or endoscopy, other major bleeding (subdural, extradural, ocular, lower gastrointestinal) requiring blood transfusion or procedural intervention, falls with injury, new bone fractures, epileptic seizures, symptomatic hypoglycaemia (blood glucose $<3 \mathrm{mmol} / \mathrm{l}$ ), symptomatic hyperglycaemia (blood glucose $>22 \mathrm{mmol} / \mathrm{l}$ ), new hyponatraemia (blood sodium $<125 \mathrm{mmol} / \mathrm{l})$, attempted suicide or self-harm, and death.

\section{Statistical analysis}


A detailed statistical analysis plan was formulated and published before recruitment was completed and without awareness of any unblinded data. ${ }^{8}$

We estimated from other concurrent studies of patients with acute stroke that $42.2 \%$ of patients assigned placebo would be functionally independent (mRS 0-2) at 6 months after randomisation. ${ }^{23,24}$ We calculated the odds ratio (OR) of functional independence (mRS 0-2) with fluoxetine vs placebo in the FLAME study ${ }^{5}$ to be 3.57 (95\%CI: 1.2 to 10.6). We considered a conservative estimate of the effect of fluoxetine may be toward the lower $95 \% \mathrm{CI}$ of the OR estimate reported in FLAME (e.g. OR 1.34). If fluoxetine increased the proportion of patients who were functionally independent at 6 months by an OR of 1.34, from $42.2 \%$ (placebo) to $49.4 \%$ (fluoxetine), this would be clinically important and consistent with our Cochrane review. ${ }_{-}^{6}$ - that is, a $7.2 \%$ absolute increase in functional independence in patients allocated fluoxetine compared with placebo. Assuming a common OR of 1.34 for each cutpoint across the mRS (e.g. 0 vs 1-6, 0-1 vs 2-6, etc) in the proportional odds logistic model, we estimated that the trial would require 1600 patients to have $90 \%$ power, if up to $10 \%$ of patients dropped out before final follow-up (i.e. 1440 patients with primary outcome data). ${ }^{8,25}$

All analyses, including primary and secondary outcomes and adverse events, were by intentionto-treat, according to the treatment allocation. A secondary safety analysis was undertaken according to the treatment patients received rather than what they were randomly allocated.

The primary analysis was an ordinal analysis of the mRS scores at 6 months in each treatment group using ordinal logistic regression and after adjusting for the baseline factors included in the minimisation algorithm. ${ }^{8}$ The ordinal analysis of mRS was undertaken by treatment allocation, under the assumption of proportional odds in the model. The result was expressed as a common OR (less than 1.0 favoured placebo) and its $95 \%$ confidence interval (CI). We also performed 6 binary unadjusted logistic regressions, each corresponding to the 6 possible dichotomisations of scores on the mRS.

Secondary analyses compared the following outcomes at 6 months follow up-in each treatment group: survival, depression (changes in PHQ-9 scores and proportion with PHQ-9 $\geq 15$ ), ${ }^{17}$ cognition (TICSm scores), ${ }^{18}$ communication (SIS), ${ }^{19}$ motor function (SIS), ${ }^{19}$ overall health status (SIS), ${ }^{19}$ HRQoL (EQ-5D-5L), ${ }^{22}$ new diagnosis of depression requiring treatment with 
antidepressants, fatigue (vitality domain of the SF-36) ${ }^{20,212}$, trial medication adherence and cessation, and serious adverse events. The frequencies of categorical outcome events_; including adverse events, in each treatment group were compared using Fisher's exact test. For continuous outcomes, the mean or median in each group, depending on the distribution, were calculated with measures of dispersion (standard deviation [SD] or inter-quartile range [IQR]). The probability that outcome-measures in the fluoxetine reatment-group were significantly different from those observed in-the placebo group were calculated and expressed-as p-values.

Pre-specified subgroup analyses of the effect of fluoxetine vs placebo on the primary outcome were undertaken for key baseline variables, including country of randomisation (Australia/New Zealand vs Vietnam), age ( $\leq 70$ vs $>70$ years), time from stroke onset to randomisation (2-8 vs 9-15 days), stroke pathology (ischaemic vs haemorrhagic), stroke severity (NIHSS scores $\leq$ median $[0-5]$ vs $>$ median [ $>5]$ ), motor deficit (present vs absent), aphasia (present vs absent), probability of survival free of dependency $(0.00$ to $\leq 0.15$ vs $>0.15$ to 1.00$)$, self-reported depression at baseline, and source of informed consent. ${ }^{8}$

We also undertook pre-specified per-protocol analyses, which sequentially excluded subgroups of patients who did not meet our eligibility criteria or had incomplete adherence to the trial medication.

Post-hoc sensitivity analyses of the primary outcome were undertaken to evaluate the possible effect of including these patients who were lost to follow-up. We tested the robustness of the results by assuming two extreme imputation scenarios: one favouring fluoxetine where all patients with a-missing mRS data were imputed a score of 0 in the fluoxetine arm and a score of 6 in the placebo arm, and another scenario favouring placebo where the imputation was reversed.

Statistical analyses were undertaken with SAS, version 9.4

An independent data monitoring committee (DMC) oversaw the study. The unmasked trial statistician (Q Yi) prepared analyses of the accumulating data, which the DMC reviewed in strict confidence at least_-annuallyønce a year. 
The trial is registered with the Australian New Zealand Clinical Trials Registry, number ACTRN12611000774921.

\section{Role of the funding source}

The funder of the study had no role in study design, data collection, data analysis, data interpretation, or writing of the report. The corresponding author had full access to all the data in the study and had final responsibility for the decision to submit for publication.

\section{Results}

A total of 1280 patients consented and were randomised at 43 sites in Australia, New Zealand and Vietnam between 11 January, 2013 and 30 June, 2019. Recruitment was terminated before the target sample size-of 1600 patients was reached becauseas grant_funding expired on 31 December 2019.

642 patients were randomly allocated to fluoxetine and 638 to placebo. One patient did not meet our eligibility criteria after randomisation because of later discovery of participation in another elinical-trial of an_-IMPinvestigational medicinal product. In four patients, the initial diagnosis was later revised to be non-stroke_after review of investigations at the one month follow up-(table 1).

Baseline characteristics in the two treatment groups were balanced (table 1)

By 6 months, $22(1.7 \%)$ patients had withdrawn consent for follow-up and a further $2(0.2 \%)$ patients-were lost to follow-up (fig 1). There was no difference in the methods of follow-up between treatment groups (appendix table 1, p8). Trial medication was started in 1273 patients, and it was-temporarily and permanently stopped in 158 and 208 patients respectively_, before the 6 month follow up-(appendix, table 2, p9). There was no significant difference between treatment-groups in temporary and permanent discontinuation of trial medication (appendix table 2, p9) or time to permanent discontinuation ( $\mathrm{p}=0.75$; appendix figure $1, \mathrm{p} 14)$. There were also no significant-differences between groups in compliance with trial medication at one $(p=0.57)$, three $(p=0.94)$ or $\operatorname{six}(p=0.92)$ months $($ appendix table $3, p 10)$. The mean duration of trial treatment during the 6 months follow up-was 167 days (SD 48.1) days. 
The primary outcome, the mRS at 6 months, was assessed and analysed in 624 (97.2\%) patients allocated fluoxetine and $632(99.1 \%)$ placebo. An ordinal comparison of the distribution of patients across the $\mathrm{mRS}$ categories at 6 months, adjusted for variables included in the minimisation algorithm, was similar in both the two-groups (common OR 0.936, 95\% CI 0.7621.150; $\mathrm{p}=0.53$; figure 2). A common $\mathrm{OR}<1.0$ favours placebo. The unadjusted analysis produced similar results (common OR 0.966, 95\%CI 0.790-1.181; $\mathrm{p}=0.74$; appendix table 4, p11). The assumption of proportional odds in the model of mRS by treatment was upheld in the score test for proportional odds assumption ( $\mathrm{p}=0.44$ unadjusted). Comparison of Ddichotomised mRS scores ( $\left(\begin{array}{llll}2 & \text { vs } & 3 & 6\end{array}\right)$-were also showed-not significantly differentee between treatment groups for mRS 0-2 vs 3-6 (unadjusted OR 0.855, 95\%CI 0.670-1.091; $\mathrm{p}=0.21$; post-hoc adjusted $\mathrm{OR}=0.823,0.628-1.077 ; \mathrm{p}=0.16$; appendix table 4 , $\mathrm{p} 11$ ), or . There was also no difference between groups in other dichotomies of the mRS (appendix table 4, p11).

Analysis of the primary outcome showed no significant interactions or modification of the effect of fluoxetine across several pre-specified subgroups (appendix figure 2, p15).

Secondary efficacy outcome-measures at 6 months are shown in table 2. Patients allocated fluoxetine had better mood and emotional control, as measured by-higher scores in the SIS domain of mood and Kemotional control scompared to placebo $(\mathrm{p}=0.003)$, but there were no significant differences between treatment groups in the other 10 domains of the SIS (including measures of motor function [strength, hand ability, mobility] and daily activities), other assessment scales, or death. There was a reduction in new diagnoses of post-stroke depression which was not statistically significant (33 [5.14\%] fluoxetine vs 46 [7.21\%] placebo; absolute risk difference $2.07 \%$; $95 \%$ CI $-0.57 \%$ to $4.41 \%]$ ).

Adverse events at 6 months are shown in table 3. Compared to patients allocated placebo, those allocated fluoxetine had more falls causing injury (20 [3.12\%] vs 7 [1.10\%]; difference $2.02 \%$ [95CI: 0.45-3.59]; $\mathrm{p}=0.02)$, bone fractures (19 [2.96\%] vs 6 [0.94\%]; difference $2.02 \%$ [0.51$3.53] ; \mathrm{p}=0 \cdot 01)$ and epileptic seizures (10 [1.56\%] vs 2 [0.31\%]; difference $1.24 \%$ [0.19-2.30]; $\mathrm{p}=0.04)$. There were no significant differences between treatment groups in other events, at, or during, the 6 months follow up,including survival $(\mathrm{p}=0.71$, appendix, figure $3, \mathrm{p} 16)$. Trial medication was stopped by 68 patients ( 27 allocated fluoxetine, 41 placebo) due to a suspected 
adverse reaction to the medication. No patients required a reduction in dose of trial medication (e.g. alternate daily) and there were no treatment-related deaths.

The primary results were not altered by sensitivity analyses confined to patients who adhered to the trial protocol and allocated treatment (appendix table 5, p12).

A post-hoc analysis, which consisted of imputing missing mRS data under two extreme scenarios, also produced eonsistent, non-significant results for the most extreme scenarios in favour of fluoxetine (unadjusted OR 1.082, 95\%CI 0.887-1.320; $\mathrm{p}=0.44$; adjusted OR 1.054, 0.861-1.291; $\mathrm{p}=0.61$ ), and for the most extreme scenario-in favour of placebo (unadjusted $\mathrm{OR}$ 0.860, 0.705-1.050; $\mathrm{p}=0.14$; adjusted OR 0.833, 0.680-1.020; $\mathrm{p}=0.09$ ).

\section{Discussion}

The main finding of the AFFINITY trial was that adding fluoxetine $20 \mathrm{mg}$ daily for 6 months after acute stroke to interdisciplinary stroke unit care did not improve functional outcome at 6 months in an ethnically diverse population. Other major findings were that fluoxetine improved moed and emotional contrel butincreased falls, fractures, and epileptic seizures at 6 months.

Key strengths of the trial are that it was undertaken in stroke units throughout Australia, New Zealand and Vietnam where the AFFINITY trial medication was added to best-practice, comprehensive interdisciplinary stroke care and rehabilitation. Several potential sources of systematic error (bias) in the assessment of fluoxetine vs placebo were minimised. Systematic pre-treatment differences in comparator groups (selection bias) were minimised by concealed, central, web-based randomisation. Adherence to trial medication was high and similar between treatment groups (performance bias). Systematic differences between groups in other care provided (performance bias) and reporting and assessment of outcome events (observer detection bias) were minimised by masking of patients, investigators and adjudicators to the allocated treatment. Follow up for the primary outcome was high and there was no difference between groups in withdrawals from treatment (attrition bias). Random error was reduced to some extent by almost complete follow-up of a large number of patients $(n=1256,98 \%)$, which was a higher proportion than planned in our sample size calculations (90\% of 1600; $n=1440$ ). The inclusion of an international mix of ethnic groups managed in different health care systems, 
and a comprehensive array of secondary outcome measures, including cognition, mood and motor scales, support the external validity (generalisability) of the trial results.

Potential limitations of the trial include our failure to recruit the target sample size of 1600 patients due to funding constraints (1280 patients recruited; 1256 with primary outcome data vs 1440 planned to have primary outcome data). We also failed to recruit a larger number of patients with severe, disabling stroke. Hence, the proportion of patients assigned placebo who recovered functional independence (mRS 0-2) at 6 months was higher $(n=458,72 \%)$ than estimated in our sample size calculations (42\%). The dose of fluoxetine was $20 \mathrm{mg}$ once daily because this was the dose reported to be effective in the FLAME trial ${ }^{5}$ and used in other fluoxetine trials for stroke recovery, ${ }^{6}$ and is less likely to cause adverse effects than higher doses. However, we did not test higher doses of fluoxetine. Our measures of adherence to trial medication by self-report and capsule-counting were prone to error (e.g. the absence of tablets in the bottles returned to investigators may not necessarily mean adherence to taking the tablets) and therefore, our estimates of adherence and compliance may be inflated. However, there was no difference between groups in reported adherence to, and discontinuation of, trial medication. The nature and degree of adjunctive rehabilitation was not documented because that would have added complexity and potential measurement error to this pragmatic trial. However, all patients were admitted to stroke units where organised interdisciplinary assessment, and rehabilitation as required, was provided as standard practice. The nature and intensity of all rehabilitation interventions were likely to be balanced between the treatment groups, in the same way that all baseline variables were balanced between groups, due to the randomisation process and double-blind trial treatment allocation. There was a slight difference in ascertainment of mRS status at 6 months between groups (fluoxetine $n=624,97.2 \%$ vs placebo $n=632,99.0 \%$ ) but sensitivity analyses using imputations led to consistent conclusions. Our primary measure of efficacy was a broad measure of functional outcome which may not be sensitive to changes in measures of specific neurological functions, However, we also measured 11 domains of the SIS, including measures of motor function (strength, hand ability, mobility), physical function, and daily activities, and found no effect of fluoxetine on any of these measures. The mRS may be less sensitive to change in patients with less severe stroke but there was no evidence of an effect of fluoxetine on the $\mathrm{mRS}$ in patients with more severe 
stroke (NIHSS > 5; appendix figure 2, p15), and no effect of fluoxetine on any secondary outcome except mood and emotional control.

The AFFINITY trial was smaller than the FOCUS trial ${ }^{9}$ but both trials recruited patients of similar sex (women 38\%), stroke severity (median NIHSS=6) and at a similar time (one week, mean) after stroke onset. The AFFINITY trial population was a unique mix of Vietnamese $(\mathrm{n}=727,57 \%)$ and Australasians $(\mathrm{n}=553,43 \%)$, whereas the FOCUS population was predominantly Caucasian $(n=2988 ; 96 \%)$. Patients in AFFINITY were also younger (mean age 64 years AFFINITY, vs 71 years in FOCUS), and more likely to be married ( $\mathrm{n}=926,72 \%$ vs $\mathrm{n}=1725,55 \%)$, living with someone else $(\mathrm{n}=1120,87 \%$ vs $\mathrm{n}=2091,67 \%)$, employed $(\mathrm{n}=531$, $41 \%$ vs $n=691,22 \%$ ), and independent before their stroke ( $n=1264,99 \%$ vs $n=2866,92 \%$ ) compared to FOCUS. Adherence to trial medication was higher in AFFINITY than FOCUS; $34(5.4 \%)$ patients assigned fluoxetine and $30(4.8 \%)$ placebo stopped trial medication within the first 90 days, whereas in FOCUS, 143 (9\%) patients assigned fluoxetine and 122 (8\%) placebo stopped trial medication within the first 90 days. Despite these differences, the results of the AFFINITY trial almost replicate those of the largerFOCUS trial, ${ }^{9}$ supporting the internal and external validity of both trials. Furthermore, the EFFECTS trial of fluoxetine vs placebo in 1500 stroke patients in Sweden also reports very-similar results to FOCUS and AFFINITY. ${ }^{13}$ Moreover, the results of the FOCUS, AFFINITY and EFFECTS trials are all consistent with the totality of evidence from all RCTs of SSRIs for stroke recovery, ${ }^{26}$ and all RCTs specifically testing fluoxetine. ${ }^{27}$ Collectively, these trials provided compelling evidence that fluoxetine does not improve functional recoveryoutcome after stroke.

The outstanding inconsistency among all the RCT evidence is the FLAME trial, which did

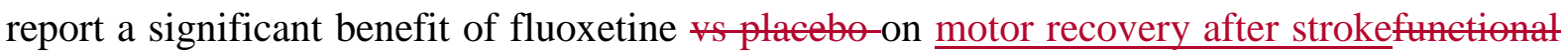
recovery measured by the mRS. ${ }^{5}$ The FLAME trial differed from AFFINITY, FOCUS and EFFECTS in that in that it was a phase II trial of fluoxetine $20 \mathrm{mg}$ daily vs placebo in a highly select population of $11 \underline{8} z$ patients with recent (5-10 days) ischaemic stroke and a moderate to severe hemiparesis or hemiplegia, defined by a Fugl-Meyer motor scale (FMMS) score $\leq 55 .^{5}$ The FMMS motor score ranges from 0 (hemiplegia) to a maximum of 100 points (normal motor performance), with divided into-66 points for the upper extremity and 34 points for the lower extremity. Patients enrolled in FLAME had more severe strokes (mean baseline NIHSS 13 vs median NIHSS 6 in AFFINITY).At baseline, there was some imbalance between the treatment 
groups; the mean total FMMS score was higher (better motor performance) in the fluoxetine group (17.1; SD 11.7) than placebo group (13.4; SD 8.8), and the mean total NIHSS was marginally lower (less severe stroke) in the fluoxetine group (12.8; SD 3.9) than placebo group (13.1; SD 4.3$).{ }^{5}$ Somatosensory and other neurological deficits that may influence recovery were not reported. The primary outcome-measure, the mean change in FMMS scores between randomisation and day 90 , was greater with fluoxetine than placebo (34.0 points fluoxetine vs 24.3 points placebo; difference 9.8 points, $95 \%$ CI: 3.4 to $16.1, \mathrm{p}=0.003)$. The proportion of functionally independent patients (mRS scores 0-2) at day 90 was also higher with fluoxetine than placebo $(\mathrm{n}=15,26 \%$ vs $\mathrm{n}=5,9 \% ; \mathrm{p}=0.02)$, but there were no differences between fluoxetine and placebo for other mRS categories. The FLAME trial result may be a falsepositive due to random error (chance), as only 57 patients were treated with fluoxetine and followed-up to 90 days, ${ }^{5}$ and there is large variation in spontaneous motor recovery after acute stroke. ${ }^{28}$ Alternatively, the FLAME trial result may a true-positive, and fluoxetine may indeed improve motor recovery of motor function-in patients with severe motor impairment. The AFFINITY trial did not include a large number patients with severe hemiparesis and did not measure motor recovery by the FMMS, but did measure motor functions as domains within the SIS and found no effect of fluoxetine. Our planned individual patient data meta-analysis of the FOCUS, AFFINITY and EFFECTS trials ${ }^{8}$ will constitute a larger number of stroke patients with severe motor impairments and promises to enable a more reliable analysis of the effect of fluoxetine, vs placebo, on the mRS and motor domains of the SIS at 6 months in this subgroup.

The AFFINITY trial also confirms the FOCUS trial finding that long-term fluoxetine in stroke patients has hazards, increasing the risk of bone fractures. ${ }^{9}$ We also found that fluoxetine significantly increased the risk of falls with injury and epileptic seizures in stroke patients. These hazards of fluoxetine were sought apriori during patient follow up. ${ }^{7,8}$-The FOCUS trial reported similar, but not significant, increases in falls with injury and seizures in patients allocated fluoxetine. ${ }^{9}$ The AFFINITY and FOCUS trials collectively provide robust evidence about the effect of an SSRI on the incidence of falls causing injury, and fractures, increasing the absolute risk of each by about $2 \%$ over 6 months among patients with recent stroke.

Although fluoxetine is more effective than placebo in treating major depressive disorders, ${ }^{10}$ and reduced the rate of new depression in the FOCUS, ${ }^{9}$ and other trials, ${ }^{27,29}$ we observed only improved mood and emotional control, as measured by the SIS, at 6 months with fluoxetine; 
the numerically lower rate of post-stroke depression with fluoxetine vs placebo was not statistically significant. We believe we lacked statistical power to show a significant effect of fluoxetine on post-stroke depression because the absolute rates of depression in both groups in AFFINITY were substantially lower (less than half) than in FOCUS, possibly from underreporting, particularly in Vietnam where the reporting of changes in mood may be affected by the cultural setting. ${ }^{30}$

In summary, the AFFINITY trial reinforces the conclusion of a recent Cochrane review that SSRIs are not effective at improving functional recoveryoutcome after stroke. It also confirms that fluoxetine may improve mood but have important adverse effects, particularly bone fractures. A planned individual patient data meta-analysis of the AFFINITY, FOCUS and EFFECTS trials will produce greater precision in the estimates of the effects of fluoxetine on recoveryfunctional outcome in important patient subgroups. ${ }^{8}$ 


\section{References}

1. Hankey GJ. Stroke. Lancet. 2017; 389: 641-654.

2. GBD 2016 Stroke Collaborators. Global, regional, and national burden of stroke, 1990-2016: a systematic analysis for the Global Burden of Disease Study 2016. Lancet Neurol. 2019; 18: 439-458.

3. Lim CM, Kim SW, Park JY, Kim C, Yoon SH, Lee JK. Fluoxetine affords robust neuroprotection in the post-ischemic brain via its anti-inflammatory effect. J Neurosci Res. 2009; 87: 1037-45.

4. Wang JW, David DJ, Monckton JE, Battaglia F, Hen R. Chronic fluoxetine stimulates maturation and synaptic plasticity of adult-born hippocampal granule cells. J Neurosci. 2008; 28:1374-84.

5. Chollet F, Tardy J, Albucher JF, et al. Fluoxetine for motor recovery after acute ischaemic stroke (FLAME): a randomised placebo-controlled trial. Lancet Neurol 2011; 10:123-30.

6. Mead GE, Hsieh CF, Lee R, et al. Selective serotonin reuptake inhibitors (SSRIs) for stroke recovery. Cochrane Database Syst Rev 2012; 11: CD009286.

7. Mead G, Hackett ML, Lundstrom E, Murray V, Hankey GJ, Dennis M. The FOCUS, AFFINITY and EFFECTS trials studying the effect(s) of fluoxetine in patients with a recent stroke: a study protocol for three multicentre randomised controlled trials. Trials 2015; 16: 369.

8. Graham C, Lewis S, Forbes J, et al. The FOCUS, AFFINITY and EFFECTS trials studying the effect(s) of fluoxetine in patients with a recent stroke: statistical and health economic analysis plan for the trials and for the individual patient data metaanalysis. Trials 2017; 18: 627.

9. FOCUS Trial Collaboration. Effects of fluoxetine on functional outcomes after acute stroke (FOCUS): a pragmatic, double-blind, randomised, controlled trial. Lancet. 2019; 393: 265-274.

10. Cipriani A, Furukawa TA, Salanti G, et al. Comparative efficacy and acceptability of 21 antidepressant drugs for the acute treatment of adults with major depressive disorder: a systematic review and network meta-analysis. Lancet. 2018; 39:13571366. 
11. Coupland C, Dhiman P, Morriss R, Arthur A, Barton G, Hippisley-Cox J. Antidepressant use and risk of adverse outcomes in older people: population based cohort study. BMJ. 2011; 343: d4551.

12. Brännström J, Lövheim H, Gustafson Y, Nordström P. Association between antidepressant drug use and hip fracture in older people before and after treatment initiation. JAMA Psychiatry. 2019; 76: 172-179.

13. Lundström E, Isaksson E, Näsman P, et al., Efficacy of fluoxetine - a randomised controlled trial in stroke (EFFECTS). The Lancet Neurology (?in press THELANCETNEUROLOGY-D-20-00292)

14. Altman DG, Bland JM. Treatment allocation by minimisation. BMJ 2005; 330: 843.

15. Counsell C, Dennis M, McDowall M, Warlow C. Predicting outcome after acute and subacute stroke: development and validation of new prognostic models. Stroke. 2002; 33: $1041-1047$

16. Bruno A, Shah N, Lin C, et al. Improving modified Rankin scale assessment with a simplified questionnaire. Stroke. 2010; 41: 1048-1050.

17. Williams LS, Brizendine EJ, Plue L, Bakas T, Tu W, Hendrie H, Kroenke K. Performance of the PHQ-9 as a screening tool for depression after stroke. Stroke. 2005; 36: 635-638.

18. de Jager CA, Budge MM, Clarke R. Utility of TICS-M for the assessment of cognitive function in older adults. International Journal of Geriatric Psychiatry. 2003; 18: $318-324$.

19. Duncan PW, Bode RK, Min Lai S, Perera S; Glycine Antagonist in Neuroprotection Americans Investigators. Rasch analysis of a new stroke-specific outcome scale: the Stroke Impact Scale. Arch Phys Med Rehabil. 2003; 84: 950-63.

20. Mead GE, Lynch J, Greig CA, Young A, Lewis S, Sharpe M. Evaluation of fatigue scales in stroke patients. Stroke. 2007; 38: 2090-2095.

21. Ware JE, Snow KK, Kosinski M, Gandek B. SF-36 health survey: manual and interpretation guide. Boston, MA: New England Medical Center, Health Institute; 1993.

22. Norman R, Cronin P, Viney R. A pilot discrete choice experiment to explore preferences for EQ-5D-5L health states. Appl Health Econ Health Policy. 2013; 11: 287-98. 
23. CLOTS Trials Collaboration. Thigh-length versus below-knee stockings for DVT prophylaxis after stroke: a randomized trial. Ann Int Med 2010; 153: 553-562.

24. IST-3 collaborative group. The benefits and harms of intravenous thrombolysis with recombinant tissue plasminogen activator within $6 \mathrm{~h}$ of acute ischaemic stroke (the third international stroke trial [IST-3]): a randomised controlled trial. Lancet 2012; 379: 2352-63.

25. Howard G, Waller JL, Voeks JH, et al.. A simple, assumption-free, and clinically interpretable approach for analysis of modified Rankin outcomes. Stroke. 2012; 43: 664-9.

26. Legg LA, Tilney R, Hsieh CF, et al. Selective serotonin reuptake inhibitors (SSRIs) for stroke recovery. Cochrane Database Syst Rev. 2019; 11: CD009286.pub3.

27. Mead GE, Legg L, Tilney R, et al. Fluoxetine for stroke recovery: Meta-analysis of randomized controlled trials. Int J Stroke. 2019 Oct 17. doi:

10.1177/1747493019879655. [Online ahead of print] PMID: 31619137 (accessed April 16, 2020).

28. van der Vliet R, Selles RW, Andrinopoulou ER, et al. Predicting Upper Limb Motor Impairment Recovery after Stroke: A Mixture Model. Ann Neurol. 2020; 87: 383393.

29. Kraglund KL, Mortensen JK, Damsbo AG, et al. Neuroregeneration and Vascular Protection by Citalopram in Acute Ischemic Stroke (TALOS). Stroke. 2018; 49: 2568-2576.

30. Steel Z, Silove D, Giao N, et al., International and indigenous diagnoses of mental disorder among Vietnamese living in Vietnam and Australia. Br J Psychiatry, 2009; 194: 326-333. 


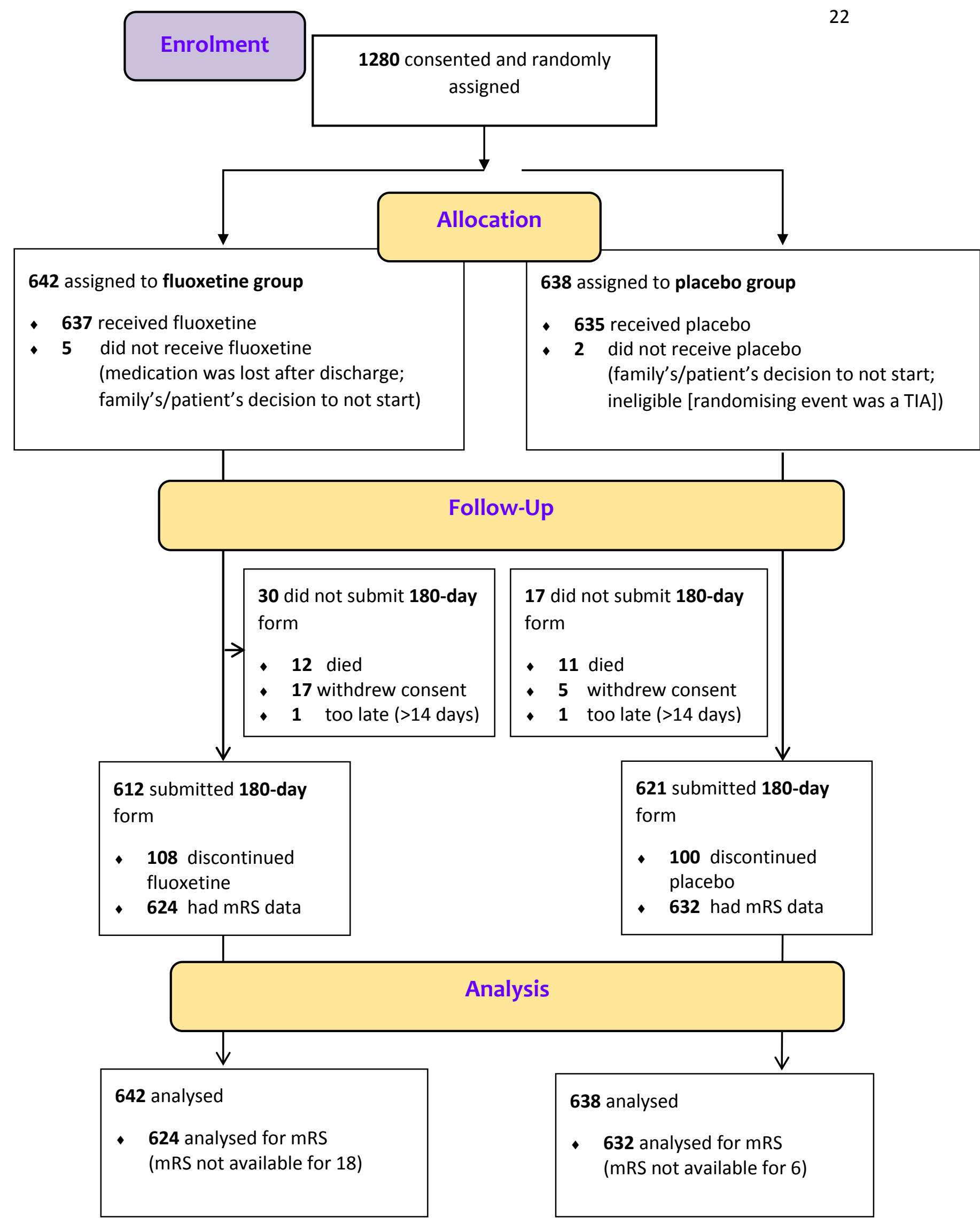

Figure 1: AFFINITY trial profile $m R S=$ modified Rankin Scale 


\section{Figure 2.}

\section{Primary outcome of the distribution of the modified Rankin Scale (mRS) scores at 6 months by treatment group.}

The primary outcome was an assessment of scores across all seven categories of the mRS (ranging from 0 [no symptoms] to 6 [death]), using a shift analysis of the ordinal data. The odds ratio and p-values were calculated with ordinal logistic regression, adjusted for the baseline variables included in the minimisation algorithm (delay between stroke onset and randomization, probability of being alive and independent at 6 months, presence of a motor deficit, presence of aphasia). mRS data at 6 months were available for 624 (97.2\%) patients allocated fluoxetine and $632(99.1 \%)$ allocated placebo. The common odds ratio was 0.936 (95\%CI: 0.762 to 1.150 ), $\mathrm{p}=0.53$; adjusted for baseline minimization variables. A common OR less than 1.0 favoured placebo.

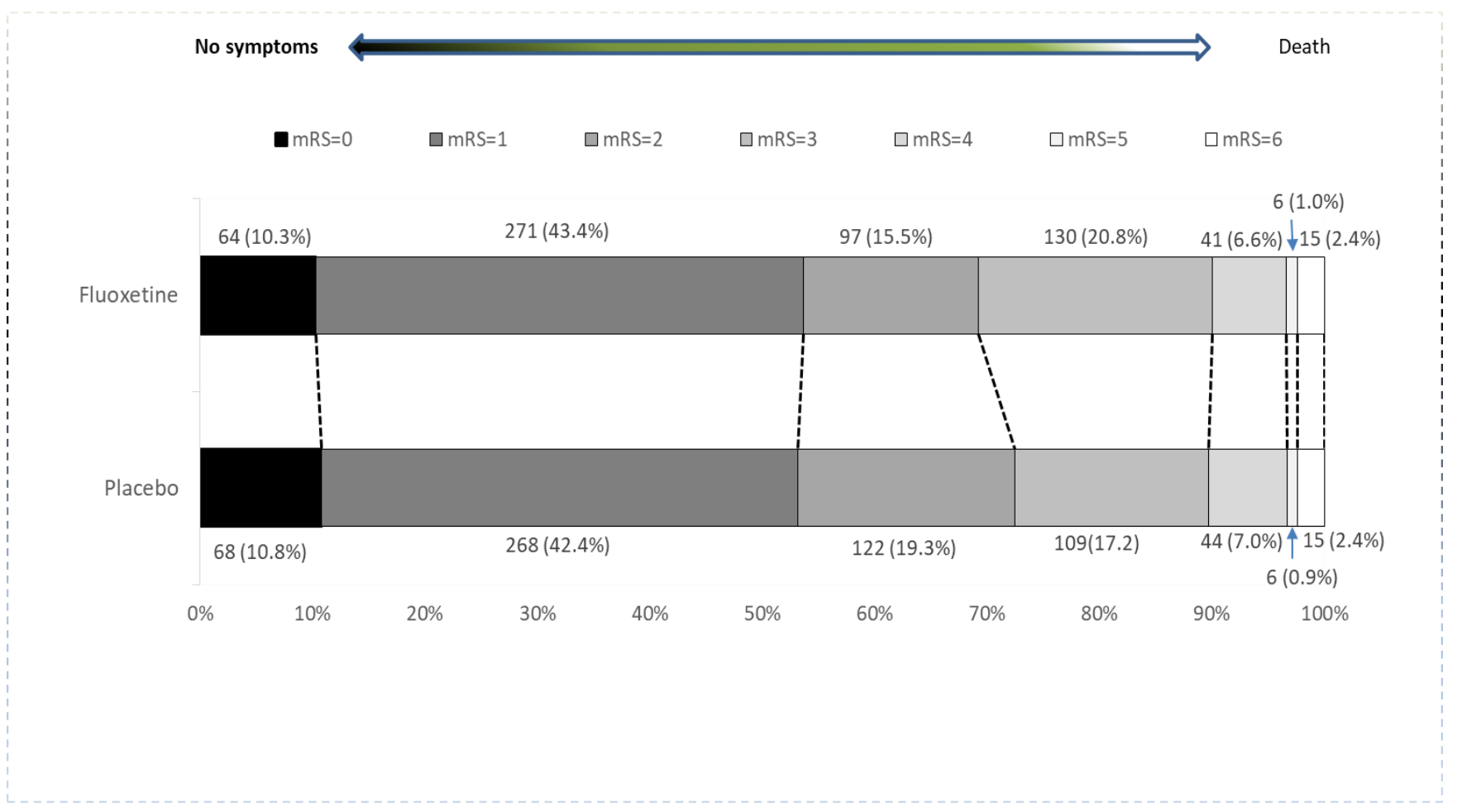




\section{Writing group of the AFFINITY trial Collaboration}

Graeme J. Hankey (Chair)

Professor of Neurology, Medical School, Faculty of Health and Medical Sciences, The University of Western Australia, Perth, Western Australia;

Neurologist, Department of Neurology Sir Charles Gairdner Hospital, Perth, Australia Maree L. Hackett

Professor, The George Institute for Global Health, Faculty of Medicine,

University of New South Wales Sydney;

Professor of Epidemiology, The University of Central Lancashire, United Kingdom.

Osvaldo P. Almeida

Professor of Geriatric Psychiatry, Medical School, Faculty of Health and Medical

Sciences, The University of Western Australia, Perth, Western Australia

Leon Flicker

Professor of Geriatric Medicine, Medical School, Faculty of Health and Medical Sciences, The University of Western Australia, Perth, Western Australia

Geriatrician, Royal Perth Hospital, Perth, Australia

Gillian E. Mead

Professor of Stroke and Elderly Care Medicine, University of Edinburgh, Royal Infirmary, Edinburgh

Martin S. Dennis

Professor of Stroke Medicine, University of Edinburgh, Royal Infirmary, Edinburgh Christopher Etherton-Beer

Associate Professor, The University of Western Australia, Perth, Western Australia Andrew H. Ford

Associate Professor, The University of Western Australia, Perth, Western Australia Laurent Billot

Director, Statistics Division, The George Institute for Global Health, New South Wales, Australia

Associate Professor, Faculty of Medicine, UNSW, Sydney

Stephen Jan

Professor of Health Economics, The George Institute for Global Health, Faculty of Medicine, University of New South Wales, Sydney, Australia

Thomas Lung

The George Institute for Global Health, Sydney

Erik Lundström

Associate Professor, Department of Neuroscience, Neurology, Uppsala University, Uppsala, Sweden. 
Craig S. Anderson

Professor of Neurology and Epidemiology, Faculty of Medicine, UNSW Sydney.

Neurologist, Neurology Department, Royal Prince Alfred Hospital.

The George Institute for Global Health at Peking University Health Science Center, Beijing, P.R.China.

The George Institute for Global Health, Sydney, Australia.

Huy Thang-Nguyen

Neurologist, The People's Hospital 115, Neurology, Ho Chi Min City, Vietnam John Gommans

Physician, Hawke's Bay Hospital, Hastings, New Zealand

Qilong Yi

Associate Professor, Canadian Blood Services and University of Toronto, Canada

\section{Contributors}

GJH was Chief Investigator A of the NHMRC Project Grant 1059094, Co-Chair of the steering committee, Chair of the trial coordinating committee, involved in the design of the trial, recruited and followed-up patients enrolled at his hospital site, adjudicated all adverse and serious adverse events in the trial (blind to treatment allocation), and wrote the first and final versions of the manuscript.

MLH was Chief Investigator B of the NHMRC Project Grant 1059094, Co-Chair of the steering committee, involved in the trial design, and advised on management of the trial.

OPA was Chief Investigator C of the NHMRC Project Grant 1059094, participated in the steering committee, involved in the trial design, and advised on the management of depression within the trial.

LF was Chief Investigator D of the NHMRC Project Grant 1059094, participated in the steering committee, and was involved in the trial design.

GEM was Chief Investigator E of the NHMRC Project Grant 1059094, participated in the steering committee as co-CI of the FOCUS trial, and was involved in the trial design.

MSD was Chief Investigator F of the NHMRC Project Grant 1059094, participated in the steering committee as co-CI of the FOCUS trial, was involved in the trial design, and advised regularly about trial management.

C E-B was Chief Investigator G of the NHMRC Project Grant 1059094 and participated in the steering committee.

AHF was Chief Investigator H of the NHMRC Project Grant 1059094, participated in the steering committee, and advised on the management of depression in the trial. 
LB was Chief Investigator I of the NHMRC Project Grant 1059094, participated in the steering committee, was involved in the trial design, advised on the statistical analysis plan and independently analysed the trial data and verified the final analysis.

SJ was Chief Investigator E of the NHMRC Project Grant 1059094 from 2014-2018, and participated in the steering committee.

TL was Chief Investigator E of the NHMRC Project Grant 1059094 in 2019.

VM (deceased December 2014) was an Associate Investigator of the NHMRC Project Grant 1059094, participated in the steering committee as CI of the EFFECTS trial, and was involved in the trial design.

EL participated in the steering committee as CI of the EFFECTS trial, and was involved in the trial design.

CSA was an Associate Investigator of the NHMRC Project Grant 1059094, participated in the steering committee, was involved in the trial design, and provided trial strategic advice.

JG was national coordinator for New Zealand, the Principal Investigator responsible for recruitment and follow-up of patients enrolled at his hospital site, and commented on the draft manuscript.

H T-N was national coordinator for Vietnam, and the Principal Investigator responsible for recruitment and follow-up of patients enrolled at his hospital site.

QY undertook the statistical analysis of the trial data for the Data Monitoring Committee meetings and for the final results.

The contributions other members of the AFFINITY trial collaboration are listed in the appendix (pp1-7).

\section{Declaration of interests}

Prof. Hankey reports grants from the National Health \& Medical Research Council of Australia, Vetenskapsrådet (The Swedish Research Council), and United Kingdom National Institute for Health Research Technology (NIHR), during the conduct of the study; and personal fees from American Heart Association, outside the submitted work.

Prof. Hackett reports grants from National Health and Medical Research Council during the conduct of the study.

Prof. Etherton-Beer reports grants from National Health and Medical Research Council (NHMRC) of Australia, during the conduct of the study.

Prof. Billot reports grants from NHMRC during the conduct of the study.

Dr. Lung reports grants from Australian National Health and Medical Research Council, during the conduct of the study. 
Prof. Anderson reports grants from National Health and Medical Research Council (NHMRC) of Australia, grants from Takeda, personal fees from Takeda, outside the submitted work.

Profs Almeida, Flicker, Mead, Dennis, Ford, Billot, Jan, Lundström, Thang-Nguyen, Gommans, and Yi report nothing to disclose.

\section{Data sharing}

The trial protocol and statistical analysis plan have been published. ${ }^{7,8}$ A fully anonymised trial dataset with individual patient data and a data dictionary will be available to other researchers after the publication of the full trial results from the final follow-up at 12 months. Written proposals and requests are to be directed to Graeme Hankey (Co-Chief Investigator). Proposals will be assessed by the AFFINITY trial Steering Committee and a data sharing agreement established if, and before, any data are to be shared.

\section{Acknowledgements}

The AFFINITY trial was funded by the Australian National Health and Medical Research Council (NHMRC) Project Grant 1059094. The minimisation algorithm was provided by 'The Stroke Research Group, Division of Clinical Neuroscience, The University of Edinburgh. The manufacture, storage and distribution of the trial medication was undertaken by Pharmaceutical Packaging Professionals Pty Ltd (Trading as PCI Pharma Services), an Australian Therapeutic Goods Administration-licenced Good Manufacturing Practices facility. We are grateful to all patients and their families who participated in AFFINITY, and the nursing staff who assisted at collaborating sites for contributing to this research endeavour. 
Table 1. Patient characteristics at randomization by allocated treatment

\begin{tabular}{|c|c|c|}
\hline & $\begin{array}{c}\text { Fluoxetine } \\
(n=642)\end{array}$ & $\begin{array}{l}\text { Placebo } \\
(n=638)\end{array}$ \\
\hline \multicolumn{3}{|l|}{ Sex } \\
\hline Women & $231(36 \%)$ & $245(38 \%)$ \\
\hline Men & $411(64 \%)$ & $393(62 \%)$ \\
\hline \multicolumn{3}{|l|}{ Age } \\
\hline Age $\leq 70$ years & $450(70 \%)$ & $432(68 \%)$ \\
\hline Age $>70$ years & $192(30 \%)$ & $206(32 \%)$ \\
\hline Mean age, years & $63.5(12.5)$ & $64.6(12.2)$ \\
\hline \multicolumn{3}{|l|}{ Ethnicity } \\
\hline Asian & $356(55 \%)$ & $371(58 \%)$ \\
\hline White & $267(42 \%)$ & $255(40 \%)$ \\
\hline Other & $19(3 \%)$ & $12(2 \%)$ \\
\hline \multicolumn{3}{|l|}{ Marital status } \\
\hline Married & $463(72 \%)$ & $463(73 \%)$ \\
\hline Partner & $29(4 \%)$ & $19(3 \%)$ \\
\hline Divorced or separated & $37(6 \%)$ & $46(7 \%)$ \\
\hline Widowed & $61(9 \%)$ & $70(11 \%)$ \\
\hline Single & $52(8 \%)$ & $38(6 \%)$ \\
\hline Other & $0(0 \%)$ & $2(0 \%)$ \\
\hline \multicolumn{3}{|l|}{ Living arrangements } \\
\hline Living with someone else & $564(88 \%)$ & $556(87 \%)$ \\
\hline Living alone & $78(12 \%)$ & $78(12 \%)$ \\
\hline Living in an institution & $0(0 \%)$ & $2(0 \%)$ \\
\hline Other & $0(0 \%)$ & $2(0 \%)$ \\
\hline \multicolumn{3}{|l|}{ Employment status } \\
\hline Full-time employment & $206(32 \%)$ & $180(28 \%)$ \\
\hline Part-time employment & $77(12 \%)$ & $68(11 \%)$ \\
\hline Retired & $315(49 \%)$ & $363(57 \%)$ \\
\hline Unemployed or disabled & $24(4 \%)$ & $14(2 \%)$ \\
\hline Other & $20(3 \%)$ & $13(2 \%)$ \\
\hline Independent before stroke & $634(99 \%)$ & $630(99 \%)$ \\
\hline \multicolumn{3}{|l|}{ Previous medical history } \\
\hline Coronary Heart Disease & $58(9 \%)$ & $57(9 \%)$ \\
\hline Ischaemic stroke or TIA & $77(12 \%)$ & $84(13 \%)$ \\
\hline Diabetes & $143(23 \%)$ & $147(23 \%)$ \\
\hline Hyponatraemia & $1(0 \%)$ & $3(0 \%)$ \\
\hline Intracranial bleed & $11(2 \%)$ & $8(1 \%)$ \\
\hline Upper gastrointestinal bleed & $11(2 \%)$ & $15(2 \%)$ \\
\hline Bone fractures & $71(11 \%)$ & $74(12 \%)$ \\
\hline Depression & $30(5 \%)$ & $20(3 \%)$ \\
\hline \multicolumn{3}{|l|}{ Stroke diagnosis } \\
\hline Non-stroke (final diagnosis) & $3(0 \%)$ & $1(0 \%)$ \\
\hline Ischaemic stroke & $549(86 \%)$ & $542(85 \%)$ \\
\hline
\end{tabular}




\begin{tabular}{|c|c|c|}
\hline Intracerebral haemorrhage & $90(14 \%)$ & $95(15 \%)$ \\
\hline \multicolumn{3}{|l|}{ OCSP classification of Ischaemic stroke } \\
\hline Total anterior circulation infarct & $47(9 \%)$ & $50(9 \%)$ \\
\hline Partial anterior circulation infarct & $271(49 \%)$ & $283(52 \%)$ \\
\hline Lacunar infarct & $115(21 \%)$ & $105(19 \%)$ \\
\hline Posterior circulation infarct & $114(21 \%)$ & $103(19 \%)$ \\
\hline Uncertain & $2(0 \%)$ & $1(0 \%)$ \\
\hline \multicolumn{3}{|l|}{$\begin{array}{l}\text { Causes of ischaemic stroke (modified } \\
\text { TOAST classification) }\end{array}$} \\
\hline Large artery disease & $123(22 \%)$ & $134(25 \%)$ \\
\hline Small vessel disease & $261(47 \%)$ & $250(46 \%)$ \\
\hline Embolism from the heart & $95(17 \%)$ & $93(17 \%)$ \\
\hline Another cause & $9(2 \%)$ & $8(1 \%)$ \\
\hline Unknown or uncertain cause & $61(11 \%)$ & $57(10 \%)$ \\
\hline \multicolumn{3}{|l|}{ Predictive variables } \\
\hline Able to walk at time of randomisation & $282(44 \%)$ & $279(44 \%)$ \\
\hline Able to lift both arms off bed & $443(69 \%)$ & $431(68 \%)$ \\
\hline Able to talk and not confused & $554(86 \%)$ & $557(87 \%)$ \\
\hline \multicolumn{3}{|l|}{$\begin{array}{l}\text { Predicted 6-month outcome based on } \\
\text { SSV }\end{array}$} \\
\hline $\begin{array}{l}\text { Probability of being alive and } \\
\text { independent }\end{array}$ & $\begin{array}{c}0.57 \\
(0.26-0.87) \\
\end{array}$ & $\begin{array}{c}0.55 \\
(0.24-0.87) \\
\end{array}$ \\
\hline 0.00 to $\leq 0.15$ & $100(15 \%)$ & $103(16 \%)$ \\
\hline 0.15 to 1.00 & $542(84 \%)$ & $535(84 \%)$ \\
\hline \multicolumn{3}{|l|}{ Neurological deficits } \\
\hline NIHSS & $6(3-9)$ & $6-(3-9)$ \\
\hline Presence of a motor deficit & $557(87 \%)$ & $548(86 \%)$ \\
\hline Presence of aphasia & $129(20 \%)$ & $121(19 \%)$ \\
\hline \multicolumn{3}{|l|}{ Depression at baseline } \\
\hline $\begin{array}{l}\text { Current diagnosis of depression (patient } \\
\text { or proxy reported) }\end{array}$ & $15(2 \%)$ & $17(3 \%)$ \\
\hline Taking a non-SSRI antidepressant & $5(1 \%)$ & $5(1 \%)$ \\
\hline \multicolumn{3}{|l|}{ Current mood } \\
\hline PHQ-9, median (IQR) & $4(1-7)$ & $4(2-7)$ \\
\hline $0-14$ & $601(98 \%)$ & $596(98 \%)$ \\
\hline$\geq 15$ & $12(2 \%)$ & $11(2 \%)$ \\
\hline \multicolumn{3}{|l|}{$\begin{array}{l}\text { Delay (days) since stroke onset at } \\
\text { randomisation }\end{array}$} \\
\hline Mean delay & $6.1(3 \%)$ & $6.3(3 \%)$ \\
\hline 2-8 days & $486(76 \%)$ & $479(75 \%)$ \\
\hline 9-15 days & $156(24 \%)$ & $159(25 \%)$ \\
\hline \multicolumn{3}{|l|}{ Consent } \\
\hline Patient consented & $345(54 \%)$ & $328(51 \%)$ \\
\hline Person responsible consented & $284(44 \%)$ & $295(46 \%)$ \\
\hline Proxy consented & $3(0 \%)$ & $1(0 \%)$ \\
\hline Waiver acknowledgement & $10(2 \%)$ & $14(2 \%)$ \\
\hline
\end{tabular}


Data are $\mathrm{n}(\%)$, mean (SD [standard deviation]), or median (IQR [interquartile range]) TIA: transient ischaemic attack

OCSP $=$ Oxfordshire Community Stroke Project

TOAST $=$ modified Trial of ORG 10172 in acute stroke treatment criteria

$\mathrm{SSV}=$ Six simple variables the predict functional outcome, as measured by the mRS, after stroke (age, living alone before the stroke, independent in activities of daily living before the stroke, and able to talk, lift both arms off the bed, and walk unassisted at the time of randomisation). ${ }^{15}$

NIHSS = National Institutes of Health Research Stroke Scale

SSRI: selective serotonin reuptake inhibitor

PHQ-9 = Patient Health Questionnaire 9 items (higher scores indicate more depressive symptoms). ${ }^{17}$ 
Table 2. Secondary outcomes at six months by allocated treatment

\begin{tabular}{|l|c|c|c|c|c|}
\hline \multicolumn{2}{|c|}{ Fluoxetine (n=642) } & \multicolumn{2}{|c|}{ Placebo (n=638) } & P value \\
\hline $\begin{array}{l}\text { New depression } \\
\text { N / N (\%) }\end{array}$ & $33(5.1 \%)$ & & $46(7.2 \%)$ & & 0.13 \\
\hline Mood (PHQ-9) & 2.0 & $(1.0-5.0)$ & 2.0 & $(1.0-5.0)$ & 0.42 \\
\hline $\begin{array}{l}\text { PHQ-9 } \geq 15 \\
\text { N / N\% }\end{array}$ & $4(0.7 \%)$ & & $6(1.0 \%)$ & & 0.75 \\
\hline Cognition (TICSm) & 24.0 & $(20.0-27.0)$ & 24.0 & $(19.0-27.0)$ & 0.62 \\
\hline $\begin{array}{l}\text { Stroke Impact Scale } \\
\text { SIS) domains }\end{array}$ & & & & & \\
\hline -Strength & 75.0 & $(56.3-93.8)$ & 75.0 & $(56.3-93.8)$ & 0.26 \\
\hline -Hand ability & 85.0 & $(55.0-100.0)$ & 85.0 & $(55.0-100.0)$ & 0.39 \\
\hline -Mobility & 91.7 & $(69.4-100.0)$ & 88.9 & $(66.7-97.2)$ & 0.08 \\
\hline -Motor† & 83.5 & $(63.5-94.2)$ & 82.4 & $(60.4-93.2)$ & 0.28 \\
\hline -Daily Activities & 90.0 & $(72.5-100.0)$ & 90.0 & $(70.0-97.5)$ & 0.25 \\
\hline -Physical function† & 85.5 & $(66.2-94.9)$ & 83.8 & $(63.4-93.8)$ & 0.24 \\
\hline -Memory & 89.3 & $(78.6-100.0)$ & 89.3 & $(75.0-100.0)$ & 0.28 \\
\hline -Communication & 98.2 & $(89.3-100.0)$ & 96.4 & $(85.7-100.0)$ & 0.61 \\
\hline -Mood/Emotions^ & 80.6 & $(66.7-88.9)$ & 77.8 & $(66.7-86.1)$ & 0.003 \\
\hline -Participation & 81.3 & $(59.4-96.9)$ & 75.0 & $(56.3-96.9)$ & 0.48 \\
\hline -Recovery (VAS) & 80.0 & $(60.0-90.0)$ & 80.0 & $(60.0-90.0)$ & 0.90 \\
\hline Vitality (SF-36) & 70.0 & $(55.0-80.0)$ & 70.0 & $(55.0-80.0)$ & 0.36 \\
\hline EQ5D-5L & 0.81 & $0.63-1.00$ & 0.78 & $0.58-0.93$ & 0.08 \\
\hline
\end{tabular}

Data were only available for those who survived and who completed sufficient questions to derive a score. The number of patients with missing scores were similar in the two treatment groups.

Data are median (IQR).

PHQ-9: Patient Health Questionnaire 9 items (higher score indicates more depressive symptoms)

TICSm: Telephone Interview for Cognitive Status

SIS: Stroke Impact Scale (where higher scores are better).

$\dagger$ Mean of the Strength, Hand ability, and Mobility domains.

$\$$ Mean of the Strength, Hand ability, Mobility, and Daily activities domains.

${ }^{\wedge}$ Mood/Emotions domain of the SIS: Nine questions about "how you feel, changes in your mood, and your ability to control your emotions, since your stroke." (where higher scores are better)

VAS: visual analogue scale.

SF-36: 36 item short form questionnaire (higher scores indicate less disability) 
EQ5D-5L: EuroQoL - 5 Dimensions (Mobility, Personal Care, Usual Activities, Pain/Discomfort, Anxiety/Depression) - 5 Levels (where 1 indicates the best health imaginable, and -0.676 indicates the worst health imaginable). 
Table 3. Adverse events at 6 months by allocated treatment group

\begin{tabular}{|c|c|c|c|c|}
\hline & $\begin{array}{c}\text { Fluoxetine } \\
(\mathrm{n}=642)\end{array}$ & $\begin{array}{l}\text { Placebo } \\
(n=638)\end{array}$ & Difference $(95 \% \text { CI })^{*}$ & $\begin{array}{c}\text { P- } \\
\text { value }\end{array}$ \\
\hline Death & $15(2.34 \%)$ & $15(2.35 \%)$ & $=0.01 \%(-1.64$ to 1.67$)$ & 1.00 \\
\hline Any stroke & $18(2.80 \%)$ & $26(4.08 \%)$ & $-1.27 \%(-3.27$ to 0.72$)$ & 0.22 \\
\hline \multicolumn{5}{|l|}{ All thrombotic events } \\
\hline Ischaemic stroke & $11(1.71 \%)$ & $21(3.29 \%)$ & $-1.58 \%(-3.29$ to 0.13$)$ & 0.08 \\
\hline Acute coronary events & $1(0.16 \%)$ & $2(0.31 \%)$ & $-0.16 \%(-0.69$ to 0.37$)$ & 0.62 \\
\hline \multicolumn{5}{|l|}{ All bleeding events } \\
\hline Haemorrhagic stroke & $3(0.47 \%)$ & $1(0.16 \%)$ & $0.31 \%(-0.30$ to 0.92$)$ & 0.62 \\
\hline Upper gastrointestinal bleed & $1(0.16 \%)$ & $1(0.16 \%)$ & $0.00 \%(-0.43$ to 0.43$)$ & 1.00 \\
\hline Epileptic seizures & $10(1.56 \%)$ & $2(0.31 \%)$ & $1.24 \%(0.19$ to 2.30$)$ & 0.04 \\
\hline Fall with injury & $20(3.12 \%)$ & $7(1.10 \%)$ & $2.02 \%(0.45$ to 3.59$)$ & 0.02 \\
\hline New bone fracture & $19(2.96 \%)$ & $6(0.94 \%)$ & $2.02 \%(0.51$ to 3.53$)$ & 0.01 \\
\hline Hyponatraemia $<125 \mathrm{mmol} / \mathrm{l}$ & $3(0.47 \%)$ & $2(0.31 \%)$ & $0.15 \%(-0.53$ to 0.84$)$ & 1.00 \\
\hline Hyperglycaemia & $0(0 \%)$ & $0(0 \%)$ & $0 \%$ & \\
\hline Symptomatic hypoglycaemia & $0(0 \%)$ & $0(0 \%)$ & $0 \%$ & \\
\hline New depression & $33(5.14 \%)$ & $46(7.21 \%)$ & $-2.07 \%(-4.71$ to 0.57$)$ & 0.13 \\
\hline New antidepressant & $30(4.67 \%)$ & $43(6.74 \%)$ & $-2.07 \%(-4.61$ to 0.47$)$ & 0.12 \\
\hline Attempted or actual suicide & $0(0 \%)$ & $2(0.31 \%)$ & $-0.16 \%(-0.75$ to 0.12$)$ & 0.25 \\
\hline Other adverse event & $62(9.66 \%)$ & $68(10.66 \%)$ & $-1.00 \%(-4.31$ to 2.31$)$ & 0.56 \\
\hline
\end{tabular}

Data are n (\%), unless otherwise stated

* Risk differences and their 95\%confidence intervals were calculated in SAS by means of the FREQ procedure.

https://documentation.sas.com/?docsetId=procstat\&docsetTarget=procstat_freq_detail $\underline{\text { s54.htm \&docsetVersion }=9.4 \& \text { locale }=\text { en }}$ (accessed March 18, 2020). The confidence intervals around the risk differences are Wald intervals based on the normal approximation. 
ASSESSMENT OE FLUOXETINE IN STROKE RECOVERY (AFFINITY) TRIAL

Manuscript Number: THELANCETNEUROLOGY-D-20-00293

\begin{tabular}{|c|c|}
\hline First/Middle Name & Surname \\
\hline Graeme J. & Hankey \\
\hline Maree L. & Hackett \\
\hline Osvaldo P. & Almeida \\
\hline Leon & Flicker \\
\hline Gillian E. & Mead \\
\hline Martin S. & Dennis \\
\hline Christopher & Etherton-Beer \\
\hline Andrew $\mathrm{H}$. & Ford \\
\hline Laurent & Billot \\
\hline Stephen & Jan \\
\hline Thomas & Lung \\
\hline Veronica & Murray \\
\hline Erik & Lundström \\
\hline Craig S. & Anderson \\
\hline Robert & Herbert \\
\hline Gregory & Carter \\
\hline Geoffrey A. & Donnan \\
\hline Huy & Thang-Nguyen \\
\hline John & Gommans \\
\hline Qilong & $\mathrm{Yi}$ \\
\hline Qiang & $\mathrm{Li}$ \\
\hline Severine & Bompoint \\
\hline Sarah & Barrett \\
\hline Anne & Claxton \\
\hline Julia & O'Dea \\
\hline Michelle & Tang \\
\hline Clare & Williams \\
\hline Shenae & Peterson \\
\hline Christie & Drummond \\
\hline Uyen & Ha-Hong \\
\hline Linh & Thi My-Le \\
\hline Tram & Thi Bich-Ngo \\
\hline Yen & Bao-Mai \\
\hline Huyen & Thanh-Han \\
\hline Nhu & Quynh-Truong \\
\hline Huong & Thi-Nguyen \\
\hline Hai & Thanh-Ngo \\
\hline Thuan & Thi Binh-Nguyen \\
\hline Oanh & Thi Kieu-Ha \\
\hline Trang & Le Huyen-Nguyen \\
\hline Richard I. & Lindley \\
\hline Peter & New \\
\hline Andrew & Lee \\
\hline Thanh & Trung Tran \\
\hline Thuy & Le Vu-Kieu \\
\hline Loan & Tran Truc Mai-Le \\
\hline Sang & Van-Nguyen \\
\hline Thuy & Anh Diem-Nguyen \\
\hline
\end{tabular}




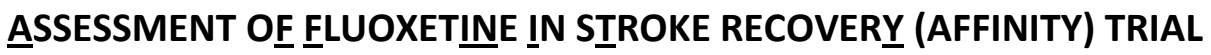

Manuscript Number: THELANCETNEUROLOGY-D-20-00293

\begin{tabular}{|c|c|}
\hline Tam & Nhat-Dang \\
\hline Hanh & Thi Truc-Phan \\
\hline Loan & Thi Ngoc-Vo \\
\hline Mai & Hue-Nguyen \\
\hline Hanh & Cao-Dang \\
\hline Hong & Thi-Tran \\
\hline Linh & Thi Cam-Dam \\
\hline Trinh & Thi Kim-Ngo \\
\hline Binh & Nguyen-Pham \\
\hline Nha & Thi Thanh-Dao \\
\hline Huong & Thi Bich-Nguyen \\
\hline Linh & Thi Cam-Le \\
\hline Chi & Minh-Do \\
\hline Thai & Nguyen Thanh-Pham \\
\hline Huy & Quoc-Huynh \\
\hline Giau & Thi Kim-Tran \\
\hline Oanh & Thi-Le \\
\hline Ly & Thi Khanh-Tran \\
\hline Chinh & Dinh - Duong \\
\hline Hoa & Ngoc - Nguyen \\
\hline Long & Van - Nguyen \\
\hline Binh & Van - Le \\
\hline Duong & Van - Kieu \\
\hline Long & Thanh - Nguyen \\
\hline $\mathrm{Na}$ & Le \\
\hline Tuan & Quoc - Tuan \\
\hline Tan & Van - Vo \\
\hline Khanh & Kim-Le \\
\hline Tram & Ngoc-Bui \\
\hline Nga & Thuy - Lam \\
\hline Phuong & Thanh - Trinh \\
\hline $\mathrm{Ha}$ & Thi Thu - Nguyen \\
\hline Uyen & Thi To - Hoang \\
\hline Hien & Thi Bich - Nguyen \\
\hline Tham & Hong - Pham \\
\hline Hop & Quang - Huynh \\
\hline Thao & Thi Thu - Nguyen \\
\hline Huyen & Ngoc-Lu \\
\hline Sam & Hoanh - Nguyen \\
\hline Sung & Phuoc - Pham \\
\hline Thuc & Van-Tran \\
\hline Bich & Thi - Doan \\
\hline Ninh & Hong - Le \\
\hline Giang & Truong - Nguyen \\
\hline Duong & Huu - Luong \\
\hline $\mathrm{Ha}$ & Van - Tran \\
\hline Phuong & \begin{tabular}{|l|} 
Thi - Do \\
\end{tabular} \\
\hline Hoai & Thi - Le \\
\hline Chi & Van-Nguyen \\
\hline Phuong & Doan-Nguyen \\
\hline
\end{tabular}




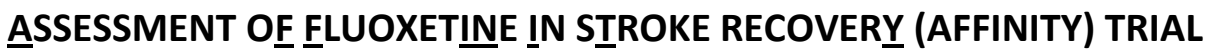

Manuscript Number: THELANCETNEUROLOGY-D-20-00293

\begin{tabular}{|c|c|}
\hline Ton & \begin{tabular}{|l} 
Duy-Mai \\
\end{tabular} \\
\hline Phuong & Viet-Dao \\
\hline Dung & Tien-Nguyen \\
\hline Dai & Quoc-Khuong \\
\hline Trung & Xuan-Vuong \\
\hline Lan & Tuong -Vu \\
\hline Ngoc & Duc-Ngo \\
\hline Hanh & Hong-Dang \\
\hline Phuong & Thai-Truong \\
\hline Ngan & Thi-Le \\
\hline Hoa & Van-Hoang \\
\hline Chung & Quang-Do \\
\hline Minh & Thao-Nguyen \\
\hline Anh & Hai-Dam \\
\hline Quynh & \begin{tabular}{|l|} 
Nhu-Le \\
\end{tabular} \\
\hline Ngoc & Hoang-Nguyen \\
\hline Toan & Dinh-Le \\
\hline Tuyen & Van-Nguyen \\
\hline Loan & Thi-Nguyen \\
\hline $\mathrm{Ha}$ & Thi Hai-Dinh \\
\hline Khanh & Thi Ngoc-Thach \\
\hline Cuong & Van-Pham \\
\hline Linh & Hai-Nguyen \\
\hline Phuong & Hong-Tran \\
\hline Vien & Chi - Le \\
\hline Tai & Anh - Nguyen \\
\hline Tuan & Van-Le \\
\hline Luyen & Van - Truong \\
\hline Tue & Chau-Bui \\
\hline Ngoc & Xuan - Huynh \\
\hline Lap & Van-Dinh \\
\hline An & Gia-Pham \\
\hline Trang & Thi Huyen - Le \\
\hline Vy & Tuong - Nguyen \\
\hline Yen & Hai - Nguyen \\
\hline Thang & Ba - Nguyen \\
\hline Huy & Thai \\
\hline Quyen & Thi Ngoc-Pham \\
\hline Khoa & Duy - Dao \\
\hline Quoc & Nguyen Bao - Pham \\
\hline Thuong & Thi Huyen - Dang \\
\hline Huong & Huynh To - Dinh \\
\hline Trang & Mai-Tong \\
\hline Thuy & Thi - Vu \\
\hline $\mathrm{Si}$ & Tri-Le \\
\hline Tai & Ngoc-Tran \\
\hline Phuong & Hoai - Tran \\
\hline Ngoc & Thuy Nhu - Dinh \\
\hline Binh & Thanh-Nguyen \\
\hline Vinh & Phuong-Do \\
\hline
\end{tabular}




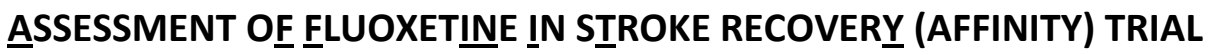

Manuscript Number: THELANCETNEUROLOGY-D-20-00293

\begin{tabular}{|c|c|}
\hline Anh & Ngoc-Nguyen \\
\hline Binh & Thi Thanh-Nguyen \\
\hline Binh & Thanh-Nguyen \\
\hline David & Blacker \\
\hline Anne & Claxton \\
\hline Lindsey & Bunce \\
\hline Ai Ling & Tan \\
\hline Darshan & Ghia \\
\hline Gillian & Edmonds \\
\hline Nicole & O'Loughlin \\
\hline Megan & Ewing \\
\hline Kerri-Ann & Whittaker \\
\hline Lorralee & Deane \\
\hline Darshan & Ghia \\
\hline Graeme & Hankey \\
\hline Anne & Claxton \\
\hline Yash & Gawarikar \\
\hline Brett & Jones \\
\hline Maria & Lopez \\
\hline Koushik & Nagesh \\
\hline Emma & Siracusa \\
\hline Stephen & Davis \\
\hline Amy & McDonald \\
\hline Jess & Tsoleridis \\
\hline Rachael & McCoy \\
\hline David & Jackson \\
\hline Gab & Silver \\
\hline Tim & Bates \\
\hline Amanda & Boudville \\
\hline Lynda & Southwell \\
\hline Dennis & \begin{tabular}{|l|} 
Cordato \\
\end{tabular} \\
\hline Alan J. & McDougall \\
\hline Cecilia & Cappelen-Smith \\
\hline Zeljka & Calic \\
\hline Shabeel & Askar \\
\hline Qi & Cheng \\
\hline Raymond & Kumar \\
\hline Richard & Geraghty \\
\hline Maree & Duroux \\
\hline Megan & Ratcliffe \\
\hline Samantha & Shone \\
\hline Cassandra & McLennan \\
\hline Ramesh & Sahathevan \\
\hline Casey & Hair \\
\hline Stanley & Levy \\
\hline Beverley & Macdonald \\
\hline Benjamin & Nham \\
\hline Louise & Rigney \\
\hline Dev & Nathani \\
\hline
\end{tabular}


ASSESSMENT OE FLUOXETINE IN SIROKE RECOVER్ (AFFINITY) TRIAL

Manuscript Number: THELANCETNEUROLOGY-D-20-00293

\begin{tabular}{|c|c|}
\hline Sumana & Gopinath \\
\hline Vishal & Patel \\
\hline Abul & Mamun \\
\hline Benjamin & Trewin \\
\hline Chun & Phua \\
\hline $\mathrm{Ho}$ & Choong \\
\hline Lauren & Tarrant \\
\hline Kerry & Boyle \\
\hline Luisa & Hewitt \\
\hline Monique & Hourn \\
\hline Amanda & Masterson \\
\hline Kim & Oakley \\
\hline Karen & Ruddell \\
\hline Colette & Sanctuary \\
\hline Kimberley & Veitch \\
\hline Camelia & Burdusel \\
\hline Lina & Lee \\
\hline Gary & Cheuk \\
\hline Jeremy & Christley \\
\hline Tabitha & Hartwell \\
\hline Craig & Davenport \\
\hline Kate & Hickey \\
\hline Rosanna & Robertson \\
\hline Michelle & Carr \\
\hline Sam & Akbari \\
\hline Hannah & Coyle \\
\hline Megan & O'Neill \\
\hline Cameron & Redpath \\
\hline Caroline & Roberts \\
\hline Marjan & Tabesh \\
\hline Toni & \begin{tabular}{|l|} 
Withiel \\
\end{tabular} \\
\hline Kapila & Abeysuriya \\
\hline Andrew & Granger \\
\hline Angela & Abraham \\
\hline Chermaine & Chua \\
\hline Dung & Do Nguyen \\
\hline Vathani & Surendran \\
\hline Melissa & Daines \\
\hline David & Shivlal \\
\hline Mudassar & Latif \\
\hline Noreen & Mughal \\
\hline Patricia & Morgan \\
\hline Martin & Krause \\
\hline Miriam & Priglinger \\
\hline Ehsan E. & Shandiz \\
\hline Susan & Day \\
\hline Tim & Bates \\
\hline Lay & Kho \\
\hline
\end{tabular}


ASSESSMENT OE FLUOXETINE IN SIROKE RECOVER్ (AFFINITY) TRIAL

Manuscript Number: THELANCETNEUROLOGY-D-20-00293

\begin{tabular}{|c|c|}
\hline Michael & Pollack \\
\hline Judith & Dunne \\
\hline Helen & Baines \\
\hline Merridie & Rees \\
\hline Jenni & White \\
\hline Monique & Hourn \\
\hline Kimberley & Veitch \\
\hline Aicuratiya & Withanage \\
\hline Colette & Sanctuary \\
\hline Candice & Delcourt \\
\hline Cheryl & Carcel \\
\hline Alejandra & Malavera \\
\hline Amy & Kunchok \\
\hline Elizabeth & Ray \\
\hline Elizabeth & Pepper \\
\hline Emily & Duckett \\
\hline Jenni & White \\
\hline Kimberley & Veitch \\
\hline Luisa & Hewitt \\
\hline Monique & Hourn \\
\hline Kerry & Boyle \\
\hline Sally & Ormond \\
\hline Colette & Sanctuary \\
\hline Andrew & Moey \\
\hline Timothy & Kleinig \\
\hline Vanessa & Maxwell \\
\hline Chantal & Baldwin \\
\hline Wilson & Vallat \\
\hline Deborah & Field \\
\hline Romesh & Markus \\
\hline Kirsty & Page \\
\hline Danielle & Wheelwright \\
\hline Sam & Bolitho \\
\hline Steven & Faux \\
\hline Fix & Sangvatanakul \\
\hline Alexis & Brown \\
\hline Susan & Walker \\
\hline Jennifer & Massey \\
\hline Michael & Pollack \\
\hline Jenni & White \\
\hline Kimberley & Veitch \\
\hline Hillary & Hayes \\
\hline Luisa & Hewitt \\
\hline Monique & Hourn \\
\hline Colette & Sanctuary \\
\hline Pesi & Katrak \\
\hline Annie & Winker \\
\hline Alessandro & Zagami \\
\hline Alanah & Bailey \\
\hline
\end{tabular}


ASSESSMENT OE FLUOXETINE IN SIROKE RECOVER్ (AFFINITY) TRIAL

Manuscript Number: THELANCETNEUROLOGY-D-20-00293

\begin{tabular}{|c|c|}
\hline Sarah & Mccormack \\
\hline Andrew & Murray \\
\hline Mark & Rollason \\
\hline Christopher & Taylor \\
\hline Fintan & O'Rourke \\
\hline Ye Min & Kuang \\
\hline Heike & Burnet \\
\hline Yvonne & Liu \\
\hline Qi & Cheng \\
\hline Aileen & $\mathrm{Wu}$ \\
\hline Sam & Akbari \\
\hline Hannah & Coyle \\
\hline Megan & O'Neill \\
\hline Diana & Ramirez \\
\hline Tissa & Wijeratne \\
\hline Sherisse & Celestino \\
\hline Essie & Low \\
\hline Cynthia & Chen \\
\hline Jennifer & Bergqvist \\
\hline Andrew & Evans \\
\hline Queenie & Leung \\
\hline Martin & Jude \\
\hline Rachael & McQueen \\
\hline Katherine & Mohr \\
\hline Latitia & Kernaghan \\
\hline Paul & Stockle \\
\hline Boon L. & Tan \\
\hline Sara & Laubscher \\
\hline Diana & Schmid \\
\hline Melissa & Spooner \\
\hline Bhavesh & Lallu \\
\hline Bronwen & Pepperell \\
\hline John & Chalissery \\
\hline Karim & Mahawish \\
\hline Susan & DeCaigney \\
\hline Paula & Broughton \\
\hline Karen & Knight \\
\hline Veronica & Duque \\
\hline Harry & McNaughton \\
\hline Jeremy & Lanford \\
\hline Vivian & $\mathrm{Fu}$ \\
\hline Lai-Kin & Wong \\
\hline
\end{tabular}




\section{AFFINITY Assessment of fluoxetine in stroke recovery}

\section{ASSESSMENT OF FLUOXETINE IN STROKE RECOVERY (AFFINITY) TRIAL}

Formal title: An Australasian, investigator-driven, NHMRC funded, multi-centre, prospective, randomised, parallel group, double-blind, placebo-controlled trial to establish the effect(s) of routine administration of fluoxetine ( $20 \mathrm{mg}$ once daily) in patients with recent stroke

Short title: A multicentre randomised controlled trial to establish the efficacy of routine administration of fluoxetine in patients with a recent stroke.

\section{Trial Protocol}

Version 5, $18^{\text {th }}$ November 2015

\section{Australian Government}

National Health and Medical Research Council

Government of Western Australia Department of Health
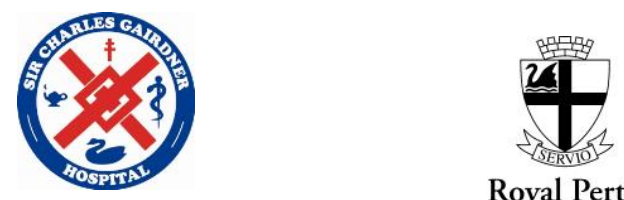

Royal Perth

Hospital

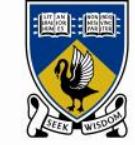

THE UNIVERSITY OF WESTERN AUSTRALIA
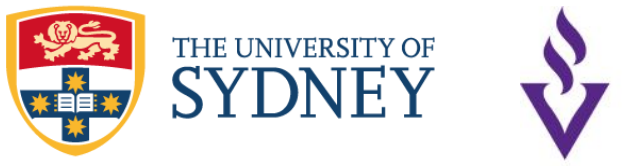
for Global Health 
Trial Co-Principal Investigators:

Associate Professor Maree Hackett, The George Institute for Global Health \& The University of Sydney

Professor Graeme J. Hankey, The University of Western Australia and Sir Charles Gairdner Hospital

Executive Steering Committee:

Professor Osvaldo Almeida, The University of Western Australia

Professor Craig S. Anderson, The George Institute for Global Health

Associate Professor Laurent Billot, The George Institute for Global Health

Professor Martin S. Dennis, The University of Edinburgh

Associate Professor Christopher Etherton-Beer, The University of Western Australia

Professor Leon Flicker, Royal Perth Hospital

Associate Professor Andrew Ford, The University of Western Australia

Professor Stephen Jan, The George Institute for Global Health

Professor Erik Lundström, Karolinska Institutet, Stockholm.

Professor Gillian Mead, The University of Edinburgh

Data Monitoring Committee:

Professor Robert Herbert, Neuroscience Research Australia

Professor Gregory Carter, University of Newcastle \& Calvary Mater Newcastle Hospital

Professor Geoffrey Donnan, The Florey Institute of Neurosciences \& Mental Health

Unblinded Statisticians:

Associate Professor Qilong Yi, Canadian Blood Services and University of Toronto

Dr Qiang Li, The George Institute for Global Health, Sydney

Administering Institutions:

- (Main point of contact for all trial inquiries)

The University of Western Australia \& Sir Charles Gairdner Hospital

AFFINITY Trial Coordinating Centre

Stroke Research Group

Harry Perkins Institute of Medical Research

$2^{\text {nd }}$ Floor, QQ Block

Sir Charles Gairdner Hospital

6 Verdun Street

Nedlands

Perth, Western Australia 6009

Ph: +61 8-6151 1061 Fax: +61 8-6151 1028

- The University of Western Australia

35 Stirling Highway, Crawley, Western Australia 6009

- The George Institute for Global Health

Street address:

Postal Address:

Level 10 King George V Building

PO Box M201

Royal Prince Alfred Hospital

Missenden Rd

83-117 Missenden Rd

Camperdown NSW 2050

Camperdown NSW 2050

Ph: +61 2-99934500 Fax: +61 2-99934502 


\section{Sponsors:}

Royal Perth Hospital and Sir Charles Gairdner Hospital

Funding:

National Health and Medical Research Council, Australia

Registration numbers:

Australian New Zealand Clinical Trial Registry number: ACTRN12611000774921 


\section{INVESTIGATOR AGREEMENT}

I have read this protocol and agree that it contains all the necessary details for carrying out the trial. I will conduct the trial as outlined herein and will complete the trial within the time designated.

I will provide copies of the protocol and all pertinent information to all individuals responsible to me who assist in the conduct of the trial. I will discuss this material with them to ensure that they are fully informed regarding the trial intervention and the conduct of the trial.

Name of Investigator at Participating site (Printed)

Name of Institution (Printed)

Site Investigator's Signature

Date (Day / Month / Year)

Trial Co-Principal Investigator

Associate Professor Maree Hackett

Neurological \& Mental Health Division

The George Institute for Global Health

Level 10, KGV Building

Missenden Road

Camperdown NSW 2050

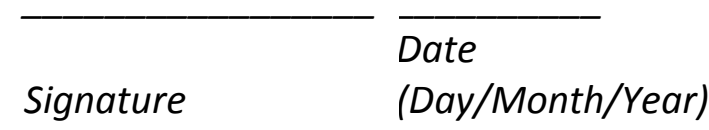

Trial Co-Principal Investigator

Professor Graeme Hankey

School of Medicine and Pharmacology,

The University of Western Australia

Room 222, Harry Perkins Institute of Medical

Research

Signature

Date

QQ Building, QEII Medical Centre,

(Day/Month/Year)

6 Verdun Street,

Nedlands, Perth, Western Australia, 6009 


\section{CONTENTS}

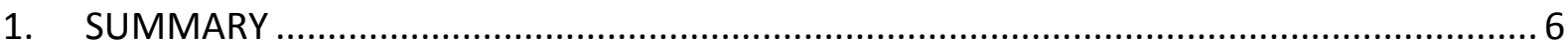

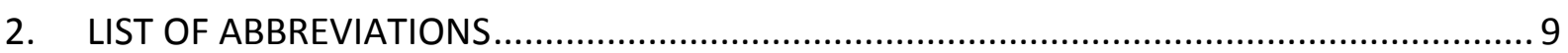

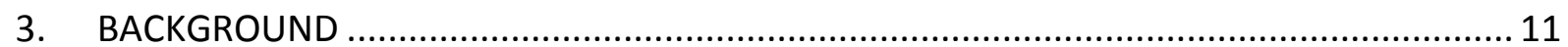

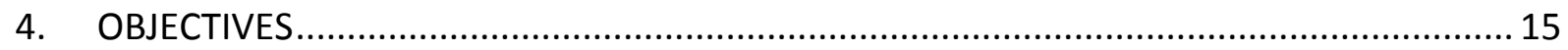

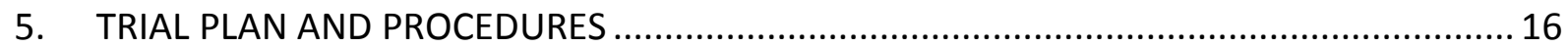

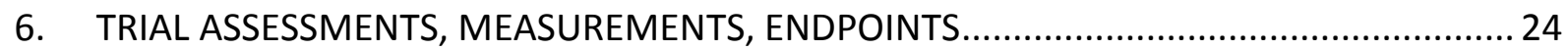

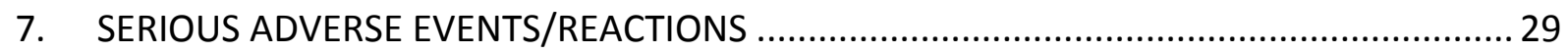

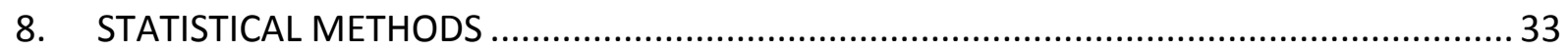

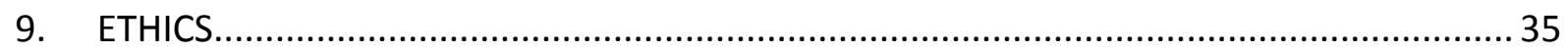

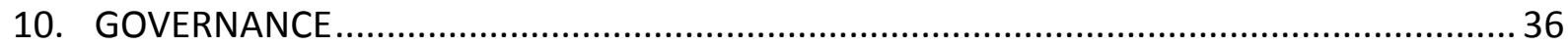

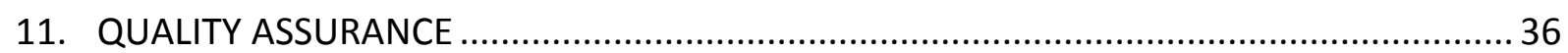

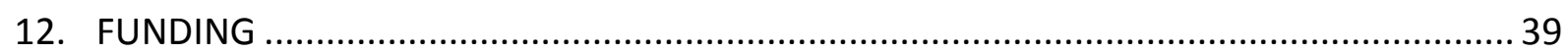

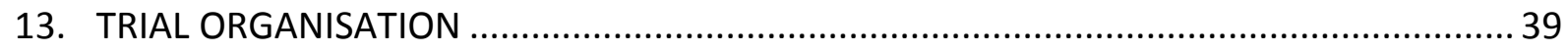

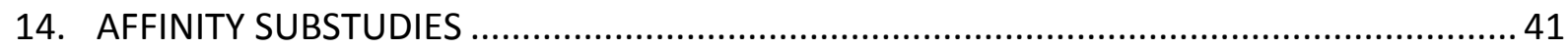

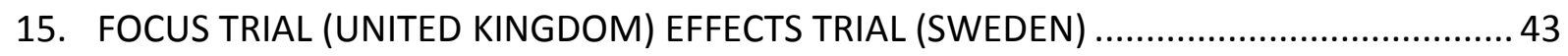

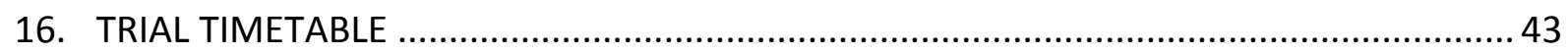

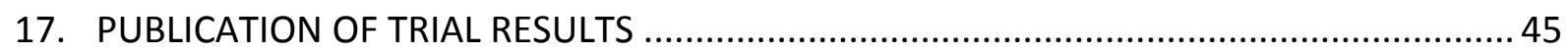

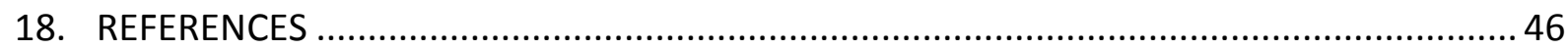

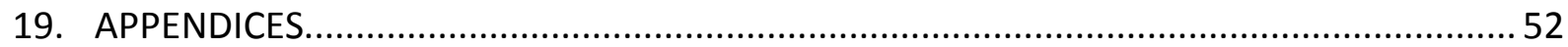

APPENDIX 1: NATIONAL INSTITUTE OF HEALTH STROKE SCORE ........................................ 53

APPENDIX 2: SIMPLIFIED MODIFIED RANKIN SCALE QUESTIONNAIRE..................................58

APPENDIX 3: OXFORDSHIRE COMMUNITY STROKE PROJECT CLASSIFICIATION OF CLINICAL

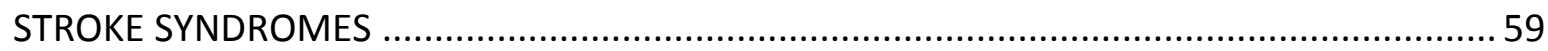

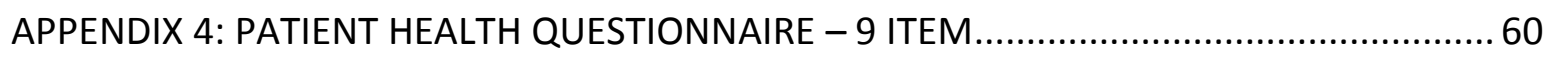

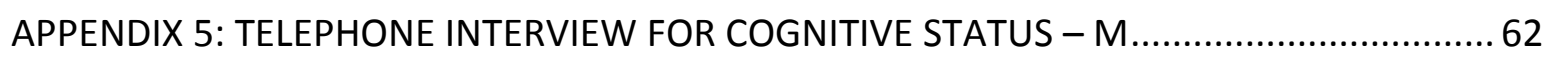

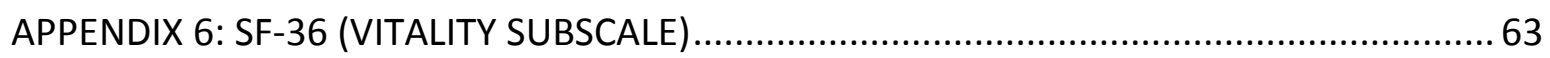

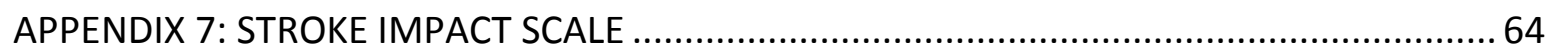

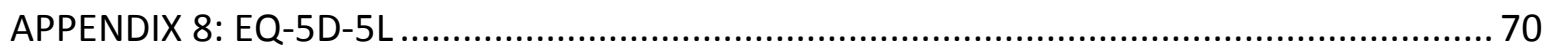

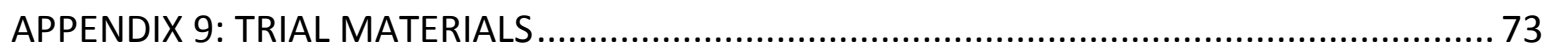

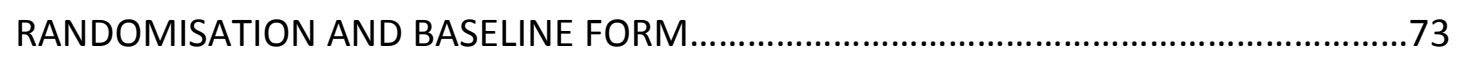

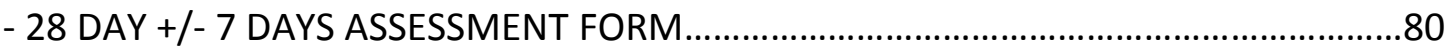

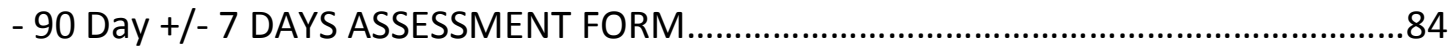

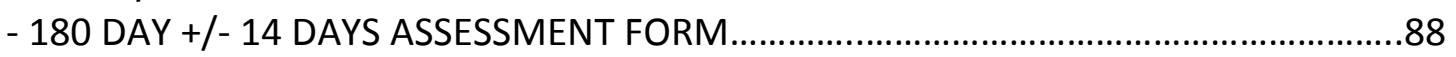

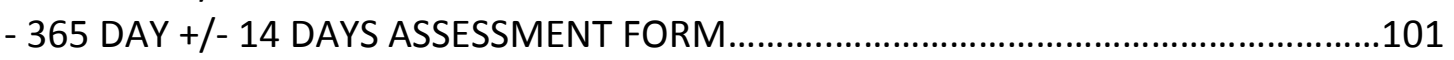




\section{SUMMARY}

\begin{tabular}{|c|c|}
\hline Title & $\begin{array}{l}\text { An Australasian, investigator-driven, NHMRC funded, multi-centre, } \\
\text { prospective, randomised, parallel group, double-blind, placebo-controlled } \\
\text { trial to establish the effect(s) of routine administration of fluoxetine ( } 20 \mathrm{mg} \\
\text { once daily) in patients with recent stroke }\end{array}$ \\
\hline Short title & Assessment of fluoxetine in stroke recovery (AFFINITY) trial \\
\hline Acronym & AFFINITY \\
\hline Clinical phase & $\begin{array}{l}\text { IIIb (i.e. fluoxetine is an established drug for depression, but not for stroke } \\
\text { recovery; hence, possible new indication) }\end{array}$ \\
\hline $\begin{array}{l}\text { Trial } \\
\text { Co-Principal } \\
\text { Investigators }\end{array}$ & $\begin{array}{l}\text { Associate Professor Maree Hackett, The George Institute for Global Health \& } \\
\text { The University of Sydney } \\
\text { Professor Graeme Hankey, The University of Western Australia \& Sir Charles } \\
\text { Gairdner Hospital }\end{array}$ \\
\hline $\begin{array}{l}\text { Primary } \\
\text { Research } \\
\text { Question }\end{array}$ & $\begin{array}{l}\text { Does treatment with fluoxetine, } 20 \text { mg once daily, started 2-15 days after } \\
\text { stroke onset and continued for } 180 \text { days, improve functional outcome at } 180 \\
\text { days after randomisation? }\end{array}$ \\
\hline Trial design & Parallel group, randomised, placebo-controlled clinical trial. \\
\hline Setting & Australian and New Zealand hospital stroke units and rehabilitation centres \\
\hline $\begin{array}{l}\text { Eligibility } \\
\text { criteria }\end{array}$ & 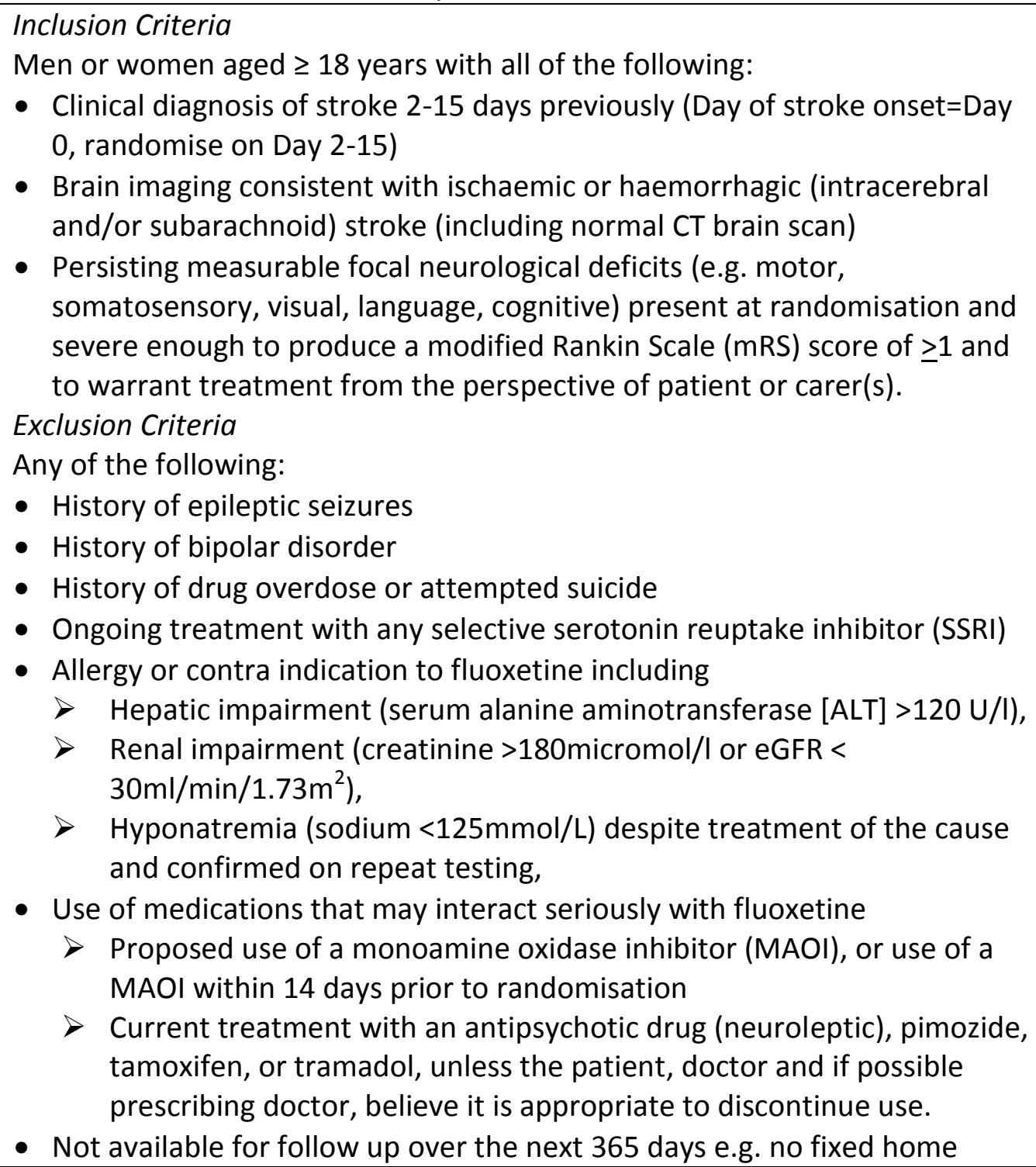 \\
\hline
\end{tabular}




\begin{tabular}{|c|c|}
\hline & $\begin{array}{l}\text { - Lifdre-threatening illness (e.g. advanced cancer) that is likely to reduce } 365 \\
\text { day survival } \\
\text { - Pregnant, breast-feeding or of child-bearing potential and not using } \\
\text { contraception } \\
\text { - Enrolled in another interventional clinical research trial involving an } \\
\text { investigational product (medicine) or device }\end{array}$ \\
\hline Randomisation & $\begin{array}{l}\text { Randomisation is by means of a password protected, computerised central } \\
\text { randomisation service available } 24 \text { hours-a-day, using a minimisation } \\
\text { algorithm to achieve balance between the two treatment groups for the } \\
\text { following four prognostic factors: } \\
\text { - Time from stroke onset ( } 2-8 \text { vs } 9-15 \text { days) } \\
\text { - Presence of a motor deficit } \\
\text { - Presence of aphasia } \\
\text { - Predicted probability of survival free of dependency at } 6 \text { months ( } 0-15 \% \\
\text { vs } 16-100 \%) \text {. }\end{array}$ \\
\hline Interventions & $\begin{array}{l}\text { Participants are randomly assigned to } 180 \text { days of treatment with either: } \\
\text { - Fluoxetine, } 20 \mathrm{mg} \text { capsules, to be taken once daily, or } \\
\text { - Placebo capsules that match the fluoxetine capsules, once daily. } \\
\text { For participants unable to swallow, the contents of an opened capsule can be } \\
\text { given via enteral tube. }\end{array}$ \\
\hline $\begin{array}{l}\text { Outcome } \\
\text { measures }\end{array}$ & $\begin{array}{l}\text { Primary outcome } \\
\text { - Functional outcome as measured by the mRS using the simplified } \\
\text { modified Rankin Scale questionnaire (smRSq) at } 180 \text { days after } \\
\text { Secondary outcomes at } 180 \text { and } 365 \text { days after randomisation } \\
\text { - Survival, } \\
\text { - Mood (Patient Health Questionnaire-9 item [PHQ-9] [46]), } \\
\text { - Cognitive function (Telephone Interview of Cognitive Status [TICSm] [47]), } \\
\text { - Communication (Stroke Impact Scale [SIS] [48]); } \\
\text { - Motor function (SIS [48]); } \\
\text { - Overall health status (SIS [48]); } \\
\text { - Health-Related Quality of Life (HRQoL) (EuroQoL [EQ-5D-5L] [49]); and } \\
\text { - Functional ability (smRSq [44, } 45] \text { ]) at the } 365 \text { day assessment. } \\
\text { - New diagnosis of depression requiring treatment with antidepressants; } \\
\text { - Fatigue (vitality domain of the Short Form } 36 \text { item, SF-36 [50, 51]); } \\
\text { Serious adverse events at any time during follow-up and which are also } \\
\text { recorded as other secondary outcomes } \\
\text { - New Stroke, ischaemic or haemorrhagic [not the qualifying event leading } \\
\text { - to enrolment] } \\
\text { - Acute coronary syndrome [Myocardial infarction confirmed by ECG and/ } \\
\text { - Up raised serum Troponin] } \\
\text { - Other mastrointestinal bleed requiring blood transfusion and/or } \\
\text { transfusion or procedural intervention }\end{array}$ \\
\hline
\end{tabular}




\begin{tabular}{|c|c|}
\hline & $\begin{array}{l}\text { - } \text { Fall } \\
\text { - New fracture [confirmed on X ray] } \\
\text { - } \quad \text { Epileptic seizure [focal or generalised] } \\
\text { - Symptomatic hypoglycaemia [blood sugar }<3 \mathrm{mmol} / \mathrm{I}] \\
\text { - Symptomatic hyperglycaemia [blood sugar }>22 \mathrm{mmol} / \mathrm{I}] \\
\text { - New hyponatraemia }[\mathrm{Na}<125 \mathrm{mmol} / \mathrm{I}] \\
\text { - } \text { Attempted suicide/self-harm } \\
\text { - Death } \\
\text { Cost of health care over the first year } \\
\text { Cost-effectiveness }\end{array}$ \\
\hline Follow up & $\begin{array}{l}\text { At } 28,90,180 \text { and } 365 \text { days after randomisation. } \\
\text { Participants are assessed by the site investigator at } 28 \text { and } 90 \text { days after } \\
\text { randomisation, in the hospital ward, outpatient clinic or via telephone. } \\
\text { Participants are followed-up at } 180 \text { and } 365 \text { days after randomisation by the } \\
\text { trial coordinating centre, by telephone or postal questionnaire. }\end{array}$ \\
\hline $\begin{array}{l}\text { Sample size } \\
\text { estimate }\end{array}$ & $\begin{array}{l}90 \% \text { power to detect an absolute increase in the proportion of participants } \\
\text { with an mRS of } 0-2 \text { at } 6 \text { months from } 50 \% \text { to } 57.5 \%\end{array}$ \\
\hline $\begin{array}{l}\text { Number of } \\
\text { participants }\end{array}$ & 1,600 (800 in each group) \\
\hline $\begin{array}{l}\text { Statistical } \\
\text { methods }\end{array}$ & $\begin{array}{l}\text { An ordinal logistic regression analysis of the mRS adjusted for baseline } \\
\text { variables included in minimisation algorithm }\end{array}$ \\
\hline Trial duration & $2013-2018$ \\
\hline
\end{tabular}

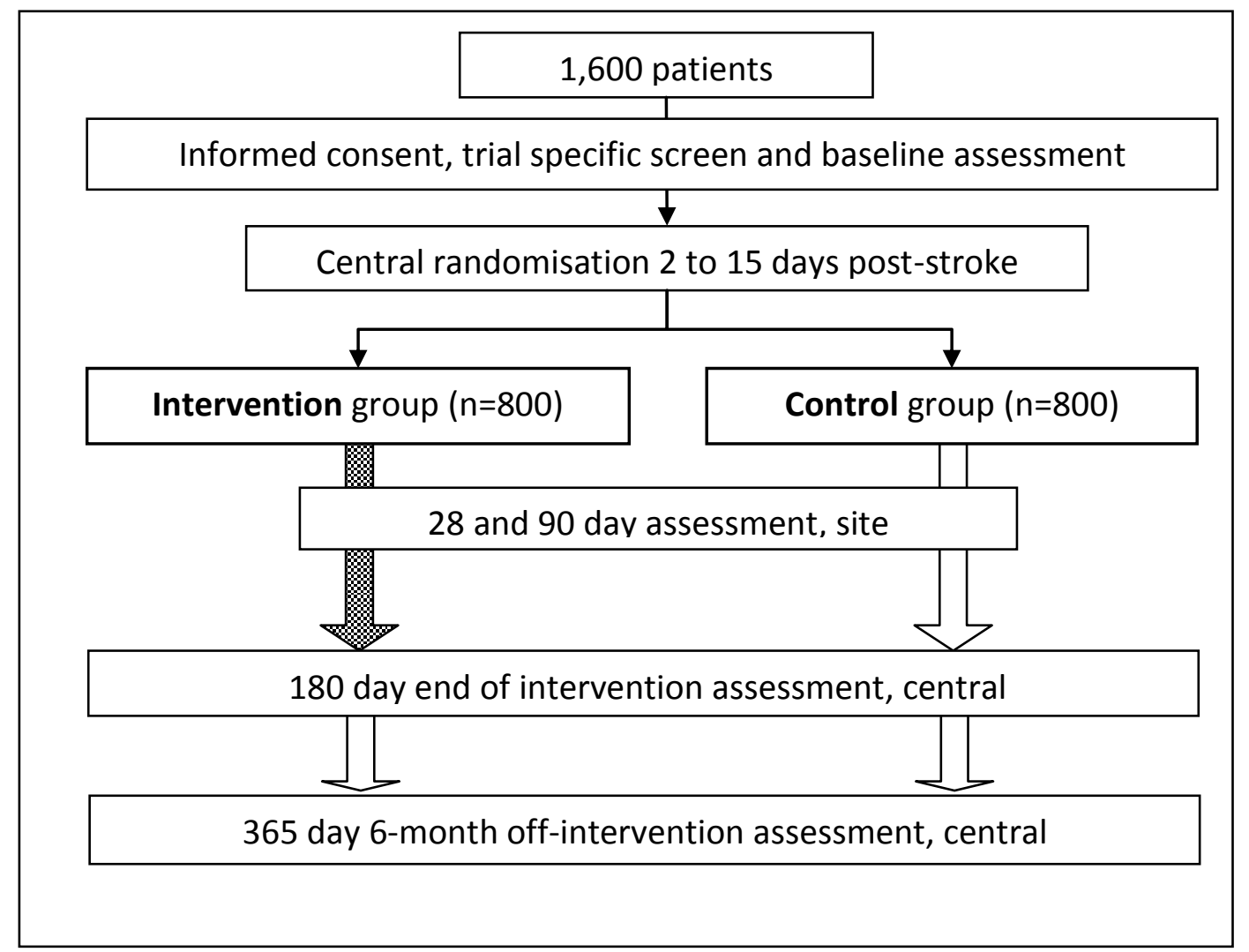

Figure 1. Flow summary of trial participants and assessments 


\section{LIST OF ABBREVIATIONS}

ADL/IADL activities of daily living/instrumental activities of daily living

ALT

Alanine Aminotransferase

BDNF brain derived neurotrophic factor

CAMP cyclic adenosine monophosphate

cGMP current Good Manufacturing Processes

$\mathrm{Cl} \quad$ confidence interval

CLOTS Clots in Legs Or sTockings after Stroke

CNS Central nervous system

CRF Case Report Form

CT Computerised Tomography

CV Curriculum Vitae

DALYs Disability adjusted life years

DMC Data monitoring committee

DSM-IV Diagnostic and Statistical manual of mental disorders-fourth edition

EFFECTS Swedish multicentre randomised placebo-controlled trial to establish the efficacy of fluoxetine in patients with a recent stroke

eGFR Glomerular Filtration Rate

EQ-5D-5L EuroQol 5 dimensions 5 levels

FLAME FLuoxetine for motor recovery after Acute ischaeMic strokE

FMMS Fugl-Meyer Motor Scale Score

fMRI functional Magnetic resonance Imaging

FOCUS Fluoxetine Or Control Under Supervision

GCP Good clinical practice

GCS Glasgow Coma Scale

GP General Practitioner

GSK GlaxoSmithKline

HR hazard ratio

HREC Health review ethics committee

HRQoL Health Related Quality of Life

$\mathrm{ICH} \quad$ International conference on harmonisation

IMP Investigational medicinal product

IQR Interquartile Range

ISF Investigator site file

MAOI Monoamine oxidase inhibitors

mRS modified Rankin Scale

NHMRC National Health and Medical Research Council

NIHSS National Institutes of Health Stroke Scale

OCSP Oxfordshire Community Stroke Project

OD once daily

OR odds ratio

PCREB Phosphorylated cAMP response element binding

PHQ-9 Patient Health Questionnaire-9 item

POISE Psychosocial Outcome In Stroke

PISC Participant Information and Consent Form

PPP Pharmaceutical packaging professionals

QALY Quality adjusted life year 


$\begin{array}{ll}\text { RCTs } & \text { Randomised Controlled Trials } \\ \text { RR } & \text { risk ratio } \\ \text { SAE } & \text { Serious Adverse Event } \\ \text { SAR } & \text { Serious Adverse Reaction } \\ \text { SCAST } & \text { Scandinavian Candesartan Acute Stroke Trial } \\ \text { SD } & \text { Standard deviation } \\ \text { SF-36 } & \text { Vitality Subscale of the Short Form-36 } \\ \text { SIADH } & \text { Syndrome of inappropriate anti-diuretic hormone } \\ \text { SIS } & \text { Stroke Impact Scale } \\ \text { SmRSq } & \text { Simplified modified Rankin Scale questionnaire } \\ \text { SSRI } & \text { selective serotonin reuptake inhibitor } \\ \text { SUSAR } & \text { Suspected Unexpected Serious Adverse Reaction } \\ \text { TGA } & \text { Therapeutic Goods Administration } \\ \text { TICSm } & \text { Telephone Interview for Cognitive Status- m } \\ \text { TOAST } & \text { Trial of org 10172 in acute stroke treatment } \\ \text { UK } & \text { United Kingdom } \\ \text { USA } & \text { United States of America } \\ \text { VITATOPS } & \text { VITAmins TO Prevent Stroke }\end{array}$




\section{BACKGROUND}

Long-term disability after stroke is common, and is the third most common cause of disability adjusted life years (DALYs)

About 60,000 Australians experience a stroke each year [1,2]. Thrombolysis, endovascular thrombectomy, antiplatelet therapy and stroke unit care facilitate the one-year survival of about 48,000 ( $80 \%$ ) but $15,000-24,000$ (30-50\% of survivors) remain disabled [3-5]. In 2010, stroke was the third leading cause of disability-adjusted life years (DALYs) worldwide among 291 diseases and injuries [6].

\section{Treatments that improve recovery and reduce disability after stroke are lacking}

One reason for the substantial disability after stroke is a shortage of effective, safe, affordable and accessible interventions that facilitate functional recovery [7]. Numerous clinical trials have failed to translate promising experimental data into clinically effective therapies [8]. However, hope has been re-ignited by the publication of the results of the fluoxetine for motor recovery after acute ischaemic stroke (FLAME) trial in 2011 (see page 12) [9].

\section{Fluoxetine is a promising neuroprotective and neuroregenerative agent}

Fluoxetine is a selective serotonin reuptake inhibitor (SSRI) that is commonly used to treat depression. It is also a promising neuroprotective and neuroregenerative / neurorestorative agent.

\section{Animal studies indicate that SSRIs may be of benefit to stroke survivors}

Animal models have produced results consistent with the hypothesis that fluoxetine, and possibly other SSRIs, might improve the clinical outcome of stroke survivors in a number of ways. First, brain stem and spinal cord $\alpha$-motor neurons receive dense serotonergic inputs, and serotonergic fibres innervate secondary motor structures such as the basal ganglia $[10,11]$. Second, SSRIs have a neurotrophic effect and stimulate neurogenesis in the adult brain [12-17]. They stimulate the secretion of growth factors, and other proteins associated with increased plasticity such as brain-derived neurotrophic factor (BDNF) [8,9] and phosphorylated cAMP response element binding (PCREB) protein [12-16], cell proliferation and the number of newborn neurons derived from the adult subgranular zone of the dentate gyrus in the hippocampus increases after prolonged treatment with fluoxetine [17]. Third, fluoxetine may have a neuroprotective role associated with its anti-inflammatory effect [18], thereby leading to a reduction of infarct size and enhancement of the expression of proteins (e.g. hypoxia-inducible factor 1 alpha) that facilitate recovery from ischaemic injury [19].

\section{Human studies indicate that fluoxetine use may improve stroke recovery}

Functional magnetic resonance imaging (fMRI) studies have found that fluoxetine can modulate motor activity in healthy subjects [20]. A subsequent double-blind placebocontrolled crossover trial reported that a single dose of fluoxetine improved motor performance and increased $\mathrm{fMRI}$ activation in 8 patients who had a lacunar stroke resulting in a pure motor hemiparesis [21]. These findings were replicated in a trial of 10 stroke patients [22]; participants who took a single dose of fluoxetine $20 \mathrm{mg}$ showed increased muscle activation in the paretic arm. Among 52 post-stroke hemiplegic participants who were receiving physiotherapy and were randomly assigned to fluoxetine $20 \mathrm{mg}$ daily, maprotiline $150 \mathrm{mg}$ daily or placebo for 3 months, those assigned fluoxetine showed the greatest functional improvement as judged by a graded neurological scale and a measure of activities of daily living (Barthel Index) [23,24]. In another randomised trial, daily 
administration of $10 \mathrm{mg}$ of the SSRI citalopram to stroke patients for 4 months was associated with a reduction in neurological impairment National Institute of Health Stroke Scale (NIHSS) score (appendix 1) of 2.3 versus 3.5 control, $p=0.03$ ) at trial completion [25]. In another randomised trial of fluoxetine $(n=32)$, nortriptyline $(n=22)$ and placebo $(n=29)$, three months of treatment with either nortriptyline or fluoxetine produced greater improvements in the modified Rankin Scale (mRS) measured at 1 year (i.e., 9 months after the start of treatment) compared with placebo, after adjusting for age depression, stroke severity, and rehabilitation intensity [26]. The significant benefits of treatment, as measured by the mRS compared with placebo, continued for at least 1 year.

The fluoxetine for motor recovery after acute ischemic stroke (FLAME) trial in humans.

Among 118 patients with ischaemic stroke 5-10 days earlier, hemiparesis and a Fugl-Meyer Motor Scale Score $(F M M S)<55$, random allocation to double-blind treatment with fluoxetine $20 \mathrm{mg}$ daily $(n=57)$ for 3 months, compared with placebo $(n=56)$, improved motor function (adjusted mean FMMS 34.0 vs 24.3 points; $p=0.003$ ), functional independence mRS score of $0-2: 26.3 \%$ vs $8.9 \%$; odds ratio [OR]: $3.8,95 \%$ confidence interval [ $\mathrm{Cl}]: 1.2$ to 10.7 ), and maintained participants free of depression (96\% vs $71 \% ; p=0.002$ ) [9]. Adverse effects included transient nausea, diarrhoea, and abdominal pain (14 [25\%] fluoxetine vs 6 [11\%] placebo). Despite its encouraging results, the FLAME trial has raised several questions [27]:

\section{Are the results of the FLAME trial valid?}

Although systematic error was minimised by double-blinding and randomisation, random error might have occurred because of the small sample size $(n=118)$ and limited statistical power.

Other studies in humans: a systematic review of all randomised controlled trials of fluoxetine In order to minimise random error and explore external validity (generalisability), we completed a Cochrane systematic review and meta-analysis of 52 randomised controlled trials (RCTs) of SSRIs on recovery after stroke in a total of 4,059 patients [26]. Overall, use of SSRIs was associated with less dependency (risk ratio [RR] $0.81,95 \% \mathrm{Cl}: 0.68$ to 0.97 ) (two trials, $n=223$ ) at the end of treatment compared with control (placebo or usual care) [28] (see Figure 2),

1.1 Dependent on modified Rankin score (mRS 3 to 5 )

\begin{tabular}{|c|c|c|c|c|c|c|c|c|}
\hline \multirow{2}{*}{ Study or Subgroup } & \multicolumn{2}{|c|}{ SSRI } & \multicolumn{2}{|c|}{ Control } & Weight & Risk Ratio & \multicolumn{2}{|c|}{$\begin{array}{c}\text { Risk Ratio } \\
\text { M-H, Random, } 95 \% \mathrm{Cl}\end{array}$} \\
\hline & & & & & & & & \\
\hline $\begin{array}{l}\text { Chollet } 2011 \\
\text { Subtotal }(95 \% \mathrm{Cl})\end{array}$ & 42 & $\begin{array}{l}57 \\
57\end{array}$ & 50 & $\begin{array}{l}55 \\
55\end{array}$ & $\begin{array}{l}100.0 \% \\
100.0 \%\end{array}$ & $\begin{array}{l}0.81[0.68,0.97] \\
0.81[0.68,0.97]\end{array}$ & & \\
\hline $\begin{array}{l}\text { Total events } \\
\text { Heterogeneity: Not a } \\
\text { Test for overall effect }\end{array}$ & $\begin{array}{r}42 \\
\text { plicable } \\
Z=2.34\end{array}$ & $P=0.0$ & 50 & & & & & \\
\hline 1.1.2 Sertraline & & & & & & & & \\
\hline $\begin{array}{l}\text { Almeida } 2006 \\
\text { Subtotal }(95 \% \mathrm{Cl})\end{array}$ & 0 & $\begin{array}{l}55 \\
55\end{array}$ & 0 & $\begin{array}{l}56 \\
56\end{array}$ & & $\begin{array}{l}\text { Not estimable } \\
\text { Not estimable }\end{array}$ & & \\
\hline $\begin{array}{l}\text { Total events } \\
\text { Heterogeneity: Not a } \\
\text { Test for overall effect }\end{array}$ & $\begin{array}{l}\quad 0 \\
\text { plicable } \\
\text { Not appli }\end{array}$ & cable & 0 & & & & & \\
\hline Total $(95 \% \mathrm{Cl})$ & & 112 & & 111 & $100.0 \%$ & $0.81[0.68,0.97]$ & $\bullet$ & 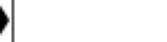 \\
\hline Total events & 42 & & 50 & & & & & \\
\hline $\begin{array}{l}\text { Heterogeneity: Not a } \\
\text { Test for overall effect } \\
\text { Test for subgroun }\end{array}$ & $\begin{array}{l}\text { plicable } \\
Z=2.34 \\
\text { erences: }\end{array}$ & $P=0.0$ & $\begin{array}{l}\text { 12) } \\
\text { alicable }\end{array}$ & & & & $\begin{array}{lc}0.01 & 0.1 \\
& \text { Favours SSRI }\end{array}$ & $\begin{array}{l}1 \\
1 \\
\text { Favours co }\end{array}$ \\
\hline
\end{tabular}

Figure 2. Risk ratio of dependence, defined by modified Rankin Scale score (mRS score 3-5) [28]. 
and less disability (standard mean difference $0.91,95 \% \mathrm{Cl}: 0.60$ to 1.22 ) ( 22 trials, $\mathrm{n}=1343$ ) at the end of treatment compared with control (placebo or usual care) (see Figure 3) [28].

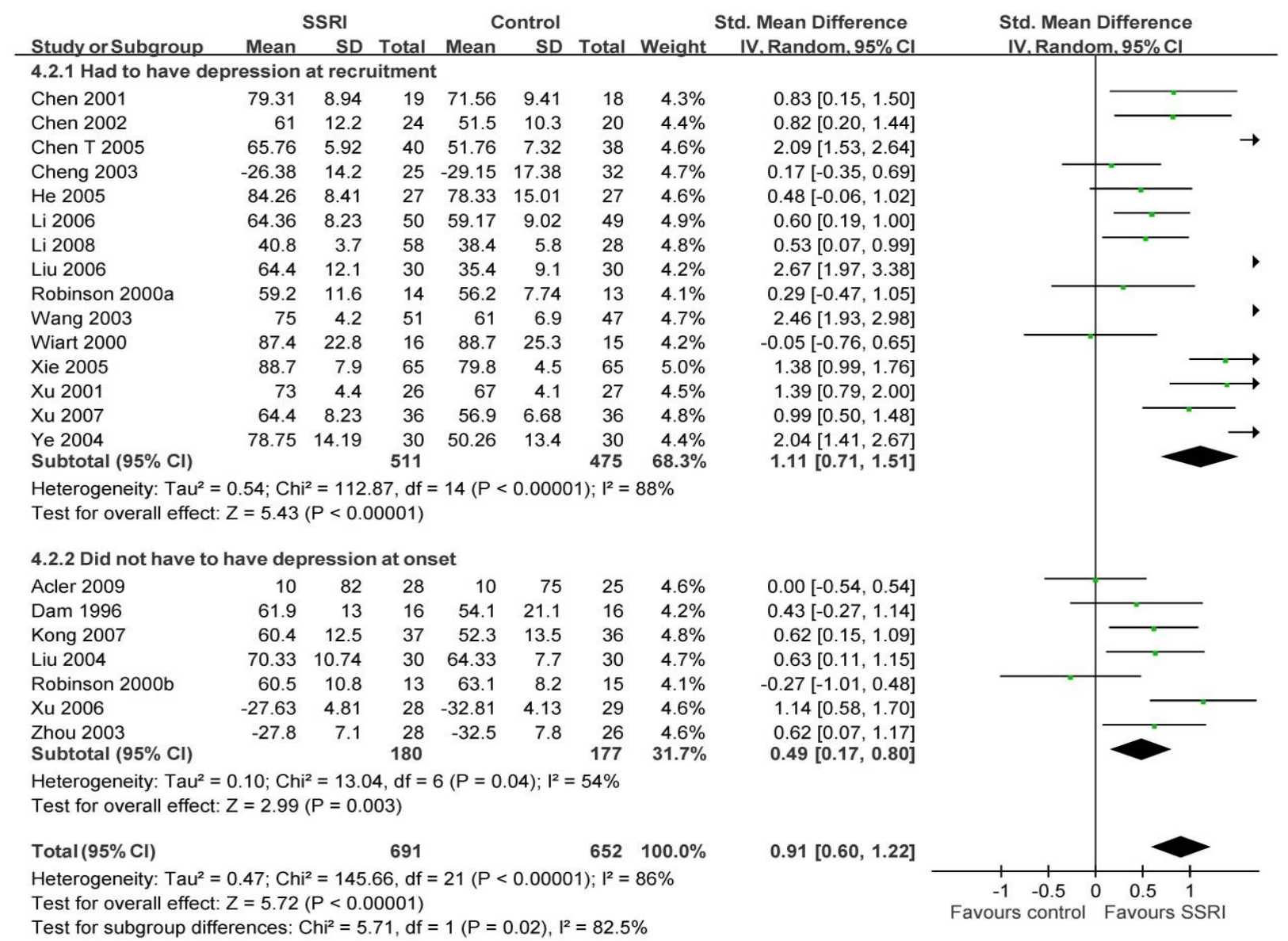

Figure 3. Standard mean difference in disability between SSRI and control groups at the end of treatment.

However, the validity of these results is compromised by substantial heterogeneity among the trials $\left(I^{2}=86 ; p<0.0001\right)$, methodological limitations (e.g. lack of blinding, incomplete outcome data), and clinical heterogeneity (e.g. many trials excluded aphasic and cognitively impaired patients). Further, adverse event data were inadequate to determine risks, only 7 trials ( $n=495)$ followed patients after treatment ended, and trials at low risk of bias reported smaller treatment effects.

Moreover, only 12 trials of fluoxetine were placebo-controlled in a total of only 682 patients, and an average treatment duration of only 7 weeks. Of these 12 trials, the primary outcome was disability (binary) in only one trial and functional recovery (continuous) in two trials; 5 trials assessed functional recovery as one of many secondary endpoints, and no trial followed-up patients beyond the treatment period.

A meta-analysis of the 6 placebo-controlled trials of fluoxetine which measured degree of functional recovery showed that in a total of 277 patients fluoxetine was associated with less disability compared with placebo (standardized mean difference: $0.35,95 \% \mathrm{Cl} 0.03$ to 0.61 ) [28].

These data are not sufficiently robust to be conclusive or to change clinical practice. However, they are sufficiently promising to warrant further evaluation of the effects of 
fluoxetine on recovery after stroke. One or more large RCTs, conducted according to the world's best standards, would reliably answer the question $[29,30]$.

2. Are the results of FLAME \& other trials generalisable to patients without severe hemiparesis?

The effect of fluoxetine in patients with mild to moderate hemiparesis, disabling non-motor neurological deficits and severe disability (NIHSS score $>20$ ) is uncertain.

3. Are the results of FLAME \& other trials generalisable to non-motor outcomes?

The effects of fluoxetine on cognition, communication, quality of life, and fatigue are uncertain.

4. Do any beneficial effects of fluoxetine on recovery persist after it is stopped?

The effects of fluoxetine on recovery after fluoxetine is stopped have not been investigated yet.

$\underline{5 . ~ I s ~ t h e ~ e f f e c t ~ o f ~ f l u o x e t i n e ~ o n ~ r e c o v e r y ~ d u e ~ t o ~ a n t i d e p r e s s a n t ~ o r ~ n e u r o r e g e n e r a t i v e ~}$ effects?

It is uncertain if there is an interaction between mood and recovery. The FLAME trial results remained significant after adjusting for depression [9], but in our systematic review the effect of SSRIs on recovery was greater in people with depression at randomisation [28]. Even if fluoxetine does improve recovery only because of its favourable effects on mood (e.g. less depression may facilitate participation in rehabilitation and increased physical and cognitive activity), that would still be an important finding that would change clinical practice.

6. Does the risk of serious adverse events offset or exceed any benefits of fluoxetine?

Our systematic review indicated that SSRIs, compared with placebo or usual care, were associated with a non-significant excess of seizures (RR 2.7; 95\% Cl 0.6-11.6) (7 trials, 444 participants), gastrointestinal adverse effects (RR 1.9; 0.9-3.8) (14 trials, 902 participants) and bleeding (RR 1.6; 0.2-13) (2 trials, 249 participants) [28]. Cohort studies, whilst prone to confounding and indication bias, have also reported that SSRI use is associated with increased risk of seizures, bleeding and hyponatraemia, particularly during the first 4 weeks of treatment [31-35].

\section{Summary of fluoxetine as a promising neuroprotective and neuroregenerative agent}

Our Cochrane review suggests that fluoxetine has promising effects on stroke recovery, perhaps via neuroregeneration [28]. Further, fluoxetine is widely known, available and affordable (Aus\$4.50 per month; no longer patented). However, the data from our review are not sufficiently compelling to prove that fluoxetine improves functional recovery after stroke, that any effects are independent of its effects on mood, and that any possible favourable effects are not offset by serious adverse effects, particularly among older stroke patients taking antithrombotic agents [28]. As in several areas of medicine, clinical practice in stroke recovery has been influenced by premature reports of beneficial effects from small trials that have not been replicated by more methodologically robust studies [36-39]. It is not only ethical but also essential that sound large clinical trials are undertaken to provide valid and more precise estimates of the efficacy and safety of fluoxetine for post-stroke recovery and function [27,29,40-42]. Other leading investigators in the United Kingdom (UK) (GE Mead and MS Dennis), Sweden (E Lundström and the late V Murray) and United States of America (USA) also see the need for large RCTs $[30,43]$. The AFFINITY trial, whilst unique in many aspects, has been designed to allow future pooling of data with the concurrently ongoing UK Fluoxetine Or Control Under Supervision (FOCUS) trial (see section 15) and the 
Swedish multicentre randomised placebo-controlled trial to establish the efficacy of fluoxetine in patients with a recent stroke (EFFECTS) trial (see section 15).

\section{OBJECTIVES}

\section{Hypothesis}

Administration of fluoxetine (20mg once daily; (od) for the first 180 days after stroke improves functional ability at the 180 day assessment, and the effect persists for 180 days after fluoxetine is stopped.

\section{Primary Objective}

To determine if treatment with fluoxetine, $20 \mathrm{mg}$ once daily, started 2-15 days after stroke onset and continued for 180 days, improves functional ability at the 180 day assessment as measured by the mRS using the simplified modified Rankin Scale questionnaire (smRSq) $[44,45]$ (appendix 2).

\section{Secondary Objectives}

Secondary outcomes at 180 and 365 days after randomisation

- Survival;

- Mood (Patient Health Questionnaire-9 item [PHQ-9] [46]) (appendix 4);

- Cognitive function (Telephone Interview of Cognitive Status [TICSm] [47]) (appendix 5);

- Communication (Stroke Impact Scale [SIS] [48]) (appendix 7);

- Motor function (SIS [48]);

- Overall health status (SIS [48]);

- Health-Related Quality of Life (HRQoL) (EQ-5D-5L [49]) (appendix 8); and

- Functional ability (smRSq $[44,45]$ ) at the 365 day assessment.

- New diagnosis of depression requiring treatment with antidepressants;

- Fatigue (vitality domain of the short form- 36 [SF-36] [50, 51]) (appendix 6).

Serious adverse events which occur at any time during follow-up and which are also recorded as secondary outcome measures include:

$>$ New Stroke, ischaemic or haemorrhagic [not the qualifying event leading to enrolment]

$>$ Acute coronary syndrome [myocardial infarction confirmed by ECG and/or raised serum Troponin]

D Upper gastrointestinal bleed [requiring blood transfusion and/or endoscopy

$>$ Other major bleed (i.e. not upper $\mathrm{Gl}$ or intracerebral) requiring blood transfusion or procedural intervention

$>$ Fall

$>$ New fracture [confirmed on $X$ ray]

$>$ Epileptic seizure [focal or generalised]

$>$ Symptomatic hypoglycaemia [blood sugar $<3 \mathrm{mmol} / \mathrm{I}]$

$>$ Symptomatic hyperglycaemia [blood sugar $>22 \mathrm{mmol} / \mathrm{I}$ ]

$>$ New hyponatremia $[\mathrm{Na}<125 \mathrm{mmol} / \mathrm{I}]$

$>$ Attempted suicide/self-harm

$>$ Death

Cost of health care over the first year

Cost-effectiveness. 


\section{TRIAL PLAN AND PROCEDURES}

\section{Trial design}

Parallel group, randomised, placebo-controlled clinical trial in which the participants, site investigators, and assessors will all remain unaware (i.e. blinded) of the intervention assignments throughout the trial (i.e. "double-blind" for centres where the site investigator and assessor are the same individual; "triple-blind" for centres where the site investigator and assessor are distinct individuals).

\section{Setting and recruitment}

Participants are being recruited in Australian and New Zealand hospital stroke units and rehabilitation centres by site investigators trained in relevant aspects of the trial by members of the trial coordinating centre. Screening, assessments and consent are conducted by the site investigator and data on randomised patients will be entered into a secure internet site. Recruitment began in January 2013 and is expected to cease at the end of 2017.

\section{Participants}

Inclusion criteria

Men or women aged $\geq 18$ years with all of the following:

- Clinical diagnosis of stroke 2-15 days previously (Day of stroke onset = Day 0, randomise on Day 2-15)

- Brain imaging consistent with ischaemic or haemorrhagic (intracerebral and/or subarachnoid) stroke (including normal computerised tomography (CT) brain scan)

- Persisting measurable focal neurological deficits (e.g. motor, somatosensory, visual, language, cognitive) present at randomisation and severe enough to produce a modified Rankin Scale (mRS) score of $\geq 1$ and to warrant treatment from the perspective of patient or carer(s).

\section{Exclusion criteria}

Any of the following:

- History of epileptic seizures

- History of bipolar disorder

- History of drug overdose or attempted suicide

- Ongoing treatment with any selective serotonin reuptake inhibitor (SSRI)

- Allergy or contra indication to fluoxetine including

$>$ Hepatic impairment (serum alanine aminotransferase $[A L T]>120 \mathrm{U} / \mathrm{I}$ ),

$>$ Renal impairment (creatinine > 180 micromol/l or estimated Glomerular Filtration Rate $\left[\right.$ eGFR] $<30 \mathrm{ml} / \mathrm{min} / 1.73 \mathrm{~m}^{2}$ ),

$>$ Hyponatremia (sodium $<125 \mathrm{mmol} / \mathrm{L}$ ) despite treatment of the cause and confirmed on repeat testing,

- Use of medications that may interact seriously with fluoxetine

$>$ Proposed use of a monoamine oxidase inhibitor (MAOI), or use of a MAOI within 14 days prior to randomisation

$>$ Current treatment with an antipsychotic drug (neuroleptic), pimozide, tamoxifen, or tramadol, unless the patient, doctor and if possible prescribing doctor, believe it is appropriate to discontinue use.

- Not available for follow-up over the next 365 days; e.g. no fixed home address

- Life-threatening illness (e.g. advanced cancer) that is likely to reduce 365 day survival

- Pregnant, breast-feeding or of child-bearing potential and not using contraception 
- Enrolled in another interventional clinical research trial involving an investigational product (medicine) or device.

\section{Co-enrolment}

Co-enrolment in another clinical trial of a medicinal or surgical intervention is not permitted because the other intervention could affect the primary outcome measure in AFFINITY and, because it is not assigned at random to the AFFINITY trial patients, it may confound the AFFINITY trial results. Co-enrolment in observational studies is acceptable.

\section{Consent}

It is the primary responsibility of the investigator at each participating site to obtain informed consent from participants to participate in the AFFINITY trial for 365 days using one of the approved methods outlined in section 9 of this protocol.

\section{Randomisation}

Consenting participants who meet the inclusion and exclusion criteria are randomised 2 to 15 days after stroke onset by means of a computerised central randomisation service (www.affinitytrial.org) that is available 24 hours a day.

\section{Minimisation}

At the time of randomisation, the following six prognostic variables are collected: age, living alone, independence in activities of daily living before the stroke, the verbal component of the Glasgow Coma Scale (GCS) (as derived from the NIHSS), arm power, and ability to walk [52]. The trial website has a computer program installed that automatically calculates the probability of survival free of dependency at the 180 day assessment, based on the prediction model developed and validated by Counsell et al [52]. It is anticipated that about half of randomised patients will have a predicted probability of $0-15 \%$, and the other half 16 to $100 \%$ of being independent at the 180 day assessment.

After entering the baseline demographic and clinical data of the participant randomisation occurs using a minimisation algorithm to achieve balance between the two treatment groups for the following four prognostic factors:

1. Delay between stroke onset and randomisation (2-8 vs 9-15 days). As spontaneous recovery may be quicker in the first week, we want to ensure that there is no substantial difference between treatment groups in the number of patients randomised in the first week, and also no difference between treatment groups in the number of patients randomised in the second week.

2. Presence of a motor deficit. To ensure the prevalence of patients with and without a motor deficit is equal in both treatment groups.

3. Presence of aphasia. To ensure the prevalence of patients with and without aphasia is equal in both treatment groups.

4. Probability of survival free of dependency at 6 months (0.15 vs $0.16-1.0)$ [52]. To ensure that at baseline, patients in each treatment group have equal probability of being alive and independent (mRS: $0-2$ ) at the 180 day assessment.

The purpose of the minimisation process is to ensure an equitable prevalence or balance of major prognostic factors for survival free of dependency between the two treatment arms.

At randomisation, participants are assigned to the group which minimises the difference across all four variables but only with a probability of 0.8 (rather than 1.0). This adds a random element to the treatment assignment which means that the likely treatment allocation cannot be guessed in advance. 
At randomisation, the system automatically generates a treatment allocation in a ratio of 1:1 active drug (fluoxetine) or placebo. Participants are allocated an AFFINITY number and a treatment number, that corresponds to a treatment pack located at the local hospital pharmacy. An email notification is sent to the trial coordinating centre and the site investigator with the randomisation details (AFFINITY number and treatment number). The site investigator is then responsible for ensuring the randomisation notification and medication prescription are delivered to the local hospital pharmacy so the participant may commence the allocated treatment as soon as possible.

\section{Intervention}

Participants are randomly assigned to six months treatment with either:

- Fluoxetine, $20 \mathrm{mg}$ capsules, to be taken once daily, or

- Placebo capsules that match the fluoxetine capsules (in appearance (same colour and shape), weight and texture), to be taken once daily.

For participants unable to swallow, the contents of an opened capsule can be given via enteral tube.

The fluoxetine capsules are a Generic brand of capsule supplied by Pharmaceutical Packaging Professionals (PPP) PTY LTD in South Australia.

The placebo capsules are manufactured and bottled at PPP.

The bottles of active drug [fluoxetine] and placebo are labelled by PPP in accordance with the randomisation schedule, according to regulatory requirements, and stored at PPP in accordance with standards of the South Australian Department of Human Services in a cGMP (current good manufacturing processes) dedicated warehouse at below 25 degrees celsius.

The trial coordinating centre is responsible for ordering the trial medication for the individual sites from PPP.

PPP distributes the AFFINITY trial medication usually in blocks of 10 treatment packs (5 active and 5 placebo) directly to the local pharmacy departments of the participating sites at the request of the trial coordinating centre. Each treatment pack consists of two bottles per participant (i.e. each bottle will contain a 90 day supply of 110 capsules of either fluoxetine or matching placebo). This includes an extra 20 capsules in each bottle to allow for delays in follow-up appointments or lost trial medication. Participating sites will instruct their hospital pharmacies to store the product under appropriate conditions (below 25 degrees celsius).

Site investigators will prescribe 180 days [ 6 months] supply of the trial medication by means of a local hospital prescription to be dispensed to the participant at baseline. The treatment packs are dispensed by the local hospital pharmacy. All treatment packs will be returned to the trial coordinating centre at the 180 day follow up where they will be counted and forwarded to PPP for destruction.

Participants are encouraged to minimise any unintentional non-adherence by using simple strategies, such as linking tablet-taking to the same time as other medication or having breakfast. It is the responsibility of each site investigator to ensure that accurate records of trial medication prescriptions are maintained. 


\section{Drug interactions}

\section{Metabolism of fluoxetine}

Following oral administration, fluoxetine is well absorbed from the Gl tract. Oral bioavailability is estimated to be at least $60-80 \%$. Peak plasma concentrations occur within 68 hours following a single oral dose. Food does not affect the systemic bioavailability of fluoxetine but it delays the absorption by $1-2$ hours (not clinically significant). $95 \%$ is bound to human serum proteins

Fluoxetine appears to be extensively metabolized, likely in the liver, to norfluoxetine and other metabolites via isoenzymes of the cytochrome P450 system, including 2D6, 3A4, 2C9, $3 \mathrm{~A} 5$, and $2 \mathrm{C} 19$.

Norfluoxetine, the principal active metabolite, is formed via $\mathrm{N}$-demethylation of fluoxetine.

Fluoxetine has a slow elimination half-life of 1 to 3 days after acute administration and 4 to 6 days after chronic administration. Its active metabolite, norfluoxetine has an elimination half-life of 4 to 16 days after acute and chronic administration. The long elimination halflives of fluoxetine and norfluoxetine assure that, even when dosing is stopped, active drug substance will persist in the body for weeks (primarily depending on individual patient characteristics, previous dosing regimen, and length of previous therapy at discontinuation).

Genetic polymorphisms that may reduce the metabolism of serotonin (and thereby increase blood and brain concentrations of serotonin)

A subset (about 7\%) of the population has reduced activity of the drug metabolizing enzyme cytochrome P450 2D6 (CYP2D6) - they are referred to as poor metabolizers. When a poor metabolizer takes several doses of fluoxetine the fluoxetine may accumulate to higher than usual concentrations in the blood, resulting in a greater exposure to fluoxetine than occurs in patients who have normal activity of enzyme. This could lead to adverse drug reactions of fluoxetine including anorexia, nervousness, tremor, tachycardia, and seizures.

Drugs that may reduce the metabolism of serotonin (and thereby increase blood and brain concentrations of serotonin)

Medications that inhibit the enzyme CYP2D6, and thereby increase blood and brain concentrations of fluoxetine, include:

- Antiarrhythmic drugs: Amiodarone, Quinidine (a medicine for heart arrhythmias.

- Antipsychotic drugs: Haloperidol, Pimozide. Some evidence suggests a possible pharmacodynamic and/or pharmacokinetic interaction between some SSRIs and some antipsychotics, including possible elevation of blood levels of haloperidol and clozapine. Concurrent use of fluoxetine with pimozide increases the risk of QTC prolongation.

Drugs that may interact with fluoxetine to increase blood and brain concentrations of serotonin

Fluoxetine, which increases the amount of serotonin that can act on receptors in the blood and brain, may interact with other medications that also increase blood and brain 
concentrations of serotonin [53]. These medications, which should be avoided with fluoxetine, include:

- Other SSRIs

- Tramadol and other synthetic opiates

- Triptans, such as sumatriptan (Imitrex ${ }^{\circledR}$, GlaxoSmithKline (GSK)

- Tryptophan,

- Lithium

- MAOIs, such as phenelzine (Nardil ${ }^{\circledR}$, Parke-Davis), tranylcypromine (Parnate ${ }^{\circledR}, \mathrm{GSK}$ ), and selegiline (Eldepryl ${ }^{\circledR}$, Somerset; and others).

1. The concurrent use of fluoxetine with MAOIs is contraindicated.

2. After stopping MAOIs, fluoxetine should not be started until at least 14 days have elapsed (after stopping the MOAI).

3. After stopping fluoxetine, MAOls should not be started until at least 5 weeks have elapsed (after stopping the fluoxetine), since fluoxetine is slowly eliminated from the body; and

- The herbal remedy St John's Wort (Hypericum perforatum) can potentially also increase the levels of serotonin.

Consequences of an increase in blood and brain concentrations of serotonin (see also "Overdose" below)

\section{Serotonin syndrome}

The potential consequence of co-administration of fluoxetine with serotonergic drugs (see above) is an increase in blood and brain concentrations of serotonin and a cluster of symptoms called the serotonin syndrome.

The symptoms and signs of serotonin syndrome include: restlessness, irritability, altered behaviour, confusion, shivering, fever/hyperthermia, diarrhoea, tremor, hyperreflexia, rigidity, clonus, myoclonus, autonomic instability with rapid fluctuations of vital signs, seizures, and mental status changes that include extreme agitation progressing to delirium and coma.

Effect of fluoxetine on the blood concentrations of other drugs Inhibition of CYP2D6.

Fluoxetine is also a potent inhibitor of the liver enzyme, CYP2D6.

Hence, fluoxetine can alter the blood concentrations of other medications that are metabolized by CYP2D6, such as:

- Tricyclic antidpressants: amitriptyline, imipramine, and desipramine

- Antiepileptic drugs: carbamazepine, phenytoin and

- Antipsychotic drugs: thioridazine.

- Codeine

These drugs should be started at low dose in patients taking fluoxetine, otherwise toxicity with these drugs may arise.

In contrast, fluoxetine inhibits the conversion of codeine to its active form morphine and thereby diminishes the analgesic effect of codeine.

Similarly, fluoxetine, by inhibiting CYP2D6, also inhibits the conversion of tamoxifen to its active metabolite, thus reducing the amount of active drug to protect against breast cancer recurrence. 


\section{Inhibition of CYP2C9 and CYP2C19}

Fluoxetine also inhibits the CYP2C9 and CYP2C19 enzymes and thereby may interfere with the elimination of many medications including warfarin (an anticoagulant) and phenytoin (an antiepileptic medication). The effects of these medications therefore require careful monitoring.

\section{Potential Effects of Co-administration of Drugs Highly Bound to Plasma Proteins}

Because fluoxetine is tightly bound to plasma proteins, the administration of fluoxetine to a participant taking another drug which is tightly bound to protein (e.g. warfarin) may cause a shift in plasma concentrations potentially resulting in an adverse effect. Conversely, adverse effects may result from displacement of protein bound fluoxetine by other tightly bound drugs.

\section{Participant adherence and compliance}

Participants are asked to keep a record of their use of the trial and other medication (e.g. concurrent/additional medications).

Participants are encouraged to adhere to (i.e. continue) the allocated treatment and are asked to record the dates and reasons of any temporary or permanent discontinuation.

Participants will also be encouraged to comply with (i.e. consistently take) the allocated trial medication. They are asked to keep their capsule bottles and any remaining capsules for return to the trial coordinating centre in Perth at the 180 day assessment so that the compliance rate (the ratio of taken doses to total possible doses) can be calculated.

\section{Premature discontinuation of trial medication}

Participants who choose to stop taking the allocated treatment are still followed up for study outcome measures as per the study protocol and included in the primary intention-to-treat analysis.

The reason(s) for stopping the trial treatment prematurely is/are recorded in the participants case report form (CRF).

If withdrawal results from a Serious Adverse Event (SAE), Serious Adverse Reactions (SAR) or Suspected Unexpected Serious Adverse Reaction (SUSAR) the event is reported as per protocol (Please see section 7 of this protocol for more information regarding SAEs, SARs and SUSARs).

Participants may also choose to withdraw completely from the trial, so that no further data are collected on the participant. If the participant is willing, we will record the reason for any such withdrawal on the "withdrawal of consent" form.

\section{Emergency Un-blinding}

Un-blinding is strongly discouraged.

In the event of an emergency, such as a suspected serotonin syndrome, discontinue the trial medication and treat the participant in the usual manner.

If an emergency occurs and un-blinding is required please contact your local hospital pharmacy who will contact the 24 hour un-blinding hotline on 1800175 982, in Australia and [+61 438356411 ] in New Zealand, this service is provided by PPP. Your local hospital pharmacy is required to provide the participants AFFINITY treatment number to break the code and receive the treatment allocation i.e. active (fluoxetine) or placebo.

The trial medication must be discontinued after emergency un-blinding. 


\section{Overdose}

Symptoms of overdose of fluoxetine include nausea, vomiting, and seizures. Cardiovascular dysfunction, ranging from asymptomatic arrhythmias to cardiac arrest, can occur, as may pulmonary dysfunction, and central nervous system (CNS) dysfunction, ranging from excitation to coma.

Cardiac and vital sign monitoring is recommended, along with general symptomatic and supportive measures.

No specific antidote is known. Activated charcoal, which may be used with sorbitol, may be as or more effective than emesis or lavage. Forced diuresis, dialysis, haemoperfusion, and exchange transfusion are unlikely to be of benefit because fluoxetine is highly proteinbound.

In managing over dosage, consider the possibility of multiple drug involvement. An extended time for close medical observation may be needed in participants who have taken excessive quantities of a tricyclic antidepressant if they are also taking, or have recently taken, fluoxetine.

Fatalities attributed to overdose of fluoxetine alone are extremely rare; the severity is usually mild and the course benign.

\section{Stopping the trial medication at the $\mathbf{1 8 0}$ day assessment (or earlier)}

Sudden cessation of an SSRI may lead to a withdrawal syndrome characterised by symptoms including anxiety, restlessness, insomnia, headache and tremor. However, of all the SSRIs, fluoxetine has the longest half-life (4-6 days) and therefore a withdrawal syndrome is very uncommon and tapering of the dose (especially from only $20 \mathrm{mg}$ od) is not regarded as necessary.

\section{Possible Side Effects of Fluoxetine \\ Adverse effects and suggested management (Adapted from reference [54])}

\begin{tabular}{|c|c|c|}
\hline Adverse effect & Comment & Management \\
\hline Dizziness & $\begin{array}{l}\text { Occurs in about one in } 10 \text { people who take second } \\
\text { generation antidepressants but is less common with } \\
\text { fluoxetine }\end{array}$ & $\begin{array}{l}\text { Check blood pressure standing and lying; symptoms } \\
\text { usually improve over time; Ensure adequate fluid } \\
\text { intake }\end{array}$ \\
\hline Sedation & Not common but can occur & $\begin{array}{l}\text { Sedation may be desirable; it may improve over time. } \\
\text { Change time of dosing and treatment }\end{array}$ \\
\hline Dry mouth & Probably dose related & $\begin{array}{l}\text { Tolerance may develop; suggest sugarless gum or } \\
\text { saliva substitutes }\end{array}$ \\
\hline $\begin{array}{l}\text { Sexual dysfunction } \\
\text { [Male and female] }\end{array}$ & Common but often not asked about & $\begin{array}{l}\text { Discuss participant's willingness to continue trial } \\
\text { medication in view of stroke diagnosis, possible } \\
\text { positive effects of the medication and } 6 \text { month } \\
\text { treatment regime. Short term use of sildenafil [males] } \\
\text { could also be considered if clinically appropriate. }\end{array}$ \\
\hline Insomnia & $\begin{array}{l}\text { Common problem but hard to distinguish from } \\
\text { insomnia caused by depression }\end{array}$ & $\begin{array}{l}\text { Change time of dosing (earlier or later may help), pay } \\
\text { attention to sleep hygiene. Monitor participant's } \\
\text { mood [Possible new diagnosis of depression.] }\end{array}$ \\
\hline Suicidal thoughts & $\begin{array}{l}\text { Antidepressants may paradoxically increase suicidal } \\
\text { thoughts in those aged under } 30\end{array}$ & $\begin{array}{l}\text { Protocol specific assessment of the participant: } \\
\text { includes the PHQ-9 assessment which asks about } \\
\text { suicidal thoughts. No evidence exists that asking } \\
\text { about suicide makes people more likely to harm } \\
\text { themselves. Urgent clinical management of the } \\
\text { participant with suicidal ideation is required. . }\end{array}$ \\
\hline Anxiety & Often occurs when starting SSRIs & $\begin{array}{l}\text { Monitor participant and reassure that symptoms } \\
\text { usually subside. }\end{array}$ \\
\hline Hyponatraemia & $\begin{array}{l}\text { Particularly a problem in the elderly and more } \\
\text { common with SSRIs }\end{array}$ & $\begin{array}{l}\text { Check sodium levels if the patient is symptomatic and } \\
\text { treat clinically. Stopping the trial medication should }\end{array}$ \\
\hline
\end{tabular}




\begin{tabular}{|c|c|c|}
\hline Adverse effect & Comment & Management \\
\hline & & not be necessary unless symptoms persist. \\
\hline $\begin{array}{l}\text { Serotonin } \\
\text { syndrome }\end{array}$ & $\begin{array}{l}\text { Characterised by changes in mental state (eg } \\
\text { confusion or agitation), autonomic instability (eg } \\
\text { high temperature, shivering, sweating, changes in } \\
\text { blood pressure), and neuromuscular hyperactivity } \\
\text { (eg clonus or hyper-reflexia). Seen particularly with } \\
\text { SSRIs and other drugs that effect serotonin }\end{array}$ & $\begin{array}{l}\text { Stop the trial medication Use supportive measures } \\
\text { such as hydration, management of hyperthermia, and } \\
\text { benzodiazepines. Consider cyproheptadine or } \\
\text { chlorpromazine in severe cases }\end{array}$ \\
\hline $\begin{array}{l}\text { Discontinuation } \\
\text { syndrome }\end{array}$ & $\begin{array}{l}\text { More common with SSRIs that have a short half-life } \\
\text { (eg paroxetine or venlafaxine) }\end{array}$ & $\begin{array}{l}\text { Advise the participant that fluoxetine has a long half- } \\
\text { life and, as such, reducing dosage prior to } \\
\text { discontinuation is not considered necessary. }\end{array}$ \\
\hline
\end{tabular}

SSRIs=selective serotonin reuptake inhibitors.

Return and disposal of unused medication at the end of the treatment phase (180 day follow up)

All unused medication is returned to the trial coordinating centre in Perth for medication accountability and from there returned to PPP for disposal.

Returns will be coordinated by the trial office in Perth by secure express post.

\section{Lost trial medication / Replacement stock}

The trial coordinating centre will liaise with Pharmaceutical Packaging Professionals [PPP], supplying them with the participants AFFINITY and treatment allocation numbers and the details of the participating site's pharmacy for shipment of the replacement trial medication. The trial coordinating centre will liaise with the site Principal Investigator or their delegated staff so that the replacement medication can be prescribed and dispensed to the participant. 


\title{
6. TRIAL ASSESSMENTS, MEASUREMENTS, ENDPOINTS
}

Figure 4: Flow diagram summarising the process of the AFFINITY trial

\section{Identify patient with stroke}

\author{
ת) \\ Check eligibility \\ Consent \\ ป \\ Collect baseline data
}

Randomise

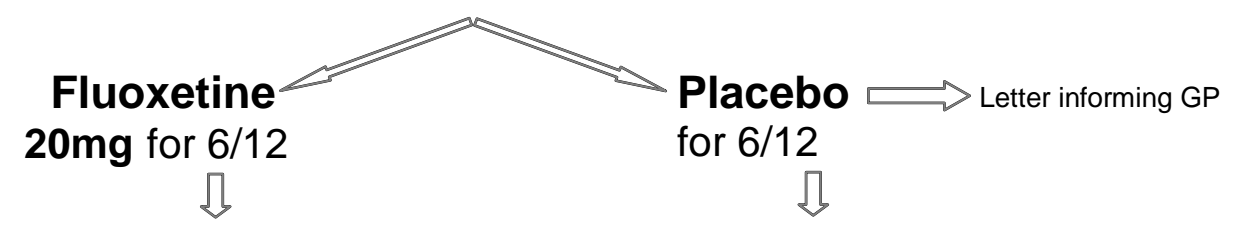

28 +/- 7 days: Site Telephone/Face to Face assessment on treatment

Survival, living arrangements, clarification of the final diagnosis and cause of qualifying stroke, current medications, medication compliance, serious adverse events, pregnancy (female participants of child bearing age), primary outcome (smRSq), current depression, mood (PHQ-9),

90 +/- 14 days: Site Telephone/Face to Face assessment on treatment

Survival, living arrangements, current medications, medication compliance, serious adverse events, pregnancy (female participants of child bearing age), primary outcome (smRSq), current depression, mood (PHQ-9)

180 +/- 14 days: Central Telephone/Postal assessment at end of treatment

Survival, living arrangements, current medications, medication compliance, serious adverse events, pregnancy (female participants of child bearing age), health care utilisation, primary outcome (smRSq), current depression, mood (PHQ-9), cognition (TICSm), Fatigue (vitality subscale of SF36), health-related quality of life (EQ-5D-5L), overall health status (SIS).

$365+/-14$ days: Central Telephone/Postal assessment at end of follow-up off treatment

Survival, living arrangements, current medications, serious adverse events, pregnancy (female participants of child bearing age), health care utilisation, primary outcome (smRSq), current depression, mood (PHQ-9), cognition (TICSm), Fatigue (vitality subscale of SF36), health-related quality of life (EQ-5D-5L), overall health status (SIS).

\section{Baseline assessment (2-15 days after stroke onset) (appendix 9)}

Prior to randomisation, the following are recorded: gender, date of birth, ethnicity, marital status, living arrangements, employment status, independence in activities of daily living before stroke, date of stroke, previous history of depression*, co morbidities, medications, classification of clinical stroke type (Oxfordshire Community Stroke Project, OCSP [55]) (appendix 3) ischaemic stroke subtype (Trial of Org 10172 in Acute Stroke Treatment [TOAST] criteria [56]), neurological impairments (NIHSS [57]) ; arm strength; ability to walk; functional ability (smRSq [44,45]), mood (PHQ-9 [46]), and the results of laboratory tests (serum creatinine, eGFR, sodium, ALT; pregnancy test if premenopausal woman; see below). 
*A history of "previous depression requiring previous treatment" is recorded at baseline. This should refer to a clinical diagnosis made by a GP, psychologist or hospital clinician prior to enrolment in AFFINITY. The responses to these questions should be completed before, and are independent of, the subsequent completion of the PHQ-9 and the associated score.

\section{Baseline Laboratory Tests}

A pregnancy test is performed on pre-menopausal women to confirm eligibility for the trial. Pregnant participants are excluded.

The following blood tests are performed at baseline and determine the eligibility of the participant for the trial.

Participants are excluded if:

- The ALT enzyme is 3 or more times above the upper limit of normal (>120U/I)

- The serum creatinine is $>180 \mathrm{micromol} / \mathrm{l}$

- $\quad$ eGFR is $<30 \mathrm{ml} / \mathrm{min} / 1.73 \mathrm{~m}^{2}$

- Blood sodium concentration is low ( $<125 \mathrm{mmol} / \mathrm{L})$

If the participant has an abnormal blood result at this baseline test please treat the cause, repeat the test and reassess before randomising.

Laboratory tests completed within 15 days post stroke onset are accepted as baseline measures.

\section{Randomisation}

The trial medication is dispensed and administered.

The contact sheet containing participant and GP contact details for central follow up is completed and filed in the participant AFFINITY folder.

The site will then be required to send a letter to the participant's GP, including details of the trial, management guidelines and the trial coordinating centre contact details.

Participants will also be asked to keep a record of any hospitalisations over the duration of the trial.

\section{8 and 90 day follow-up (appendix 9)}

At 28 days +/- 7 days and the 90 day +/- 14 days after randomisation, the participant will undergo a telephone or face to face interview with the randomising site staff to assess survival, living arrangements, clarification of the final diagnosis and cause of stroke (28 day follow-up only), current medications, medication compliance and tolerance, SAEs, pregnancy (female participants of child bearing age), primary outcome (mRS [44,45]), recent depression, and current mood (PHQ-9 [46]).

- Routine blood tests are not required

- Repeat blood tests (ALT, creatinine, eGFR and Blood sodium) may be necessary if the investigator deems it to be clinically indicated (ie the participant is clinically unwell and is displaying possible side effects of the trial medication [fluoxetine]).

If the blood sodium concentration during follow-up is low $(<125 \mathrm{mmol} / \mathrm{L}$, the cause of the hyponatraemia may be syndrome of inappropriate anti-diuretic hormone (SIADH) secretion.

If SIADH is confirmed, by low plasma sodium concentration and osmolality and an inappropriately high urinary osmolality, the recommended treatment is to treat or remove the cause (if it is known) and restrict fluid intake to $800 \mathrm{ml}-1$ Litre per day until the blood sodium concentration returns to normal. 
As Fluoxetine may cause SIADH, and the AFFINITY trial medication may contain fluoxetine (or placebo), it is recommended that the AFFINITY Trial medication should be withheld until the blood sodium concentration has returned to normal (e.g. within 3-7 days of fluid restriction).

Once the sodium concentration has returned to normal, the AFFINITY trial medication can be re-started if the patient is willing to take it again.

If the SIADH reoccurs, then the trial medication should be ceased permanently and this should be reported to the AFFINITY trial office.

\section{Completion of the 90 day follow up:}

When the 90 day follow up assessment has been completed and the participant contact details have been received by the trial coordinating centre, a letter of introduction will be sent to the participant from the trial coordinating centre, with the contact details of the trial coordinating centre.

The letter will also advise participants to notify their GP or specialist of their participation in the AFFINITY trial, and to raise any concerns they may have regarding their participation in the trial with their GP, specialist and/or the trial coordinating centre. Participants will also be advised to report any temporary or permanent discontinuations of the trial medication to the trial coordinating centre and will be advised regarding forthcoming central follow up at the 180 and 365 day assessments including the procedure for the return of trial medication on completion of their allocated treatment.

\section{0 and 365 day follow-ups (appendix 9)}

At 180 days +/- 14 days and at 365 days +/- 14 days the participant will be followed up centrally by means of a telephone interview with trial coordinating centre staff to assess survival, living arrangements, current medications, medication compliance (180 day followup only), SAEs, pregnancy (female participants of child bearing age), health care utilisation, primary outcome (mRS [44,45]), recent depression, current mood (PHQ-9 [46]), cognition (TICSm [47]), fatigue (vitality subscale of SF36 [50,51]), health-related quality of life (EQ-5D$5 \mathrm{~L}$ [49]) and overall health status (SIS [48]). The (SIS) can be delivered as a postal questionnaire.

Table 1. Trial assessment schedule

\begin{tabular}{|c|c|c|c|c|c|}
\hline & Baseline & 28 Day & 90 Day & 180 Day & 365 Day \\
\hline Informed Consent & $\mathrm{x}$ & & & & \\
\hline In/exclusion criteria & $x$ & & & & \\
\hline Demographics & $x$ & $x$ & $x$ & $x$ & $x$ \\
\hline Details of Stroke & $x$ & & & & \\
\hline Neurological Impairments (NIHSS [35]) & $x$ & & & & \\
\hline Functional Ability - smRSq [24, 25] & $x$ & $x$ & $x$ & $x$ & $x$ \\
\hline Classification of stroke - OCSP [30] & $x$ & $x$ & & & \\
\hline Ischaemic Stroke sub-type TOAST [34] & $x$ & $x$ & & & \\
\hline Clinical Depression & $x$ & $x$ & $x$ & $x$ & $x$ \\
\hline PHQ-9 [26] & $x$ & $x$ & $x$ & $x$ & $x$ \\
\hline Medical co-morbidities & $x$ & & & & \\
\hline Hospital admissions & & $x$ & $x$ & $x$ & $x$ \\
\hline Medications in use & $x$ & $x$ & $x$ & $x$ & $x$ \\
\hline Compliance monitoring & & $x$ & $x$ & $x$ & \\
\hline SUSARS/SAES & & $x$ & $x$ & $x$ & $x$ \\
\hline Cognition - TICSm [27] & & & & $x$ & $\mathrm{x}$ \\
\hline Overall Health Status - SIS [28] & & & & $x$ & $x$ \\
\hline
\end{tabular}


Health-related Quality of Life - EQ-5D-5L [29]

Fatigue (Vitality subscale of SF36 [30,31]

Health care utilization

$X$

Pregnancy Test (for premenopausal women)

$x$

ALT

Serum creatinine and eGFR

$X$

Blood Sodium

$x$

Contact sheet $\quad X$

GP Letter

$x$

Participant letter [central]

$\mathrm{X}$

\section{Outcome Measures}

\section{Primary Outcome}

The primary outcome is functional ability 180 days after randomisation, as measured by the modified Rankin Scale [mRS], using the simplified modified Rankin Scale questionnaire [smRSq 44, 45]. The mRS is a simple, reliable and valid 6-point measure of functional outcome commonly used as the primary outcome in most stroke treatment trials [44, 45]. The mRS is measured at baseline [pre and post stroke scores are recorded], 28, 90, 180 and 365 day assessments.

The mRS is derived from the smRSq assessment which consists of the following five questions:

1. Could you live alone without any help from another person? This means being able to bathe, use the toilet, shop, prepare or get meals, and manage finances

2. Are you able to do everything you were doing right before your stroke, even if slower and not as much?

3. Are you able to walk (from one room to another) without help from another person?

4. Are you completely back to the way you were right before your stroke?

5. Are you bedridden or needing constant supervision?

The mRS is scored as follows:

0 no symptoms;

1 no significant disability despite symptoms, able to carry out all usual duties and activities;

2 slight disability, unable to carry out all previous activities but able to look after own affairs without assistance;

3 moderate disability requiring some help, but able to walk without assistance;

4 moderate-severe disability, unable to walk without assistance and unable to attend to own bodily needs without assistance;

5 severe disability, bedridden, incontinent and requiring constant nursing care and attention;

6 dead.

\section{Secondary Outcomes}

Secondary outcome measures, recorded at follow-up assessments 28, 90, 180 and 365 days after randomisation are:

- Survival

Survival is recorded at the $28,90,180$ and 365 day assessments.

If the participant has died, details of the event including the date and cause of death are recorded as an outcome event on the SAE form. 
- $\underline{\text { Mood }}$

Mood is assessed at baseline, and at 28, 90, 180 and 365 days after randomisation, by completing the PHQ-9 [46], which scores each of the 9 Diagnostic and Statistical Manual of Mental Disorders, Fourth Edition (DSM-IV) criteria [58] as ' 0 ' (not at all) to ' 3 ' (nearly every day), generating a total score from $0-27$. Scores $\geq 15$ indicate significant depressive symptoms requiring treatment. The PHQ-9 has been validated for use in stroke patients and can generate a DSM-IV equivalent diagnosis [58].

- New diagnosis of depression requiring treatment;

A new clinical diagnosis of depression refers to, treatment for depression and prescription of an antidepressant since the previous assessment, therefore recorded at the 28, 90, 180 and 365 day assessments. This should refer to a clinical diagnosis made by a GP, psychologist, psychiatrist or hospital clinician since enrolment in the AFFINITY trial.

The responses to the following questions (which form part of the assessment of a new clinical diagnosis of depression at all follow up visits) should be completed before, and are independent of, the subsequent completion of the PHQ-9 and the associated score.

a. Has the patient been diagnosed with depression since the last assessment?

b. Has the patient been treated for depression since the last assessment? (nonpharmacological)

c. Has the patient been prescribed an antidepressant drug for treatment of depression since the last assessment?

Note: (b) Treatment non pharmacological can include psychotherapy, herbal remedies and other non-pharmacological therapies and electroconvulsive therapy.

- Fatigue

Fatigue is assessed at 180 and 365 day assessments by completing the vitality subscale of the full SF-36 [50,51].

- Cognitive function

Cognition is assessed at 180 and 365 day assessments using the TICSm, which scores 13 items (including orientation, recent and delayed memory, attention and comprehension) to a maximum possible score of 39 [47]. The TICSm has been validated in stroke patients and can be administered face-to-face or via telephone.

- Communication, motor function and overall Health Status

Communication, motor function, and overall health status is assessed at the 180 and 365 day assessments by the SIS [48], which measures 8 health domains (strength, hand function, activities of daily living/instrumental activities of daily living (ADL/IADL), mobility, communication, emotion, memory and thinking, participation). It has been evaluated successfully for use by proxy respondents [59].

- Health-Related Quality of Life (HRQoL)

Health related quality of life is assessed at the 180 and 365 day assessments by completing the EQ-5D-5L [49], a standardised instrument that provides a simple descriptive profile and a single index value for health status.

- Functional ability

The simplified modified Rankin Score (smRSq [44,45] is assessed at the 365 day assessment to ascertain if any beneficial effects of fluoxetine on recovery persist after it is stopped

Serious adverse events and secondary outcome events 
Assessed and recorded at the 28, 90, 180 and 365 day assessments.

- New Stroke, ischaemic or haemorrhagic [not the qualifying event leading to enrolment]

- Acute coronary syndrome [MI confirmed by ECG and/or raised serum Troponin]

- Upper gastrointestinal bleed requiring blood transfusion and/or endoscopy

- Other major bleed (i.e. not upper $\mathrm{Gl}$ or intracerebral) requiring blood transfusion or procedural intervention

- Fall

- New fracture [confirmed on X ray]

- Epileptic seizure [focal or generalised]

- Symptomatic hypoglycaemia [blood sugar $<3 \mathrm{mmol} / \mathrm{l}$ ]

- Symptomatic hyperglycaemia [blood sugar $>22 \mathrm{mmol} / \mathrm{l}$ ]

- New hyponatremia [ $\mathrm{Na}<125 \mathrm{mmol} / \mathrm{I}]$

- Attempted suicide/self-harm

- Death

- $\quad$ Health care utilisation

Assessed at the 180 and 365 day follow-ups by asking participants and using data linkage to provide information on hospital admissions, outpatient visits, days spent in care homes, health care visits and services utilised.

\section{Other Measures}

\section{Trial Medication Adherence}

Adherence is measured:

a) Subjectively, by response to the question 'On average, since the last follow up how many times per week was the trial medication taken? '0', '1-2', 3-4', '5-6' or '7' times per week (acknowledging that unintentional non-adherence is likely to be underestimated because some participants are unaware of their forgetfulness); and

b) Objectively, by pill counts and collection of returned trial bottles (acknowledging that absence of tablets in the bottle does not necessarily mean adherence to taking the tablets). Information on participants who temporarily or permanently stop the trial medication, and dates of and reasons for stopping are recorded. Analysis of medication adherence will compare groups based on the reported non-adherence and number of residual tablets.

\section{Trial Medication Cessation}

Medication cessation is recorded as temporary or permanent, together with dates and reasons for stopping.

\section{Concurrent antidepressant medication}

Participants are asked if they have been started on any antidepressant medication since enrolment (if yes, which drug(s) and indication).

\section{SERIOUS ADVERSE EVENTS/REACTIONS}

AFFINITY is evaluating fluoxetine, a very widely used SSRI that has been licensed for the treatment of depression since 1988 and used in thousands of patients with stroke to treat depression and emotionalism. Even though there is the potential for interactions of fluoxetine with medications frequently prescribed for stroke patients, such as aspirin and warfarin, these rarely cause significant problems. The trial materials given to the participant, and/or their carer will contain details of the known adverse reactions to fluoxetine $[60,61]$. 
A population-based cohort study of more than 60000 patients aged 65 years or more who were diagnosed with depression and followed up found that 764,650 prescriptions for SSRI antidepressants were issued and that, compared with when these drugs were not being used, SSRIs were associated with significantly higher rates of:

- all cause mortality ( $11.42 \%$ per year if taking fluoxteine vs $7.04 \%$ per year if not taking antidepressant; adjusted hazard ratio [HR]: 1.54, 95\% [Cl]: 1.48 to 1.59),

- stroke/transient ischaemic attack (2.57\% per year fluoxetine vs $2.23 \%$ per year no antidepressant; HR: 1.17, 1.10 to 1.26$)$,

- myocardial infarction (1.31\% vs $1.0 \%$ per year; HR: $1.15,1.04$ to 1.27$)$,

- upper gastrointestinal bleeding (0.48\% vs $0.42 \%$ per year; HR: $1.22,1.07$ to 1.40 ),

- falls (5.6\% vs $3.5 \%$ per year; HR: $1.66,1.58$ to 1.73$)$,

- fracture (2.76\% vs $1.76 \%$ per year; HR: $1.58,1.48$ to 1.68$)$,

- epilepsy/seizures (0.31\% vs $0.21 \%$ per year; HR: $1.83,1.49$ to 2.26$)$,

- attempted suicide/self-harm (0.53\% vs $0.25 \%$ per year; $2.16,1.71$ to 2.71 ), and

- hyponatraemia (0.49\% vs $0.29 \%$ per year; HR: $1.52,1.33$ to 1.75 ) [61].

Rates of most outcomes were highest in the first 28 days after starting an antidepressant, and also in the first 28 days after stopping.

The main concern with the above results is that they are based on observational studies and are therefore prone to residual confounding and indication bias [61]. Indication bias occurs when patients are prescribed drugs for a condition that is itself associated with the outcome of interest. This means that apparent associations with fluoxetine may be due to the condition for which it was prescribed (i.e. depression) rather than to the drug itself. Nevertheless, the above data are presented to indicate what could be causal adverse effects [61].

Irrespective of whether fluoxetine treatment is administered or not, about $20 \%$ of hospitalised patients with stroke would be expected to die in the first month after a stroke and another $10 \%$ by the end of the first year as part of the natural history of stroke. Up to a third will develop a chest or urinary infection whilst in hospital up to $5 \%$ may develop venous thromboembolism, epileptic seizures or gastrointestinal bleeding.

\section{Definitions}

An adverse event (AE) is any untoward medical occurrence in a clinical trial participant which does not necessarily have a causal relationship with an investigational medicinal product (IMP).

An $A E$ can therefore be any unfavourable or unintended sign (including an abnormal laboratory finding, for example), symptom or disease temporally associated with the use of a medicinal product, whether or not it is considered related to the medicinal product.

An adverse reaction (AR) is any untoward or unintended response to an IMP which is related to any dose administered to that participant.

An unexpected adverse drug reaction (UAR) is an adverse reaction, the nature or severity of which is not consistent with the applicable product information (e.g. investigator brochure for an unapproved investigational product or package insert/summary of product characteristic for an approved product).

The product information is provided and can be used as a guide to determine whether the event is expected or unexpected.

A serious adverse event (SAE), serious adverse reaction (SAR) or suspected unexpected serious adverse reaction (SUSAR) is any AE, AR or UAR that, at any dose: 
- results in death;

- is life threatening (i.e. the participant was at risk of death at the time of the event; it does not refer to an event which hypothetically might have caused death if it were more severe);

- requires hospitalisation or prolongation of existing hospitalisation;

- results in persistent or significant disability or incapacity;

- results in a congenital anomaly or birth defect;

- results in a secondary outcome event for the AFFINITY Trial (New stroke, ischaemic or haemorrhagic [not the qualifying stroke leading to enrolment], Acute coronary syndrome [MI confirmed on ECG and/or raised serum Troponin], Upper gastrointestinal bleed requiring blood transfusion and/or endoscopy, Other major bleed (i.e. not upper $\mathrm{Gl}$ or intracerebral) requiring blood transfusion or procedural intervention, New fracture [confirmed on $X$ Ray], Epileptic seizure [focal or generalised], New hyponatraemia [ $\mathrm{Na}<125 \mathrm{mmol} / \mathrm{l}]$, Attempted suicide/self-harm).

To ensure no confusion or misunderstanding of the difference between the terms "serious" and "severe", which are not synonymous, the following note of clarification is provided:

The term "severe" is often used to describe the intensity (severity) of a specific event (as in mild, moderate, or severe myocardial infarction); the event itself, however, may be of relatively minor medical significance (such as severe headache). This is not the same as "serious," which is based on patient/event outcome or action criteria usually associated with events that pose a threat to a participant's life or functioning as defined in the bullet points above. Seriousness (not severity) serves as a guide for defining regulatory reporting obligations.

A hospitalisation is to be considered an SAE only if it is an official admission. In addition, a hospitalisation planned before the start of the study for a pre-existing condition that has not worsened does not constitute an SAE.

\section{Recording and Reporting}

All SAEs, SARs and SUSARs are recorded from the time of consent until the end of follow-up. SAEs, SARs and SUSARs will also be assessed at the 28, 90, 180 and 365 day assessments.

If the event is serious and/or related to the trial medication it must be reported to the trial coordinating centre immediately (within 24 hours) of the site investigator becoming aware of the event. This is done by completing the serious adverse event form [Hard Copy] and then entering the information on the web-based CRF. If for any reason the web -based system cannot be accessed, the site investigator will need to fax the completed hard copy to the trial coordinating centre.

The site investigator is also responsible for reporting any SAEs, SARs and SUSARs that their participants may experience to the Lead Human Research Ethics Committee (HREC) responsible for their site immediately (within 24hrs) of becoming aware of the event and according to their local guidelines.

SUSARs are reported according to regulatory requirements to the Therapeutic Goods Administration (TGA) in an expedited manner by the trial coordinating centre when a report is received from a site, the initial report is due within 7 calendar days of receipt of the event if the event is fatal or life threatening, with a follow up report within a further 8 days or within 15 calendar days of receipt of the event for non-fatal, non-life threatening events. 
The Data Monitoring Committee (DMC) will closely monitor the relative frequency of SAEs in the treatment and control groups and, in turn, will advise the steering committee of any concerns.

The trial coordinating centre will collate all SAEs, SARs and SUSARs that occur in the trial and submit 6 monthly reports to the lead HRECs as applicable. Site investigators will also receive copies of these reports and are responsible for submitting them to their local research governance office as per local guidelines.

Monitoring Side Effects and Interactions of fluoxetine

Participants are assessed 28 days after baseline to monitor for drug safety and SAEs and are clinically managed as appropriate. Any reported unexpected serious adverse events are discussed with one of the trial clinicians and the participant's GP. This information is used to guide a decision regarding their continuation on the trial medication. In addition, we will provide all participants and their GPs with a list of common serious adverse effects and drug interactions.

GPs are able to contact a trial clinician if any medication concerns arise during the trial.

\section{Depression}

Pre-existing (prevalent) depression is common amongst stroke patients.

Subsequent (incident) depression is also common after stroke, occurring in around a third of stroke survivors.

Depression may be detected during the AFFINITY trial:

1. At the baseline assessment, before randomisation by a score of 15 or greater on the PHQ-9 test.

2. During one of the AFFINITY trial follow-up assessments by a score of 15 or greater on the PHQ-9

3. During a visit to the participant's general practitioner (GP) or another clinician e.g. physician

After randomisation in the AFFINITY trial, participants are advised to inform their GP and any treating clinician that they are participating in a clinical trial and taking either fluoxetine $20 \mathrm{mg}$ or a placebo daily.

After randomisation, the site will send a letter to the GP of each participant. If there is no regular GP, participants are encouraged to nominate one. The letter provides information about the duration of the trial, medications to avoid due to potential interactions with fluoxetine, and suggestions on the management of a major depressive episode during the trial follow up period.

During the course of the trial, the AFFINITY trial coordinating centre will alert GPs if their patient displays moderate/severe depressive symptoms (PHQ-9 score $\geq 15$ ) during any follow-up assessments.

Some suggestions for managing depression in the AFFINITY trial are:

A. Prevalent (existing) depression: patients who are depressed before/at the time of randomisation.

1. If the patient is already taking an antidepressant medication and wishes to participate in the AFFINITY trial, the treating clinician should discuss with the patient and if possible the prescribing clinician whether or not it is possible and appropriate to discontinue the antidepressant (and perhaps start non-pharmacological treatment [e.g. clinical psychologist; see below] for the depression) before randomisation (to fluoxetine $20 \mathrm{mg}$, or placebo, daily) in the AFFINITY trial. 
Most antidepressants can be titrated down to zero over 5-7 days, but the treating clinician should check with their local pharmacy regarding specific guidelines for individual antidepressants. When the patient has been titrated off the current antidepressant they should then have a further 2-3 days free of medication before starting the AFFINITY trial medication.

2. If the patient is not being treated for depression but warrants it, and wants to participate in the AFFINITY trial, consider non-pharmacological treatment for the depression (i.e. referral to a clinical psychologist; see below) before randomisation in the AFFINITY trial.

Clinical psychology can be accessed through the Medicare Better Access initiative and is available to Australian residents and citizens. There is provision for up to 10 rebated sessions per year as part of a GP mental health treatment plan (http://www.health.gov.au/mentalhealth-betteraccess) .

B. Incident (new) depression: patients who develop depression after randomisation 0-6 months after randomisation

If the patient develops depression after randomisation in the AFFINITY trial and during the first 6 months of follow-up, whilst taking the AFFINITY trial medication (fluoxetine 20 $\mathrm{mg}$, or placebo, daily)

1. Consider non-pharmacological treatment for the depression if appropriate. such as advising an increase in social outlets, regular exercise or referral to a clinical psychologist.

1a. Clinical psychology can be accessed through the Medicare Better Access initiative and is available to Australian residents and citizens.

1b. There is provision for up to 10 rebated sessions per year as part of a GP mental health treatment plan (http://www.health.gov.au/mentalhealth-betteraccess).

2. If the treating clinician feels that antidepressant medication is necessary, consider the following:

2a. Commence treatment with an antidepressant that is NOT an SSRI, in addition to the AFFINITY trial medication.

The patient should be informed about the possibility of an interaction between the prescribed antidepressant and the AFFINITY trial drug (if fluoxetine), and the nature of symptoms of serotonin toxicity (which include confusion, sweating, unsteadiness, shaking, and diarrhoea) and hyponatraemia (confusion, seizures). The patient should be monitored regularly for the above symptoms and, if hyponatraemia is suspected, undergo a blood test for plasma sodium concentration.

2b. Consider referral to (or consultation with) a specialist psychiatrist.

6-12 months after randomisation

If the patient develops depression 6-12 months after randomisation in the AFFINITY trial, after having ceased the AFFINITY trial medication,

1. Consider non-pharmacological treatment for the depression if appropriate, such as advising an increase in social outlets, regular exercise or referral to a clinical psychologist.

1a. Clinical psychology can be accessed through the Medicare Better Access initiative and is available to Australian residents and citizens.

1b. There is provision for up to 10 rebated sessions per year as part of a GP mental health treatment plan (http://www.health.gov.au/mentalhealth-betteraccess). 
2. If the treating clinician feels that antidepressant medication is necessary, Consider the following:

2a. Commence treatment with an antidepressant that is NOT an SSRI

2b. Consider referral to (or consultation with) a specialist psychiatrist.

\section{STATISTICAL METHODS}

\section{Primary analysis}

The primary analysis will compare the mRS scores at the 180 day assessment for each treatment group by an ordinal logistic regression analysis, after adjusting for any baseline imbalance between the treatment groups in factors included in the minimisation algorithm $[62,63]$. The analysis will retain participants in the treatment groups they were originally allocated to $[64,65]$.

\section{Secondary analyses}

The main secondary analysis will compare, in each treatment group, at the 365 day assessment the mRS scores by means of an ordinal logistic regression analysis. Other secondary analyses will compare, in each treatment group, at 180 and 365 day assessments: the proportion of participants who are functionally independent according to the $\mathrm{mRS}$ score (0-2) by means of dichotomized outcome analysis, functional independence after controlling for survival (Cox proportional hazards model), mood (changes in PHQ-9 scores and proportion with PHQ-9 $\geq 15$ [46], cognition (TICSm scores [47]), communication (SIS [48]), motor function (SIS [48]), overall health status (SIS [48], HRQoL (EQ-5D-5L [49], new diagnosis of depression requiring treatment with antidepressants, fatigue (vitality domain of the SF-36 [50, 51]), medication adherence and cessation, and SAEs/SARs.

\section{Economic analyses}

An economic evaluation is conducted from the perspective of the health sector and will assess the incremental cost per Quality Adjusted Life Year (QALY) of the intervention strategy over placebo. Inpatient and outpatient costs, including the study drug, are estimated from each assessment and are costed using standard published rates (e.g. from Medical Benefits Schedule for non-hospital medical services, Pharmaceutical Benefits Schedule for prescribed medications and Australian Refined-Diagnostic Related Groups cost weights for hospital services). The average costs incurred by participants in both treatment groups are calculated.

Average HRQoL scores at the 365 day assessment for each participant are estimated and weighted by survival up to 365 days to determine a measure of QALY post-stroke. The average of this measure for participants in each treatment group is estimated to determine the incremental QALY gain/loss from the intervention and, when set alongside data on costs, will enable estimation of an incremental cost-effectiveness ratio. Sensitivity analysis will test uncertainty in key parameters (e.g. selection of cost weights and statistical variation in HRQoL scores). 


\section{Descriptive statistics}

For descriptive purposes baseline characteristics are presented by treatment groups. Discrete variables are summarised by frequencies and percentages, continuous variables by use of standard measures of central tendency and dispersion, mean and standard deviation (SD) or median and interquartile range (IQR). All variables are graphically analysed (i.e. box plots) to determine if variances between groups are equal and variables with unequal variances are transformed (e.g. log transformation) where necessary to ensure that any difference in coefficients are true differences.

\section{Sample size}

The AFFINITY trial will recruit 1600 participants over 4 years. Based on data from the Clots in Legs Or sTockings after Stroke (CLOTS) trial [66] and the Scandinavian Candesartan Acute Stroke Trial (SCAST) [67], we expect the proportion of participants not dependent on others (as indicated by a score of $0-2$ on the mRS [44]) to be $50 \%$ in the AFFINITY control group 180 days after randomisation (see Table 2).

Table 2. Expected distributions of smRSq scores at the 180 day assessment

\begin{tabular}{llllllll}
\hline smRSq score & $\mathbf{0}$ & $\mathbf{1}$ & $\mathbf{2}$ & $\mathbf{3}$ & $\mathbf{4}$ & $\mathbf{5}$ & $\mathbf{6}$ \\
\hline Control group & 0.10 & 0.20 & 0.20 & 0.15 & 0.10 & 0.10 & 0.15 \\
Intervention group & 0.13 & 0.24 & 0.21 & 0.14 & 0.09 & 0.08 & 0.12 \\
\hline
\end{tabular}

We expect that random assignment to fluoxetine will increase the odds of being functionally independent (mRS 0-2) at the 180 day assessment by 1.35 compared with control, which is a conservative estimate based on the effect of fluoxetine detected in the FLAME trial (OR = $3.8,95 \% \mathrm{Cl} 1.2$ to 10.7 ) [9]. An odds ratio of 1.35 is equivalent to an increase in the proportion of participants being functionally independent at the 180 day assessment by 7.5 percentage points (absolute increase; i.e. from $50 \%$ to $57.5 \%$ ) providing a clinically significant relative improvement of $15 \%$ (relative increase).

Assuming a common odds ratio of 1.35 in the ordinal logistic regression [68] and 90\% power, the trial will require 1600 participants taking into account the fact that up to $10 \%$ of living participants may be lost to follow-up.

With an effective sample size of at least 1440 participants completing follow-up, we will also be able to detect a mean difference in the PHQ-9 score of 1 with more than 90 percent power. This is based on a standard deviation of 5 as reported in other trials $[69,70]$.

The target of 1600 are reviewed by the blinded steering committee taking account accruing data on the distribution of participants (across both treatment groups) between different $\mathrm{mRS}$ categories and losses to follow up since these may influence the power of the trial. The steering committee would have the option of increasing the target for randomisation to maintain the trial power.

\section{ETHICS}

\section{Declaration of Helsinki, National Statement, International Conference on Harmonisation Good Clinical Practice (ICH-GCP) Guidelines}

The trial is performed in agreement with the Declaration of Helsinki and in accordance with relevant national and international regulatory and ethical frameworks. 


\section{Ethics review and approval}

It is the responsibility of the investigator at each participating site to obtain written approval from their relevant ethics committee and regulatory bodies before starting the trial. This documentation must be filed in the investigator site file (ISF) provided by the trial coordinating centre.

\section{Informed consent}

The informed consent procedures for this trial are listed below and should be followed according to local HREC guidelines which may differ between States and Territories in Australia and New Zealand.

The site investigator is responsible for ensuring informed consent is obtained from the participant before any protocol specific procedures are carried out.

Participants will receive oral and written information about the trial. The current ethics approved version of the participant information and consent form is provided for review to all potential participants. The oral explanation to the participant is performed by the site investigator or designated person, and covers all the elements specified in the participant information sheet and consent (PISC) form.

The participant is given every opportunity to clarify any points they do not understand and, if necessary, ask for more information. Sufficient time is given to the participant to consider the information provided and discuss the trial with their relatives if they wish.

The decision to participate in clinical research is voluntary and should be based on a clear understanding of what is involved.

Participants are asked to consent to being randomly allocated to fluoxetine or placebo capsules that are taken once daily for 180 days, in conjunction with four follow-up assessments over a total period of 365 days. The follow-up of participants has not been extended beyond the 365 day assessment at present. However it is possible that as the trial progresses and the results of the preliminary analyses become available, the steering committee may decide that it may be of scientific value to follow-up participants for five years. This would provide information on the possible beneficial long term treatment effects of fluoxetine. If this occurs we will notify the participant and ask for their consent to this extended follow-up.

The site investigator or delegated member of the trial team and the participant will sign and date the informed consent form to confirm that consent has been obtained. The original copy of the informed consent form is filed in the ISF for inspection by the trial monitor, a copy is given to the participant and a copy is filed in the participants medical records.

\section{Withdrawal of Consent}

Participants can withdraw from the trial at any time without loss of benefits to which they would otherwise be entitled. This process is clearly documented in the PISC form. All participant withdrawals need to be documented in the participant's medical records, on the withdrawal of consent form and entered onto the online web based system.

\section{Waiver of Consent/Next of Kin/Person responsible/Legal surrogate}

In Australia and New Zealand, the rules and system differ in every state regarding the appointment and powers of guardians, and consent to medical treatment by persons other than the participant. In all the states, the intention behind the legislation is the protection of the rights, welfare, freedom of decision and action, and interests of the person involved. 
The laws and definitions for the terms 'waiver of consent', 'next of kin', 'person responsible' and 'legal surrogate' in the context of a clinical trial may also differ between States and Territories in Australia and New Zealand, therefore the specific wording should be checked in each region and specific local ethics approval obtained.

\section{Data protection and retention}

All trial documentation is stored in a secure environment for a minimum of 15 years with all personal identifiers removed following trial closure, according to regulatory and ethical requirements.

\section{GOVERNANCE}

\section{Insurance and Indemnity}

Sites participating in the trial will seek insurance or indemnity to cover their liability from their local institution.

\section{QUALITY ASSURANCE}

\section{Source documents}

The purpose of source documents is to document the existence of the participant and substantiate the integrity of the trial data collected. The site investigator must maintain source documents for each participant in the trial. Source documents include original documents related to the trial, to medical treatment and the history of the participant. They can be hospital or clinic medical records, laboratory data and results of any other test or assessment.

Original PISC forms and the 28 and 90 day follow up CRF will be kept at the individual hospital site. Original consent forms must be filed in the ISF. 180 day and 365 day original CRF's will be kept at the trial coordinating centre.

Site personnel will be trained at site initiation on completion, collection and retention of adequate and accurate source documents. Source documents verify the authenticity of data recorded on the electronic CRFs and that the trial was carried out in accordance with the protocol. In the event of an on-site monitoring visit or a regulatory body audit these documents must be produced by the site for review.

At completion of the trial, the trial coordinating centre will confirm with the site that there are plans in place for the long-term storage of all the relevant data and source documentation (for a minimum of 15 years).

\section{Selection and monitoring of participating centres}

Potential centres are assessed by the trial coordinating centre to confirm they have adequate facilities and medical resources to conduct the trial. Prior to commencement of the trial at selected sites all designated research staff including the site investigator, co-site investigator(s) and Research Nurse(s) are trained in the methods of the trial at site initiation, which is held by teleconference.

Pharmacy specific initiations will be conducted via teleconference with pharmacy staff participating in the trial. This will include training on the protocol and all procedures and requirements for management of the trial medication; supply, storage, dispensing, returns, and all associated record keeping.

Prior to site initiation all the necessary ethical and regulatory approvals will be in place and copies of the associated documents will be held by the trial coordinating centre and filed on site in the investigator and pharmacy site files. The site investigator, co-site investigator(s), 
Research Nurse(s) and pharmacist (s) will sign and provide up-to-date Curriculum Vitaes (CVs) (maximum of two pages) to the trial coordinating centre along with a copy of the signed delegation of duties log.

Participating sites will be monitored using a risk based model, consisting of centralised monitoring of the eCRF by the trial coordinating centre and statistical data monitoring as follows:

\section{Centralised Monitoring of eCRF}

- Site acknowledgement that written informed consent (waiver of consent, next of kin, person responsible or legal surrogate if applicable) has been obtained from the participant according to the protocol is required to be documented for each participant on the source baseline assessment form which is then submitted on the eCRF.

- Participant information submitted on the eCRF will be screened for missing and inconsistent data. Queries will be raised and resolved directly with the site via the electronic interactive query report system, email and telephone.

- Participant follow up will be monitored continually for submission within the specified time parameters and contact made directly with the site if not received.

- Monitoring / Adjudication to a standard of $100 \%$ will be centrally managed for all reported: Serious Adverse Events, Protocol Violations, Pregnancy Notifications and Withdrawal of Consent

\section{Statistical Data Monitoring}

- The number of events by site as follows: serious adverse events, protocol violations, lost to follow up, withdrawn participants, will be monitored to identify unusally high or low numbers of reported events at individual sites.

- Unusually high numbers of late form submissions at individual sites.

- Unusually high numbers of missing data at individual sites.

Note: Unusual findings may flag the need for specific on site monitoring visits which will be carried out by the trial coordinating centre.

\section{Protocol deviations}

A protocol deviation is an unanticipated or unintentional departure from the expected conduct of an approved trial that is not consistent with the current protocol or consent document. A protocol deviation may be an omission, addition or change in any procedure described in the protocol

Except for changes to eliminate an immediate hazard to participants, the approved protocol is followed as specified. Protocol deviations are documented on a protocol violation form and entered onto the online data eCRF system, on submission of the form an email is sent to the trial coordinating centre. The hard copy is stored in the participant's site folder.

\section{Protocol amendments}

Any significant change in the trial protocol will require an amendment. Once the steering committee has approved a protocol amendment, it is the responsibility of the site investigator to submit this to each HREC for written approval. The approval letter, signed by the HREC chair, must refer specifically to the site investigator, the protocol number, the protocol title, the protocol amendment number, and the date of the protocol amendment. The protocol amendment may be implemented only after it has been approved by the HREC. A protocol change intended to eliminate an apparent immediate hazard to participants may be implemented immediately, but the change must then be documented in an amendment, reported to the HREC and the trial coordinating centre within 5 working days. 
If the revision is an administrative change (such as the addition or removal of committee members), a letter explaining the change(s) along with a copy of the amended pages(s) of the protocol must be submitted to the HREC for their information. No formal approval from the HREC is required prior to implementation of administrative changes.

\section{Responsibilities of the investigator at each participating site.}

It is the responsibility of the investigator at each participating site to:

- obtain written approval from the relevant ethics committee and regulatory bodies before starting the trial;

- ensure informed consent is obtained from the participant (or waiver of consent, next of kin, person responsible or legal surrogate) before any protocol specific procedures are carried out;

- ensure participants are managed in accordance with ICH-GCP guidelines;

- report any protocol violations and SAEs to their local hospital ethics committee and to the trial coordinating centre immediately and;

- report any protocol amendments, safety and DMC reports to their local hospital Ethics/regulatory bodies.

\section{Auditing by regulatory bodies}

The trial may be audited by inspectors appointed by government regulatory authorities. CRFs, medical records, source documents and other trial files must be accessible at all trial sites at times of auditing during the course of the trial and after the completion of the trial.

\section{Termination of the trial}

\section{Termination by the Steering Committee}

The trial management or steering committee may terminate the entire trial or terminate the trial at a particular centre at any time for any of the following reasons:

- Failure to enrol participants

- Protocol violations

- Inaccurate or incomplete data

- Unsafe or unethical practices

- Questionable safety of the treatment

- Suspected lack of efficacy of the treatment

- Lack of treatment safety

- Administrative decision

Trial withdrawal by a site investigator

If a site investigator withdraws from the trial prematurely, they are asked to:

$>$ Provide their local ethics committee, regulatory bodies and the trial coordinating centre with a written statement describing why they have withdrawn from the trial. (File a copy of this letter and acknowledgement received from the HREC in the ISF and send a copy to the trial coordinating centre via fax, email or mail).

$>$ Seek an alternative site investigator at the trial site to continue the management and follow-up of participants already randomised.

If this is not possible, and the site must close the site investigator is asked to:

$>$ Inform the steering committee, the trial coordinating centre and their local ethics committee and regulatory bodies of the decisions and reasons, and plans for ongoing management and follow-up of the participants.

$>$ Inform the trial participants of the decision and plans for their management and follow-up. 
Trial participants will be followed-up centrally by the trial coordinating centre if necessary.

\section{FUNDING}

The trial is funded by the National Health and Medical Research Council (NHMRC) project grant 1059094 for five years (2014-2018).

The start-up phase of the trial was funded during 2013 by NHMRC program grant 1013612, University of Sydney bridging support grant 2012-00055, and NHMRC Program grant 5711281.

\section{TRIAL ORGANISATION}

The AFFINITY trial is coordinated jointly by an established clinical trial team of independent investigators at Sir Charles Gairdner Hospital/University of Western Australia and the George Institute for Global Health in Sydney which has completed the NHMRC-funded VITAmins TO Prevent Stroke (VITATOPS) trial, led by Professor Graeme Hankey; and the Psychosocial Outcomes In StrokE (POISE) study, led by Associate Professor Maree Hackett. Both groups have well-developed systems and procedures for all aspects of the running of large-scale multi-centre clinical trials. Trial management is undertaken at Sir Charles Gairdner Hospital by a team led by Professor Graeme Hankey.

\section{Executive Steering Committee}

Associate Professor Maree Hackett, The George Institute for Global Health \& The University of Sydney (co-chair)

Professor Graeme J. Hankey, The University of Western Australia \& Sir Charles Gairdner Hospital (co-chair)

Professor Osvaldo Almeida, The University of Western Australia

Professor Craig S. Anderson, The George Institute for Global Health

Associate Professor Christopher Etherton-Beer, The University of Western Australia

Mr Laurent Billot, The George Institute for Global Health

Professor Martin S. Dennis, The University of Edinburgh

Professor Leon Flicker, Royal Perth Hospital

Associate Professor Andrew Ford, The University of Western Australia

Associate Professor Stephen Jan, The George Institute for Global Health

Professor Erik Lundström, Karolinska Institutet, Stockholm.

Professor Gillian Mead, The University of Edinburgh

Acknowledgement is made to the late Professor Veronica Murray, Danderyd Hospital Stockholm for her contributions to the trial as a founding steering committee member.

The steering committee carries the responsibility for the trial. Tasks include:

- Approval of the trial protocol and any amendments.

- Prevention, recognition and resolution of problems that may interfere with the conduct of the trial.

- Classification of outcome events on which no consensus is reached by the Auditing Committee.

- Deciding whether or not the trial should continue, based on the recommendations of the DMC.

- Writing manuscripts. 


\section{Principal Investigator Steering Committee}

All principal investigators at each site

\section{Data monitoring committee}

Data Monitoring Committee:

Professor Robert Hebert, Neuroscience Research Australia

Professor Gregory Carter, University of Newcastle \& Calvary Mater Newcastle Hospital

Professor Geoffrey Donnan, The Florey Institute of Neurosciences \& Mental Health

\section{Unblinded Statisticians:}

Associate Professor Qilong Yi, Canadian Blood Services and University of Toronto

Dr Qiang Li, The George Institute for Global Health, Sydney

An independent DMC is established to oversee the safety of participants in the trial. During the period of recruitment, interim analyses of baseline and follow up data is supplied, in strict confidence, to the chairperson of the DMC, along with any other analyses that the committee may request. In the light of these analyses, the DMC will advise the chairperson of the steering committee if, in their view, the randomised comparisons have provided both (i) 'proof beyond reasonable doubt' that for all, or some, the treatment is clearly indicated or clearly contra-indicated and (ii) evidence that might reasonably be expected to materially influence future patient management. Appropriate criteria of proof beyond reasonable doubt cannot be specified precisely, but the DMC will work on the principle that a difference of at least 3 standard errors in an interim analysis of a major outcome event (e.g. death from all causes or independent survival at the 180 day assessment) may be needed to justify halting, or modifying, a trial before the planned completed recruitment. This criterion has the practical advantage that the exact number of interim analyses would be of little importance, and so no fixed schedule is proposed. Following a report from the DMC, the steering committee will decide whether to modify entry to the trial (or seek extra data). Unless this happens however, the steering committee, the collaborators and central administrative staff will remain ignorant of the interim results.

Tasks include:

- Analyses of un-blinded interim data.

- Un-blinded analysis of SAEs.

- Formulation of recommendations to the steering committee on the continuation of the trial.

- Offering unsolicited recommendations to the steering committee, for example after publication of results of a similar trial.

\section{AFFINITY SUBSTUDIES}

After stroke, the brain undergoes spontaneous repair and remodelling. However, spontaneous recovery is variable and often incomplete. A better understanding of the mechanisms underlying recovery after stroke promises to advance restorative therapeutics.

Whilst the main AFFINITY trial will determine the overall efficacy of fluoxetine on functional recovery at 6 months after stroke (if it works), it does not address the mechanism of action of fluoxetine (how it works) and optimal patient selection (in whom it works).

A recent systematic review of antidepressants in animal models of ischaemic stroke reported that SSRIs reduced infarct volume by $27.3 \%$ (95\% Cl: $20.7 \%-33.8 \%$ ), increased neurogenesis by 2.2 SD (1.3-3.0), and improved neurobehavioral outcome by $51.8 \%$ (95\% Cl: $38.6 \%-$ $64.9 \%)[71]$. Fluoxetine may also have neuroprotective and other neurotrophic effects 
$[13,14,18,19]$ However, it remains uncertain whether the observed effect of fluoxetine in small trials, such as the FLAME trial [9], if real, may be mediated by augmented neuroprotection, neurogenesis, and neuroplasticity of brain networks, or mood.

The AFFINITY trial substudies aim to assess imaging and blood biomarkers of functional recovery after stroke and response to fluoxetine therapy.

\section{AFFINITY Imaging Biomarker study}

A recent meta-analysis of 24 functional magnetic resonance imaging (fMRI) studies of upper limb movement-related brain activity after stroke ( $n=255$ patients) reported that activity in ipsilesional primary motor and medial-premotor cortices after stroke indicated good motor recovery, whereas cerebellar vermis activity signalled poor recovery as compared to healthy controls [72]. Further, a recent review indicated that enhanced recovery can be linked to increased interhemispheric connectivity and increased grey matter volume (cortical thickness) in sensorimotor regions [73-76].

While studies of imaging for prediction of functional recovery after stroke are increasing $[77,78]$ few have analysed the effects of an intervention [73] particularly pharmacological interventions such as fluoxetine. A small clinical trial in 8 patients with stroke reported that fluoxetine was associated with hyperactivation in the ipsilesional primary motor cortex during an active motor task and significantly improved motor skills on the affected side compared with placebo $[79,80]$.

We hypothesize that response to treatment with fluoxetine (vs placebo) will be associated with between group differences in fMRI-based measures of task-related brain activation (specifically ipsilesional primary motor and medial-premotor cortices), enhanced functional connectivity (specifically interhemispheric connectivity) and increased brain volume (cortical thickness), in association with improved motor recovery and functional outcome at 6 months after stroke.

The primary aim of the AFFINITY MR imaging biomarker substudy, led by Professors Andrew Ford, Michael Bynevelt and Leanne Carey, is to determine if random allocation to fluoxetine is associated with

- $\quad$ increased magnitude of activation in the ipsilesional primary motor and medialpremotor cortices, as measured by task-related functional MRI (alternating fingertapping and rest paradigm) using the blood-oxygen-level dependent (BOLD) contrast $\mathrm{fMRI}$ technique, at 6 months after randomization, compared with placebo.

Secondary aims are to determine, at 6 months post-randomisation, if allocation to fluoxetine vs placebo is associated with MRI imaging evidence of:

- increased interhemispheric connectivity of sensorimotor cortices [resting state (RS) $\mathrm{fMRI}]$

- reduced disruption of functional connectivity of depression-related brain networks, including the thalamus (? mood mediation effect of fluoxetine) [RSfMRI] [81]

- $\quad$ greater volume and thickness of grey matter (cortical thickness) in the ipsilesional primary motor cortex, amygdala, and hippocampus (? neurogenesis) [high resolution structural 3-D T1-weighted MRI]

- $\quad$ greater white matter microstructural integrity and reduced loss of connectivity of white matter tracts [diffusion-weighted MRI (dMRI)] [82] 
- $\quad$ reduced volume of the qualifying stroke lesion [T2 fluid attenuation inversion recovery (FLAIR); automated lesion tracing/quantification] [83];

Changes in the above measures, between baseline and 6 months, in each treatment group will also be compared.

Participants recruited to the AFFINITY trial at the SCGH, RMH, John Hunter and Austin hospitals will be invited to undergo the standardised brief MRI protocol (T1WI [cortical thickness], brief dMRI [fractional anisotropy analysis of integrity of white matter tracts], and RS-fMRI [resting state functional connectivity]) immediately after randomization (before the first dose of study drug) and a more detailed MRI protocol (additional task-related fMRI and more detailed dMRI of connectivity) at 6 months after randomization and therapy.

A total of 80 participants (40 per group) will be required to reliably identify or exclude, with $95 \%$ power and a two-tailed alpha of 0.05 , a difference in magnitude of signal change in motor cortex activation associated with improved motor outcome, relative to usual care, based on effect size in our recent therapy intervention study [84]. In addition, analysis of data specific to modulation of cerebral activation with fluoxetine (Cohen's D of 1.50, power of $95 \%, p$ of 0.05 ), indicates a minimum sample size of 26 . We will recruit 100 participants in total for the AFFINITY FMRI study to allow for a dropout of up to $20 \%$ at 6 months.

\section{AFFINITY Blood Biomarker study}

Recovery after stroke is likely to be influenced by numerous biomarkers, but single biomarkers could also have important effects. Our preliminary analysis of molecular pathways associated with depression following mass spectrometry proteomic analysis in the START_PrePARE cohort study suggests the involvement of inflammatory, complement and coagulation systems. Further our meta-analysis of biomarkers of stroke-associated depressive illness revealed moderate effects for high cortisol levels and lower serum brainderived neurotrophic factor (BDNF) levels [85].

Other small studies suggest that polymorphisms in three genes related to neural repair may contribute to variability in functional outcome after stroke; the ApoE $\varepsilon 4$ polymorphism, the val66 met BDNF polymorphism, and the R0 mitochondrial DNA haplotype [86-88].

The AFFINITY blood biomarker substudy aims to explore the hypothesis that candidate blood biomarkers, identified through our meta-analyses [89] and PREPARE study [90], and genetic polymorphisms of the ApoE $\varepsilon 4$ and val66 met BDNF genes, the RO mitochondrial DNA haplotype, and other candidate markers of the neuroinflammatory, complement and coagulation systems (e.g. Gadd45b, compound 21, sigma 1 receptor, neurotrophin-3 [NT-3]), are associated independently and significantly with functional outcome after stroke and modulate the effect of fluoxetine on functional recovery. We will derive gene lists associated with good recovery for pathway and gene ontology analyses, and to assess pathways and mechanisms of action associated with treatment.

All participants enrolled in the AFFINITY trial will be invited to consent to provide $15 \mathrm{ml}$ of whole blood. $5 \mathrm{ml}$ will be placed in an EDTA anticoagulated tube and frozen $\left(-80^{\circ} \mathrm{C}\right)$ for later DNA extraction, and $10 \mathrm{ml}$ will be placed in a plain serum separator tube and frozen $\left(-80^{\circ} \mathrm{C}\right)$ for later separation into 2 separate aliquots (for multiple analyses) [91]. The blood will be stored at $-80^{\circ} \mathrm{C}$ at each investigator site. When 50 samples have been collected, they will be sent as a batch on dry ice to the central laboratory at PathWest, QEll medical centre, Perth for final storage and analysis at the completion of the study (Adj Prof John Beilby, biochemist and Dr. Mark Davis, molecular geneticist). Microarray analyses will be conducted for genesignature analysis and investigation of mechanisms associated with functional outcome [91]. 


\section{FOCUS TRIAL (UNITED KINGDOM) EFFECTS TRIAL (SWEDEN)}

The AFFINITY trial steering committee are collaborating with colleagues in the United Kingdom and Sweden who are concurrently running similar trials. (Fluoxetine or Control under Supervision, FOCUS) at the Department of Geriatric Medicine, Edinburgh and the Neurosciences Trial Unit in Edinburgh, United Kingdom and (Efficacy of fluoxetine-a randomised controlled trial in stroke, EFFECTS) at Karolinska Institutet, Stockholm. Both trials have similar eligibility criteria, treatments, outcome measures and follow-up schedule.

AFFINITY, FOCUS and EFFECTS steering committees plan to conduct a prospective metaanalysis which will enable us to identify smaller, but clinically important effect sizes, which neither trial could identify individually, and also allow us to identify clinically worthwhile effects in pre-specified subgroups. The committees have registered the review title with the Cochrane Stroke Group. The advantages of carrying out closely related but independent trials, rather than a single international trial are:

- Trial methods can match resources, infrastructure and regulations available in that country.

- Governmental funding agencies will only be asked to fund research activity in their country.

- Avoids the complexities of moving drug and placebo, and of dealing with indemnity, across international boundaries.

- That each trial will test the effect of fluoxetine in their particular environment e.g. with the intensity of therapy available in that country, with the background use of SSRIs etc to ensure that the result applies to patients in that country.

- That the health economic analyses are tailored to that country so that the implication for their health and social services can be deduced.

- The AFFINITY trial is able to explore secondary outcomes which will assist in elucidating possible mechanisms for any effects found.

- There is greater local ownership of the project and therefore hopefully improved recruitment and retention.

Concordant trial results that are statistically significantly positive would provide really reliable data that would be applicable to potentially millions of future patients a year.

\section{TRIAL TIMETABLE}

\section{Start-up phase}

A start-up phase, which aimed to randomise 200 patients in the first year to establish whether the protocol was feasible, was planned. This was to enable us to establish: a core trial management team, an IT system to manage web based randomisation, drug allocation, stock control, follow up, data collection and verification, and important aspects of feasibility including recruitment, medication adherence, questionnaire response and follow-up rates [all of which have been achieved].

Specifically, the start-up phase was to provide realistic estimates of:

1. the range of recruitment rates per hospital and thus the likely number of sites and duration of the main phase. It would also help identify barriers to recruitment which may allow us to increase recruitment rates.

2. the adherence rate, and reasons for non-adherence, which would influence our predicted effect size and power calculations.

3. the follow-up rate. 
Sites will be receiving a $\$ 1000$ payment per participant, payable to the site when all of the following are complete: Randomisation, 28 and 90 day follow-up assessment and submission to the trial coordinating centre by fax or email the participants verified contact details [participant contact sheet] for central follow-up at 6 and 12 months. Invoices will be raised on a quarterly basis, forms are considered complete when they have been submitted and any data queries raised have been answered satisfactorily.

Table 3. TIMELINES AND MILESTONES

\begin{tabular}{ll}
\hline Date & Milestone \\
\hline Feb-Jun 2011 & Protocol and case report form design \\
May 2011 & Source drug supply and matching placebo \& packaging \\
Nov 2011 & Apply to ethics committees \\
Nov 2011 & Database design commences \\
Aug 2012 & Start-up recruiting sites \\
Jan 2013 & First participant in trial \\
Jul 2013 & Six month assessment of first participant in trial \\
Jan 2014 & Twelve month assessment of first participant in trial \\
Dec 2017 & Completion of recruitment of 1600 participants \\
Dec 2018 & Completion of last twelve month assessment \\
Apr 2019 & Finalisation of database and data lock \\
May 2019 & Presentation and publication of main results \\
\hline
\end{tabular}

\section{Main phase}

The main trial will recruit a total of 1600 patients in order to have sufficient statistical power to detect differences in a primary outcome of $\mathrm{mRS}$ score for the entire group, and to detect differences in specific outcomes in pre-specified strata based on neurological deficits at baseline.

As it may not be feasible to enrol sufficient participants to reliably detect moderate effect sizes in these strata on our primary outcome (mRS) we will introduce two strategies:

1. Collect outcome measures which are likely to be more sensitive than our primary outcome to the possible benefits of fluoxetine in specific strata.

2. To work collaboratively with a parallel trial (FOCUS) based in the UK and (EFFECTS) based in Sweden, which has a similar design to increase the overall sample size and the numbers of participants in each of the important strata. We will perform prespecified meta-analyses to maximise our chances of detecting benefits in specific strata.

\section{PUBLICATION OF TRIAL RESULTS}

Ownership of the data arising from this trial resides with the trial team. The primary trial publication will be drafted by a writing committee whose membership has been approved by the steering committee. The manuscript must be approved by the steering committee before submission for publication. Planned publications will be submitted with an authorship list that states the names of the members of the Executive Steering Committee, Principal Investigator Steering Committee, Un-blinded Statisticians, and Trial Co-ordinating Centre. 


\section{REFERENCES}

1. Thrift AG, Dewey HM, Macdonell RA, McNeil JJ, Donnan GA. Stroke incidence on the east coast of Australia: the North East Melbourne Stroke Incidence Study (NEMESIS). Stroke. 2000; 31: 2087-92.

2. Islam MS, Anderson CS, Hankey GJ, Hardie K, Carter K, Broadhurst R, Jamrozik K. Trends in Incidence and Outcome of Stroke in Perth, Western Australia During 1989 to 2001. The Perth Community Stroke Study. Stroke. 2008; 39: 776-82.

3. Hackett M, Duncan J, Anderson C, Broad J, Bonita R. Health-related quality of life among long-term survivors of stroke: the Auckland Stroke Study. Stroke. 2000; 31: 440-447.

4. Hankey GJ, Jamrozik K, Broadhurst RJ, Forbes S, Anderson CS. Long-term disability after first-ever stroke and related prognostic factors in the Perth Community Stroke Study. Stroke 2002; 33: 1034-1040.

5. Sturm JW, Donnan GA, Dewey HM, Macdonell RA, Gilligan AK, Srikanth V, Thrift AG. Quality of life after stroke: the North East Melbourne Stroke Incidence Study (NEMESIS). Stroke. 2004; 35:2340-5.

6. Murray CJL, Vos T, Lozano R, et al. Disability-adjusted life years (DALYs) for 291 diseases and injuries in 21 regions, 1990-2010: a systematic analysis for the Global Burden of Disease Study 2010. Lancet 2012; 380: 2197-223.

7. Carod-Artal FJ, Egido JA. Quality of life after stroke: the importance of a good recovery.Cerebrovasc Dis. 2009;27 Suppl 1:204-14.

8. Langhorne $\mathrm{P}, \mathrm{Coupar} F$, Pollock A. Motor recovery after stroke: a systematic review. Lancet Neurol. 2009;8:741-54.

9. Chollet F, Tardy J, Albucher J-F, Thalamas C, Berard E, Lamy C, Bejot $Y$, Deltour S, Jaillard A, Niclot P, Guillon B, Moulin T, Marque P, Pariente J, Arnaud C, Loubinoux I. Fluoxetine for motor recovery after acute ischaemic stroke (FLAME): a randomised placebo-controlled trial. Lancet Neurology. 2011;10:123-130.

10. Jacobs BL, Fornal CA. Serotonin and motor activity. Curr Opin Neurobiol. 1997;7:820825

11. Jacobs BL. An animal behavior model for trialing central serotonergic synapses. Life Sci. 1976;19:777-785

12. Schmidt HD, Duman RS. The role of neurotrophic factors in adult hippocampal neurogenesis, antidepressant treatments and animal models of depressive-like behavior. Behav Pharmacol. 2007;18:391-418

13. Wang JW, David DJ, Monckton JE, Battaglia F, Hen R. Chronic fluoxetine stimulates maturation and synaptic plasticity of adult-born hippocampal granule cells. J Neurosci. 2008;28:1374-1384

14. Russo-Neustadt AA, Beard RC, Huang YM, Cotman CW. Physical activity and antidepressant treatment potentiate the expression of specific brain-derived neurotrophic factor transcripts in the rat hippocampus. Neuroscience. 2000;101:305312

15. Coppell AL, Pei Q, Zetterstrom TS. Bi-phasic change in BDNF gene expression following antidepressant drug treatment. Neuropharmacology. 2003;44:903-910

16. Nibuya M, Nestler EJ, Duman RS. Chronic antidepressant administration increases the expression of cAMP response element binding protein (CREB) in rat hippocampus. J Neurosci. 1996;16:2365-2372

17. Malberg JE, Eisch AJ, Nestler EJ, Duman RS. Chronic antidepressant treatment increases neurogenesis in adult rat hippocampus. J Neurosci. 2000;20:9104-9110 
18. Lim CM, Kim SW, Park JY, Kim C, Yoon SH, Lee JK. fluoxetine affords robust neuroprotection in the postischemic brain via its anti-inflammatory effect. J Neurosci Res. 2009;87:1037-1045

19. Shin TK, Kang MS, Lee HY, Seo MS, Kim SG, Kim CD, Lee WS. fluoxetine and sertraline attenuate postischemic brain injury in mice. Korean J Physiol Pharmacol. 2009;13:257-263

20. Loubinoux I, Boulanouar K, Ranjeva JP, Carel C, Berry I, Rascol O, Celsis P, Chollet F. Cerebral functional magnetic resonance imaging activation modulated by a single dose of the monoamine neurotransmission enhancers fluoxetine and fenozolone during hand sensorimotor tasks. J Cereb Blood Flow Metab. 1999;19:1365-1375

21. Pariente J, Loubinoux I, Carel C, Albucher JF, Leger A, Manelfe C, Rascol O, Chollet F. fluoxetine modulates motor performance and cerebral activation of patients recovering from stroke. Ann Neurol. 2001;50:718-729

22. Berends HI, Nijlant J, van Putten M, Movig KL, MJ IJ. Single dose of fluoxetine increases muscle activation in chronic stroke patients. Clin Neuropharmacol. 2009;32:1-5

23. Dam M, Tonin P, De Boni A, Pizzolato G, Casson S, Ermani M, Freo U, Piron L, Battistin L. Effects of fluoxetine and maprotiline on functional recovery in poststroke hemiplegic patients undergoing rehabilitation therapy. Stroke. 1996;27:1211-1214

24. Mahoney FI, Barthel DW. Functional evaluation: the Barthel Index. Maryland State Medical Journal. 1965;14:61-65

25. Acler M, Robol E, Fiaschi A, Manganotti P. A double blind placebo RCT to investigate the effects of serotonergic modulation on brain excitability and motor recovery in stroke patients. J Neurol. 2009;256:1152-1158

26. Mikami K, Jorge RE, Adams HP, al. e. Effect of antidepressants on the course of disability following stroke. American Journal of Geriatric Psychiatry. (in press)

27. 25. Marshall RS. Should every patient with stroke be on selective serotonin reuptake inhibitors?: No. Stroke. 2012; 43: 3152-3.

28. Mead GE, Hsieh CF, Lee R, Kutlubaev MA, Claxton A, Hankey GJ, Hackett ML. Selective serotonin reuptake inhibitors (SSRIs) for stroke recovery. Cochrane Database Syst Rev. 2012;11:CD009286. doi: 10.1002/14651858.CD009286.pub2.

29. Ferreira ML, Herbert RD, Crowther MJ, Verhagen A, Sutton AJ. When is a further clinical trial justified? BMJ. 2012; 345: e5913.

30. Mead GE, Dennis M, Lundström E, Murray V, Hackett M, Hankey GJ. Selective Serotonin Reuptake Inhibitors for Stroke: More Trials Are Needed. Stroke. 2013;44: e40-1.

31. Coupland C, Dhiman P, Morriss R, Arthur A, Barton G, Hippisley-Cox J. Antidepressant use and risk of adverse outcomes in older people: population based cohort. BMJ 2011;343:d4551.

32. Labos C, Dasgupta K, Nedjar H, Turecki G, Rahme E. Risk of bleeding associated with combined use of selective serotonin reuptake inhibitors and antiplatelet therapy following acute myocardial infarction. CMAJ. 2011; 183: 1835-43.

33. Schelleman $\mathrm{H}$, Brensinger CM, Bilker WB, Hennessy S. Antidepressant-warfarin interaction and associated gastrointestinal bleeding risk: a case-control study. PLoS One 2011;6: e21447.

34. Hackam DG, Mrkobrada M. Selective serotonin reuptake inhibitors and brain hemorrhage: a meta-analysis. Neurology. 2012; 79: 1862-5.

35. Mortensen JK, Larsson H, Johnsen SP, Andersen G. Post Stroke Use of Selective Serotonin Reuptake Inhibitors and Clinical Outcome Among Patients With Ischemic 
Stroke: A Nationwide Propensity Score-matched Follow-up Study. Stroke. 2013; 44: 420-6.

36. Scheidtmann K, Fries W, Muller F, Koenig E. Effect of levodopa in combination with physiotherapy on functional motor recovery after stroke: a prospective, randomised, doubleblind study. Lancet. 2001; 358: 787-790.

37. Martinsson L, Eksborg S, Wahlgren NG. Intensive early physiotherapy combined with dexamphetamine treatment in severe stroke: a randomized, controlled pilot study. Cerebrovasc Dis. 2003;16:338-345.

38. Sonde L, Lokk J. Effects of amphetamine and/or I-dopa and physiotherapy after stroke-a blinded randomized study. Acta Neurol Scand. 2007; 115: 55-59.

39. Prasad V, Gall V, Cifu A. The frequency of medical reversal. Arch Intern Med. 2011;171:1675- 1676.

40. Adams HP Jr, Robinson RG. Improving recovery after stroke: a role for antidepressant medications? Stroke. 2012; 43: 2829-32.

41. Chollet F. Selective serotonin reuptake inhibitors may be helpful in most patients with stroke. Stroke. 2012; 43:3150-1.

42. Selim MH, Molina CA. Poststroke treatment with selective serotonin reuptake inhibitors: a journey from sadness to motor recovery. Stroke. 2012; 43: 3154-5.

43. Robinson RG, Adams HP. Selective serotonin-reuptake inhibitors and recovery after stroke. Lancet Neurology. 2011;10: 110-111.

44. Bruno A, Shah N, Lin C, et al. Improving modified Rankin scale assessment with a simplified questionnaire. Stroke 2010; 41: 1048-50.

45. Dennis M, Mead G, Doubla F, Graham C. Determining the modified Rankin score after stroke by postal and telephone questionnaires. Stroke 2011 (submitted).

46. Williams LS, Brizendine EJ, Plue L, Bakas T, Tu W, Hendrie H, Kroenke K. Performance of the PHQ-9 as a screening tool for depression after stroke. Stroke. 2005;36:635638.

47. de Jager CA, Budge MM, Clarke R. Utility of TICS-M for the assessment of cognitive function in older adults. International Journal of Geriatric Psychiatry. 2003;18:318324

48. Edwards B, O'Connell B. Internal consistency and validity of the Stroke Impact Scale 2.0 (SIS 2.0) and SIS-16 in an Australian sample. Quality of Life Research. 2003;12:1127-1135

49. Herdman M, Gudex C, Lloyd A, Jannsen MF, Kind P, Parkin D, Bonsel G, Badia X. Development and preliminary testing of the new five-level version of EG-5D (EQ-5D5L). Quality of Life Research. Published online 9 April 2011.

50. Ware JE, Snow KK, Kosinski M, Gandek B. SF-36 health survey: manual and interpretation guide. Boston, MA: New England Medical Center, Health Institute; 1993.

51. Mead GE, JLynch J, Greig CA, Young A, Lewis S, Sharpe M. Evaluation of fatigue scales in stroke patients. Stroke. 2007; 38: 2090-2095.

52. Counsell C, Dennis M, McDowall M, Warlow C. Predicting outcome after acute stroke: development and validation of new models. Stroke 2002;33: 1041-7.

53. Generic Health. Fluoxetine. Product Information. Date of TGA Approval: 8 October 2008. Last updated on the TGA website: 1 November 2011.

54. Hatcher S, Arroll B. Newer antidepressants for the treatment of depression in adults. BMJ 2012; ;344:d8300.

55. Bamford J, Sandercock P, Dennis M, Warlow C, Jones L, McPherson K, Vessey M, Fowler G, Molyneux A, Hughes T, Burn J, Wade D. A prospective trial of acute 
cerebrovascular disease in the community: the Oxfordshire Community Stroke Project 1981-1986. Journal of Neurology, Neurosurgery \& Psychiatry. 1988;51:13731380

56. Adams HP, Bendixen BH, Kappelle LJ, Biller J, Love BB, Gordon DL, Marsh EE. Classification of subtype of acute ischemic stroke. Definitions for use in a multicenter clinical trial. TOAST. Trial of Org 10172 in Acute Stroke Treatment. Stroke. 1993;24:35-41

57. Brott TG, Adams HP, Olinger CP, Marler JR, Barsan WG, Biller J, Spilker J, Holleran R, Eberle R, Hertzberg V, Rorick M, Moomaw CJ, Walker M. Measurements of acute cerebral infarction: a clinical examination scale. Stroke. 1989;20:864-870.

58. American Psychiatric Association. Diagnostic and Statistical Manual of Mental Disorders: DSM-IV. Washington DC: American Psychiatric Association; 199

59. Duncan PW, Lai SM, Tyler D, Perera S, Reker DM, Studenski S. Evaluation of Proxy Responses to the Stroke Impact Scale. Stroke. 2002;33:2593-2599

60. Eli Lilly and Company. PROZAC (fluoxetine hydrochloride). Summary of Product Characteristics. 2009:Last updated on the eMC: 06/12/2010.

61. Coupland C, Dhiman P, Morriss R, Arthur A, Barton G, Hippisley-Cox J. Antidepressant use and risk of adverse outcomes in older people: population based cohort trial. BMJ 2011; 343: d4551

62. Saver JL. Novel end point analytic techniques and interpreting shifts across the entire range of outcome scales in acute stroke trials. Stroke. 2007;38:3055-3062

63. Savitz SI, Lew R, Bluhmki E, Hacke W, Fisher M. Shift analysis versus dichotomization of the modified Rankin Scale scores in the NINDS and ECASS-II trials. Stroke. 2007;38:3205-3012

64. Schulz KF, Altman DG, Moher D; CONSORT Group. CONSORT 2010 statement: updated guidelines for reporting parallel group randomised trials. BMJ. 2010;340:c332.

65. Altman DG. Missing outcomes in randomised trials: addressing the dilemma. Open Med 2009;3:e21-23.

66. The CLOTS Trials Collaboration. Effectiveness of thigh-length graduated compression stockings to reduce the risk of deep vein thrombosis after stroke (CLOTS trial 1): a multicentre, randomised controlled trial. Lancet. 2009;373:1958-1965

67. Sandset EC, Bath PMW, Boysen G, Jatuzis D, Kõrv J, Lüders S, Murray GD, Richter PS, Roine RO, Terént A, Thijs V, Berge E, on behalf of the SCAST Trial Group. The angiotensin-receptor blocker candesartan for treatment of acute stroke (SCAST): a randomised, placebo-controlled, double-blind trial. Lancet. 2011;377:741-750

68. Whitehead J. Sample size calculations for ordered categorical data. Statisstics in Medicine. 1993;12:2257-2271

69. Joubert J, Joubert L, Reid C, Barton D, Cumming T, Mitchell P, M. H, Heng R, Meadows G, Walterfang M, Pantelis C, Ames D, Davis S. The Positive Effect of Integrated Care on Depressive Symptoms in Stroke Survivors. Cerebrovascular Diseases. 2008;26:199-205

70. Löwe B, Schenkel I, Carney-Doebbeling C, Göbel C. Responsiveness of the PHQ-9 to psychopharmacological depression treatment. Psychosomatics. 2006;47:62-67.

71. McCann SK, Irvine C, Mead GE, Sena ES, Currie GL, Egan KE, Macleod MR, Howells DW. Efficacy of antidepressants in animal models of ischemic stroke: a systematic review and meta-analysis. Stroke. 2014; 45: 3055-63. 
72. Favre I, Zeffiro TA, Detante O, Krainik A, Hommel M, Jaillard A. Upper limb recovery after stroke is associated with ipsilesional primary motor cortical activity: a metaanalysis. Stroke. 2014; 45: 1077-83.

73. Carey LM et al. Beyond the lesion: neuroimaging foundations for post-stroke recovery. Future Neurology. 2013;8:507-27

74. Carter AR, Astafiev SV, Lang CE, et al. Resting interhemispheric functional magnetic resonance imaging connectivity predicts performance after stroke. Ann Neurol. 2010; 67: 365-75.

75. Carter AR, Shulman GL, Corbetta M. Why use a connectivity-based approach to study stroke and recovery of function? Neuroimage. 2012; 62: 2271-80.

76. Rehme AK, Grefkes C Cerebral network disorders after stroke: evidence from imaging-based connectivity analyses of active and resting brain states in humans. J Physiol. 2013; 591(Pt 1): 17-31.

77. Heiss WD, Kidwell CS, Imaging for prediction of functional outcome and assessment of recovery in ischaemic stroke. Stroke 2014; 45: 1195-1201.

78. Thiel A, Vahdat S. Structural and resting-state brain connectivity of motor networks after stroke. Stroke 2015; 46: 296-301.

79. Pariente J, Loubinoux I, Carel C, Albucher JF, Leger A, Manelfe C, Rascol O, Chollet F. Fluoxetine modulates motor performance and cerebral activation of patients recovering from stroke. Ann Neurol. 2001; 50: 718-29.

80. Loubinoux, I., Boulanouar, K., Ranjeva, J. P., Carel, C., Berry, I., Rascol, O., Celsis, P., and Chollet, $\mathrm{F}$. Cerebral functional magnetic resonance imaging activation modulated by a single dose of the monoamine neurotransmission enhancers fluoxetine and fenozolone during hand sensorimotor tasks. J. Cereb. Blood Flow Metab. 1999; 19: 1365-1375.

81. Palmer SM, Crewther SG, Carey LM and The START Project Team (2015) A metaanalysis of changes in brain activity in clinical depression. Front. Hum. Neurosci. 8:1045

82. Tournier, J. D., Yeh, C. H., Calamante, F., Cho, K. H., Connelly, A., \& Lin, C. P.. Resolving crossing fibres using constrained spherical deconvolution: validation using diffusion-weighted imaging phantom data. Neuroimage 2008; 42: 617-625.

83. Mitra, J., Bourgeat, P., Fripp, J., Ghose, S., Rose, S., Salvado, O., Connelly, A., Campbell, B., Palmer, S., Sharma, G., Christensen, S., Carey, L. Lesion segmentation from multimodal MRI using random forest following ischemic stroke. Neurolmage 2014; 98: 324-335.

84. Hubbard, I., Carey, LM., Budd, T. W. Levi, C, McElduff, P., Hudson, S., Bateman, G., Parsons, MW. A randomized controlled trial of early upper limb training on stroke recovery and brain activation. Neurorehabilitation and Neural Repair 2014; . pp. 1-11. ID NNR-14-0014. DOI: 10.1177/1545968314562647.

85. Noonan K, Carey L, Crewther S. Meta-analyses indicate associations between neuroendocrine activation, deactivation in neurotrophic and neuroimaging markers in depression after stroke. J Stroke Cerebrovasc Dis 2012; doi: 10.1016/j.jstrokecerebrovasdis.2012.09.008

86. Ploughman M, Windle V, MacLellan CL, White N, Doré JJ, Corbett D. Brain-derived neurotrophic factor contributes to recovery of skilled reaching after focal ischemia in rats. Stroke. 2009; 40:1490-5.

87. McHughen SA, Rodriguez PF, Kleim JA, Kleim ED, Marchal Crespo L, Procaccio V, Cramer SC. BDNF val66met polymorphism influences motor system function in the human brain. Cereb Cortex. 2010; 20: 1254-62. 
88. Cramer SC, Procaccio V; GAIN Americas; GAIN International Study Investigators. Correlation between genetic polymorphisms and stroke recovery: analysis of the GAIN Americas and GAIN International Studies. Eur J Neurol. 2012; 19: 718-24.

89. Warach, S., Pettigrew, L.C., Dashe, J.F., Pullicino, P., Lefkowitz, D.M., Sabounjian, L., Harnett, K., Schwiderski, U., Gammans, R. Effect of citicoline on ischemic lesions as measured by diffusion-weighted magnetic resonance imaging. Citicoline 010 Investigators. Ann Neurol 2000; 48: 713-722.

90. Carey, LM, Crewther, S, Salvado, O., ... Hankey GJ,..Davis, SM, Donnan, GA and the START research team. (2013) START (STroke imAging pRevention and Treatment): A longitudinal stroke cohort study: Clinical Trials Protocol. International Journal of Stroke. 2013 Nov 10. doi: 10.1111/ijs.12190

91. STroke imAging pRevention and Treatment (START) - PrePARE - Prediction and Prevention to Achieve Optimal Recovery Endpoints after stroke, Standalone protocol, V4.0. 26 Aug 2011; LABORATORY PROCEDURE MANUAL VERSION 1.0; 3 November 2011 (Protocol \# NTA0902). 
19. APPENDICES

APPENDIX 1

NATIONAL INSTITUTE OF HEALTH STROKE SCORE PG 53

APPENDIX 2 SIMPLIFIED MODIFIED RANKIN SCALE QUESTIONNAIRE ALGORITHM PG 58

APPENDIX 3

OXFORDSHIRE COMMUNITY STROKE PROJECT CLASSIFICATION OF CLINICAL STROKE SYNDROMES PG 59

APPENDIX 4 PATIENT HEALTH QUESTIONAIRE -9 ITEM PG 60

APPENDIX 5 TELEPHONE INTERVIEW FOR COGNITIVE STATUS -M PG 62

APPENDIX 6

SF-36 (VITALITY SUBSCALE) PG 63

APPENDIX 7 STROKE IMPACT SCALE PG 64

APPENDIX 8

EQ-5D-5L PG 70

APPENDIX 9

TRIAL MATERIALS PG 73

RANDOMISATION AND BASELINE FORM PG 73

28 DAY +/- 7 DAYS ASSESSMENT FORM PG 80

90 DAY +/- 7 DAYS ASSESSMENT FORM PG 84

180 DAY+/- 14 DAYS ASSESSMENT FORM PG 85 365 DAY +/- 14 DAYS ASSESSMENT FORM PG 101 
APPENDIX 1: NATIONAL INSTITUTE OF HEALTH STROKE SCORE

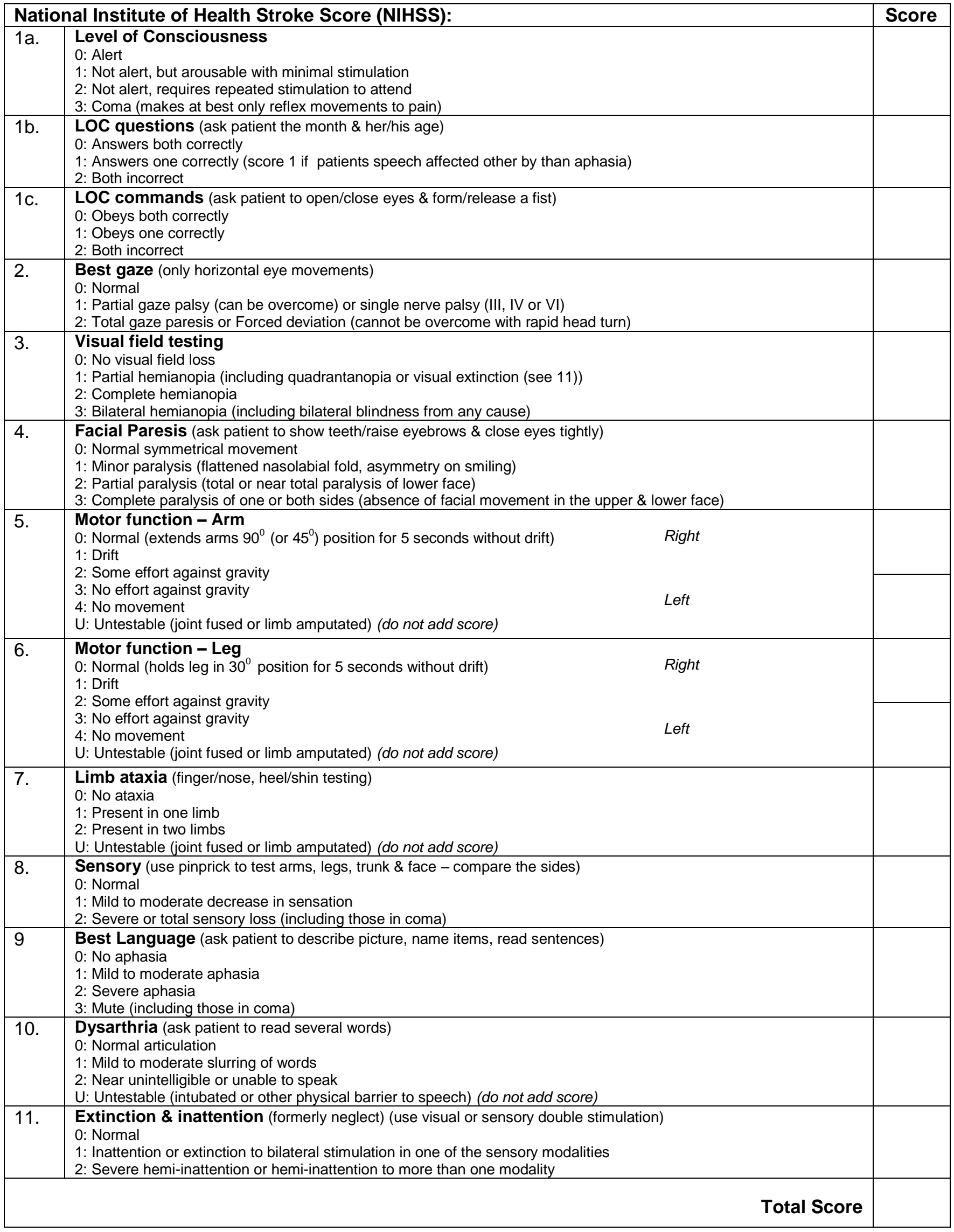




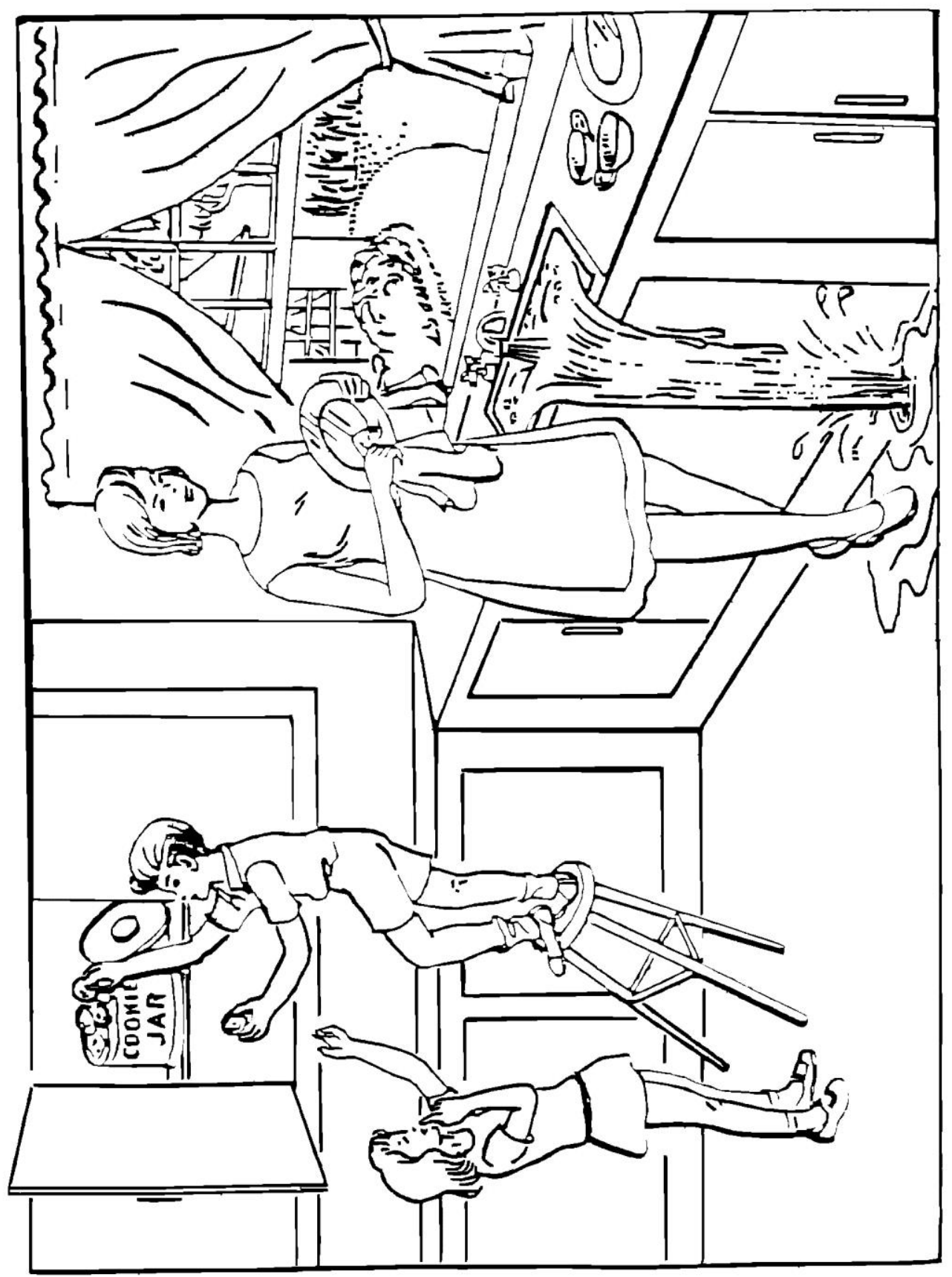




\title{
You know how.
}

\author{
Down to earth.
}

I got home from work.

Near the table in the dining room.

\section{They heard him speak on the radio last night.}




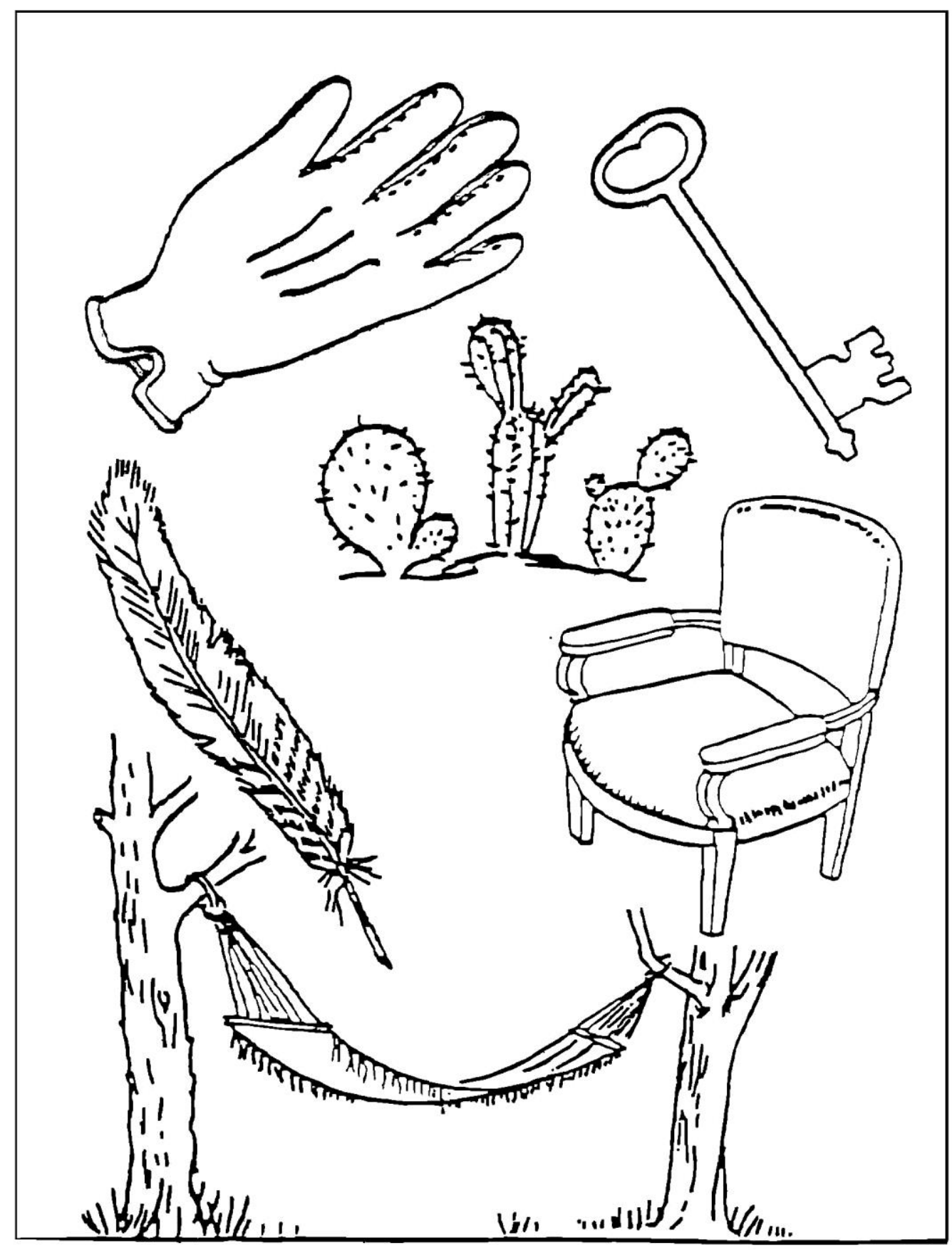




\section{MAMA \\ TIP - TOP \\ FIFTY - FIFTY \\ THANKS \\ HUCKLEBERRY}

\section{BASEBALL PLAYER}


Could you live alone without any help from another person? This means being able to bathe, use the toilet, shop, prepare or get meals, and manage finances.

Are you able to do everything that you were doing right before your stroke, even if slower and not as much?

2

Are you completely back to the way you were right before your stroke?

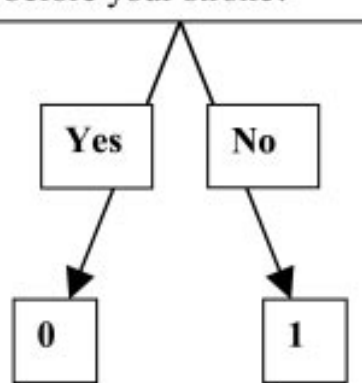

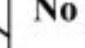

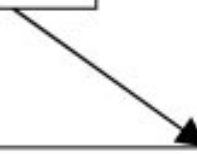

Are you able to walk without help from another person?

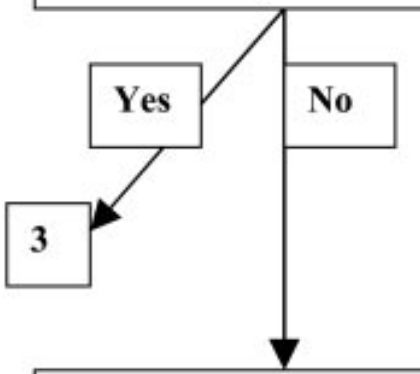

Are you bedridden or needing constant supervision?

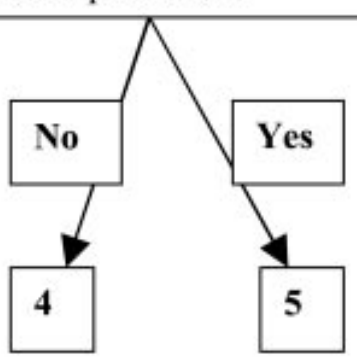




\section{APPENDIX 3: OXFORDSHIRE COMMUNITY STROKE PROJECT CLASSIFICIATION OF CLINICAL STROKE SYNDROMES}

\section{The Oxfordshire Community Stroke Project classification of clinical stroke}

syndromes

\section{Total anterior circulation syndromes}

- hemiparesis and homonymous hemianopia contralateral to the brain lesion, and

- either dysphasia or visuospatial perceptual disturbance

- \pm hemisensory deficit contralateral to the brain lesion.

\section{Partial anterior circulation syndrome}

- one or more of unilateral motor or sensory deficit, aphasia or visuospatial neglect (combined or not with homonymous hemianopia)

- motor or sensory deficit may be less extensive than in lacunar syndromes (for example, hand alone).

\section{Lacunar syndrome}

Any one of the following four syndromes involving at least two of the three areas (face, arm, leg), and involving the limb in its entirety:

- pure motor hemiparesis, or

- pure hemisensory deficit of one side of the body, or

- hemisensory-motor deficit, or

- ataxic hemiparesis (dysarthria clumsy hand syndrome or ipsilateral ataxia with crural hemiparesis)

- no visual field defect

- no new disturbance of higher cortical or brainstem function

\section{Posterior circulation syndromes}

Any one of:

- cranial nerve impairment

- unilateral or bilateral motor or sensory deficit

- disorder of conjugate eye movement

- cerebellar dysfunction

- homonymous hemianopia

- cortical blindness. 
APPENDIX 4: PATIENT HEALTH QUESTIONNAIRE - 9 ITEM

Fold back this page before administering this questionnaire

\section{INSTRUCTIONS FOR USE}

for doctor or healthcare professional use only

\section{PHQ-9 QUICK DEPRESSION ASSESSMENT}

For initial diagnosis:

1. Patient completes PHQ-9 Quick Depression Assessment on accompanying tear-off pad.

2. If there are at least $4 \sqrt{ } \mathrm{s}$ in the blue highlighted section (including Questions \#1 and \#2), consider a depressive disorder. Add score to determine severity.

3. Consider Major Depressive Disorder

-if there are at least $5 \sqrt{ } \mathrm{s}$ in the blue highlighted section (one of which corresponds to Question \#1 or \#2)

Consider Other Depressive Disorder

-if there are 2 to $4 \sqrt{ } \mathrm{s}$ in the blue highlighted section (one of which corresponds to Question \#1 or \#2)

Note: Since the questionnaire relies on patient self-report, all responses should be verified by the clinician and a definitive diagnosis made on clinical grounds, taking into account how well the patient understood the questionnaire, as well as other relevant

information from the patient. Diagnoses of Major Depressive Disorder or Other Depressive Disorder also require impairment of social, occupational, or other important areas of functioning (Question \#10) and ruling out normal bereavement, a history of a Manic

Episode (Bipolar Disorder), and a physical disorder, medication, or other drug as the biological cause of the depressive symptoms.

To monitor severity over time for newly diagnosed patients

or patients in current treatment for depression:

1. Patients may complete questionnaires at baseline and at regular intervals (eg, every 2 weeks) at home and bring them in at their next appointment for scoring or they may complete the questionnaire during each scheduled appointment.

2. Add up $\sqrt{ } \mathrm{s}$ by column. For every $\sqrt{ }$ : Several days $=1 \quad$ More than half the days $=2 \quad$ Nearly every day $=3$

3. Add together column scores to get a TOTAL score.

4. Refer to the accompanying PHQ-9 Scoring Card to interpret the TOTAL score.

5. Results may be included in patients' files to assist you in setting up a treatment goal, determining degree of response, as well as guiding treatment intervention.

\begin{tabular}{l} 
PHQ-9 SCORING CARD FOR SEVERITY DETERMINATION \\
for healthcare professional use only \\
Scoring - add up all checked boxes on PHQ-9 \\
For every $\checkmark$ : Not at all = 0; Several days = 1; \\
More than half the days = 2; Nearly every day = 3 \\
\hline Interpretation of Total Score \\
Total Score $\quad$ Depression Severity \\
$0-4$ None \\
$5-9$ Mild depression \\
$10-14$ Moderate depression \\
$15-19$ Moderately severe depression \\
$20-27$ Severe depression
\end{tabular}


NAME:

DATE:

Over the last 2 weeks, how often have you been bothered by any of the following problems? (use " $\checkmark$ " to indicate your answer)

1. Little interest or pleasure in doing things

2. Feeling down, depressed, or hopeless

3. Trouble falling or staying asleep, or sleeping too much

4. Feeling tired or having little energy

5. Poor appetite or overeating

6. Feeling bad about yourself-or that you are a failure or have let yourself or your family down

7. Trouble concentrating on things, such as reading the newspaper or watching television

8. Moving or speaking so slowly that other people could have noticed. Or the opposite-being so fidgety or restless that you have been moving around a lot more than usual

9. Thoughts that you would be better off dead, or of hurting yourself in some way

\begin{tabular}{l} 
or of hurting yourself in some way \\
\hline add columns: \\
$\begin{array}{l}\text { (Healthcare professional: For interpretation of TOTAL, TOTAL: } \\
\text { please refer to accompanying scoring card.) }\end{array}$
\end{tabular}

10. If you checked off any problems, how difficult have these problems made it for you to do your work, take care of things at home, or get along with other people?

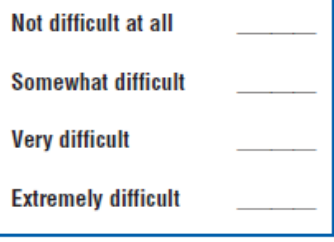

Extremely difficult

PHQ-9 is adapted from PRIME MD TODAY, developed by Drs Robert L. Spitzer, Janet B.W. Williams, Kurt Kroenke, and colleagues, with an educational grant from Pfizer Inc. For research information, contact Dr Spitzer at rls8@columbia.edu. Use of the PHQ-9 may only be made in accordance with the Terms of Use available at $h$ ttp://www.pfizer.com. Copyright 01999 Pfizer Inc. All rights reserved. PRIME MD TODAY is a trademark of Pfizer Inc.

ZT274388 


\title{
APPENDIX 5: TELEPHONE INTERVIEW FOR COGNITIVE STATUS - M
}

\section{APPENDIX}

Telephone Interview for Cognitive Status (TICS-M)

\section{Orientation}

1. (i) What day of the week is it?

(ii) What is today's date?

\author{
Day \\ Date \\ Month \\ Year \\ Season \\ Age:
}

(iii) What season are we in?

2. What is your age?

3 . What is your telephone number? (Code + number)

\section{Registration/Free Recall}

4. I'm going to read you a list of 10 words. Please listen carefully and try to remember them. When I am done, tell me as many as you can in any order. Ready?

Now, tell me all the words you can remember

\section{Attention/Calculation}

5. Please take 7 away from 100

Now continue to take 7 away from what you have left over until I ask you to stop.

6. Please count backwards from 20 to 1

\section{Comprehension, Semantic and Recent Memory}

7. What do people usually use to cut paper?

8 . What is the prickly green plant found in the desert?

9. Who is the reigning monarch now?

10. Who is the Prime Minister now?

11. What is the opposite of east?

\section{Language/Repetition}

12. Please say this 'Methodist Episcopal'

\section{Delayed Recall}

13. Please repeat the list of 10 words I read earlier

Score ' 1 ' for each correct answer and ' 0 ' if incorrect

Cabin

Pipe

Elephant

Chest

Silk

Theatre

Watch

Whip

Pillow

Giant

\section{3 \\ 86 \\ 79 \\ 72 \\ 65}

No mistakes

Scissors

Cactus

E, QE, QE2

Correct surname

West

Exactly right

Cabin

Pipe

Elephant

Chest

Silk

Theatre

Watch

Whip

Pillow

Giant 


\section{APPENDIX 6: SF-36 (VITALITY SUBSCALE)}

Q9a The following questions are about how you feel and how things have been with you in the past four weeks. As I read each statement, please give me the one answer that comes closest to the way you have been feeling. How much of the time during the past four weeks did you feel full of life?

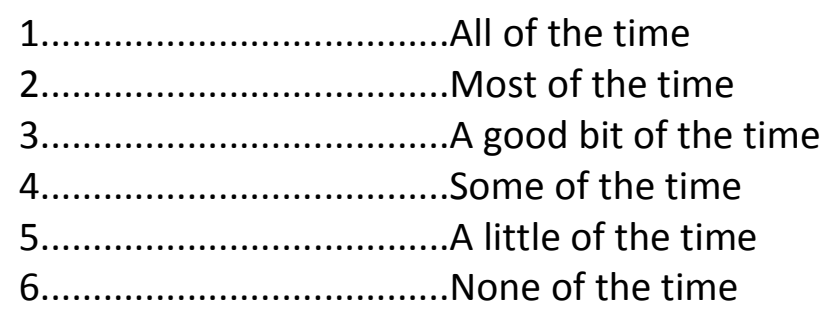

Q9e And how much of the time during the past four weeks did you have a lot of energy?
1 .....................................All of the time
2..................................... Most of the time
3...................................A good bit of the time
4...................................Some of the time

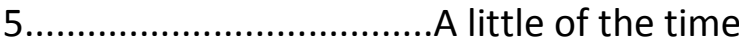
6.......................................None of the time

Q9g How much of the time during the past four weeks did you feel worn out?

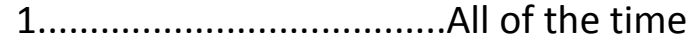

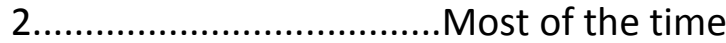
3................................... good bit of the time
4.................................. Some of the time

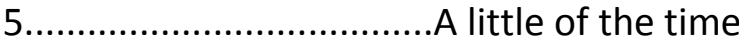
6......................................None of the time

Q9i How much of the time during the past four weeks did you feel tired?

the time




\section{APPENDIX 7: STROKE IMPACT SCALE \\ Stroke Impact Scale \\ VERSION 3.0}

The purpose of this questionnaire is to evaluate how stroke has impacted your health and life. We want to know from YOUR POINT OF VIEW how stroke has affected you. We will ask you questions about impairments and disabilities caused by your stroke, as well as how stroke has affected your quality of life. Finally, we will ask you to rate how much you think you have recovered from your stroke. 


\section{Stroke Impact Scale}

These questions are about the physical problems which may have occurred as a result of your stroke.

\begin{tabular}{|l|c|c|c|c|c|}
\hline $\begin{array}{l}\text { 1. In the past week, how would } \\
\text { you rate the strength of your.... }\end{array}$ & $\begin{array}{c}\text { A lot of } \\
\text { strength }\end{array}$ & $\begin{array}{c}\text { Quite a bit } \\
\text { of strength }\end{array}$ & $\begin{array}{c}\text { Some } \\
\text { strength }\end{array}$ & $\begin{array}{c}\text { A little } \\
\text { strength }\end{array}$ & $\begin{array}{c}\text { No } \\
\text { strength at } \\
\text { all }\end{array}$ \\
\hline $\begin{array}{l}\text { a. Arm that was most affected by } \\
\text { your stroke? }\end{array}$ & 5 & 4 & 3 & 2 & 1 \\
\hline $\begin{array}{l}\text { b. Grip of your hand that was } \\
\text { most affected by your stroke? }\end{array}$ & 5 & 4 & 3 & 2 & 1 \\
\hline $\begin{array}{l}\text { c. Leg that was most affected by } \\
\text { your stroke? }\end{array}$ & 5 & 4 & 3 & 2 & 1 \\
\hline $\begin{array}{l}\text { d. Foot/ankle that was most } \\
\text { affected by your stroke? }\end{array}$ & 5 & 4 & 3 & 2 & 1 \\
\hline
\end{tabular}

These questions are about your memory and thinking.

\begin{tabular}{|l|c|c|c|c|c|}
\hline $\begin{array}{l}\text { 2. In the past week, how difficult } \\
\text { was it for you to... }\end{array}$ & $\begin{array}{c}\text { Not } \\
\text { difficult at } \\
\text { all }\end{array}$ & $\begin{array}{c}\text { A little } \\
\text { difficult }\end{array}$ & $\begin{array}{c}\text { Somewhat } \\
\text { difficult }\end{array}$ & $\begin{array}{c}\text { Very } \\
\text { difficult }\end{array}$ & $\begin{array}{c}\text { Extremely } \\
\text { difficult }\end{array}$ \\
\hline $\begin{array}{l}\text { a. Remember things that people just } \\
\text { told you? }\end{array}$ & 5 & 4 & 3 & 2 & 1 \\
\hline $\begin{array}{l}\text { b. Remember things that happened the } \\
\text { day before? }\end{array}$ & 5 & 4 & 3 & 2 & 1 \\
\hline $\begin{array}{l}\text { c. Remember to do things (e.g. keep } \\
\text { scheduled appointments or take } \\
\text { medication)? }\end{array}$ & 5 & 4 & 3 & 2 & 1 \\
\hline d. Remember the day of the week? & 5 & 4 & 3 & 2 & 1 \\
\hline e. Concentrate? & 5 & 4 & 3 & 2 & 1 \\
\hline f. Think quickly? & 5 & 4 & 3 & 2 & 1 \\
\hline g. Solve everyday problems? & 5 & 4 & 3 & 2 & 1 \\
\hline
\end{tabular}


These questions are about how you feel, about changes in your mood and about your ability to control your emotions since your stroke.

\begin{tabular}{|l|c|c|c|c|c|}
\hline $\begin{array}{l}\text { 3. In the past week, how often did } \\
\text { you... }\end{array}$ & $\begin{array}{c}\text { None of } \\
\text { the time }\end{array}$ & $\begin{array}{c}\text { A little of } \\
\text { the time }\end{array}$ & $\begin{array}{c}\text { Some of } \\
\text { the time }\end{array}$ & $\begin{array}{c}\text { Most of } \\
\text { the time }\end{array}$ & $\begin{array}{c}\text { All of the } \\
\text { time }\end{array}$ \\
\hline a. Feel sad? & 5 & 4 & 3 & 2 & 1 \\
\hline $\begin{array}{l}\text { b. Feel that there is nobody you are } \\
\text { close to? }\end{array}$ & 5 & 4 & 3 & 2 & 1 \\
\hline c. Feel that you are a burden to others? & 5 & 4 & 3 & 2 & 1 \\
\hline $\begin{array}{l}\text { d. Feel that you have nothing to look } \\
\text { forward to? }\end{array}$ & 5 & 4 & 3 & 2 & 1 \\
\hline $\begin{array}{l}\text { e. Blame yourself for mistakes that } \\
\text { you made? }\end{array}$ & 5 & 4 & 3 & 2 & 1 \\
\hline $\begin{array}{l}\text { f. Enjoy things as much as ever? } \\
\text { g. Feel quite nervous? }\end{array}$ & 5 & 4 & 3 & 2 & 1 \\
\hline h. Feel that life is worth living? & 5 & 4 & 3 & 2 & 1 \\
\hline i. Smile and laugh at least once a day? & 5 & 4 & 3 & 2 & 1 \\
\hline
\end{tabular}

\section{The following questions are about your ability to communicate with other people, as well as your ability to understand what you read and what you hear in a conversation.}

\begin{tabular}{|l|c|c|c|c|c|}
\hline $\begin{array}{l}\text { 4. In the past week, how difficult } \\
\text { was it to... }\end{array}$ & $\begin{array}{c}\text { Not } \\
\text { difficult at } \\
\text { all }\end{array}$ & $\begin{array}{c}\text { A little } \\
\text { difficult }\end{array}$ & $\begin{array}{c}\text { Somewhat } \\
\text { difficult }\end{array}$ & $\begin{array}{c}\text { Very } \\
\text { difficult }\end{array}$ & $\begin{array}{c}\text { Extremely } \\
\text { difficult }\end{array}$ \\
\hline $\begin{array}{l}\text { a. Say the name of someone who was } \\
\text { in front of you? }\end{array}$ & 5 & 4 & 3 & 2 & 1 \\
\hline $\begin{array}{l}\text { b. Understand what was being said to } \\
\text { you in a conversation? }\end{array}$ & 5 & 4 & 3 & 2 & 1 \\
\hline $\begin{array}{l}\text { c. Reply to questions? } \\
\text { d. Correctly name objects? }\end{array}$ & 5 & 4 & 3 & 2 & 1 \\
\hline $\begin{array}{l}\text { e. Participate in a conversation with a } \\
\text { group of people? }\end{array}$ & 5 & 4 & 3 & 2 & 1 \\
\hline $\begin{array}{l}\text { f. Have a conversation on the } \\
\text { telephone? }\end{array}$ & 5 & 4 & 3 & 2 & 1 \\
\hline $\begin{array}{l}\text { g. Call another person on the } \\
\text { telephone, including selecting the } \\
\text { correct phone number and dialing? }\end{array}$ & 5 & 4 & 3 & 2 & 1 \\
\hline
\end{tabular}




\section{The following questions ask about activities you might do}

during a typical day.

\begin{tabular}{|l|c|c|c|c|c|}
\hline $\begin{array}{l}\text { 5. In the past 2 weeks, how difficult } \\
\text { was it to... }\end{array}$ & $\begin{array}{c}\text { Not difficult } \\
\text { at all }\end{array}$ & $\begin{array}{c}\text { A little } \\
\text { difficult }\end{array}$ & $\begin{array}{c}\text { Somewhat } \\
\text { difficult }\end{array}$ & $\begin{array}{c}\text { Very } \\
\text { difficult }\end{array}$ & $\begin{array}{c}\text { Could not } \\
\text { do at all }\end{array}$ \\
\hline a. Cut your food with a knife and fork? & 5 & 4 & 3 & 2 & 1 \\
\hline b. Dress the top part of your body? & 5 & 4 & 3 & 2 & 1 \\
\hline c. Bathe yourself? & 5 & 4 & 3 & 2 & 1 \\
\hline d. Clip your toenails? & 5 & 4 & 3 & 2 & 1 \\
\hline $\begin{array}{l}\text { e. Get to the toilet on time? } \\
\text { f. Control your bladder (not have an } \\
\text { accident)? }\end{array}$ & 5 & 4 & 3 & 2 & 1 \\
\hline $\begin{array}{l}\text { g. Control your bowels (not have an } \\
\text { accident)? }\end{array}$ & 5 & 4 & 3 & 2 & 1 \\
\hline $\begin{array}{l}\text { h. Do light household tasks/chores } \\
\text { (e.g. dust, make a bed, take out } \\
\text { garbage, do the dishes)? }\end{array}$ & 5 & 4 & 3 & 2 & 1 \\
\hline $\begin{array}{l}\text { i. Go shopping? } \\
\text { j. Do heavy household chores (e.g. } \\
\text { vacuum, laundry or yard work)? }\end{array}$ & 5 & 4 & 3 & 2 & 1 \\
\hline
\end{tabular}

The following questions are about your ability to be mobile, at home and in the community.

\begin{tabular}{|l|c|c|c|c|c|}
\hline $\begin{array}{l}\text { 6. In the past 2 weeks, how difficult } \\
\text { was it to... }\end{array}$ & $\begin{array}{c}\text { Not } \\
\text { difficult } \\
\text { at all }\end{array}$ & $\begin{array}{c}\text { A little } \\
\text { difficult }\end{array}$ & $\begin{array}{c}\text { Somewhat } \\
\text { difficult }\end{array}$ & $\begin{array}{c}\text { Very } \\
\text { difficult }\end{array}$ & $\begin{array}{c}\text { Could } \\
\text { not do at } \\
\text { all }\end{array}$ \\
\hline $\begin{array}{l}\text { a. Stay sitting without losing your } \\
\text { balance? }\end{array}$ & 5 & 4 & 3 & 2 & 1 \\
\hline $\begin{array}{l}\text { b. Stay standing without losing your } \\
\text { balance? }\end{array}$ & 5 & 4 & 3 & 2 & 1 \\
\hline c. Walk without losing your balance? & 5 & 4 & 3 & 2 & 1 \\
\hline d. Move from a bed to a chair? & 5 & 4 & 3 & 2 & 1 \\
\hline e. Walk one block? & 5 & 4 & 3 & 2 & 1 \\
\hline f. Walk fast? & 5 & 4 & 3 & 2 & 1 \\
\hline g. Climb one flight of stairs? & 5 & 4 & 3 & 2 & 1 \\
\hline h. Climb several flights of stairs? & 5 & 4 & 3 & 2 & 1 \\
\hline i. Get in and out of a car? & 5 & 4 & 3 & 2 & 1 \\
\hline
\end{tabular}


The following questions are about your ability to use your hand that was MOST AFFECTED by your stroke.

\begin{tabular}{|l|c|c|c|c|c|}
\hline $\begin{array}{l}\text { 7. In the past 2 weeks, how difficult } \\
\text { was it to use your hand that was most } \\
\text { affected by your stroke to... }\end{array}$ & $\begin{array}{c}\text { Not } \\
\text { difficult } \\
\text { at all }\end{array}$ & $\begin{array}{c}\text { A little } \\
\text { difficult }\end{array}$ & $\begin{array}{c}\text { Somewhat } \\
\text { difficult }\end{array}$ & $\begin{array}{c}\text { Very } \\
\text { difficult }\end{array}$ & $\begin{array}{c}\text { Could not } \\
\text { do at all }\end{array}$ \\
\hline $\begin{array}{l}\text { a. Carry heavy objects (e.g. bag of } \\
\text { groceries)? }\end{array}$ & 5 & 4 & 3 & 2 & 1 \\
\hline b. Turn a doorknob? & 5 & 4 & 3 & 2 & 1 \\
\hline c. Open a can or jar? & 5 & 4 & 3 & 2 & 1 \\
\hline d. Tie a shoe lace? & 5 & 4 & 3 & 2 & 1 \\
\hline e. Pick up a dime? & 5 & 4 & 3 & 2 & 1 \\
\hline
\end{tabular}

The following questions are about how stroke has affected your ability to participate in the activities that you usually do, things that are meaningful to you and help you to find purpose in life.

\begin{tabular}{|l|c|c|c|c|c|}
\hline $\begin{array}{l}\text { 8. During the past 4 weeks, how } \\
\text { much of the time have you been } \\
\text { limited in... }\end{array}$ & $\begin{array}{c}\text { None of } \\
\text { the time }\end{array}$ & $\begin{array}{c}\text { A little of } \\
\text { the time }\end{array}$ & $\begin{array}{c}\text { Some of } \\
\text { the time }\end{array}$ & $\begin{array}{c}\text { Most of } \\
\text { the time }\end{array}$ & $\begin{array}{c}\text { All of the } \\
\text { time }\end{array}$ \\
\hline a. Your work (paid, voluntary or other) & 5 & 4 & 3 & 2 & 1 \\
\hline b. Your social activities? & 5 & 4 & 3 & 2 & 1 \\
\hline c. Quiet recreation (crafts, reading)? & 5 & 4 & 3 & 2 & 1 \\
\hline $\begin{array}{l}\text { d. Active recreation (sports, outings, } \\
\text { travel)? }\end{array}$ & 5 & 4 & 3 & 2 & 1 \\
\hline $\begin{array}{l}\text { e. Your role as a family member } \\
\text { and/or friend? }\end{array}$ & 5 & 4 & 3 & 2 & 1 \\
\hline $\begin{array}{l}\text { f. Your participation in spiritual or } \\
\text { religious activities? }\end{array}$ & 5 & 4 & 3 & 2 & 1 \\
\hline $\begin{array}{l}\text { g. Your ability to control your life as } \\
\text { you wish? }\end{array}$ & 5 & 4 & 3 & 2 & 1 \\
\hline h. Your ability to help others? & 5 & 4 & 3 & 2 & 1 \\
\hline
\end{tabular}


9. Stroke Recovery

On a scale of 0 to 100 , with 100 representing full recovery and 0 representing no recovery, how much have you recovered from your stroke?

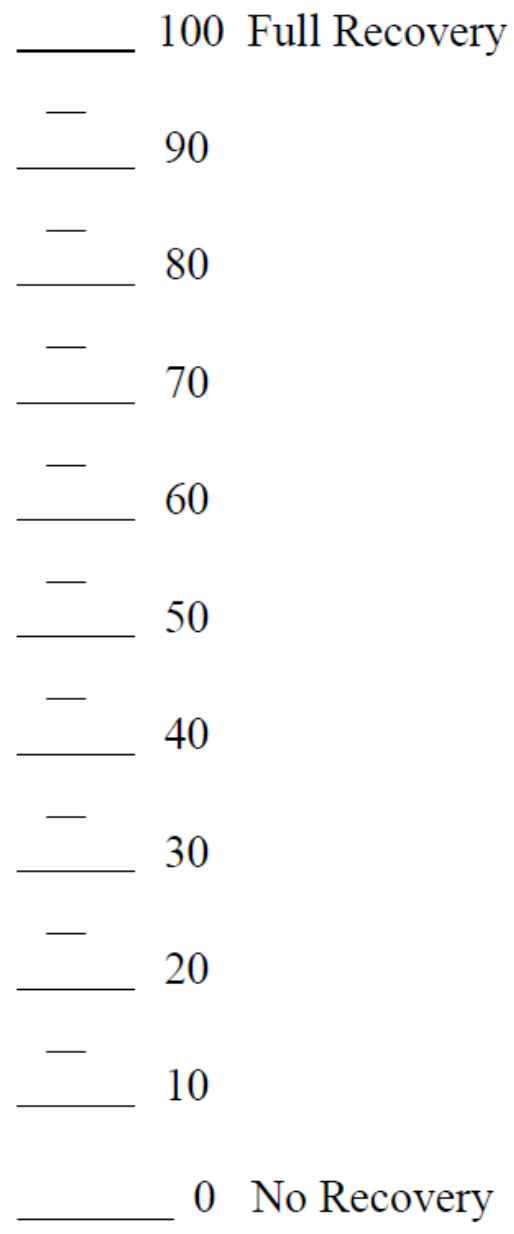




\section{(EO-5D-5L}

Health Questionnaire

\section{English version for Australia}


Under each heading, please tick the ONE box that best describes your health TODAY

\section{MOBILITY}

I have no problems with walking around

I have slight problems with walking around

I have moderate problems with walking around

I have severe problems with walking around

I am unable to walk around

\section{PERSONAL CARE}

I have no problems with washing or dressing myself

I have slight problems with washing or dressing myself

I have moderate problems with washing or dressing myself

I have severe problems with washing or dressing myself

I am unable to wash or dress myself

USUAL ACTIVITIES (e.g. work, study, housework, family or leisure activities)

I have no problems doing my usual activities

I have slight problems doing my usual activities

I have moderate problems doing my usual activities

I have severe problems doing my usual activities

I am unable to do my usual activities

\section{PAIN / DISCOMFORT}

I have no pain or discomfort

I have slight pain or discomfort

I have moderate pain or discomfort

I have severe pain or discomfort

I have extreme pain or discomfort

\section{ANXIETY / DEPRESSION}

I am not anxious or depressed

I am slightly anxious or depressed

I am moderately anxious or depressed

I am severely anxious or depressed

I am extremely anxious or depressed 
The best health

you can imagine

- We would like to know how good or bad your health is TODAY.

- This scale is numbered from 0 to 100 .

- 100 means the best health you can imagine. 0 means the worst health you can imagine.

- Mark an X on the scale to indicate how your health is TODAY.

- Now, please write the number you marked on the scale in the box below.
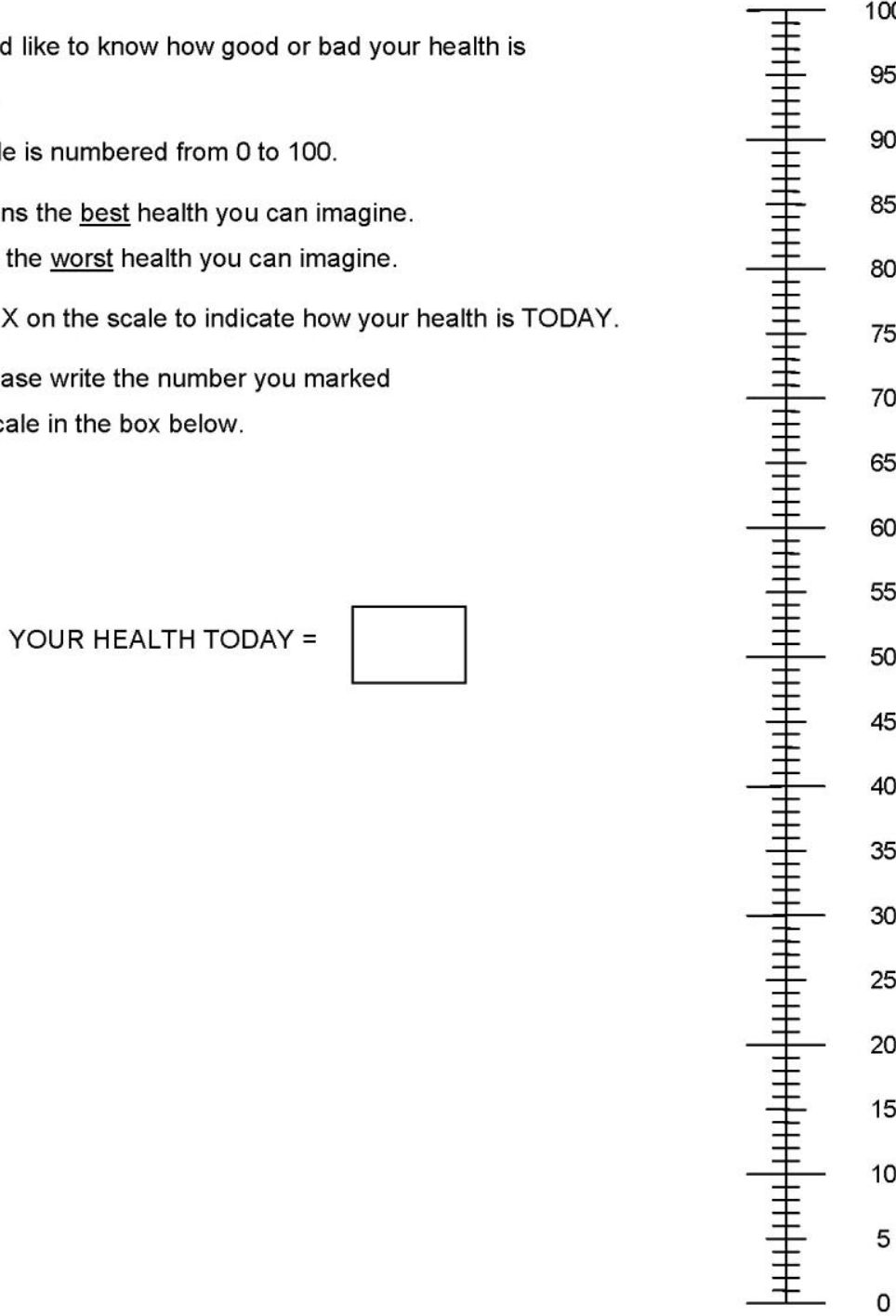

The worst health you can imagine 3

Australia (English) v.2 $\odot 2010$ EuroQol Group. EQ-5D TM is a trade mark of the EuroQol Group 
Please complete this baseline form on the day of randomisation, between 2 and 15 days post stroke

Randomisation MUST be done via the AFFINITY website www.affinitytrial.org

You MUST complete this form before logging on to the randomisation system

Date of Assessment: (dd/mm/yyyy) $\square \square / \square \square / \square \square \square \square \square$

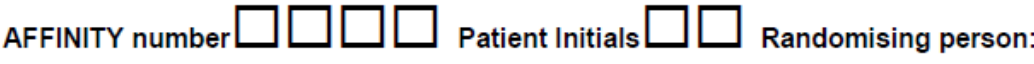

Collaborating centre: Consultant

1. Is the Patient Eligible?

Yes $\square \quad$ No

Inclusion criteria (check all criteria are met and all boxes ticked)

$\square \quad$ Men and Women aged $\geq 18$ years

$\square \quad$ Clinical diagnosis of stroke 2-15 days previously (Day of stroke onset = Day 0, randomise on Day 2-15)

$\square$ Brain imaging consistent with ischaemic or haemorrhagic (intracerebral and/or subarachnoid) stroke. (including normal CT brain scan)

$\square$ Persisting measurable focal neurological deficits (e.g. motor, somatosensory, visual, language, cognitive) present at randomisation and severe enough to produce a modified Rankin Scale (mRS) score of $\geq 1$ and to warrant treatment from the perspective of patient or carer(s).

Exclusion criteria (check no criteria are met and no boxes ticked)

$\square \quad$ History of epileptic seizures

$\square$ History of bipolar disorder

$\square$ History of drug overdose or attempted suicide

$\square \quad$ Ongoing treatment with any selective serotonin reuptake inhibitor (SSRI)

$\square$ Allergy or contra indication to fluoxetine including

$>$ hepatic impairment (serum alanine aminotransferase $[\mathrm{ALT}]>120 \mathrm{U} / \mathrm{l}$ ),

$>$ renal impairment (creatinine $>180 \mathrm{micromol} / \mathrm{l}$ or eGFR $<30 \mathrm{ml} / \mathrm{min} / 1.73 \mathrm{~m}^{2}$ ),

$>$ hyponatremia (sodium $<125 \mathrm{mmol} / \mathrm{L}$ ) despite treatment of the cause and confirmed on repeat testing,

$\square$ Use of medications that may interact seriously with fluoxetine

$>$ Proposed use of a monoamine oxidase inhibitor (MAOI), or use of a MAOI within 14 days prior to randomisation

$>$ Current treatment with an antipsychotic drug (neuroleptic), pimozide, tamoxifen, or tramadol unless the patient, doctor and if possible prescribing doctor, believe it is appropriate to discontinue use.

$\square \quad$ Not available for follow up over the next 365 days e.g. no fixed home address

$\square \quad$ Life-threatening illness (e.g. advanced cancer) that is likely to reduce 365 day survival

$\square$ Pregnant, breast-feeding or of child-bearing potential and not using contraception

$\square$ Enrolled in another interventional clinical research trial involving an investigational product (medicine) or device.

\section{Consent}

Has written consent been obtained from the patient or their legal representative? Yes $\square$ No $\square$

Given by: (please circle): $\quad$ Patient or Person Responsible or Waiver acknowledgement

Obtained by:

Date Obtained: (dd/mm/yyyy)

yyy) 


\section{Patient Demographics}

Gender:

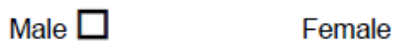

Date of Birth (dd/mm/yyyy):

$\square \square / \square \square / \square \square \square \square$

Ethnicity:

Caucasian

Asian

Aboriginal/Torres Strait Islander

African $\square$

Other $\square$ Please specify

Marital Status:

Married

Widowed

Partner

$\square$ Separated/Divorced

Other $\square$ Please specify

Living Arrangements before your stroke:

Living at home with someone else

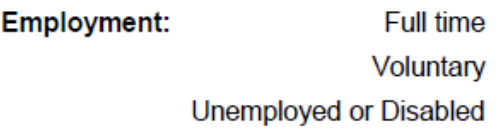

Home Alone $\square \quad$ Permanent care facility

Other $\square$ Please specify

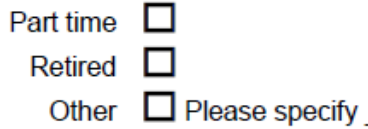

Is the patient currently an inpatient? $\quad$ Yes $\square \quad \mathrm{No} \square$

4. Functional status immediately before this stroke: (please circle)

0 No symptoms

1 No significant disability; despite symptoms; able to perform all usual duties and activities

2 Slight disability; unable to perform all previous activities but able to look after own affairs without assistance

3 Moderate disability; requires some help, but may be able to walk without assistance

4 Moderately severe disability; unable to walk without assistance and unable to attend to own bodily needs without assistance

5 Severe disability; bedridden, incontinent and requires constant nursing care and attention

\section{Details of Stroke}

Date of stroke: (dd/mm/yyyy) 
6. Neurological impairments (functional deficits):

National Institute of Health Stroke Score (NIHSS)

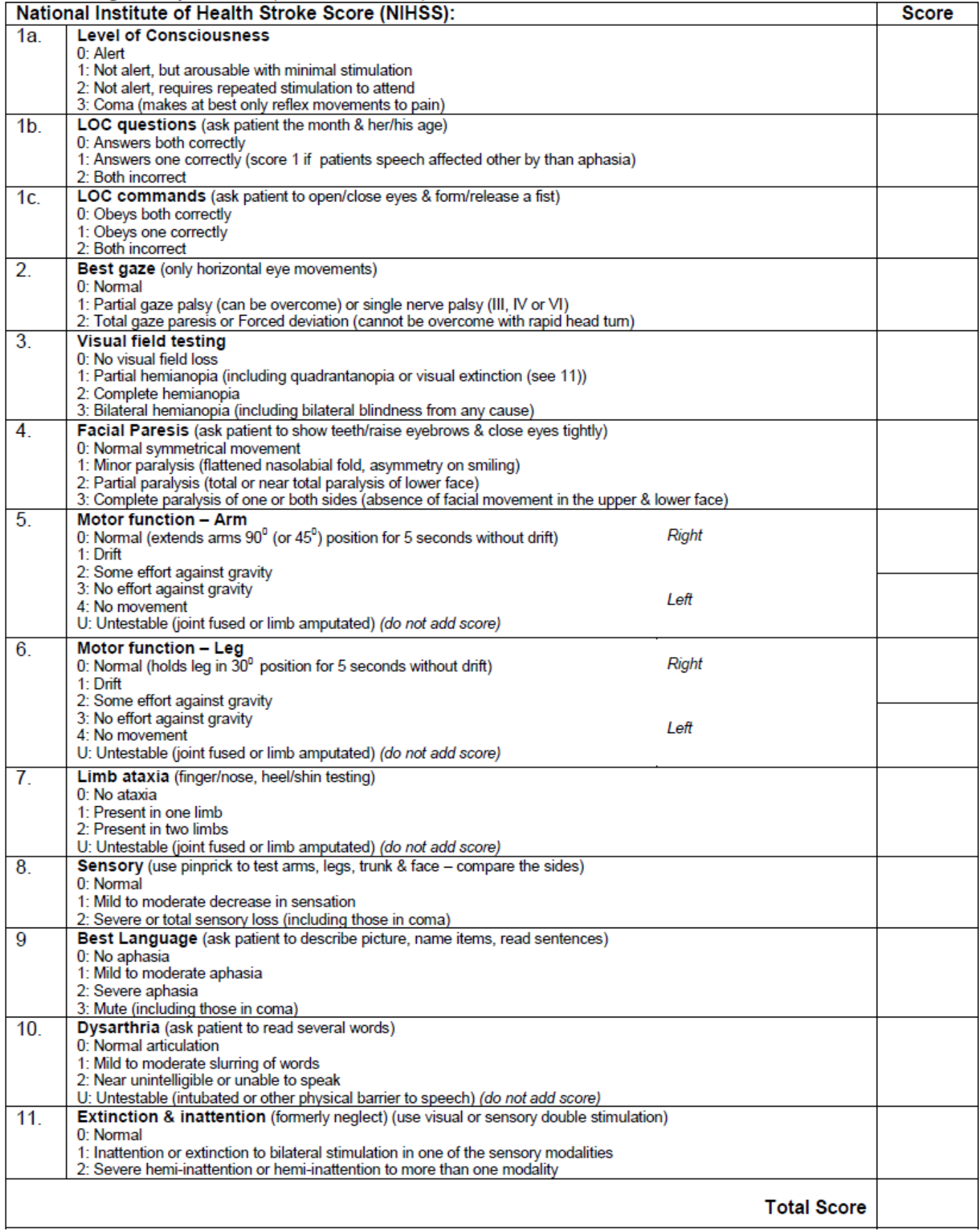




\begin{tabular}{|c|c|c|}
\hline i. & Able to lift both arms off the bed? & Yes $\square$ No $\square$ \\
\hline ii. & Able to walk (even with a walking aid) but without the help of another person & Yes $\square$ No $\square$ \\
\hline iii. & $\begin{array}{l}\text { Any other stroke deficit not captured by NIHSS? } \\
\text { If Yes, describe }\end{array}$ & Yes $\square$ No $\square$ \\
\hline
\end{tabular}

7. Primary Outcome: Simplified Modified Rankin Scale questionnaire (Circle each response on pathway \& final score)

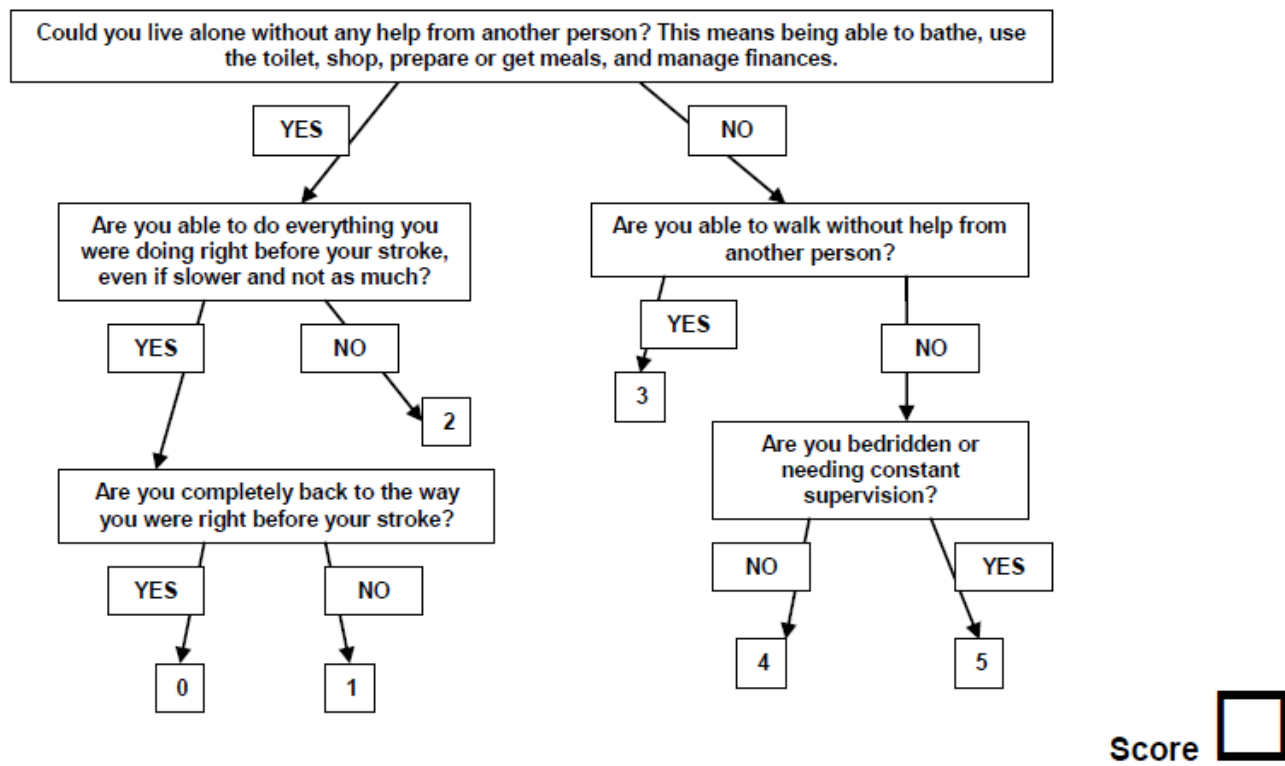

\section{Type of Stroke (Ischaemic or Haemorrhagic)}

Does brain scan show recent bleeding? Yes $\square$ No $\square$ (If no please answer 8a and 8b.)

If yes, is the bleeding likely to be due to haemorrhagic transformation of an infarct? Yes $\square$ No

If yes, please answer $8 \mathrm{a}$ and $8 \mathrm{~b}$

If no, please specify type of haemorrhage? i) Intracerebral haemorrhage $\square$ (please answer 8a and 8c.) ii) Subarachnoid haemorrhage $\square$ (please answer 8c.)

8a. What was the clinical syndrome of the qualifying stroke? (please tick most appropriate)
i. Total Anterior Circulation Syndrome (TACS)
ii. Partial Anterior Circulation Syndrome (PACS)
iii. LACunar Syndrome (LACS)
iv. Posterior Circulation Syndrome (POCS)
v. Uncertain 
8b. What is the most likely cause of the Ischaemic stroke? (please tick most likely)

i. Large artery disease (cortical stroke (TACS/PACS + arterial stenosis $>50 \%$ with no other cause)

ii. Small vessel disease (LACS without arterial stenosis or cardiac source)

iii. Embolism from the heart (e.g. atrial fibrillation, cardiomyopathy, endocarditis)

iv. Another cause (e.g. dissection, illicit drugs)

v. Unknown or uncertain cause (no cause identified or more than one of above)

$8 \mathrm{c}$. What is the likely cause of the haemorrhagic stroke? (please tick most likely)
i. Hypertension
ii. Amyloid angiopathy
iii. Antithrombotic therapy
iv. Arteriovenous malformation
v. Aneurysm
vi. Other,
vii. Unknown or uncertain

\section{Depression}

i. Previous depression requiring treatment? (Treatment can be Drugs/Counselling/other) Yes $\square$ No $\square$

ii. Current depression requiring treatment? (Treatment can be Drugs/Counselling/other) Yes $\square$ No $\square$

10. PHQ-9 (please circle) Over the past 2 weeks, have you been bothered by:

Scoring - $\quad 0$ : not at all; 1 : several days; 2 : more than half the days; 3 : nearly every day

\begin{tabular}{|c|l|c|c|c|c|}
\hline 1. & Little interest or pleasure in doing things? & 0 & 1 & 2 & 3 \\
\hline 2. & Feeling down, depressed, or hopeless? & 0 & 1 & 2 & 3 \\
\hline 3. & Trouble falling or staying asleep, or sleeping too much? & 0 & 1 & 2 & 3 \\
\hline 4. & Feeling tired or having little energy? & 0 & 1 & 2 & 3 \\
\hline 5. & Poor appetite or overeating? & 0 & 1 & 2 & 3 \\
\hline 6. & $\begin{array}{l}\text { Feeling bad about yourself- or that you are a failure or have let } \\
\text { yourself or your family down? }\end{array}$ & 0 & 1 & 2 & 3 \\
\hline 7. & $\begin{array}{l}\text { Trouble concentrating on things, such as reading the newspaper or } \\
\text { watching television? }\end{array}$ & 0 & 1 & 2 & 3 \\
\hline 8. & $\begin{array}{l}\text { Moving or speaking so slowly that other people could have noticed. } \\
\text { Or the opposite - being so fidgety or restless that you have been } \\
\text { moving around a lot more than usual? }\end{array}$ & 0 & 1 & 2 & 3 \\
\hline 9. & $\begin{array}{l}\text { Thoughts that you would be better off dead, or of hurting yourself in } \\
\text { some way? }\end{array}$ & 0 & 1 & 2 & 3 \\
\hline
\end{tabular}

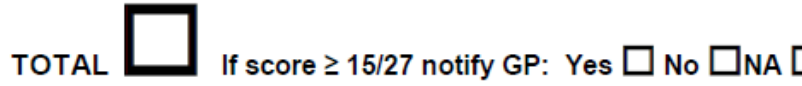

Not able to complete $\square$

10. If you have checked off any problems in the PHQ-9 above, how difficult have these problems made it for you to do your work, take care of things at home, or to get along with other people?

AFFINITY Randomisation Form Version $6,18^{\text {th }}$ November 2015
Not difficult at all $\square$ Somewhat difficult Very difficult $\square$ Extremely difficult 


\section{Co-morbidities}

\section{i. History of Diabetes}

Yes $\square$ No $\square$ Unknown $\square$

ii. Previous Coronary Heart Disease (ie definite angina, MI, CABG, coronary stenting)

iii. Previous ischaemic stroke/TIA or stroke of uncertain pathology (before this event)

iv. Previous Intracranial bleeding (including prior haemorhagic stroke or subdural)

v. Past history of upper gastrointestinal bleeding

vi. Past history of Hyponatraemia (Sodium $<125 \mathrm{mmol} /$ )

vii. Bone Fractures

\section{Was the patient thrombolysed for this event?}

13. Current Medications

Please list generic names of all medications being taken at the time of randomisation.

\begin{tabular}{|l|l|l|}
\hline 1. & 6. & 11. \\
\hline 2. & 7. & 12. \\
\hline 3. & 8. & 13. \\
\hline 4. & 9. & 14. \\
\hline 5. & 10. & 15. \\
\hline
\end{tabular}

\section{Laboratory Testing}

14a. ALT

Result: LU/L (if $>120 \mathrm{U} / \mathrm{L}$, please treat, repeat test and reassess before randomising)

14b. Creatinine

Result: $\square$ micromol/L (if > 180micromol/L please treat, repeat test and reassess before randomising)

14c. eGFR

Result: $\square$

(if $<30$, please treat, repeat test and reassess randomising)

14d. Blood sodium

Result:

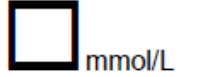

(if $<125 \mathrm{mmol} / \mathrm{L}$, please treat, repeat and reassess before randomising) 
15. Randomisation

To randomise this patient please log on to the AFFINITY trial website: www.affinitytrial.org

Please record the date of randomisation, patient ID and allocated treatment numbers below

Date of randomisation:(dd/mm/yyy) $\square \square / \square \square / \square \square \square \square$

AFFINITY trial patient ID number:

Allocated treatment number:
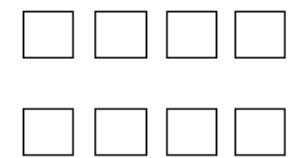

Medication Start Date: (dd/mm/yyyy)

Please write a prescription for a 6 month supply of AFFINITY trial medication.

\begin{tabular}{|c|c|c|}
\hline \multicolumn{3}{|c|}{ Rx AFFINITY trial medication (Fluoxetine $20 \mathrm{mg}$ or Placebo) } \\
\hline \multicolumn{3}{|c|}{ AFFINITY Treatment No. } \\
\hline Dose: & One capsule daily & \\
\hline Route: & Oral or NG/PEG (if cannot swallow) & \\
\hline Quantity: & 6 month supply & \\
\hline Name of $p$ & Signature: & Date: \\
\hline
\end{tabular}

Please make a follow-up appointment for the patient for 28 days from the randomisation date.

Please file the original copy of this randomisation form in the patient's AFFINITY folder and place a copy of the signed PISC in the patient's medical records

End of Baseline Assessment (thank you) 
Assessment Date: (dd/mm/yyyy)

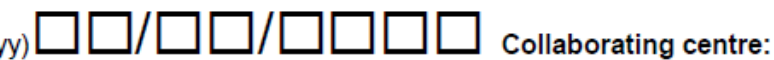

AFFINITY number $\square \square \square \square$ Patient Initials $\square \square$ Person completing this form:

Main source of the data: (please circle) Patient or Proxy or Medical Records or Other

1. Type of follow-up: (please circle)

Clinic Hospital Telephone Other (please list)

2. Survival: Is the patient alive at scheduled date of assessment? Yes $\square$ No $\square$ (If deceased, please complete a serious adverse event form.)

3. Current Living Arrangements: Home alone $\square$ Living at home with someone else $\square$ Permanent care facility Rehab Other $\square$ Please Specify

4. Clarification of the final diagnosis and cause of the qualifying stroke:

4a. Was the final diagnosis in this patient a definite stroke? Yes $\square$ No $\square$ (a normal brain scan is compatible with a diagnosis of ischaemic stroke)

If No, please specify the final diagnosis:

If Yes, what type of stroke was this?
i. Ischaemic
(please answer Q4c and d.)
ii. Haemorrhagic
$\square$ (please answer Q4b.)

4b. What type of haemorrhagic stroke?
i. Intracerebral haemorrhage
$\square$ (please answer Q4c and e.)
ii. Subarachnoid haemorrhage
$\square$ (please answer Q4e.)

4c. What was the clinical syndrome of the qualifying stroke?

i. Total Anterior Circulation Syndrome (TACS)

ii. Partial Anterior Circulation Syndrome (PACS)

iii. LACunar Syndrome (LACS)

iv. Posterior Circulation Syndrome (POCS)

v. Uncertain

4d. For patients with ischaemic stroke, what was the cause?

i. Large artery disease (cortical stroke (TACS/PACS + carotid atheroma $>50 \%$ with no other cause)

ii. Small vessel disease (LACS without carotid atheroma or cardiac source)

iii. Embolism from the heart (e.g. Atrial Fibrillation, prosthetic valve, endocarditis)

iv. Another cause (e.g. dissection, illicit drugs)

v. Unknown or uncertain cause (no cause identified or more than one of above)

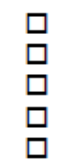

4e. What is the likely cause of the haemorrhagic stroke? (please tick most likely)
i. Hypertension
ii. Amyloid angiopathy
iii. Antithrombotic therapy
iv. Arteriovenous malformation
v. Aneurysm
vi. Other,
vii. Unknown or uncertain 
5. Current Medications: Please list all the medications the patient is currently taking

\begin{tabular}{|l|l|l|l|l|l|}
\hline \multicolumn{1}{|c|}{ Generic Name } & Start Date & Stop Date & Generic Name & Start Date & Stop Date \\
\hline 1. & & & 8. & & \\
\hline 2. & & & 9. & & \\
\hline 3. & & & 10. & & \\
\hline 4. & & & 11. & & \\
\hline 5. & & & 12. & & \\
\hline 6. & & & 13. & & \\
\hline 7. & & & 14. & & \\
\hline
\end{tabular}

6. Medication Compliance:

a. On average, since last follow up how many times per week was the AFFINITY trial medication taken (patient report)?
(0) times per week
(1-2) times per week
(3-4) times per week
(5-6) times per week
(7) times per week

b. How many capsules are remaining in the bottle?

c. Did patient stop the trial medication at all since the last follow up?

Yes, temporarily

$\square$ If Yes, specify dates and reason;

Yes, permanently

$\square$ If Yes, specify dates and reason;

No

\section{Date Stopped (dd/mm/yyy) $\square \square / \square \square / \square \square \square \square$ \\ Date Re-started (dd/mm/yyy) $\square \square / \square \square / \square \square \square \square$}

Number of days off AFFINITY medication

Reason:

7. Serious Adverse Events and/or Secondary Outcome Events since randomisation:

New stroke, ischaemic or haemorrhagic [not the qualifying stroke leading to enrolment] Yes

Acute coronary syndrome [MI confirmed on ECG and/or raised serum Troponin]

Upper gastrointestinal bleed requiring blood transfusion and/or endoscopy

Other major bleed (i.e. not upper Gl or intracerebral)

- Requiring blood transfusion or procedural intervention

Fall

New fracture [confirmed on X Ray]

Epileptic seizure [focal or generalised]

Symptomatic hypoglycaemia [blood sugar $<3 \mathrm{mmol} /$ ]

Symptomatic hyperglycaemia [blood sugar $>22 \mathrm{mmol} / \mathrm{l}]$

New hyponatraemia $[\mathrm{Na}<125 \mathrm{mmol} /]$

Attempted suicide/self-harm

Other SAE 
Please complete a "Serious Adverse Event and Secondary Outcome Event" form immediately if a patient has answered 'Yes' to any of the above events that:

- are unexpected reactions to the AFFINITY trial medication (i.e. not consistent with the product information)

- result in death;

- are life threatening (i.e. the participant was at risk of death due to the event; it does not refer to an event which hypothetically might have caused death if it were more severe);

- require hospitalisation or prolongation of existing hospitalisation;

- result in persistent or significant disability or incapacity;

- is a congenital anomaly or birth defect;

- results in a secondary outcome event for the AFFINITY Trial (new stroke, ischaemic or haemorrhagic [not the qualifying stroke leading to enrolment], acute coronary syndrome [MI confirmed on ECG and/or raised serum Troponin], upper gastrointestinal bleed requiring blood transfusion and/or endoscopy, other major bleed (i.e. not upper $\mathrm{Gl}$ or intracerebral) requiring blood transfusion or procedural intervention, new fracture [confirmed on X Ray], epileptic seizure [focal or generalised], new hyponatraemia [ $\mathrm{Na}<125 \mathrm{mmol} / \mathrm{l}$ or attempted suicide/self-harm)

\section{Pregnancy: Yes $\square$ No $\square$ N/A}

Please complete a separate 'pregnancy notification form'

\section{Primary Outcome}

9. Simplified Modified Rankin Scale questionnaire: (Circle each response on pathway \& final score)

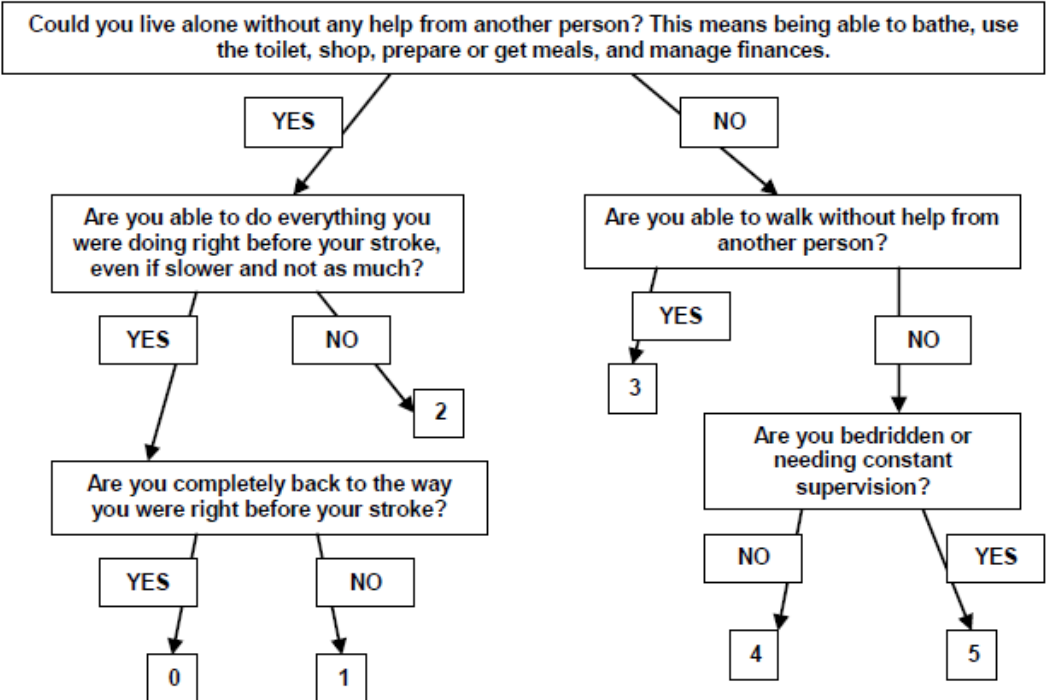




\section{Secondary Outcomes}

10. New clinical diagnosis of depression:

a. Has the patient been diagnosed with depression since randomisation?

b. Has the patient been treated for depression since randomisation? (non-pharmacological)

c. Has the patient been prescribed an antidepressant drug for treatment of depression since randomisation? Yes

If yes, to Q10c please ensure you have documented this on the current medication list.

11. PHQ-9 (please circle) Over the past 2 weeks, have you been bothered by:

Scoring - $\quad 0$ : not at all; 1 : several days; 2 : more than half the days; 3 : nearly every day

\begin{tabular}{|c|l|c|c|c|c|}
\hline 1. & Little interest or pleasure in doing things? & 0 & 1 & 2 & 3 \\
\hline 2. & Feeling down, depressed, or hopeless? & 0 & 1 & 2 & 3 \\
\hline 3. & Trouble falling or staying asleep, or sleeping too much? & 0 & 1 & 2 & 3 \\
\hline 4. & Feeling tired or having little energy? & 0 & 1 & 2 & 3 \\
\hline 5. & Poor appetite or overeating? & 0 & 1 & 2 & 3 \\
\hline 6. & $\begin{array}{l}\text { Feeling bad about yourself- or that you are a failure or have let } \\
\text { yourself or your family down? }\end{array}$ & 0 & 1 & 2 & 3 \\
\hline 7. & $\begin{array}{l}\text { Trouble concentrating on things, such as reading the } \\
\text { newspaper or watching television? }\end{array}$ & 0 & 1 & 2 & 3 \\
\hline 8. & $\begin{array}{l}\text { Moving or speaking so slowly that other people could have } \\
\text { noticed. Or the opposite - being so fidgety or restless that you } \\
\text { have been moving around a lot more than usual? }\end{array}$ & 0 & 1 & 2 & 3 \\
\hline 9. & $\begin{array}{l}\text { Thoughts that you would be better off dead, or of hurting } \\
\text { yourself in some way? }\end{array}$ & 0 & 1 & 2 & 3 \\
\hline
\end{tabular}

TOTAL $\square$ If score $\geq 15 / 27$ notify GP: Yes $\square$ No $\square$ NA $\square$

Not able to complete

10. If you have checked off any problems above, how difficult have these problems made it for you to do your work, take care of things at home, or get along with other people?

Not difficult at all $\square$ Somewhat difficult $\square$

Very difficult $\square$

Extremely difficult $\square$

Please enter this information onto the corresponding form via the AFFINITY website www.affinitytrial.org

End of 28 Day Assessment (thank you) 
AFFINITY Assessment of fluoxetine in stroke recovery

\section{AFFINITY trial 90 DAY +/- 14 days ASSESSMENT FORM}

Please use a black pen \& BLOCK PRINT IN CAPITALS

Assessment Date: (dd/mm/yyy) $\square \square / \square \square / \square \square \square \square$ collaborating centre:

\section{AFFINITY number $\square \square \square \square$ Patient Initials $\square \square$ Person completing this form}

Main source of the data (please circle): Patient or Proxy or Medical Records or Other

1. Type of follow-up: (please circle)
Clinic
Hospital
Telephone
Other (please list)

2. Survival: Is the patient alive at scheduled date of assessment? Yes $\square$ No $\square$

(If deceased, please complete a serious adverse event form.)

3. Current Living Arrangements: Home alone $\square$ Living at home with someone else $\square$ Permanent care facility Rehab $\square$ Other $\square$ Please Specify

4. Current Medications: Please list all the medications the patient is currently taking

\begin{tabular}{|l|l|l|l|l|l|}
\hline \multicolumn{1}{|c|}{ Generic Name } & Start Date & Stop Date & Generic Name & Start Date & Stop Date \\
\hline 1. & & & 8. & & \\
\hline 2. & & & 9. & & \\
\hline 3. & & & 10. & & \\
\hline 4. & & & 11. & & \\
\hline 5. & & & 12. & & \\
\hline 6. & & & 13. & & \\
\hline 7. & & & 14. & & \\
\hline
\end{tabular}

5. Medication Compliance

a. On average, since last follow up how many times per week was the AFFINITY trial medication taken (patient report)?
(0) times per week
(1-2) times per week
(3-4) times per week
(5-6) times per week
(7) times per week

b. How many capsules are remaining in the bottle?

c. Did patient stop the trial medication at all since the last follow up?

Yes, temporarily

If Yes, specify dates and reason;

Yes, permanently

$\square$ If Yes, specify dates and reason;

No

NA - permanently ceased reported on previous follow-up $\square$

Date Stopped (dd/mm/yyy) $\square \square / \square \square / \square \square \square \square$

Date Re-started (dd/mm/yyyy)

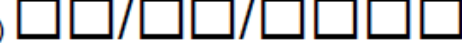

Number of days off AFFINITY medication:

Reason 
6. Serious Adverse Events and/or Secondary Outcome Events since 28 day follow-up: Yes $\square \quad$ No $\square$

New stroke, ischaemic or haemorrhagic [not the qualifying stroke leading to enrolment] Yes Acute coronary syndrome [confirmed on ECG and/or raised serum Troponin] Yes Upper gastrointestinal bleed requiring blood transfusion and/or endoscopy Yes Other major bleed (i.e. not upper $\mathrm{Gl}$ or intracerebral)

- Requiring blood transfusion or procedural intervention Fall

New fracture [confirmed on X Ray]

Epileptic seizure [focal or generalised]

Symptomatic hypoglycaemia [blood sugar $<3 \mathrm{mmol} / \mathrm{l}$ ]

Symptomatic hyperglycaemia [blood sugar $>22 \mathrm{mmol} / \mathrm{l}]$

New hyponatraemia $[\mathrm{Na}<125 \mathrm{mmol} / \mathrm{l}]$

Attempted suicide/self-harm

Other SAE

$\begin{array}{llll}\text { Yes } & \square & \text { No } & \square \\ \text { Yes } & \square & \text { No } & \square \\ \text { Yes } & \square & \text { No } & \square \\ \text { Yes } & \square & \text { No } & \square \\ \text { Yes } & \square & \text { No } & \square \\ \text { Yes } & \square & \text { No } & \square \\ \text { Yes } & \square & \text { No } & \square \\ \text { Yes } & \square & \text { No } & \square \\ \text { Yes } & \square & \text { No } & \square\end{array}$

Please complete a "Serious Adverse Event and Secondary Outcome Event" Form immediately if a patient has answered 'Yes" to any of the above events that:

- are unexpected reactions to the AFFINITY trial medication (i.e. not consistent with the product information)

- result in death;

- are life threatening (i.e. the participant was at risk of death due to the event; it does not refer to an event which hypothetically might have caused death if it were more severe);

- require hospitalisation or prolongation of existing hospitalisation;

- result in persistent or significant disability or incapacity;

- is a congenital anomaly or birth defect;

- results in a secondary outcome event for the AFFINITY Trial (new stroke, ischaemic or haemorrhagic [not the qualifying stroke leading to enrolment]. acute coronary syndrome [MI confirmed on ECG and/or raised serum troponin], upper gastrointestinal bleed requiring blood transfusion and/or endoscopy, other major bleed (i.e. not upper $\mathrm{Gl}$ or intracerebral) requiring blood transfusion or procedural intervention, new fracture [confirmed on X Ray], epileptic seizure [focal or generalised], new hyponatraemia $[\mathrm{NA}<125 \mathrm{mmol} / \mathrm{l}]$ or attempted suicide/self-harm).

7. Pregnancy: Yes

No

$\mathrm{N} / \mathrm{A}$

Please complete a separate 'pregnancy notification form' 


\section{Primary Outcome}

8. Simplified Modified Rankin Scale questionnaire (Circle each response on pathway \& final score)

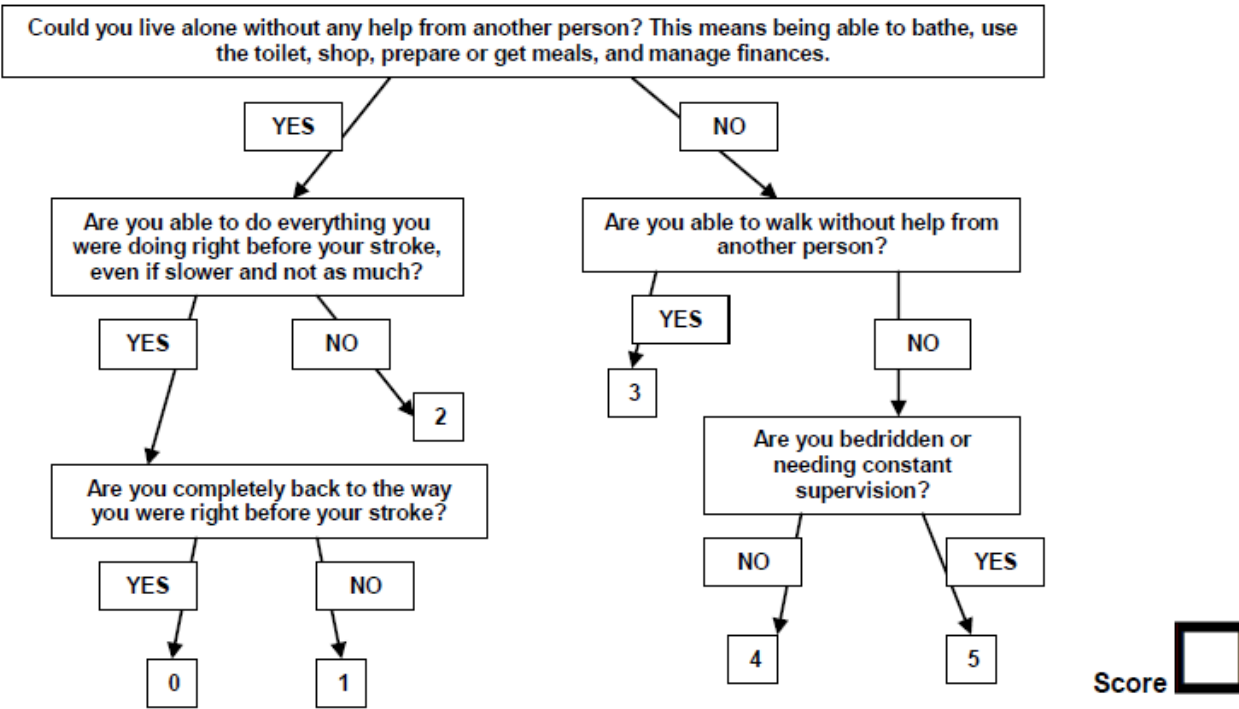

Secondary

\section{Outcomes}

\section{New clinical diagnosis of depression}

a. Has the patient been diagnosed with depression since the last assessment?

b. Has the patient been treated for depression since the last assessment? (non-pharmacological) Yes No $\square$

c. Has the patient been prescribed an antidepressant drug for treatment of depression since the last assessment?

If yes, to Q9c please ensure you have documented this on the current medication list. 
10. PHQ-9 (please circle) Over the past 2 weeks, have you been bothered by:

Scoring - $\quad 0$ : not at all; 1 : several days; 2 : more than half the days; 3 : nearly every day

\begin{tabular}{|c|l|c|c|c|c|}
\hline 1. & Little interest or pleasure in doing things? & 0 & 1 & 2 & 3 \\
\hline 2. & Feeling down, depressed, or hopeless? & 0 & 1 & 2 & 3 \\
\hline 3. & Trouble falling or staying asleep, or sleeping too much? & 0 & 1 & 2 & 3 \\
\hline 4. & Feeling tired or having little energy? & 0 & 1 & 2 & 3 \\
\hline 5. & Poor appetite or overeating? & 0 & 1 & 2 & 3 \\
\hline 6. & $\begin{array}{l}\text { Feeling bad about yourself- or that you are a failure or have let } \\
\text { yourself or your family down? }\end{array}$ & 0 & 1 & 2 & 3 \\
\hline 7. & $\begin{array}{l}\text { Trouble concentrating on things, such as reading the newspaper } \\
\text { or watching television? }\end{array}$ & 0 & 1 & 2 & 3 \\
\hline 8. & $\begin{array}{l}\text { Moving or speaking so slowly that other people could have } \\
\text { noticed. Or the opposite - being so fidgety or restless that you } \\
\text { have been moving around a lot more than usual? }\end{array}$ & 0 & 1 & 2 & 3 \\
\hline 9. & $\begin{array}{l}\text { Thoughts that you would be better off dead, or of hurting } \\
\text { yourself in some way? }\end{array}$ & 0 & 1 & 2 & 3 \\
\hline
\end{tabular}

TOTAL $\square$ If score $\geq 15 / 27$ notify GP: Yes $\square$ No $\square$ NA

Not able to complete

10. If you have checked off any problems, how difficult

Not difficult at all $\square$ have these problems made it for you to do your work, take care of things at home, or get along with other people?

Somewhat difficult $\square$

Very difficult $\square$

Extremely difficult $\square$

Please notify the participant that they will be followed-up centrally by the trial coordinating centre who will be in contact with them shortly via mail.

Please scan and email (affinitytrial@health.wa.gov.au or fax 086151 1028) the AFFINITY trial patient contact information sheet to the trial coordinating centre.

Please enter this information onto the corresponding form via the AFFINITY website www.affinitytrial.org

Please review/update the patients Hospital Admission and GP/Specialist diary and add any changes to the online form

End of 90 Day Assessment (thank you) 
Assessment Date: (dd/mm/yyy) $\square \square / \square \square / \square \square \square \square$ Collaborating centre:

\section{AFFINITY number $\square \square \square \square$ Patient Initials $\square \square$ Person completing this form:}

Main source of the data (please circle): Patient or Proxy or Medical Records or Other

1. Type of follow-up: (please circle)

Clinic Hospital Telephone Other (please list)

2. Survival: Is the patient alive at scheduled date of assessment?

Yes $\square$ No $\square$

(If deceased, please complete a serious adverse event form.)

3. Current Living Arrangements: Home alone $\square$ Living at home with someone else $\square$ Permanent care facility $\square$

$$
\text { Rehab } \square \quad \text { Other } \square \text { Please Specify }
$$

4. Current Medications: Please list all the medications the patient is currently taking

\begin{tabular}{|l|l|l|l|l|l|}
\hline \multicolumn{1}{|c|}{ Generic Name } & Start Date & Stop Date & \multicolumn{1}{|c|}{ Generic Name } & Start Date & Stop Date \\
\hline 1. & & & 8. & & \\
\hline 2. & & & 9. & & \\
\hline 3. & & & 10. & & \\
\hline 4. & & 11. & & \\
\hline 5. & & & 12. & & \\
\hline 6. & & & 13. & & \\
\hline 7. & & & 14. & & \\
\hline
\end{tabular}

\section{Medication Compliance}

a. On average, since last follow up how many times per week was the AFFINITY trial medication taken (patient report)?
(0) times per week
(1-2) times per week
(3-4) times per week
(5-6) times per week
(7) times per week

b. How many capsules are remaining in the bottle?

c. Did patient stop the trial medication at all since the last follow up?
Yes, temporarily
$\square$ If Yes, specify dates and reason;
Yes, permanently
$\square$ If Yes, specify dates and reason;
No
NA- permanently ceased reported on previous follow-up $\square$

Date Stopped $\square \square / \square \square / \square \square \square \square$

Date Re-started $\square \square / \square \square / \square \square \square \square$

Number of days off AFFINITY medication:

Reason: 


\section{End of AFFINITY Trial Medication}

Date completed AFFINITY trial medication (please enter the date of the last dose taken)

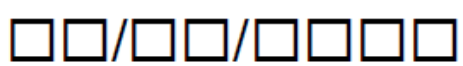

7. Serious Adverse Events and/or Secondary Outcome Events since 90 day follow-up: New stroke, ischaemic or haemorrhagic [not the qualifying stroke leading to enrolment] Yes Acute coronary syndrome [MI confirmed on ECG and/or raised serum Troponin] Yes Upper gastrointestinal bleed requiring blood transfusion and/or endoscopy Yes Other major bleed (i.e. not upper $\mathrm{Gl}$ or intracerebral)

- Requiring blood transfusion or procedural intervention Fall

New fracture [confirmed on X Ray]

Epileptic seizure [focal or generalised]

Symptomatic hypoglycaemia [blood sugar $<3 \mathrm{mmol} /$ ]

Symptomatic hyperglycaemia [blood sugar $>22 \mathrm{mmol} / \mathrm{l}]$

New hyponatraemia [ $\mathrm{Na}<125 \mathrm{mmol} / \mathrm{l}]$

Attempted suicide/self-harm

Other SAE

\begin{tabular}{llll} 
Y Yes & \multicolumn{3}{c}{ No $\square$} \\
Yes & $\square$ & No & $\square$ \\
Yes & $\square$ & No & $\square$ \\
Yes & $\square$ & No & $\square$ \\
& & & \\
Yes & $\square$ & No & $\square$ \\
Yes & $\square$ & No & $\square$ \\
Yes & $\square$ & No & $\square$ \\
Yes & $\square$ & No & $\square$ \\
Yes & $\square$ & No & $\square$ \\
Yes & $\square$ & No & $\square$ \\
Yes & $\square$ & No & $\square$ \\
Yes & $\square$ & No & $\square$ \\
Yes & $\square$ & No & $\square$
\end{tabular}

Please complete a "Serious Adverse Event and Secondary Outcome Event" Form immediately if a patient has answered 'Yes' to any of the above events that:

- are unexpected reactions to the AFFINITY trial medication (i.e. not consistent with the product information)

- result in death;

- are life threatening (i.e. the participant was at risk of death due to the event; it does not refer to an event which hypothetically might have caused death if it were more severe);

- require hospitalisation or prolongation of existing hospitalisation;

- result in persistent or significant disability or incapacity;

- is a congenital anomaly or birth defect;

- results in a secondary outcome event for the AFFINITY Trial (new stroke, ischaemic or haemorrhagic [not the qualifying stroke leading to enrolment], acute coronary syndrome [MI confirmed on ECG and/or raised serum Troponin], upper gastrointestinal bleed requiring blood transfusion and/or endoscopy, other major bleed (i.e not upper $\mathrm{Gl}$ or intracerebral) requiring blood transfusion or procedural intervention, new fracture [confirmed on X Ray], epileptic seizure [focal or generalised], new hyponatraemia $(\mathrm{Na}<125 \mathrm{mmol} / \mathrm{l})$ or attempted suicide/self-harm)

8. Pregnancy: Yes No

$\mathrm{N} / \mathrm{A}$

Please complete a separate 'pregnancy notification form'

9. Health care Utilisation:

a. Hospitalisation days since enrolment (not including hospitalisation for the initial/qualifying stroke)

b. Days spent in care at home since enrolment?

c. Formal carers - total number of visits per week? 


\section{Primary Outcome}

10. Simplified Modified Rankin Scale questionnaire (Circle each response on pathway \& final score)

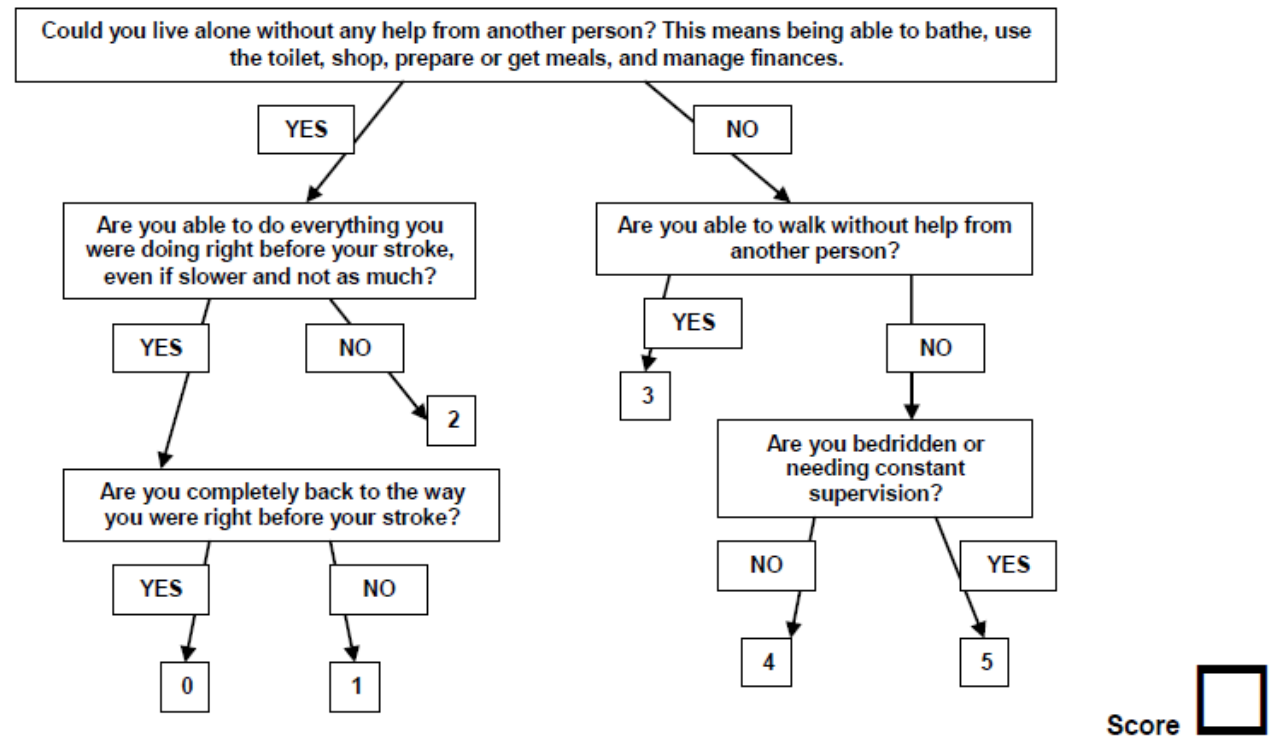




\section{Secondary Outcomes}

\section{New clinical diagnosis of depression}

a. Has the patient been diagnosed with depression since the last assessment?

b. Has the patient been treated for depression since the last assessment? (non-pharmacological) Yes $\square$ No $\square$

c. Has the patient been prescribed an antidepressant drug for treatment of depression since the last assessment?

If yes, to Q11c please ensure you have documented this on the current medication list.

12. PHQ-9 (please circle) Over the past 2 weeks, have you been bothered by:

Scoring - $\quad 0$ : not at all; 1 : several days; 2 : more than half the days; 3 : nearly every day

\begin{tabular}{|c|l|c|c|c|c|}
\hline 1. & Little interest or pleasure in doing things? & 0 & 1 & 2 & 3 \\
\hline 2. & Feeling down, depressed, or hopeless? & 0 & 1 & 2 & 3 \\
\hline 3. & Trouble falling or staying asleep, or sleeping too much? & 0 & 1 & 2 & 3 \\
\hline 4. & Feeling tired or having little energy? & 0 & 1 & 2 & 3 \\
\hline 5. & Poor appetite or overeating? & 0 & 1 & 2 & 3 \\
\hline 6. & $\begin{array}{l}\text { Feeling bad about yourself- or that you are a failure or have let } \\
\text { yourself or your family down? }\end{array}$ & 0 & 1 & 2 & 3 \\
\hline 7. & $\begin{array}{l}\text { Trouble concentrating on things, such as reading the newspaper } \\
\text { or watching television? }\end{array}$ & 0 & 1 & 2 & 3 \\
\hline 8. & $\begin{array}{l}\text { Moving or speaking so slowly that other people could have } \\
\text { noticed. Or the opposite - being so fidgety or restless that you } \\
\text { have been moving around a lot more than usual? }\end{array}$ & 0 & 1 & 2 & 3 \\
\hline 9. & $\begin{array}{l}\text { Thoughts that you would be better off dead, or of hurting } \\
\text { yourself in some way? }\end{array}$ & 0 & 1 & 2 & 3 \\
\hline
\end{tabular}

TOTAL $\square$ If score $\geq 15 / 27$ notify GP: Yes $\square$ No $\square$ NA $\square$ Not able to complete

10. If you checked off any problems, how difficult have these problems made it for you to do your work take care of things at home, or get along with other people?
Not difficult at all $\square$ Somewhat difficult $\square$ Very difficult $\square$ Extremely difficult $\square$ 
13. Cognition (TICSm)

Score ' 1 ' for each correct answer and ' 0 ' if incorrect in the boxes provided

\section{Orientation}

1. (i) What day of the week is it?

(ii) What is today's date?

(iii) What season are we in?

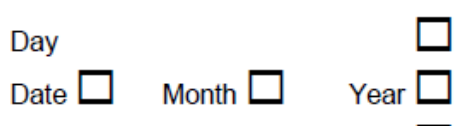

What is your age?

Season

3. What is your telephone number? (code $\&$ number)

Age

\section{Registration}

4. I'm going to read you a list of 10 words please listen carefully and try to remember them.

When I am done, tell me as many as you can in any order? Cabin $\square$ Pipe $\square$ Elephant $\square$ Chest $\square$ silk $\square$

Now tell me all the words you can remember. $\quad$ Theatre $\square$ Watch $\square$ Whip $\square$ Pillow $\square$ Giant $\square$

\section{Attention/Calculation}

5. Please take away 7 from 100

93

Now continue to take 7 away from what you have left over until I ask you to stop. 86

6

6. Please count backwards from 20 to 1

no mistakes

Comprehension, Semantic \& Recent Memory

7. What do people usually use to cut paper?

Scissors

8. What is the prickly green plant found in the desert

Cactus

9. Who is the reigning monarch now?

E, QE, QE2

10. Who is the Prime Minister now?

Correct surname

11. What is the opposite of east?

West

\section{Language/Repetition}

12. Please say this 'Methodist Episcopal'

Exactly right

\section{Delayed Recall}

13. Please repeat the list of 10 words I read earlier
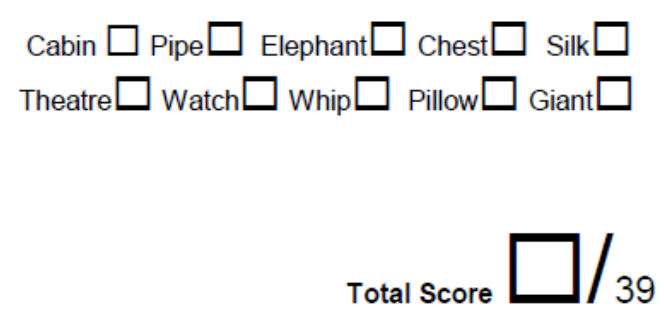
14. Fatigue (Vitality Domain of SF 36) (please circle)

The following questions are about how you feel and about how things have been with you in the past four weeks. As I read statement, please give me the one answer that comes closest to the way you have been feeling.

a. How much of the time during the past four weeks did you feel full of life?

\begin{tabular}{|c|c|c|c|c|c|}
\hline $\begin{array}{c}\text { All of the } \\
\text { time }\end{array}$ & $\begin{array}{c}\text { Most of the } \\
\text { time }\end{array}$ & $\begin{array}{c}\text { A good bit of the } \\
\text { time }\end{array}$ & $\begin{array}{c}\text { Some of the } \\
\text { time }\end{array}$ & $\begin{array}{c}\text { A little of the } \\
\text { time }\end{array}$ & $\begin{array}{c}\text { None of the } \\
\text { time }\end{array}$ \\
\hline
\end{tabular}

b. How much of the time during the past four weeks did you have a lot of energy?

\begin{tabular}{|c|c|c|c|c|c|}
\hline $\begin{array}{c}\text { All of the } \\
\text { time }\end{array}$ & $\begin{array}{c}\text { Most of the } \\
\text { time }\end{array}$ & $\begin{array}{c}\text { A good bit of the } \\
\text { time }\end{array}$ & $\begin{array}{c}\text { Some of the } \\
\text { time }\end{array}$ & $\begin{array}{c}\text { A little of the } \\
\text { time }\end{array}$ & $\begin{array}{c}\text { None of the } \\
\text { time }\end{array}$ \\
\hline
\end{tabular}

c. How much of the time during the past four weeks did you feel worn out?

\begin{tabular}{|c|c|c|c|c|c|}
\hline $\begin{array}{c}\text { All of the } \\
\text { time }\end{array}$ & $\begin{array}{c}\text { Most of the } \\
\text { time }\end{array}$ & $\begin{array}{c}\text { A good bit of the } \\
\text { time }\end{array}$ & $\begin{array}{c}\text { Some of the } \\
\text { time }\end{array}$ & $\begin{array}{c}\text { A little of the } \\
\text { time }\end{array}$ & $\begin{array}{c}\text { None of the } \\
\text { time }\end{array}$ \\
\hline
\end{tabular}

d. How much of the time during the past four weeks did you feel tired?

\begin{tabular}{|c|c|c|c|c|c|}
\hline $\begin{array}{c}\text { All of the } \\
\text { time }\end{array}$ & $\begin{array}{c}\text { Most of the } \\
\text { time }\end{array}$ & $\begin{array}{c}\text { A good bit of the } \\
\text { time }\end{array}$ & $\begin{array}{c}\text { Some of the } \\
\text { time }\end{array}$ & $\begin{array}{c}\text { A little of the } \\
\text { time }\end{array}$ & $\begin{array}{c}\text { None of the } \\
\text { time }\end{array}$ \\
\hline
\end{tabular}


15. Health-related quality of life (EQ-5D-5L):

Under each heading, please tick the ONE box that best describes your health TODAY Mobility

1. I have no problems with walking around

2. I have slight problems with walking around

3. I have moderate problems with walking around

4. I have severe problems with walking around

5. I am unable to walk around

6. I have no problems with washing or dressing myself $\quad \square$ 1

7. I have slight problems with washing or dressing myself $\quad \square 2$

8. I have moderate problems with washing or dressing myself $\square 3$

9. I have severe problems with washing or dressing myself $\quad \square 4$

10. I am unable to wash or dress myself $\quad \square 5$

Usual Activities (e.g. work, study, housework, family

or leisure activities)

11. I have no problems doing my usual activities $\quad \square$ 1

12. I have slight problems doing my usual activities $\quad \square 2$

13. I have moderate problems doing my usual activities $\quad \square 3$

14. I have severe problems doing my usual activities $\quad \square 4$

15. I am unable to do my usual activities $\quad \square 5$

Pain / Discomfort

16. I have no pain or discomfort $\quad \square$ 1

17. I have slight pain or discomfort $\quad \square 2$

18. I have moderate pain or discomfort $\quad \square 3$

19. I have severe pain or discomfort $\quad \square 4$

20. I have extreme pain or discomfort $\quad \square 5$

Anxiety / Depression

21. I am not anxious or depressed $\quad \square 1$

22. I am slightly anxious or depressed $\quad \square 2$

23. I am moderately anxious or depressed $\quad \square 3$

24. I am severely anxious or depressed $\quad \square 4$

25. I am extremely anxious or depressed $\quad \square 5$

- We would like to know how good or bad your health is TODAY,

- This scale is numbered from 0 to 100 .

- 100 means the best health you can imagine.

0 means the worst health you can imagine

- Mark an $\mathrm{X}$ on the scale on the next page to indicate how your health is TODAY.

- Now, please write the number you marked on the scale in the box. 
26. YOUR HEALTH TODAY =
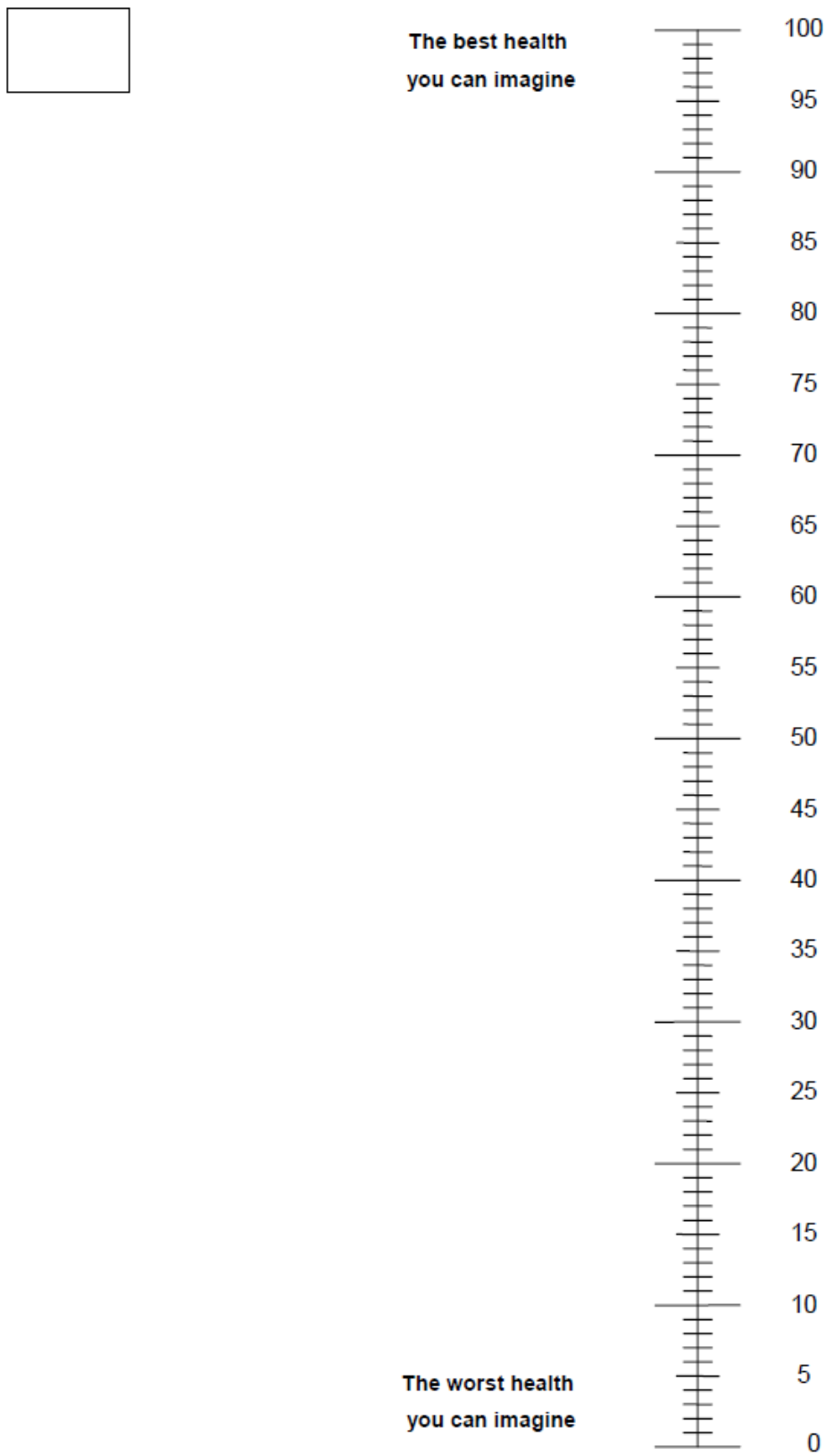
16. Overall health status (SIS) (please circle)

These questions are about the physical problems which may have occurred as a result of your stroke

\begin{tabular}{|l|c|c|c|c|c|}
\hline $\begin{array}{l}\text { 1. In the past week, how } \\
\text { would you rate the strength of } \\
\text { your... }\end{array}$ & $\begin{array}{c}\text { A lot of } \\
\text { strength }\end{array}$ & $\begin{array}{c}\text { Quite a bit of } \\
\text { strength }\end{array}$ & Some strength & A little strength & $\begin{array}{c}\text { No strength } \\
\text { at all }\end{array}$ \\
\hline $\begin{array}{l}\text { a. Arm that was most affected } \\
\text { by your stroke? }\end{array}$ & 5 & 4 & 3 & 2 & 1 \\
\hline $\begin{array}{l}\text { b. Grip of your hand that was } \\
\text { most affected by your stroke? }\end{array}$ & 5 & 4 & 3 & 2 & 1 \\
\hline $\begin{array}{l}\text { c. Leg that was most affected } \\
\text { by your stroke? }\end{array}$ & 5 & 4 & 3 & 2 & 1 \\
\hline $\begin{array}{l}\text { d. Foot/ankle that was most } \\
\text { affected by your stroke? }\end{array}$ & 5 & 4 & 3 & 2 & 1 \\
\hline
\end{tabular}

affected by your stroke?

Note: If you have no affected, or weaker, side then score your dominant side i.e. your right side if you are right handed, or your left side if you are left handed.

These questions are about your memory and thinking.

\begin{tabular}{|l|c|c|c|c|c|}
\hline $\begin{array}{l}\text { 2. In the past week, how } \\
\text { difficult was it for you to ... }\end{array}$ & $\begin{array}{c}\text { Not } \\
\text { difficult at } \\
\text { all }\end{array}$ & A little difficult & $\begin{array}{c}\text { Somewhat } \\
\text { difficult }\end{array}$ & Very difficult & $\begin{array}{c}\text { Extremely } \\
\text { difficult }\end{array}$ \\
\hline $\begin{array}{l}\text { a. Remember things that } \\
\text { people just told you? }\end{array}$ & 5 & 4 & 3 & 2 & 1 \\
\hline $\begin{array}{l}\text { b. Remember things that } \\
\text { happened the day before? }\end{array}$ & 5 & 4 & 3 & 2 & 1 \\
\hline $\begin{array}{l}\text { c. Remember to do things }(\text { e.g. } \\
\text { keep scheduled appointments } \\
\text { or take medication)? }\end{array}$ & 5 & 4 & 3 & 2 & 1 \\
\hline $\begin{array}{l}\text { d. Remember the day of the } \\
\text { week? }\end{array}$ & 5 & 4 & 3 & 2 & 1 \\
\hline e. Concentrate? & 5 & 4 & 3 & 2 & 1 \\
\hline f. Think quickly? & 5 & 4 & 3 & 2 & 1 \\
\hline g. Solve everyday problems? & 5 & 4 & 3 & & 1 \\
\hline
\end{tabular}

These questions are about how you feel, about changes in your mood and about your ability to control your emotions since your stroke.

\begin{tabular}{|l|c|c|c|c|c|}
\hline $\begin{array}{l}3 . \text { In the past week, how often } \\
\text { did you... }\end{array}$ & $\begin{array}{c}\text { None of the } \\
\text { time }\end{array}$ & $\begin{array}{c}\text { A little of the } \\
\text { time }\end{array}$ & $\begin{array}{c}\text { Some of the } \\
\text { time }\end{array}$ & Most of the time & $\begin{array}{c}\text { All of the } \\
\text { time }\end{array}$ \\
\hline a. Feel sad? & 5 & 4 & 3 & 2 & 1 \\
\hline $\begin{array}{l}\text { b. Feel that there is nobody } \\
\text { you are close to? }\end{array}$ & 5 & 4 & 3 & 2 & 1 \\
\hline $\begin{array}{l}\text { c. Feel that you are a burden } \\
\text { to others? }\end{array}$ & 5 & 4 & 3 & 2 & 1 \\
\hline $\begin{array}{l}\text { d. Feel that you have nothing } \\
\text { to look forward to? }\end{array}$ & 5 & 4 & 3 & 2 & 1 \\
\hline $\begin{array}{l}\text { e. Blame yourself for } \\
\text { mistakes that you made? }\end{array}$ & 5 & 4 & 3 & 2 & 1 \\
\hline $\begin{array}{l}\text { f. Enjoy things as much as } \\
\text { ever? }\end{array}$ & 5 & 4 & 3 & 2 & 1 \\
\hline $\begin{array}{l}\text { g. Feel quite nervous? } \\
\text { h. Feel that life is worth } \\
\text { living? }\end{array}$ & 5 & 4 & 3 & 2 & 1 \\
\hline $\begin{array}{l}\text { i. Smile and laugh at least } \\
\text { once a day? }\end{array}$ & 5 & 4 & 3 & 2 & 1 \\
\hline
\end{tabular}


The following questions are about your ability to communicate with other people, as well as your ability to understand what you read and what you hear in a conversation.

\begin{tabular}{|l|c|c|c|c|c|}
\hline $\begin{array}{l}\text { 4. In the past week, how } \\
\text { difficult was it to... }\end{array}$ & $\begin{array}{c}\text { Not difficult } \\
\text { at all }\end{array}$ & A little difficult & Somewhat difficult & Very difficult & $\begin{array}{c}\text { Extremely } \\
\text { difficult }\end{array}$ \\
\hline $\begin{array}{l}\text { a. Say the name of } \\
\text { someone in front of you? }\end{array}$ & 5 & 4 & 3 & 2 & 1 \\
\hline $\begin{array}{l}\text { b. Understand what was } \\
\text { being said to you in a } \\
\text { conversation? }\end{array}$ & 5 & 4 & 3 & 2 & 1 \\
\hline c. Reply to questions? & 5 & 4 & 3 & 2 & 1 \\
\hline $\begin{array}{l}\text { d. Correctly name } \\
\text { objects? }\end{array}$ & 5 & 4 & 3 & 2 & 1 \\
\hline $\begin{array}{l}\text { e. Participate in a } \\
\text { conversation with a } \\
\text { group of people? }\end{array}$ & 5 & 4 & 3 & 2 & 1 \\
\hline $\begin{array}{l}\text { f. Have a conversation } \\
\text { on the telephone? }\end{array}$ & 5 & 4 & 3 & & 1 \\
\hline $\begin{array}{l}\text { g. Call another person } \\
\text { on the telephone, } \\
\text { including selecting the } \\
\text { correct phone number } \\
\text { and dialling? }\end{array}$ & 5 & 4 & & & 2 \\
\hline
\end{tabular}

Notes: If you do not do any or all of these code them as Extremely Difficult.

Item $\mathrm{f}$. If you do not call but are handed the phone this is OK.

Item g. If you cannot hold a phone book, if you can read it this is OK. This item addresses whether you are able to initiate a phone call, look up the number, and dial this number correctly. 
The following questions ask about activities you might do during a typical day.

\begin{tabular}{|l|c|c|c|c|c|}
\hline $\begin{array}{l}5 . \text { In the past 2 weeks, } \\
\text { how difficult was it to... }\end{array}$ & $\begin{array}{c}\text { Not difficult } \\
\text { at all }\end{array}$ & A little difficult & Somewhat difficult & Very difficult & $\begin{array}{c}\text { Could not } \\
\text { do at all }\end{array}$ \\
\hline $\begin{array}{l}\text { a. Cut your food with a } \\
\text { knife and fork? }\end{array}$ & 5 & 4 & 3 & 2 & 1 \\
\hline $\begin{array}{l}\text { b. Dress the top part of } \\
\text { your body? }\end{array}$ & 5 & 4 & 3 & 2 & 1 \\
\hline c. Bathe yourself? & 5 & 4 & 3 & 2 & 1 \\
\hline d. Clip your toenails? & 5 & 4 & 3 & 2 & 1 \\
\hline $\begin{array}{l}\text { e. Get to the toilet on } \\
\text { time? }\end{array}$ & 5 & 4 & 3 & 2 & 1 \\
\hline $\begin{array}{l}\text { f. Control your bladder } \\
\text { (not have an accident)? }\end{array}$ & 5 & 4 & 3 & 2 & 1 \\
\hline $\begin{array}{l}\text { g. Control your bowels } \\
\text { (not have an accident)? }\end{array}$ & 5 & 4 & 3 & 2 & 1 \\
\hline $\begin{array}{l}\text { h. Do light household } \\
\text { tasks/chores (e.g. dust, } \\
\text { make a bed, take out the } \\
\text { garbage, do the dishes)? }\end{array}$ & 5 & 4 & & & 2 \\
\hline i. Go shopping? & 5 & 4 & 3 & 2 & 1 \\
\hline $\begin{array}{l}\text { j. Do heavy household } \\
\text { chores (vacuum, laundry } \\
\text { or yard work)? }\end{array}$ & 5 & 4 & & & 2 \\
\hline
\end{tabular}

Notes: If you do not do any or all of the activities listed, code them as Cannot do at all.

Item a. If you are on pureed food, even if you feel you could cut the food, code as Cannot do at all.

Item c. Bathing oneself does not include getting into the bath.

Item e. This question is associated with movement. Does the person have the physical ability to get to the bathroom quickly enough?

Item f. Losing a little urine/dribbling is considered an accident. If you have a catheter, code as Cannot do at all. Item g. Constipation is not counted here, person has to have an accident.

Item i. "Shopping" means any type of shopping and does not include driving.

The following questions are about your mobility at home and in the community.

\begin{tabular}{|l|c|c|c|c|c|}
\hline $\begin{array}{l}\text { 6. In the past 2 weeks, } \\
\text { how difficult was it to.. }\end{array}$ & $\begin{array}{c}\text { Not difficult } \\
\text { at all }\end{array}$ & A little difficult & Somewhat difficult & Very difficult & $\begin{array}{c}\text { Could not } \\
\text { do at all }\end{array}$ \\
\hline $\begin{array}{l}\text { a. Stay sitting without } \\
\text { losing your balance? }\end{array}$ & 5 & 4 & 3 & 2 & 1 \\
\hline $\begin{array}{l}\text { b. Stay standing without } \\
\text { losing your balance? }\end{array}$ & 5 & 4 & 3 & 2 & 1 \\
\hline $\begin{array}{l}\text { c. Walk without losing } \\
\text { your balance? }\end{array}$ & 5 & 4 & 3 & 2 & 1 \\
\hline $\begin{array}{l}\text { d. Move from a bed to a } \\
\text { chair? }\end{array}$ & 5 & 4 & 3 & 2 & 1 \\
\hline e. Walk one block? & 5 & 4 & 3 & 2 & 1 \\
\hline f. Walk fast? & 5 & 4 & 3 & 2 & 1 \\
\hline $\begin{array}{l}\text { g. Climb one flight of } \\
\text { stairs? }\end{array}$ & 5 & 4 & 3 & 2 & 1 \\
\hline $\begin{array}{l}\text { h. Climb several flights } \\
\text { of stairs? }\end{array}$ & 5 & 4 & 3 & 2 & 1 \\
\hline $\begin{array}{l}\text { i. Get in and out of the } \\
\text { car? }\end{array}$ & 5 & 4 & & & 2 \\
\hline
\end{tabular}

Notes: If you have not done any of the items in the past two weeks code as Cannot do at all. 
The following questions are about your ability to use your hand that was MOST AFFECTED by your stroke.

\begin{tabular}{|l|c|c|c|c|c|}
\hline $\begin{array}{l}\text { 7. In the past 2 weeks, } \\
\text { how difficult was it to use } \\
\text { your hand that was most } \\
\text { affected by your stroke } \\
\text { to... }\end{array}$ & $\begin{array}{c}\text { Not difficult } \\
\text { at all }\end{array}$ & A little difficult & Somewhat difficult & Very difficult & $\begin{array}{c}\text { Could not } \\
\text { do at all }\end{array}$ \\
\hline $\begin{array}{l}\text { a. Carry heavy objects } \\
\text { (e.g. bag of groceries)? }\end{array}$ & 5 & 4 & 3 & 2 & 1 \\
\hline b. Turn a doorknob? & 5 & 4 & 3 & 2 & 1 \\
\hline c. Open a can or jar? & 5 & 4 & 3 & 2 & 1 \\
\hline d. Tie a shoe lace? & 5 & 4 & 3 & 2 & 1 \\
\hline e. Pick up a 5 cent piece? & 5 & 4 & 3 & 2 & 1 \\
\hline
\end{tabular}

Notes: If you have no affected, or weaker, side then score your dominant side i.e. your right side if you are right handed, or your left side if you are left handed.

The following questions are about how stroke has affected your ability to participate in the activities that you usually do, things that are meaningful to you and help you to find purpose in life

\begin{tabular}{|c|c|c|c|c|c|}
\hline $\begin{array}{l}\text { 8. In the past } 4 \text { weeks, } \\
\text { how much of the time have } \\
\text { you been limited in... }\end{array}$ & $\begin{array}{l}\text { None of the } \\
\text { time }\end{array}$ & $\begin{array}{l}\text { A little of the } \\
\text { time }\end{array}$ & Some of the time & Most of the time & $\begin{array}{l}\text { All of the } \\
\text { time }\end{array}$ \\
\hline $\begin{array}{l}\text { a. Your work (paid, } \\
\text { voluntary or other)? }\end{array}$ & 5 & 4 & 3 & 2 & 1 \\
\hline b. Your social activities? & 5 & 4 & 3 & 2 & 1 \\
\hline $\begin{array}{l}\text { c. Quiet recreation (crafts, } \\
\text { reading)? }\end{array}$ & 5 & 4 & 3 & 2 & 1 \\
\hline $\begin{array}{l}\text { d. Active recreation } \\
\text { (sports, outings, travel)? }\end{array}$ & 5 & 4 & 3 & 2 & 1 \\
\hline $\begin{array}{l}\text { e. Your role as a family } \\
\text { member and/or friend? }\end{array}$ & 5 & 4 & 3 & 2 & 1 \\
\hline $\begin{array}{l}\text { f. Your participation in } \\
\text { spiritual or religious } \\
\text { activities? }\end{array}$ & 5 & 4 & 3 & 2 & 1 \\
\hline $\begin{array}{l}\text { g. Your ability to control } \\
\text { your life as you wish? }\end{array}$ & 5 & 4 & 3 & 2 & 1 \\
\hline $\begin{array}{l}\text { h. Your ability to help } \\
\text { others? }\end{array}$ & 5 & 4 & 3 & 2 & 1 \\
\hline
\end{tabular}

Notes: If you don't do any of the specific items, and has never done, code as None of the time. 
9. On a scale of 0 to 100 , with 100 representing full recovery and 0 representing no recovery, how much have you recovered from your stroke?

-100 Full Recovery
-
-90
-
-80
-
-70
-
-60
-
-50
-
-40
-
-30
-
-20
-
-10
-
-0 No recovery

Please notify the participant that we will be in contact with them 6 months from now (i.e. 12 months from date of Randomisation)

Please enter this information onto the corresponding form via the AFFINITY website www.affinitytrial.org

End of 180 Day Assessment (thank you) 
Assessment Date: (dd/mm/yyy) $\square \square / \square \square / \square \square \square \square$ Collaborating centre:

\section{AFFINITY number $\square \square \square \square$ Patient Initials $\square \square$ Person completing this form:}

Main source of the data (please circle): Patient or Proxy or Medical Records or Other

1. Type of follow-up: (please circle)

Clinic Hospital Telephone Other (please list)

2. Survival: Is the patient alive at scheduled date of assessment?

Yes $\square$ No $\square$

(If deceased, please complete a serious adverse event form.)

3. Current Living Arrangements: Home alone $\square$ Living at home with someone else $\square$ Permanent care facility

Rehab

Other $\square$ Please Specify

4. Current Medications: Please list all the medications the patient is currently taking

\begin{tabular}{|l|l|l|l|l|l|}
\hline \multicolumn{1}{|c|}{ Generic Name } & Start Date & Stop Date & \multicolumn{1}{|c|}{ Generic Name } & Start Date & Stop Date \\
\hline 1. & & & 8. & & \\
\hline 2. & & & 9. & & \\
\hline 3. & & & 10. & & \\
\hline 4. & & & 11. & & \\
\hline 5. & & & 12. & & \\
\hline 6. & & & 13. & & \\
\hline 7. & & & 14. & & \\
\hline
\end{tabular}

5. Serious Adverse Events and/or Secondary Outcome Events since 180 day follow-up: New stroke, ischaemic or haemorrhagic [not the qualifying stroke leading to enrolment] Yes Acute coronary syndrome [MI confirmed on ECG and/or raised serum Troponin] Yes Upper gastrointestinal bleed requiring blood transfusion and/or endoscopy Yes Other major bleed (i.e. not upper GI or intracerebral)

- Requiring blood transfusion or procedural intervention Fall

New fracture [confirmed on X Ray]

Epileptic seizure [focal or generalised]

Symptomatic hypoglycaemia [blood sugar $<3 \mathrm{mmol} /$ ]

Symptomatic hyperglycaemia [blood sugar $>22 \mathrm{mmol} / \mathrm{l}]$

New hyponatraemia $[\mathrm{Na}<125 \mathrm{mmol} / \mathrm{l}]$

Attempted suicide/self-harm

Other SAE

\begin{tabular}{ccc}
\multicolumn{3}{c}{ Yes $\square$} \\
$\square$ & No \\
$\square$ & No & $\square$ \\
$\square$ & No & $\square$ \\
& & \\
$\square$ & No & $\square$ \\
$\square$ & No & $\square$ \\
$\square$ & No & $\square$ \\
$\square$ & No & $\square$ \\
$\square$ & No & $\square$ \\
$\square$ & No & $\square$ \\
$\square$ & No & $\square$ \\
$\square$ & No & $\square$ \\
$\square$ & No & $\square$
\end{tabular}

Please complete a "Serious Adverse Event and Secondary Outcome Event" Form immediately if a patient has answered 'Yes' to any of the above events that: 
- are unexpected reactions to the AFFINITY trial medication (i.e. not consistent with the product information)

- result in death;

- are life threatening (i.e. the participant was at risk of death due to the event, it does not refer to an event which hypothetically might have caused death if it were more severe);

- require hospitalisation or prolongation of existing hospitalisation;

- result in persistent or significant disability or incapacity;

- is a congenital anomaly or birth defect;

- results in a secondary outcome event for the AFFINITY Trial (new stroke, ischaemic or haemorrhagic [not the qualifying stroke leading to enrolment], acute coronary syndrome [MI confirmed on ECG and/or raised serum Troponin], upper gastrointestinal bleed requiring blood transfusion and/or endoscopy, other major bleed (i.e. not upper $\mathrm{Gl}$ or intracerebral) requiring blood transfusion or procedural intervention, new fracture [confirmed on X Ray], epileptic seizure [focal or generalised], new hyponatraemia [ $\mathrm{Na}<125 \mathrm{mmol} / \mathrm{l}]$ or attempted suicide/self-harm).

\section{Pregnancy: Yes $\square$ No $\square$ N/A}

Please complete a separate 'pregnancy notification form'.

\section{Health care Utilisation:}

a. Hospitalisation days since enrolment (not including hospitalisation for the initial/qualifying stroke)

b. Days spent in care at home since enrolment?

c. Formal carers - total number of visits per week?

\section{Primary Outcome}

8. Simplified Modified Rankin Scale questionnaire (Circle each response on pathway \& final score)

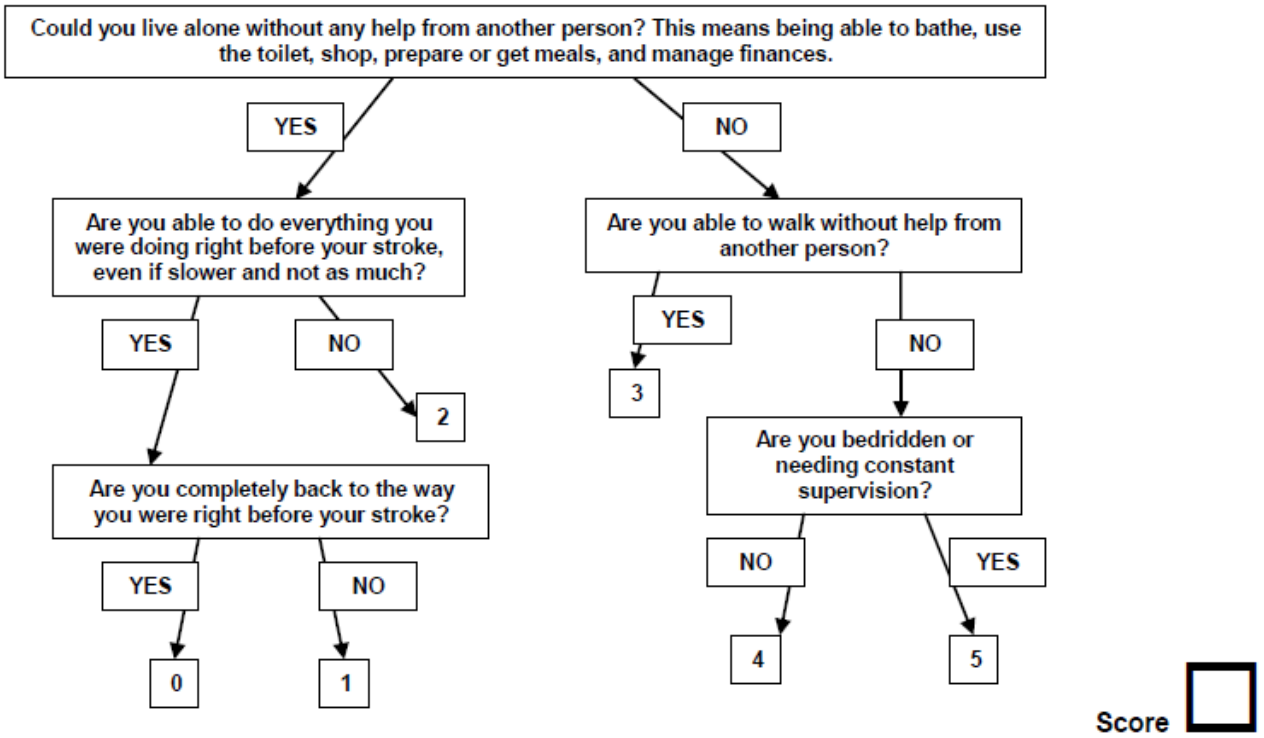




\section{Secondary Outcomes}

9. New clinical diagnosis of depression

a. Has the patient been diagnosed with depression since the last assessment?

b. Has the patient been treated for depression since the last assessment? (non-pharmacological)

Yes $\square$ No $\square$

c. Has the patient been prescribed an antidepressant drug for treatment of depression since the last assessment?

If yes, to Q9c please ensure you have documented this on the current medication list.

Yes $\square$ No $\square$

10. PHQ-9 (please circle) Over the past 2 weeks, have you been bothered by:

Scoring - $\quad 0$ : not at all; 1 : several days; 2 : more than half the days; 3 : nearly every day

\begin{tabular}{|c|l|c|c|c|c|}
\hline 1. & Little interest or pleasure in doing things? & 0 & 1 & 2 & 3 \\
\hline 2. & Feeling down, depressed, or hopeless? & 0 & 1 & 2 & 3 \\
\hline 3. & Trouble falling or staying asleep, or sleeping too much? & 0 & 1 & 2 & 3 \\
\hline 4. & Feeling tired or having little energy? & 0 & 1 & 2 & 3 \\
\hline 5. & Poor appetite or overeating? & 0 & 1 & 2 & 3 \\
\hline 6. & $\begin{array}{l}\text { Feeling bad about yourself- or that you are a failure or have let } \\
\text { yourself or your family down? }\end{array}$ & 0 & 1 & 2 & 3 \\
\hline 7. & $\begin{array}{l}\text { Trouble concentrating on things, such as reading the newspaper } \\
\text { or watching television? }\end{array}$ & 0 & 1 & 2 & 3 \\
\hline 8. & $\begin{array}{l}\text { Moving or speaking so slowly that other people could have } \\
\text { noticed. Or the opposite - being so fidgety or restless that you } \\
\text { have been moving around a lot more than usual? }\end{array}$ & 0 & 1 & 2 & 3 \\
\hline 9. & $\begin{array}{l}\text { Thoughts that you would be better off dead, or of hurting } \\
\text { yourself in some way? }\end{array}$ & 0 & 1 & 2 & 3 \\
\hline
\end{tabular}

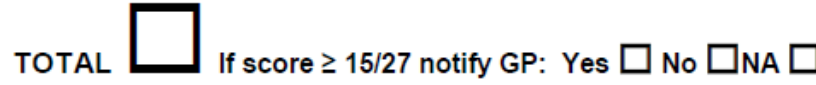

Not able to complete $\square$

10. If you checked off any problems, how difficult have these problems made it for you to do your work, take care of things at home, or get along with other people?
Not difficult at all $\square$ Somewhat difficult $\square$ Very difficult $\square$

Extremely difficult $\square$ 
11. Cognition (TICSm)

Score ' 1 ' for each correct answer and ' 0 ' if incorrect in the boxes provided

\section{Orientation}

1. (i) What day of the week is it?

(ii) What is today's date?

(iii) What season are we in?

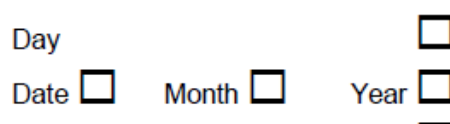

What is your age?

3. What is your telephone number? (code \& number)

Season

\section{Registration}

4. I'm going to read you a list of 10 words please listen carefully and try to remember them.

When I am done, tell me as many as you can in any order? Cabin $\square$ Pipe $\square$ Elephant $\square$ Chest $\square$ silk $\square$

Now tell me all the words you can remember. $\quad$ Theatre $\square$ Watch $\square$ Whip $\square$ Pillow $\square$ Giant $\square$

\section{Attention/Calculation}

5. Please take away 7 from 100

$93 \square$

Now continue to take 7 away from what you have left over until I ask you to stop. 86

6. Please count backwards from 20 to 1

no mistakes

\section{Comprehension, Semantic \& Recent Memory}

7. What do people usually use to cut paper?

8. What is the prickly green plant found in the desert

Scissors

9. Who is the reigning monarch now?

Cactus

10. Who is the Prime Minister now?

E, QE, QE2

11. What is the opposite of east?

Correct surname

Language/Repetition

12. Please say this 'Methodist Episcopal'

West

\section{Delayed Recall}

13. Please repeat the list of 10 words I read earlier

Cabin $\square$ Pipe $\square$ Elephant $\square$ Chest $\square$ silk $\square$
Theatre $\square$ Watch $\square$ Whip $\square$ Pillow $\square$ Giant $\square$

Total Score $\square / 39$ 
12. Fatigue (Vitality Domain of SF 36) (please circle)

The following questions are about how you feel and about how things have been with you in the past four weeks. As I read statement, please give me the one answer that comes closest to the way you have been feeling.

a. How much of the time during the past four weeks did you feel full of life?

\begin{tabular}{|c|c|c|c|c|c|}
\hline $\begin{array}{c}\text { All of the } \\
\text { time }\end{array}$ & $\begin{array}{c}\text { Most of the } \\
\text { time }\end{array}$ & $\begin{array}{c}\text { A good bit of the } \\
\text { time }\end{array}$ & $\begin{array}{c}\text { Some of the } \\
\text { time }\end{array}$ & $\begin{array}{c}\text { A little of the } \\
\text { time }\end{array}$ & $\begin{array}{c}\text { None of the } \\
\text { time }\end{array}$ \\
\hline
\end{tabular}

b. How much of the time during the past four weeks did you have a lot of energy?

\begin{tabular}{|c|c|c|c|c|c|}
\hline $\begin{array}{c}\text { All of the } \\
\text { time }\end{array}$ & $\begin{array}{c}\text { Most of the } \\
\text { time }\end{array}$ & $\begin{array}{c}\text { A good bit of the } \\
\text { time }\end{array}$ & $\begin{array}{c}\text { Some of the } \\
\text { time }\end{array}$ & $\begin{array}{c}\text { A little of the } \\
\text { time }\end{array}$ & $\begin{array}{c}\text { None of the } \\
\text { time }\end{array}$ \\
\hline c. How much of the time during the past four weeks did you feel worn out? \\
\hline $\begin{array}{c}\text { All of the } \\
\text { time }\end{array}$ & $\begin{array}{c}\text { Most of the } \\
\text { time }\end{array}$ & $\begin{array}{c}\text { A good bit of the } \\
\text { time }\end{array}$ & $\begin{array}{c}\text { Some of the } \\
\text { time }\end{array}$ & $\begin{array}{c}\text { A little of the } \\
\text { time }\end{array}$ & $\begin{array}{c}\text { None of the } \\
\text { time }\end{array}$ \\
\hline \begin{tabular}{c} 
d. How much of the time during the past four weeks did you feel tired? \\
\hline $\begin{array}{c}\text { All of the } \\
\text { time }\end{array}$
\end{tabular}$\quad \begin{array}{c}\text { Most of the } \\
\text { time }\end{array}$ & $\begin{array}{c}\text { A good bit of the } \\
\text { time }\end{array}$ & $\begin{array}{c}\text { Some of the } \\
\text { time }\end{array}$ & $\begin{array}{c}\text { A little of the } \\
\text { time }\end{array}$ & $\begin{array}{c}\text { None of the } \\
\text { time }\end{array}$ \\
\hline
\end{tabular}


13. Health-related quality of life (EQ-5D-5L):

Under each heading, please tick the ONE box that best describes your health TODAY

Mobility

1. I have no problems with walking around $\quad \square 1$

2. I have slight problems with walking around $\quad \square 2$

3. I have moderate problems with walking around $\quad \square 3$

4. I have severe problems with walking around $\quad \square_{4}$

5. I am unable to walk around $\quad \square 5$

Personal Care

6. I have no problems with washing or dressing myself $\quad \square$ 1

7. I have slight problems with washing or dressing myself $\quad \square 2$

8. I have moderate problems with washing or dressing myself $\square 3$

9. I have severe problems with washing or dressing myself $\quad \square_{4}$

10. I am unable to wash or dress myself $\quad \square 5$

Usual Activities (e.g. work, study, housework, family

or leisure activities)

11. I have no problems doing my usual activities $\quad \square$ 1

12. I have slight problems doing my usual activities $\quad \square 2$

13. I have moderate problems doing my usual activities $\quad \square 3$

14. I have severe problems doing my usual activities $\quad \square 4$

15. I am unable to do my usual activities $\quad \square 5$

Pain / Discomfort

16. I have no pain or discomfort $\quad \square 1$

17. I have slight pain or discomfort $\quad \square 2$

18. I have moderate pain or discomfort $\quad \square 3$

19. I have severe pain or discomfort $\quad \square 4$

20. I have extreme pain or discomfort $\quad \square 5$

Anxiety / Depression

21. I am not anxious or depressed $\quad \square 1$

22. I am slightly anxious or depressed $\quad \square 2$

23. I am moderately anxious or depressed $\quad \square 3$

24. I am severely anxious or depressed $\quad \square 4$

25. I am extremely anxious or depressed $\quad \square 5$

- We would like to know how good or bad your health is TODAY.

- This scale is numbered from 0 to 100 .

- 100 means the best health you can imagine. 0 means the worst health you can imagine

- Mark an $\mathrm{X}$ on the scale on the next page to indicate how your health is TODAY.

- Now, please write the number you marked on the scale in the box.

AFFINITY 365 Day Assessment Form Version $6,18^{\text {th }}$ November 2015

Page 6 of 12 
26. YOUR HEALTH TODAY =

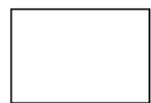

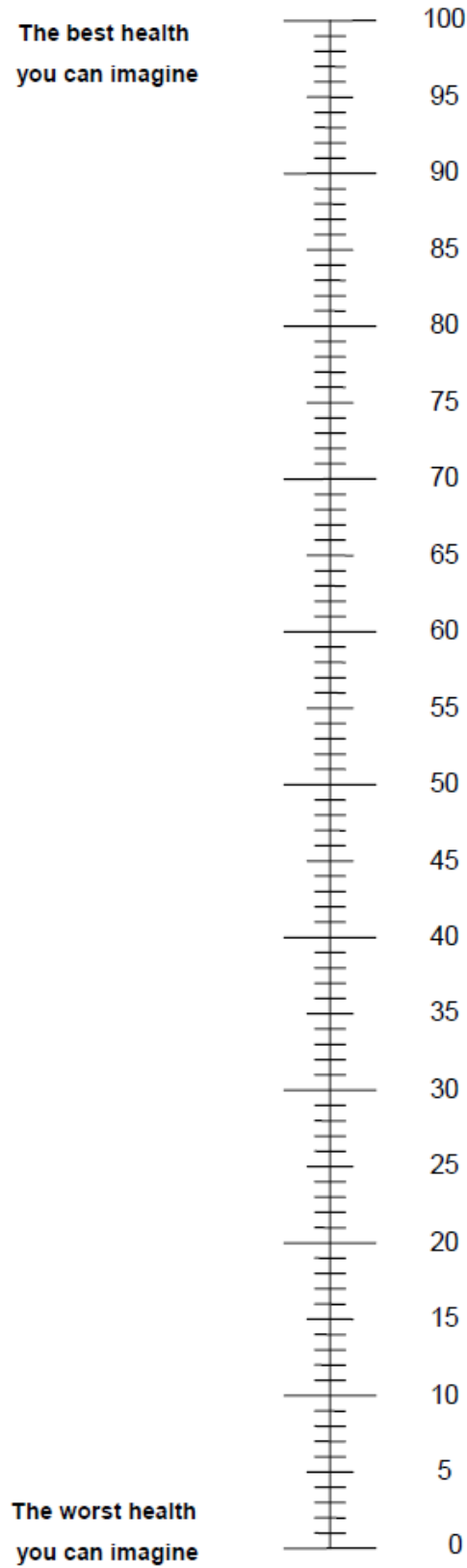

you can imagine

Page 7 of 12 
14. Overall health status (SIS) (please circle)

These questions are about the physical problems which may have occurred as a result of your stroke

\begin{tabular}{|l|c|c|c|c|c|}
\hline $\begin{array}{l}\text { 1. In the past week, how } \\
\text { would you rate the strength of } \\
\text { your... }\end{array}$ & $\begin{array}{c}\text { A lot of } \\
\text { strength }\end{array}$ & $\begin{array}{c}\text { Quite a bit of } \\
\text { strength }\end{array}$ & Some strength & A little strength & $\begin{array}{c}\text { No strength } \\
\text { at all }\end{array}$ \\
\hline $\begin{array}{l}\text { a. Arm that was most affected } \\
\text { by your stroke? }\end{array}$ & 5 & 4 & 3 & 2 & 1 \\
\hline $\begin{array}{l}\text { b. Grip of your hand that was } \\
\text { most affected by your stroke? }\end{array}$ & 5 & 4 & 3 & 2 & 1 \\
\hline $\begin{array}{l}\text { c. Leg that was most affected } \\
\text { by your stroke? }\end{array}$ & 5 & 4 & 3 & 2 & 1 \\
\hline $\begin{array}{l}\text { d. Foot/ankle that was most } \\
\text { affected by your stroke? }\end{array}$ & 5 & 4 & 3 & 2 & 1 \\
\hline
\end{tabular}

Note: If you have no affected, or weaker, side then score your dominant side i.e. your right side if you are right handed, or your left side if you are left handed.

These questions are about your memory and thinking.

\begin{tabular}{|l|c|c|c|c|c|}
\hline $\begin{array}{l}\text { 2. In the past week, how } \\
\text { difficult was it for you to ... }\end{array}$ & $\begin{array}{c}\text { Not } \\
\text { difficult at } \\
\text { all }\end{array}$ & A little difficult & $\begin{array}{c}\text { Somewhat } \\
\text { difficult }\end{array}$ & Very difficult & $\begin{array}{c}\text { Extremely } \\
\text { difficult }\end{array}$ \\
\hline $\begin{array}{l}\text { a. Remember things that } \\
\text { people just told you? }\end{array}$ & 5 & 4 & 3 & 2 & 1 \\
\hline $\begin{array}{l}\text { b. Remember things that } \\
\text { happened the day before? }\end{array}$ & 5 & 4 & 3 & 2 & 1 \\
\hline $\begin{array}{l}\text { c. Remember to do things }(\text { e.g. } \\
\text { keep scheduled appointments } \\
\text { or take medication)? }\end{array}$ & 5 & 4 & 3 & 2 & 1 \\
\hline $\begin{array}{l}\text { d. Remember the day of the } \\
\text { week? }\end{array}$ & 5 & 4 & 3 & 2 & 1 \\
\hline e. Concentrate? & 5 & 4 & 3 & 2 & 1 \\
\hline f. Think quickly? & 5 & 4 & 3 & 2 & 1 \\
\hline g. Solve everyday problems? & 5 & 4 & 3 & & 1 \\
\hline
\end{tabular}

These questions are about how you feel, about changes in your mood and about your ability to control your emotions since your stroke.

\begin{tabular}{|l|c|c|c|c|c|}
\hline $\begin{array}{l}3 . \text { In the past week, how often } \\
\text { did you... }\end{array}$ & $\begin{array}{c}\text { None of the } \\
\text { time }\end{array}$ & $\begin{array}{c}\text { A little of the } \\
\text { time }\end{array}$ & $\begin{array}{c}\text { Some of the } \\
\text { time }\end{array}$ & Most of the time & $\begin{array}{c}\text { All of the } \\
\text { time }\end{array}$ \\
\hline a. Feel sad? & 5 & 4 & 3 & 2 & 1 \\
\hline $\begin{array}{l}\text { b. Feel that there is nobody } \\
\text { you are close to? }\end{array}$ & 5 & 4 & 3 & 2 & 1 \\
\hline $\begin{array}{l}\text { c. Feel that you are a burden } \\
\text { to others? }\end{array}$ & 5 & 4 & 3 & 2 & 1 \\
\hline $\begin{array}{l}\text { d. Feel that you have nothing } \\
\text { to look forward to? }\end{array}$ & 5 & 4 & 3 & 2 & 1 \\
\hline $\begin{array}{l}\text { e. Blame yourself for } \\
\text { mistakes that you made? }\end{array}$ & 5 & 4 & 3 & 2 & 1 \\
\hline $\begin{array}{l}\text { f. Enjoy things as much as } \\
\text { ever? }\end{array}$ & 5 & 4 & 3 & 2 & 1 \\
\hline $\begin{array}{l}\text { g. Feel quite nervous? } \\
\text { h. Feel that life is worth } \\
\text { living? }\end{array}$ & 5 & 4 & 3 & 2 & 1 \\
\hline $\begin{array}{l}\text { i. Smile and laugh at least } \\
\text { once a day? }\end{array}$ & 5 & 4 & 3 & 2 & 1 \\
\hline
\end{tabular}


The following questions are about your ability to communicate with other people, as well as your ability to understand what you read and what you hear in a conversation.

\begin{tabular}{|c|c|c|c|c|c|}
\hline $\begin{array}{l}\text { 4. In the past week, how } \\
\text { difficult was it to... }\end{array}$ & $\begin{array}{l}\text { Not difficult } \\
\text { at all }\end{array}$ & A little difficult & Somewhat difficult & Very difficult & $\begin{array}{l}\text { Extremely } \\
\text { difficult }\end{array}$ \\
\hline $\begin{array}{l}\text { a. Say the name of } \\
\text { someone in front of you? }\end{array}$ & 5 & 4 & 3 & 2 & 1 \\
\hline $\begin{array}{l}\text { b. Understand what was } \\
\text { being said to you in a } \\
\text { conversation? }\end{array}$ & 5 & 4 & 3 & 2 & 1 \\
\hline C. Reply to questions? & 5 & 4 & 3 & 2 & 1 \\
\hline $\begin{array}{l}\text { d. Correctly name } \\
\text { objects? }\end{array}$ & 5 & 4 & 3 & 2 & 1 \\
\hline $\begin{array}{l}\text { e. Participate in a } \\
\text { conversation with a } \\
\text { group of people? }\end{array}$ & 5 & 4 & 3 & 2 & 1 \\
\hline $\begin{array}{l}\text { f. Have a conversation } \\
\text { on the telephone? }\end{array}$ & 5 & 4 & 3 & 2 & 1 \\
\hline $\begin{array}{l}\text { g. Call another person } \\
\text { on the telephone, } \\
\text { including selecting the } \\
\text { correct phone number } \\
\text { and dialling? }\end{array}$ & 5 & 4 & 3 & 2 & 1 \\
\hline
\end{tabular}

Notes: If you do not do any or all of these code them as Extremely Difficult.

Item $\mathrm{f}$. If you do not call but are handed the phone this is OK.

Item g. If you cannot hold a phone book, if you can read it this is OK. This item addresses whether you are able to initiate a phone call, look up the number, and dial this number correctly. 
The following questions ask about activities you might do during a typical day.

\begin{tabular}{|l|c|c|c|c|c|}
\hline $\begin{array}{l}5 . \text { In the past 2 weeks, } \\
\text { how difficult was it to... }\end{array}$ & $\begin{array}{c}\text { Not difficult } \\
\text { at all }\end{array}$ & A little difficult & Somewhat difficult & Very difficult & $\begin{array}{c}\text { Could not } \\
\text { do at all }\end{array}$ \\
\hline $\begin{array}{l}\text { a. Cut your food with a } \\
\text { knife and fork? }\end{array}$ & 5 & 4 & 3 & 2 & 1 \\
\hline $\begin{array}{l}\text { b. Dress the top part of } \\
\text { your body? }\end{array}$ & 5 & 4 & 3 & 2 & 1 \\
\hline c. Bathe yourself? & 5 & 4 & 3 & 2 & 1 \\
\hline d. Clip your toenails? & 5 & 4 & 3 & 2 & 1 \\
\hline $\begin{array}{l}\text { e. Get to the toilet on } \\
\text { time? }\end{array}$ & 5 & 4 & 3 & 2 & 1 \\
\hline $\begin{array}{l}\text { f. Control your bladder } \\
\text { (not have an accident)? }\end{array}$ & 5 & 4 & 3 & 2 & 1 \\
\hline $\begin{array}{l}\text { g. Control your bowels } \\
\text { (not have an accident)? }\end{array}$ & 5 & 4 & 3 & 2 & 1 \\
\hline $\begin{array}{l}\text { h. Do light household } \\
\text { tasks/chores (e.g. dust, } \\
\text { make a bed, take out the } \\
\text { garbage, do the dishes)? }\end{array}$ & 5 & 4 & & & 2 \\
\hline $\begin{array}{l}\text { i. Go shopping? } \\
\text { j. Do heavy household } \\
\text { chores (vacuum, laundry } \\
\text { or yard work)? }\end{array}$ & 5 & & 3 & & 2 \\
\hline
\end{tabular}

Notes: If you do not do any or all of the activities listed, code them as Cannot do at all.

Item a. If you are on pureed food, even if you feel you could cut the food, code as Cannot do at all.

Item c. Bathing oneself does not include getting into the bath.

Item e. This question is associated with movement. Does the person have the physical ability to get to the bathroom quickly enough?

Item f. Losing a little urine/dribbling is considered an accident. If you have a catheter, code as Cannot do at all. Item g. Constipation is not counted here, person has to have an accident.

Item i. "Shopping" means any type of shopping and does not include driving.

The following questions are about your mobility at home and in the community.

\begin{tabular}{|l|c|c|c|c|c|}
\hline $\begin{array}{l}\text { 6. In the past 2 weeks, } \\
\text { how difficult was it to... }\end{array}$ & $\begin{array}{c}\text { Not difficult } \\
\text { at all }\end{array}$ & A little difficult & Somewhat difficult & Very difficult & $\begin{array}{c}\text { Could not } \\
\text { do at all }\end{array}$ \\
\hline $\begin{array}{l}\text { a. Stay sitting without } \\
\text { losing your balance? }\end{array}$ & 5 & 4 & 3 & 2 & 1 \\
\hline $\begin{array}{l}\text { b. Stay standing without } \\
\text { losing your balance? }\end{array}$ & 5 & 4 & 3 & 2 & 1 \\
\hline $\begin{array}{l}\text { c. Walk without losing } \\
\text { your balance? }\end{array}$ & 5 & 4 & 3 & 2 & 1 \\
\hline $\begin{array}{l}\text { d. Move from a bed to a } \\
\text { chair? }\end{array}$ & 5 & 4 & 3 & 2 & 1 \\
\hline e. Walk one block? & 5 & 4 & 3 & 2 & 1 \\
\hline f. Walk fast? & 5 & 4 & 3 & 2 & 1 \\
\hline $\begin{array}{l}\text { g. Climb one flight of } \\
\text { stairs? }\end{array}$ & 5 & 4 & 3 & 2 & 1 \\
\hline $\begin{array}{l}\text { h. Climb several flights } \\
\text { of stairs? }\end{array}$ & 5 & 4 & 3 & 2 & 1 \\
\hline $\begin{array}{l}\text { i. Get in and out of the } \\
\text { car? }\end{array}$ & 5 & 4 & & & 2 \\
\hline
\end{tabular}

Notes: If you have not done any of the items in the past two weeks code as Cannot do at all. 
The following questions are about your ability to use your hand that was MOST AFFECTED by your stroke.

\begin{tabular}{|l|c|c|c|c|c|}
\hline $\begin{array}{l}\text { 7. In the past 2 weeks, } \\
\text { how difficult was it to use } \\
\text { your hand that was most } \\
\text { affected by your stroke } \\
\text { to... }\end{array}$ & $\begin{array}{c}\text { Not difficult } \\
\text { at all }\end{array}$ & A little difficult & Somewhat difficult & Very difficult & $\begin{array}{c}\text { Could not } \\
\text { do at all }\end{array}$ \\
\hline $\begin{array}{l}\text { a. Carry heavy objects } \\
\text { (e.g. bag of groceries)? }\end{array}$ & 5 & 4 & 3 & 2 & 1 \\
\hline b. Turn a doorknob? & 5 & 4 & 3 & 2 & 1 \\
\hline c. Open a can or jar? & 5 & 4 & 3 & 2 & 1 \\
\hline d. Tie a shoe lace? & 5 & 4 & 3 & 2 & 1 \\
\hline e. Pick up a 5 cent piece? & 5 & 4 & 3 & 2 & 1 \\
\hline
\end{tabular}

Notes: If you have no affected, or weaker, side then score your dominant side i.e. your right side if you are right handed, or your left side if you are left handed.

The following questions are about how stroke has affected your ability to participate in the activities that you usually do, things that are meaningful to you and help you to find purpose in life

\begin{tabular}{|c|c|c|c|c|c|}
\hline $\begin{array}{l}\text { 8. In the past } 4 \text { weeks, } \\
\text { how much of the time have } \\
\text { you been limited in... }\end{array}$ & $\begin{array}{l}\text { None of the } \\
\text { time }\end{array}$ & $\begin{array}{l}\text { A little of the } \\
\text { time }\end{array}$ & Some of the time & Most of the time & $\begin{array}{l}\text { All of the } \\
\text { time }\end{array}$ \\
\hline $\begin{array}{l}\text { a. Your work (paid, } \\
\text { voluntary or other)? }\end{array}$ & 5 & 4 & 3 & 2 & 1 \\
\hline b. Your social activities? & 5 & 4 & 3 & 2 & 1 \\
\hline $\begin{array}{l}\text { c. Quiet recreation (crafts, } \\
\text { reading)? }\end{array}$ & 5 & 4 & 3 & 2 & 1 \\
\hline $\begin{array}{l}\text { d. Active recreation } \\
\text { (sports, outings, travel)? }\end{array}$ & 5 & 4 & 3 & 2 & 1 \\
\hline $\begin{array}{l}\text { e. Your role as a family } \\
\text { member and/or friend? }\end{array}$ & 5 & 4 & 3 & 2 & 1 \\
\hline $\begin{array}{l}\text { f. Your participation in } \\
\text { spiritual or religious } \\
\text { activities? }\end{array}$ & 5 & 4 & 3 & 2 & 1 \\
\hline $\begin{array}{l}\text { g. Your ability to control } \\
\text { your life as you wish? }\end{array}$ & 5 & 4 & 3 & 2 & 1 \\
\hline $\begin{array}{l}\text { h. Your ability to help } \\
\text { others? }\end{array}$ & 5 & 4 & 3 & 2 & 1 \\
\hline
\end{tabular}

Notes: If you don't do any of the specific items, and has never done, code as None of the time. 
9. On a scale of 0 to 100 , with 100 representing full recovery and 0 representing no recovery, how much have you recovered from your stroke?

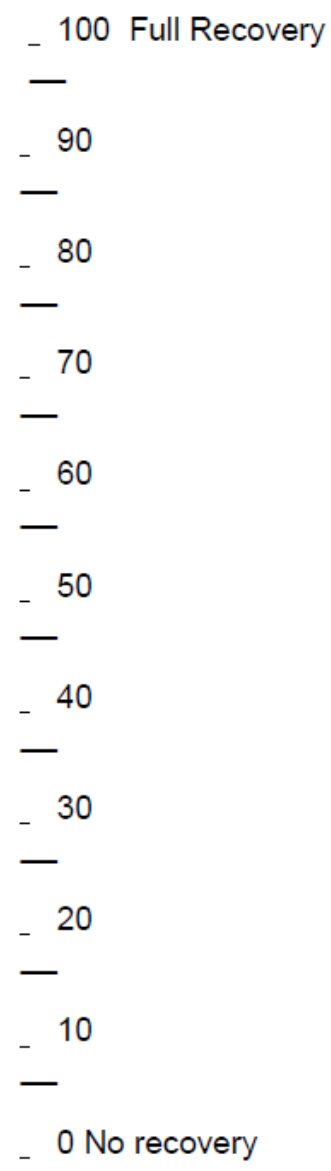

Please inform the patient (and carer, if appropriate) that their participation in the AFFINITY trial is now completed and thank them for their contribution.

Please enter this information onto the corresponding form via the AFFINITY website www.affinitytrial.org

End of 365 Day Assessment (thank you) 